UNIVERSIDADE DE SÃO PAULO

FACULDADE DE SAÚDE PÚBLICA

\title{
Variações da fauna de mosquitos (Diptera: Culicidae) em área de implantação de uma hidrelétrica no rio Paranapanema, SP/PR.
}

\section{Renato Sinnhofer Sugimoto}

Dissertação apresentada ao Programa de PósGraduação da Faculdade de Saúde Pública da Universidade de São Paulo, para a obtenção do título de Mestre em Saúde Pública.

Área de concentração: Epidemiologia

Orientador: Prof. Delsio Natal

SÃO PAULO

2009 


\section{Variações da fauna de mosquitos (Diptera: Culicidae) em área de implantação de uma hidrelétrica no rio Paranapanema, SP/PR.}

Renato Sinnhofer Sugimoto

Dissertação apresentada ao Programa de PósGraduação da Faculdade de Saúde Pública da Universidade de São Paulo, para a obtenção do título de Mestre em Saúde Pública.

Área de concentração: Epidemiologia

Orientador: Prof. Delsio Natal

SÃO PAULO

2009 
É expressamente proibida a comercialização deste documento, tanto na sua forma impressa como eletrônica. Sua reprodução total ou parcial é permitida exclusivamente para fins acadêmicos e científicos, desde que na reprodução figure a identificação do autor, título, instituição e ano da dissertação 
Aos meus pais Massao e Monica, força e razão de tudo que sou.

A Vanessa, síntese do apoio e amor recebidos.

Aos meus avôs Hans (in memorian), Therezinha, Yoshihiko (in memorian) e Haruko, exemplos de vida. 


\section{Agradecimentos}

À minha família por todo o apoio, educação e exemplo de vida, pois sem vocês não seria possivel trilhar meu caminho até aqui.

À Vanessa dos Santos Marques, pela companhia e paciência, durante todo o trajeto, e também pela revisão e sugestões na escrita desta dissertação.

Ao Prof. Delsio Natal pela orientação, amizade, apoio e incentivo permanente na realização deste trabalho e minha carreira. E pela realização das coletas de campo.

Ao Prof. Titular Almério de Castro Gomes, pela realização das coletas.

Ao Paulo Roberto Urbinatti, biólogo do Laboratório de Entomologia em Saúde Pública da FSP-USP, pela realização das coletas, diagnóstico do material coletado e pelo apoio em todas as etapas deste trabalho.

À Dra. Regiane Maria Tironi de Menezes, bióloga da SUCEN, São Paulo, pelas correções e explicações da parte de índices estatísticos.

Aos biólogos Aristides Fernandes e Márcia Bicudo de Paula, do Laboratório de Entomologia em Saúde Pública da FSP-USP, pelo diagnóstico do material coletado e pela paciência ao me ensinar preparação e diagnose de formas imaturas e machos de mosquito.

Ao Walter Ceretti Junior, biólogo do Laboratório de Entomologia em Saúde Pública da FSP-USP, pelas conversas e discussões sobre os mais diversos assuntos.

Aos meus colegas do mestrado e FUNDAP pelos momentos de descontração.

À Companhia Brasileira de Alumínio, por ter concedido a utilização dos dados de monitoramento para a realização deste trabalho.

Ao Cnpq, pela concessão da bolsa de mestrado que viabilizou a realização desta dissertação.

A todos aqueles que, direta ou indiretamente, ajudaram na realização deste estudo. 
...O homem chega e já desfaz a natureza Tira a gente põe represa, diz que tudo vai mudar

O São Francisco lá prá cima da Bahia Diz que dia menos dia vai subir bem devagar E passo a passo vai cumprindo a profecia Do beato que dizia que o sertão ia alagar...

...Adeus remanso, casa nova, sento-sé Adeus pilão arcado vem o rio te engolir Debaixo d'água lá se vai a vida inteira Por cima da cachoeira o gaiola vai subir Vai ter barragem no salto do Sobradinho E o povo vai se embora com medo de se afogar...

Sobradinho Sá \& Guarabyra 


\section{Resumo}

Se por um lado as usinas hidrelétricas suprem as necessidades de energia do país, essas também são responsáveis pela proliferação de doenças cujos agentes são transmitidos, em sua grande maioria, por mosquitos que utilizam as áreas inundadas dessas usinas como criadouros. Pode-se observar tal cenário na UHE de Ourinhos, que está localizada no rio Paranapanema entre as cidades de Ourinhos, SP, e Jacarezinho, PR, onde foram definidas três estações de coleta usadas para a avaliação da diversidade das populações de mosquitos nos períodos pré e pós inundação do reservatório, a partir dos dados coletados descreveu-se as alterações ocorridas na população de culicídeos da UHE Ourinhos, diante dos impactos da inundação e calculou-se riqueza, dominância, diversidade $\alpha$ e $\beta$, IAEP e similaridade. Sendo que para captura de adultos utilizou-se armadilha de Shannon e aspirador à bateria, e para as formas imaturas "conchas entomológicas” em coleções de água estagnada. Entre as espécies com importância epidemiológica coletadas e identificadas temos: Aedes albopictus, Aedes scapularis, Anopheles albitarsis s.l, Anopheles darlingi, Culex quinquefasciatus, Culex nigripalpus, Haemagogus leucocelaenus e Psorophora ferox. Destaca-se a redução do número de espécies no período pós enchimento, juntamente com a recolonização do nicho por novas espécies ou espécies antes raras, como exemplificado pelo surgimento do An. darlingi, e ascensão do An. albitarsis s.l, espécies consideradas vetores primário e secundário da malária, no Brasil, respectivamente. Como conclusão, depreende-se que o lago formado pela UHE Ourinhos alterou a diversidade de culicídeos local, fato que pode representar menor contato desses hematófagos com a população no entorno do lago recém formado.

Palavras-chave: ecologia, mosquitos, vetores, hidrelétrica, Ourinhos 


\begin{abstract}
If the hydroelectric power plants supply the needs of the country, they are also responsible for the proliferation of diseases whose pathogens are transferred, in most of time, by mosquitoes that using the flooded areas such as breeding. It can be observed this scenario in the HPP of Ourinhos, which is located on the Paranapanema river between the cities of Ourinhos, SP, and Jacarezinho, PR, where three stations were established to collect used for assessing the diversity of populations in the pre and post flooding of the reservoir from the data collected described the changes occurring in the population of Culicidae of Ourinhos HPP, before the impacts of flooding and it was estimated richness, dominance, $\alpha$ and $\beta$ diversity, standardized index of abundance of species and similarity. Where to catch the adult was used the Shannon's trap and vacuum, and for the immature forms "entomological ladle" in collections of stagnant water. Among the species of epidemiological importance have collected and identified: Aedes albopictus, Aedes scapularis, Anopheles albitarsis s.l, Anopheles darlingi, Culex quinquefasciatus, Culex nigripalpus, Haemagogus leucocelaenus and Psorophora ferox. It is reducing the number of species in the period after filling, together with the recolonization of the niche for new species or rare species before, as exemplified by the emergence of Anopheles(Nys.) darlingi and Anopheles albitarsis s.l rise of species, as primary and secondary vectors malaria in Brazil, respectively. In conclusion, it appears that the lake formed by the dam of Ourinhos changed the diversity of the mosquitoes in site, which may represent less contact with the blood of people around the newly formed lake.
\end{abstract}

Keywords: ecology, mosquitoes, vectors, hydroelectric, Ourinhos 


\section{Sumário}

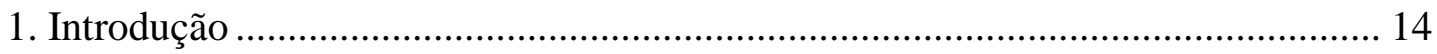

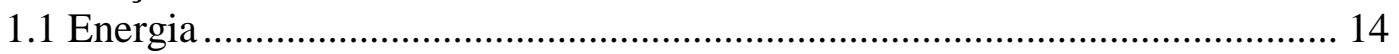

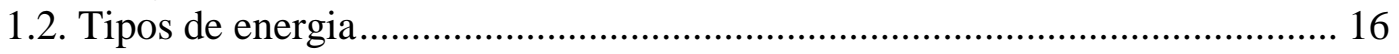

1.3. O modelo energético brasileiro e seus impactos.......................................... 17

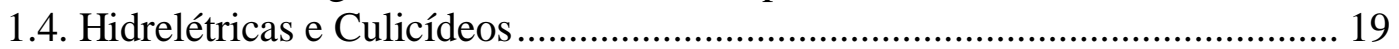

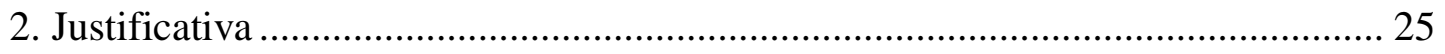

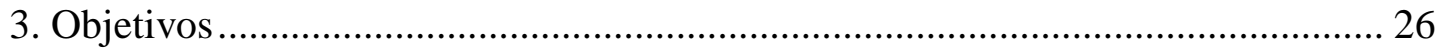

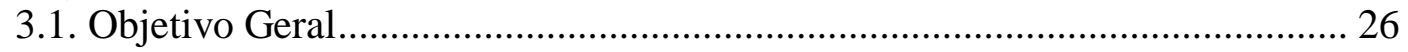

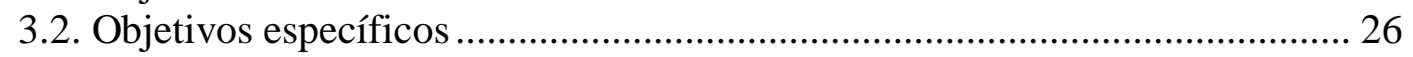

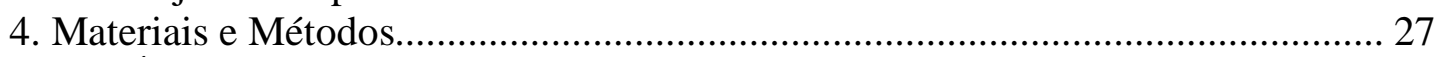

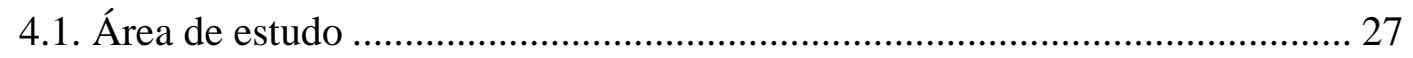

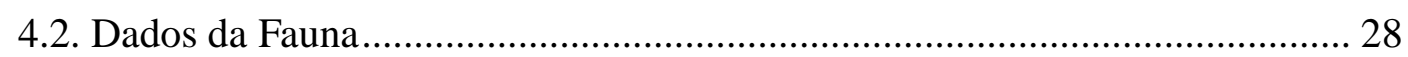

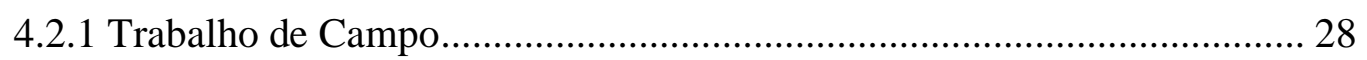

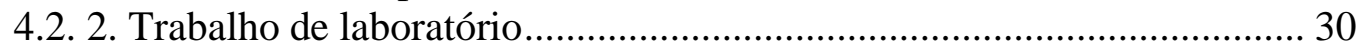

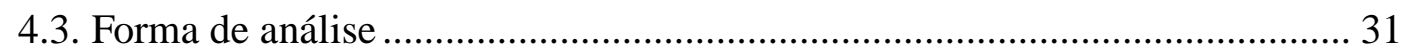

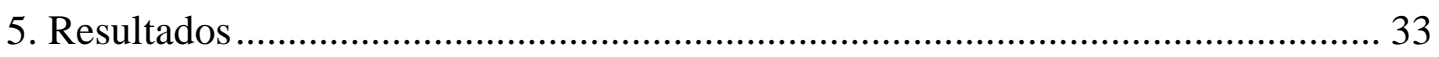

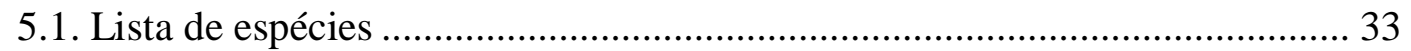

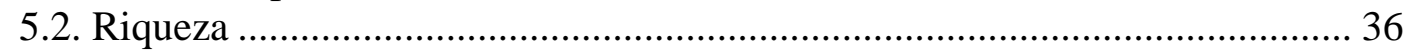

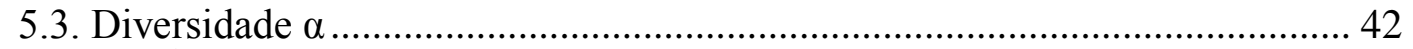

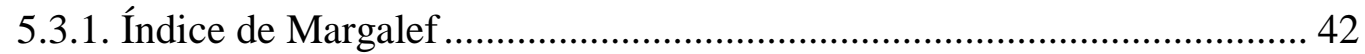

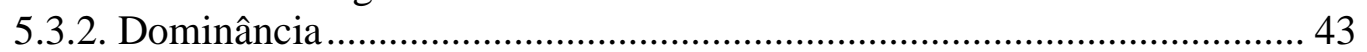

5.3.3. Índice de Abundância de Espécie Padronizado ....................................... 44

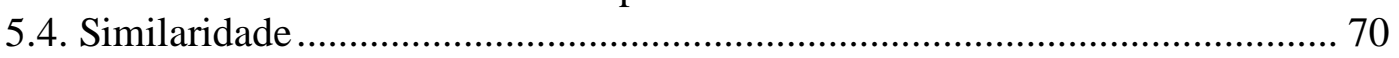

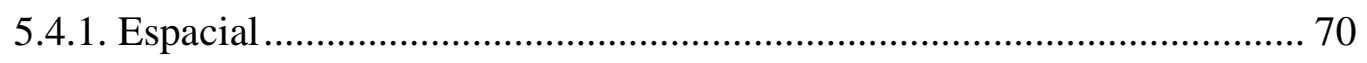

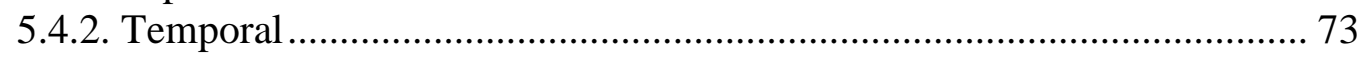

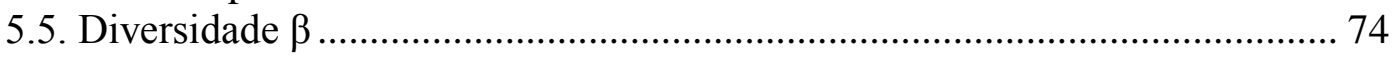

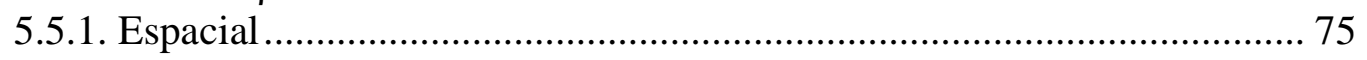

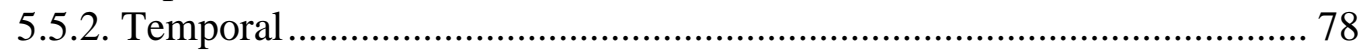

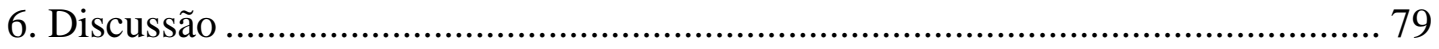

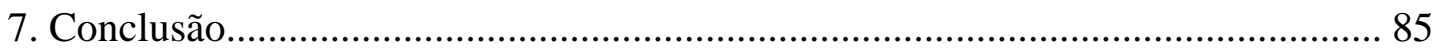

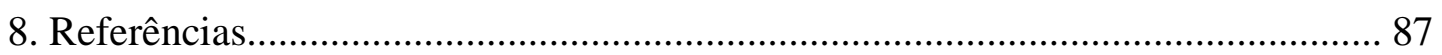

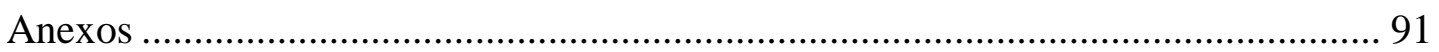

Anexo I - Área de estudo e estações de coleta de mosquitos com indicações das

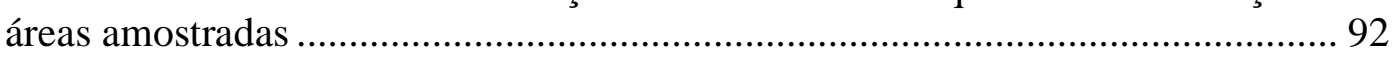

Anexo II - Paisagens das estações de coleta de mosquitos ................................... 95

Anexo III - Técnicas de coleta de mosquitos ........................................................... 97

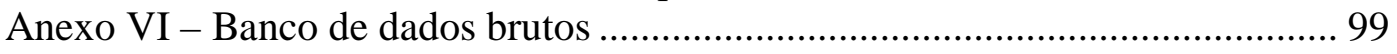

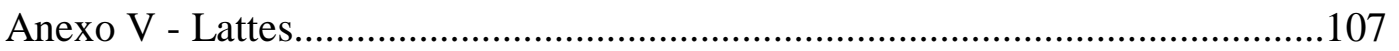




\section{Índice de Figuras, Quadros e Tabelas}

Figura 1. Riqueza segundo estações de coleta e campanhas obtidas nas coletas com concha entomológica para avaliação de formas imaturas, no monitoramento da Usina Hidrelétrica de Ourinhos.

Figura 2. Riqueza segundo estações de coleta e campanhas obtidas nas coletas com aspirador elétrico, no monitoramento da Usina Hidrelétrica de Ourinhos.

Figura 3. Riqueza segundo estações de coleta e campanhas obtidas nas coletas com armadilha de Shannon, no monitoramento da Usina Hidrelétrica de Ourinhos.

Figura 4. Riqueza segundo estações de coleta e campanhas obtidas no monitoramento da Usina Hidrelétrica de Ourinhos.

Figura 5. Curva de rarefação, segundo número de indivíduos coletados, para a estação de coleta Canteiro de Obras, no monitoramento da Usina Hidrelétrica de Ourinhos, para os períodos Pré e Pós enchimento.

Figura 6. Curva de rarefação, segundo número de indivíduos coletados, para a estação de coleta Sítio Caiçara, no monitoramento da Usina Hidrelétrica de Ourinhos, para os períodos Pré e Pós enchimento.

Figura 7. Curva de rarefação, segundo número de indivíduos coletados, para a estação de coleta Sítio Caiçara, no monitoramento da Usina Hidrelétrica de Ourinhos, para os períodos Pré e Pós enchimento.

Figura 8. Índice de Magalef $(D)$ dos períodos pré e pós enchimento, segundo método e estação de coleta, e geral.

Figura 9. Dominância (Indíce de Simpson) dos períodos Pré e Pós enchimento, segundo método e estação de coleta, e geral. . .44 Figura 10. IAEP na estação de coleta Canteiro de Obras obtida nas coletas com concha entomológica para avaliação de formas imaturas, no monitoramento da Usina Hidrelétrica de Ourinhos, nos períodos pré (a) e pós (b) enchimento do reservatório.

Figura 11. IAEP na estação de coleta Sítio Caiçara obtida nas coletas com concha entomológica para avaliação de formas imaturas, no monitoramento da Usina Hidrelétrica de Ourinhos, nos períodos pré (a) e pós (b) enchimento do reservatório

Figura 12. IAEP na estação de coleta Córrego Anhumas obtida nas coletas com concha entomológica para avaliação de formas imaturas, no monitoramento da Usina Hidrelétrica de Ourinhos, nos períodos pré (a) e pós (b) enchimento do reservatório

Figura 13. IAEP na estação de coleta Canteiro de Obras obtida nas coletas com aspirador elétrico, no monitoramento da Usina Hidrelétrica de Ourinhos, nos períodos pré (a) e pós (b) enchimento do reservatório.

Figura 14. IAEP na estação de coleta Sítio Caiçara obtida nas coletas com aspirador elétrico, no monitoramento da Usina Hidrelétrica de Ourinhos, nos períodos pré (a) e pós (b) enchimento do reservatório.

Figura 15. IAEP na estação de coleta Córrego Anhumas obtida nas coletas com aspirador elétrico, no monitoramento da Usina Hidrelétrica de Ourinhos, nos períodos pré (a) e pós (b) enchimento do reservatório.

Figura 16. IAEP na estação de coleta Canteiro de Obras obtida nas coletas com armadilha de Shannon, no monitoramento da Usina Hidrelétrica de Ourinhos, nos períodos pré (a) e pós (b) enchimento do reservatório.

Figura 17. IAEP na estação de coleta Sítio Caiçara obtida nas coletas com armadilha de Shannon, no monitoramento da Usina Hidrelétrica de Ourinhos, nos períodos pré (a) e pós (b) enchimento do reservatório.

Figura 18. IAEP na estação de coleta Córrego Anhumas obtida nas coletas com armadilha de Shannon, no monitoramento da Usina Hidrelétrica de Ourinhos, nos períodos pré (a) e pós (b) enchimento do reservatório. 
Figura 19. IAEP na estação de coleta Canteiro de Obras obtida pelas três técnicas de coleta, no monitoramento da Usina Hidrelétrica de Ourinhos, nos períodos pré (a) e pós (b) enchimento do reservatório.

Figura 20. IAEP na estação de coleta Sítio Caiçara obtida pelas três técnicas de coleta, no monitoramento da Usina Hidrelétrica de Ourinhos, nos períodos pré (a) e pós (b) enchimento do reservatório.

Figura 21. IAEP na estação de coleta Córrego Anhumas obtida pelas três técnicas de coleta, no monitoramento da Usina Hidrelétrica de Ourinhos, nos períodos pré (a) e pós (b) enchimento do reservatório.

Figura 22. Coeficiente de Similaridade de Jaccard entre estações de coleta, obtida nas coletas com concha entomológica para avaliação de formas imaturas, no monitoramento da Usina Hidrelétrica de Ourinhos, nos períodos pré e pós enchimento do reservatório.

Figura 23. Coeficiente de Similaridade de Jaccard entre estações de coleta, obtida nas coletas com aspirador elétrico, no monitoramento da Usina Hidrelétrica de Ourinhos, nos períodos pré e pós enchimento do reservatório. ....

Figura 24. Coeficiente de Similaridade de Jaccard entre estações de coleta, obtida nas coletas com armadilha de Shannon, no monitoramento da Usina Hidrelétrica de Ourinhos, nos períodos pré e pós enchimento do reservatório.

Figura 25. Coeficiente de Similaridade de Jaccard entre estações de coleta, no monitoramento da Usina Hidrelétrica de Ourinhos, nos períodos pré e pós enchimento do reservatório. ........................73 Figura 26. Coeficiente de Similaridade Temporal entre os períodos pré e pós enchimento do reservatório segundo as estações de coleta e técnica de coleta, do monitoramento da Usina Hidrelétrica de Ourinhos, SP. Brasil.

Figura 27. Diversidade 6 entre estações de coleta, obtida nas coletas com concha entomológica para avaliação de formas imaturas, nos períodos pré e pós enchimento do reservatório da Usina Hidrelétrica de Ourinhos........................................................................................................75 Figura 28. Diversidade 6 entre estações de coleta, obtida nas coletas com aspirador elétrico, na Usina Hidrelétrica de Ourinhos, nos períodos pré e pós enchimento do reservatório...................................76 Figura 29. Diversidade $B$ entre estações de coleta, obtida nas coletas com armadilha de Shannon, na Usina Hidrelétrica de Ourinhos, nos períodos pré e pós enchimento do reservatório. ....................... 77 Figura 30. Diversidade $b$ entre estações de coleta, obtida utilizando-se as três técnicas de coleta, na Usina Hidrelétrica de Ourinhos, nos períodos pré e pós enchimento do reservatório. ........................77 Figura 31. Diversidade 6 Temporal entre os períodos pré e pós enchimento do reservatório segundo as estações de coleta e técnica de coleta, do monitoramento da Usina Hidrelétrica de Ourinhos. ..... 78 Figura 32. Hidrelétrica Ourinhos - Vista Aérea ...........................................................................92 Figura 33. Hidrelétrica Ourinhos - Vista por satélite ...............................................................92 Figura 34. Localização das estações de coleta - Vista por satélite. Círculo - Canteiro de obras; Triângulo - Sítio Caiçara; Losângulo - Córrego Anhumas ...........................................................93 Figura 35. Estação de coleta de culicídeos Canteiro de Obras - localizada na margem direita do Rio Paranapanema, no município de Ourinhos, nas proximidades do eixo da barragem, à jusante. São mostradas as áreas onde foram feitas as coletas de mosquitos adultos com armadilha de Shannon, com aspirador elétrico e coletas de formas imaturas com concha entomológica. Figura 36. Estação de coleta de culicídeos Sítio Caiçara - localizada na margem direita do Rio Paranapanema, no município de Chavantes, SP. São mostradas as áreas onde foram feitas as coletas de mosquitos com armadilha de Shannon, com aspirador elétrico e com concha entomológica........94 Figura 37. Estação de coleta de culicídeos Córrego Anhumas - localizada na margem esquerda do Rio Paranapanema, no município de Ribeirão Claro, PR. São mostradas as áreas onde foram feitas as coletas com armadilha de Shannon, com aspirador elétrico e concha entomológica.

Figura 38. Estação de coleta de culicídeos Canteiro de Obras - vista a partir das instalações do canteiro. Em primeiro plano observa-se o domínio da taboa (Typha sp.). Ao fundo, à esquerda destaca-se a mata onde foi instalada a armadilha de Shannon em sua borda. 
Figura 39. Aspecto do lago recém-formado na região da estação de coleta Sítio Caiçara. Em primeiro plano observa-se área de pastagem, no segundo a área inundada.. No canto esquerdo superior observa-se, em ponto mais elevado, a sede do sítio utilizado para coleta..... 95 Figura 40. Estação de coleta de culicídeos Córrego Anhumas - vista a partir da margem esquerda do rio Paranapanema. Em primeiro plano observa-se área de extração de argila. Em segundo plano é mostrada a mata residual utilizada nas aspirações e na coleta com armadilha de Shannon. Ao fundo observa-se terreno montanhoso ocupado por pastagem. As coletas de formas imaturas foram feitas no brejo visto no primeiro plano.

Figura 41. Armadilha de Shannon instalada na borda da mata residual localizada na estação de coleta do Canteiro de Obras, no período pré-inundação.

Figura 42. Aspirador elétrico em operação na margem do brejo da estação de coleta do Canteiro de Obras, no período pré-inundação.

Figura 43. Coleta de mosquitos imaturos com concha entomológica em vala na margem do caminho na estação do Canteiro de Obras, no período pré-inundação.........................................................98

Figura 44. Equipe em operação de coleta de mosquitos imaturos, mediante o uso de um barco, na margem direita do lago recém-formado, em posição oposta à estação Córrego Anhumas.

Quadro 1. Fauna de culicídeos em todas as estações investigadas, no período de setembro de 2002 a outubro de 2007, utilizando-se as técnicas de coleta de: armadilha de Shannon, aspirador e concha

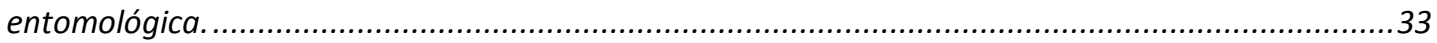
Quadro 2. Fauna de culicídeos segundo as estações investigadas, no período de setembro de 2002 a outubro de 2007, utilizando-se as técnicas de coleta de: armadilha de Shannon, aspirador e concha entomológica.

Tabela 1. Riqueza de espécies segundo o período, a estação de coleta e a técnica de coleta.

Tabela 2. Índice de Margalef $(D)$ dos períodos pré e pós enchimento, segundo método e estação de

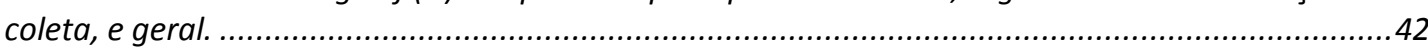
Tabela 3. Dominância (Índice de Simpson) dos períodos Pré e Pós enchimento, segundo método e estação de coleta, e geral. ....44 Tabela 4. IAEP na estação de coleta Canteiro de Obras obtida nas coletas com concha entomológica para avaliação de formas imaturas, no monitoramento da Usina Hidrelétrica de Ourinhos, nos períodos pré e pós enchimento do reservatório. 45 Tabela 5. IAEP na estação de coleta Sítio Caiçara obtida nas coletas com concha entomológica para avaliação de formas imaturas, no monitoramento da Usina Hidrelétrica de Ourinhos, nos períodos pré e pós enchimento do reservatório.

Tabela 6. IAEP na estação de coleta Córrego Anhumas obtida nas coletas com concha entomológica para avaliação de formas imaturas, no monitoramento da Usina Hidrelétrica de Ourinhos, nos períodos pré e pós enchimento do reservatório. Tabela 7. IAEP na estação de coleta Canteiro de Obras obtida nas coletas com aspirador elétrico, no monitoramento da Usina Hidrelétrica de Ourinhos, nos períodos pré e pós enchimento do reservatório.

Tabela 8. IAEP na estação de coleta Sítio Caiçara obtida nas coletas com aspirador elétrico, no monitoramento da Usina Hidrelétrica de Ourinhos, nos períodos pré e pós enchimento do reservatório

Tabela 9. IAEP na estação de coleta Córrego Anhumas obtida nas coletas com aspirador elétrico, no monitoramento da Usina Hidrelétrica de Ourinhos, nos períodos pré e pós enchimento do reservatório.

Tabela 10. IAEP na estação de coleta Canteiro de Obras obtida nas coletas com armadilha de Shannon, no monitoramento da Usina Hidrelétrica de Ourinhos, nos períodos pré e pós enchimento do reservatório. 
Tabela 11. IAEP na estação de coleta Sítio Caiçara obtida nas coletas com armadilha de Shannon, no monitoramento da Usina Hidrelétrica de Ourinhos, nos períodos pré e pós enchimento do

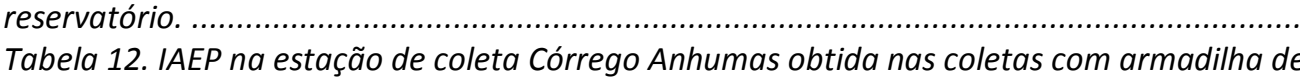
Shannon, no monitoramento da Usina Hidrelétrica de Ourinhos, nos períodos pré e pós enchimento do reservatório.

Tabela 13. IAEP na estação de coleta Canteiro de Obras, obtida pelas três técnicas de coleta, no monitoramento da Usina Hidrelétrica de Ourinhos, nos períodos pré e pós enchimento do reservatório.

Tabela 14. IAEP na estação de coleta Sítio Caiçara obtida pelas três técnicas de coleta, no monitoramento da Usina Hidrelétrica de Ourinhos, nos períodos pré e pós enchimento do reservatório.

Tabela 15. IAEP na estação de coleta Córrego Anhumas obtida pelas três técnicas de coleta, no monitoramento da Usina Hidrelétrica de Ourinhos, nos períodos pré e pós enchimento do reservatório.

Tabela 17. Número de mosquitos segundo as espécies coletados com concha entomológica na fase imatura, no Sítio Caiçara, durante o monitoramento da hidrelétrica de Ourinhos, no período de setembro de 2002 a outubro de 2007.

Tabela 16. Número de mosquitos segundo as espécies coletados com concha entomológica na fase imatura, no Canteiro de Obras, durante o monitoramento da hidrelétrica de Ourinhos, no período de setembro de 2002 a outubro de 2007.

Tabela 18. Número de mosquitos segundo as espécies coletados com concha entomológica na fase imatura, no Córrego Anhumas, durante o monitoramento da hidrelétrica de Ourinhos, no período de setembro de 2002 a outubro de 2007.

Tabela 19. Número de mosquitos segundo as espécies coletados com aspirador no Canteiro de Obras, durante o monitoramento da hidrelétrica de Ourinhos, no período de setembro de 2002 a outubro de 2007......

Tabela 20. Número de mosquitos segundo as espécies coletados com aspirador no Sítio Caiçara, durante o monitoramento da hidrelétrica de Ourinhos, no período de setembro de 2002 a outubro de 2007.

101

Tabela 21. Número de mosquitos segundo as espécies coletados com aspirador no Córrego Anhumas, durante o monitoramento da hidrelétrica de Ourinhos, no período de setembro de 2002 a outubro de 2007 101

Tabela 22. Número de mosquitos segundo as espécies coletados com armadilha de Shannon no Canteiro de Obras, durante o monitoramento da hidrelétrica de Ourinhos, no período de setembro de 2002 a outubro de 2007.

Tabela 23. Número de mosquitos segundo as espécies coletados com armadilha de Shannon no Sítio Caiçara, durante o monitoramento da hidrelétrica de Ourinhos, no período de setembro de 2002 a outubro de 2007.

Tabela 24. . Número de mosquitos segundo as espécies coletados com armadilha de Shannon no Córrego Anhumas, durante o monitoramento da hidrelétrica de Ourinhos, no período de setembro de 2002 a outubro de 2007.

Tabela 25. Número de mosquitos segundo as espécies coletados no Canteiro de Obras, durante o monitoramento da hidrelétrica de Ourinhos, no período de setembro de 2002 a outubro de 2007.. 105 Tabela 26. . Número de mosquitos segundo as espécies coletados no Sítio Caiçara, durante o monitoramento da hidrelétrica de Ourinhos, no período de setembro de 2002 a outubro de $2007 . .106$ Tabela 27. Número de mosquitos segundo as espécies coletados no Córrego Anhumas, durante o monitoramento da hidrelétrica de Ourinhos, no período de setembro de 2002 a outubro de 2007. .107 


\section{Introdução}

\subsection{Energia}

Energia, ar e água são ingredientes essenciais à vida humana. Nas sociedades primitivas seu custo era praticamente zero. A energia era obtida da lenha das florestas para aquecimento e atividades domésticas, como cozinhar. Aos poucos, porém o consumo de energia foi crescendo tanto que outras fontes se fizeram necessárias. Durante a Idade Média, as energias dos cursos d’água e dos ventos foram utilizadas, mas sem conseguir suprir todas as necessidades da população crescente, sobretudo nas cidades. A partir da Revolução Industrial, foi sendo cada vez mais necessário o uso de carvão, petróleo e gás, que tem um custo elevado econômico para a produção e transporte, além de um grande custo ambiental (Goldemberg e Lucon, 2007).

Segundo Odum (1988), as cidades e outros conglomerados humanos representam em termos ecológicos, ecossistemas consumidores (importadores) de recursos que necessitam de constante abastecimento de matéria e energia provenientes de ambientes vizinhos para sua manutenção.

Diversas cidades do mundo se expandem de forma desordenada e caótica. Um exemplo disso é a cidade de São Paulo que a partir dos anos 50 do último século passou por enorme expansão, tendo atualmente por volta de 10,9 milhões de habitantes. Estando inserida em meio à região mais urbanizada do país sofre, portanto, o estresse ambiental próprio das grandes metrópoles, como: a pavimentação 
excessiva, as ilhas de calor, a poluição, o ruído, o adensamento populacional, além de problemas sociais como a exclusão, a violência, a falta de saneamento e saúde, e demais desafios típicos desse ambiente. Com esse rápido crescimento urbano surge uma crescente demanda de recursos energéticos.

O avanço no desenvolvimento humano está fortemente relacionado com o consumo de energia. Em países onde o consumo anual per - capita de energia é inferior a 1 TEP (tonelada equivalente de petróleo), as taxas de mortalidade infantil e analfabetismo são elevadas e a expectativa de vida é baixa. Estudos recentes mostram uma tendência ao crescimento da demanda energética de $4 \%$ em conseqüência da melhoria de qualidade de vida nos países em desenvolvimento (Martins, 2004).

Após a época do “milagre econômico”, ocorreu no Brasil certa desaceleração no crescimento do Produto Interno Bruto (PIB), da produção de energia primária e do consumo de eletricidade. Nos últimos trinta anos, o aumento da produção de energia primária no Brasil tem acompanhado de perto o crescimento do PIB, mas o consumo de eletricidade tem aumentado mais rapidamente, em razão da eletrificação crescente do país e da instalação de indústrias eletrointensivas, como as de alumínio (Goldemberg e Lucon, 2007).

O consumo total de energia elétrica no Brasil passou de 18.346 GWh em 1960 para 304.634 GWh em 2000, o que representa uma taxa de crescimento média anual no período de 7,35\%. Os anos que mais contribuíram para estes números foram os que vieram logo após a implementação do Plano Real, isto é, nos anos 1995 e 1996 (Schmidt e Lima, 2004). 
A demanda por energia para movimentar a economia nacional tende a apresentar um crescimento com a implantação do PAC (Plano de Aceleração do Crescimento) que visa o crescimento econômico do país e o acesso a energia elétrica a todos, desta maneira prevê a necessidade de uma obtenção de energia crescente, admitindo em seu projeto um investimento em infra-estrutura nessa área de cerca de 274,80 bilhões de reais para o período de 2007 - 2010 e com isso ter até 2010 uma geração de 12.400 MWh. ${ }^{1}$

\subsection{Tipos de energia}

Entre as formas de obtenção de energia existem as usinas termoelétricas que são movidas com a utilização de combustíveis fósseis (gás natural, carvão mineral, etc.), porém estas liberam na atmosfera, durante seu processo de produção grande volume de $\mathrm{CO}_{2}$ e outros poluentes, gerando uma energia classificada como suja, devido ao impacto de sua produção.

O aumento da demanda energética, a possibilidade de redução da oferta de combustíveis convencionais causada por crises políticas em regiões produtoras e a crescente preocupação com a preservação do meio ambiente vem levado o homem a pesquisar, desenvolver e utilizar fontes alternativas de energia menos poluentes, renováveis e que produzam pouco impacto ao ambiente como a energia eólica, a oceânica (marés e ondas), a solar, a hidrelétrica e a controversa energia nuclear.

\footnotetext{
${ }^{1}$ http://www.brasil.gov.br/pac/infra_estrutura/energia/
} 
Essas formas de obtenção de energia ainda apresentam fatores limitantes para sua utilização em larga escala, sendo de ordem econômica (alto custo de produção), ambiental (necessidade de amplas áreas e diferentes tipos e níveis de poluição gerados), ou social (realocação de pessoas e risco de acidentes e a saúde).

\subsection{O modelo energético brasileiro e seus impactos}

O Brasil possui uma rede hidrográfica vasta e densa, com alto potencial dos cursos de água para produção de energia gerada a partir da construção de barragens. Desenvolve tecnologias e mão-de-obra altamente especializadas na construção de usinas hidrelétricas. As principais bacias hidrográficas do Sul e do Sudeste do Brasil foram reguladas pela construção de inúmeros reservatórios para a geração de hidreletricidade, o que se constituiu em um significativo impacto na qualidade e na quantidade das águas dessas bacias, mas ao mesmo tempo suportou o desenvolvimento econômico e o progresso da infra-estrutura, principalmente nessas regiões (Tundisi, 2007).

Os empreendimentos hidrelétricos causam alterações sociais e ambientais relacionados à remoção de populações ribeirinhas, à modificação da vazão do rio barrado, à flora e à fauna (Guimarães e col., 1997). Como por exemplo, a tensão resultante do gerenciamento dos estudos de impactos e da ineficiência dos processos de licenciamento, mencione-se que, no início de 2007, estavam em andamento no Ministério Público Federal cerca de 70 processos (ações civis públicas, inquéritos e 
outros procedimentos) envolvendo licenciamento, implantação ou operação de barragens (Vainer, 2007).

Bermann (2007) diz que existem empreendimentos hidrelétricos que têm se revelado conflituosos, no cenário internacional e também no Brasil. Essa constatação pode ser estabelecida a partir de critérios que identificam os problemas físicoquímico-biológicos decorrentes da implantação e da operação de uma usina hidrelétrica, e da sua interação com as características ambientais do seu lócus de construção. Entretanto quando o empreendimento é bem planejado, as compensações e medidas preventivas aplicadas podem reduzir e diluir os conflitos.

Dentre as principais questões ambientais relacionadas às usinas hidrelétricas, cabe destacar: alteração do regime hidrológico, podendo comprometer as atividades a jusante do reservatório; possível comprometimento da qualidade das águas, em razão do caráter lêntico do reservatório, dificultando a decomposição dos rejeitos e efluentes; assoreamento dos reservatórios, em virtude do descontrole no padrão de ocupação territorial nas cabeceiras dos reservatórios, submetidos a processos de desmatamento e retirada da mata ciliar; emissão de gases de efeito estufa, particularmente o metano, decorrente da decomposição da cobertura vegetal submersa definitivamente nos reservatórios; aumento do volume de água no reservatório formado, com conseqüente aumento da pressão sobre o solo e subsolo pelo peso da massa de água represada, em áreas com condições geológicas desfavoráveis (por exemplo, terrenos cársticos), provocando sismos induzidos (Ferrete, 2004); problemas de saúde pública, pela formação dos remansos nos reservatórios e a decorrente proliferação de vetores transmissores de patógenos de doenças endêmicas (Natal, 2001; Gomes e col., 2007); dificuldades para assegurar o 
uso múltiplo das águas, em razão do caráter histórico de priorização da geração elétrica em detrimento dos outros possíveis usos como irrigação, lazer, piscicultura, entre outros.

Essas questões devem ser analisadas em um processo que deve ser público e transparente, alheio a pressões do empreendedor. Do outro lado, temos ações de ONGs, grupos de ambientalista e outros movimentos públicos que apresentam forte resistência à implantação de novas barragens, principalmente as de grande porte, entre esses grupos temos o MAB (Movimento dos Atingidos por Barragens) que prega ações de protesto e resistência, além de ideais como a repotencialização das hidrelétricas já existentes no país e a utilização de outra forma de obtenção de energia com menor impacto ${ }^{2}$,

\subsection{Hidrelétricas e Culicídeos}

Desde o século XIX, há registros de influência sobre a população de culicídeos por alterações ambientais quando da formação de hidrelétricas (Müller, 1995).

A intrusão do homem em ecossistemas naturais e as mudanças ambientais resultantes modificam habitats estabelecidos e criam condições para novas configurações ecológicas. Estas mudanças provem oportunidades para o estabelecimento da população de mosquitos vetores e da transmissão de patógenos

\footnotetext{
${ }^{2}$ http://www.mabnacional.org.br/
} 
por eles veiculadas. Podem também alterar a diversidade das populações de vetores pré-existentes no local.

Desmatamento intensivo e extensivo, obstrução parcial de rios, estradas sem sistema de drenagem adequado, formação de lagos artificiais são exemplos de mudanças ambientais que podem resultar na alteração da densidade de vetores, favorecendo ou não doenças endêmicas cujos agentes são transmitidos por vetores (Natal, 2001).

Como resultado do desmatamento, construção do reservatório e a introdução de populações humanas em locais onde o inseto vetor é endêmico, o risco de epidemias aumentou (Tadei e cols., 1998).

No estudo de Guimarães (2004) observaram-se três fases distintas da população de anofelinos relacionadas com o processo de construção e funcionamento da hidrelétrica de Serra da Mesa, em Goiás. Num primeiro momento com o desmatamento intensivo para a construção e conseqüente exposição dos criadouros ao Sol e as águas barrentas, não foram encontrados anofelinos. Com o reflorestamento das áreas marginais e o enchimento do reservatório, o que gerou uma menor variação do nível da água e sombreamento das margens, as capturas de Anopheles aumentaram. Após um longo período de seca com a usina operando em sua capacidade máxima, o nível do reservatório recuou, expondo bancos de areia nas margens e a freqüência de anofelinos decresceu.

Grandes obras de engenharia como usinas hidrelétricas, auto-estradas e ferrovias, assim como projetos de mineração e agropecuárias, por mais bem planejados que venham a ser, além de imporem uma pressão sobre os limites dos ambientes silvestres, sempre acabam por gerar fluxos migratórios desordenados que 
trazem muitas vezes pessoas infectadas de regiões endêmicas, que ao entrar em contato com a fauna Culicidae local podem vir a gerar surtos e epidemias (Guimarães, 2004) ou o processo inverso pode também ocorrer, ou seja, essas pessoas atraídas pela oportunidade de emprego migram para regiões de endemia e após um período retornam à sua localidade natal levando consigo o patógeno e o introduzindo.

As modificações ambientais podem alterar a dinâmica populacional dos culicídeos, colocando a população humana local ou migrante sob risco de contrair doenças veiculadas por esses mosquitos. Os mosquitos de comportamento antropofílico constituem possível condição de transmissibilidade de patógenos e exposição ao incômodo provocado pela atividade de repasto, quando em grande abundância (Natal, 1998).

Nesse histórico recente, incluem-se a construção das usinas hidrelétricas de Balbina (Amazonas), Samuel (Rondônia) e principalmente Tucuruí (Pará). Em todos esses casos, a formação dos grandes reservatórios nas áreas de montante foi acompanhada da introdução desordenada de populações humanas, principalmente de garimpeiros, vindas de regiões endêmicas para doenças transmitidas por mosquitos, dentre as quais a malária (Tadei e cols., 1998; Quintero e cols., 1996; Monosowski, 2006).

Estudos ecológicos de mosquitos apresentam importância ao levantamento de informações sobre a adaptabilidade ou vulnerabilidade destes as mudanças ambientais. Algumas espécies de culicídeos podem ser utilizadas como bioindicadores da qualidade ambiental por meio de levantamentos de dados acerca 
das variações na densidade populacional ou até mesmo a ausência de algumas espécies estritamente silvestres (Dorvillé, 1996; Forattini e Massad, 1998).

A atuação do homem, modificando o ambiente natural do seu entorno, e o processo de urbanização podem proporcionar condições propicias à proliferação de culicídeos, considerando a capacidade adaptativa deste grupo às diversas alterações ambientais, pode-se esperar mudanças em relação aos quadros epidemiológicos das infecções transmitidas por suas espécies vetoras (Forattini e Massad, 1998).

Diversos estudos trouxeram contribuições para o conhecimento a respeito dos mosquitos do Brasil, evidenciando o comportamento desses frente às alterações do ambiente como o desmatamento (Forattini e cols., 1986), as formações de barragens para fins de geração de energia (Falavigna-Guilherme e cols., 2005, Luz e Lourençode-Oliveira, 1996, Marcondes e col. 2006, Natal e col., 1991). Um exemplo dessa relação entre a pressão antrópica no ambiente natural e o comportamento do vetor é a malária na região Amazônica que tem sido relacionada à forma de utilização dos espaços nos projetos de colonização e observando-se a consequente adaptação do Anopheles (Nys.) darlingi e seu papel como vetor (Tadei e cols., 1998, Vittor e cols., 2006).

Na região ocidental do Estado do Paraná, construiu-se a usina de Itaipu e assim houve o aumento de criadouros de anofelinos em municípios da margem esquerda após a formação do reservatório, isso teria contribuído para a ocorrência de casos de malária na região (Falavigna-Guilherme e cols., 2005; Consolim e col. 1991). Nesse caso, o registro do surto esteve relacionado à migração de indivíduos infectados da área endêmica e a fatores socioeconômicos que determinaram esses movimentos populacionais para a referida região. 
De acordo com o Catálogo Internacional de Arbovírus, foram registrados 535 vírus, dos quais 266 isolados em mosquitos, sendo que vários desses vírus estão relacionados com grande número de epidemias registradas no mundo (Karabastos, 1985). Várias espécies de mosquitos revelaram-se potenciais vetores do Vírus do Nilo Ocidental (VNO) que em 1999 emergiu nos Estados Unidos. Entre elas figuram Aedes albopictus, Culex quinquefasciatus e outras (Turell e col., 2001; Luna e col., 2003). A partir daí, o vírus se dispersou por todo o território americano e, para o norte em direção ao Canadá e, ao sul para a América Central (Natal e Ueno, 2004). Chama-se a atenção para um surto já ocorrido na Argentina, onde o vírus foi isolado em eqüinos (Morales e cols., 2006) e comunicada a detecção do vírus em humanos pelo Ministério de Salud de la Nación em Novembro de 2006, o que atesta a possibilidade do vírus se dispersar para o Brasil em um curto espaço de tempo, fazendo-se necessária constante vigilância entomológica.

Em São Paulo, levantamentos da fauna de culicídeos foram realizados em hidrelétricas no passado (Correa e cols. 1966) e, recentemente, buscou-se fornecer conhecimento das espécies presentes nas áreas das principais bacias represadas (Natal e cols. 1995; Natal e cols. 1998; Paula e Gomes 2007), porém poucos relatos descrevem projetos para monitorar as espécies de mosquitos presentes nas áreas afetadas (Natal 2001).

Se por um lado as hidrelétricas suprem, em parte, as necessidades energéticas do país, um dos impactos dentre tantos, é o possível aumento da fauna de artrópodes vetores de patógenos, como por exemplo, mosquitos do gênero Anopheles (vetores de plasmódios) (Consolim e col. 1991), o gênero Aedes (vetores dos vírus da dengue, da febre amarela e outros arbovírus), o gênero Culex (vetor de filárias) e também o 
aumento de contato com tais artrópodes em diferentes fases do empreendimento como o gênero Haemagogus (vetor do vírus da febre amarela), na fase de desmatamento e inicio da construção e os Triatomíneos (vetores do Trypanosoma cruzi), durante a construção. E conforme observações de Thiengo e col. (2005) houve também o aumento de caracóis (moluscos que servem de hospedeiros intermediários imprescindíveis para efetivação do ciclo de vida do agente da esquistossomose) em área de reservatório. 


\section{Justificativa}

Estudos sobre a ecologia de culicídeos, em áreas de influência de hidrelétricas, realizados nos períodos antes, durante e após a construção e enchimento do reservatório fazem-se necessários para uma análise acerca das alterações sofridas por essa população em seus processos ecológicos, para previsão de tendências na abundância dos mosquitos e dá suporte aos programas de controle. Os processos de construção e posterior operação do reservatório interferem na evolução das comunidades de organismos aquáticos, bem como nas características químico-físicas dos sedimentos e da água, podendo ocasionar impactos na Saúde Pública, como surtos e epidemias de doenças cujos agentes são veiculados por artrópodes (Quintero e col. 1996). No entanto, os procedimentos necessitam de uniformidade e padronização entre os diferentes estudos realizados no território nacional, para que se possa comparar e analisar os dados adequadamente, além de se obter um quadro abrangente do efeito da intervenção sobre a diversidade dos mosquitos. 


\section{Objetivos}

\subsection{Objetivo Geral}

- Descrever a diversidade de mosquitos (Culicidae) em área impactada pela Hidrelétrica Ourinhos, SP/PR.

\subsection{Objetivos específicos}

- Descrever a diversidade das populações de culicídeos por meio de índices de riqueza, dominância, similaridade e diversidade nos períodos pré e pós enchimento;

- Detalhar a composição faunística de mosquitos das estações de coleta, comparando-as entre os períodos pré e pós enchimento.

- Discutir a importância epidemiológica das espécies dominantes e/ou mais abundantes, ressaltando os potenciais vetores dentre a riqueza de espécies. 


\section{Materiais e Métodos}

\section{1. Área de estudo}

A usina hidrelétrica de Ourinhos (Anexo I; Figs. 32 e 33) foi construída pela Companhia Brasileira de Alumínio (CBA) com o aproveitamento das águas do rio Paranapanema, localizado entre as cidades de Ourinhos, SP, e Jacarezinho, PR. Trata-se de uma hidrelétrica que se enquadra no modelo de auto-produção, ou seja, ela destina-se ao consumo particular da empresa.

Sua primeira unidade entrou em operação comercial em dezembro de 2005, e tem o período de concessão de 35 anos, iniciado em julho de 2000. A potência instalada é de 44 MW e a energia gerada é de 207 mil MWh/ano.

Os municípios de Ourinhos, Canitar e Chavantes, em São Paulo, e Jacarezinho e Ribeirão Claro, no Paraná, tiveram inundação de trechos de suas áreas pelo reservatório da usina hidrelétrica Ourinhos (Tabela 1). A área total inundada pelo reservatório da usina é 5,09 km² no território dos cinco municípios. ${ }^{3}$

Tabela 1: Percentual da área inundada pelo reservatório da UHE de Ourinhos por município.

\begin{tabular}{|c|c|c|}
\hline Município & UF & Área Inundada (\%) \\
\hline Ourinhos & SP & 1,68 \\
\hline Canitar & SP & 11,34 \\
\hline Chavantes & SP & 43,86 \\
\hline Jacarezinho & PR & 13,05 \\
\hline Ribeirão Claro & PR & 30,04 \\
\hline Área Total & 100 \\
\hline
\end{tabular}

\footnotetext{
${ }^{3}$ http://www.aneel.gov.br/arquivos/PDF/boletim211.htm
} 


\subsection{Dados da Fauna}

Os dados a cerca da fauna de culicídeos foram fruto de um trabalho de monitoramento ambiental de culicídeos entre 2002 e 2007. Os procedimentos adotados nas coletas estão descritos a seguir.

\subsubsection{Trabalho de Campo}

Foram definidas três estações de coleta (Anexo I; Fig. 34) que foram usadas para a avaliação de impactos nos períodos pré e pós inundação do reservatório. Tais estações foram denominadas: Canteiro de Obras (SP), Sítio Caiçara (SP) e Córrego Anhumas (PR), representadas respectivamente nas figuras dos Anexos I (Figs 35, 36 e 37.) e II (Figs 38, 39 e 40)

A estação de coleta Canteiro de Obras está alocada a meio quilômetro à jusante do eixo da barragem. As outras duas estações, à montante do referido eixo, foram escolhidas intencionalmente para que permanecessem nas bordas do futuro reservatório. Assim, no período anterior à inundação essas localidades estavam na confluência da várzea com os terrenos mais elevados, e após a inundação passaram a se localizar na margem imediata do lago. A escolha desses pontos permitiu a continuidade das coletas de mosquitos na fase pré e pós inundação, sem haver a necessidade de mudanças dos locais de pesquisa, que seriam motivadas pela própria inundação.

Sob o aspecto paisagístico, as estações de coleta apresentavam vários ambientes, tais como: matas residuais, resquícios de matas de galeria, brejos e 
charcos ocupados por gramíneas ou taboas, lugares de vegetação aberta constituídos por campos e pastos e áreas de ocupação humana como residências, galinheiros e pomares.

Para as três estações de coleta, estabeleceu-se a rotina de campo discriminada a seguir. A permanência da equipe no campo foi de três dias. Em cada um desses dias era implantada a mesma atividade de captura, a qual foi padronizada ao longo de toda pesquisa.

\section{a. Mosquitos adultos}

Mosquitos adultos foram capturados mediante o uso de aspirador com bateria (Nasci, 1981) e armadilha de Shannon (Shannon, 1939).

A utilização de aspiradores elétricos foi para a obtenção de mosquitos em abrigos naturais, ou seja, em repouso na vegetação ou em pontos úmidos e escuros, onde se ocultam durante o dia. Esse tipo de captura teve duração de 15 minutos em cada um dos pontos de coleta, depois os mosquitos foram mortos com clorofórmio. (Anexo III - Figura 42)

Em cada estação de coleta, a partir do início do crepúsculo vespertino e nas três horas a seguir, esteve em operação uma armadilha de Shannon, com luz de lampião a gás e com dois indivíduos coletores (Anexo III - Figura 41). O ponto de coleta foi definido como o local com cobertura arbórea, que sofria a menor influência da iluminação elétrica, ou seja, o local mais escuro. Os operadores, munidos de capturadores (tubos com clorofórmio), efetuaram as coletas de mosquitos nas laterais internas e externas e no teto da armadilha, sendo o material obtido, segregado por horário. 
Os mosquitos adultos foram acondicionados em caixas entomológicas preparadas com naftalina, rotuladas e enviadas ao Laboratório de Entomologia em Saúde Pública (LESP) da Faculdade de Saúde Pública da USP, para a identificação.

\section{b. Formas imaturas de mosquitos}

Investigou-se o ambiente de borda do lago e brejos, na tentativa de localização de coleções de água estagnada, propícia ao desenvolvimento de culicídeos. Nas quatro últimas coletas, foi disponibilizado um barco para permitir o acesso a pontos do lago inacessíveis por terra. Uma vez localizados os criadouros, procedia-se a coleta, utilizando-se “conchas entomológicas”. (Anexo III- Figs 43 e 44)

Todo material biológico obtido foi acondicionado vivo em frascos plásticos etiquetados, com água recolhida dos respectivos criadouros, para envio ao LESP.

\subsection{Trabalho de laboratório}

Após o período de campo foram desenvolvidas as seguintes atividades em laboratório.

\section{a. Desenvolvimento das formas imaturas de mosquitos}

No laboratório, cada exemplar coletado (larva ou pupa) foi acompanhado individualmente em pequenos frascos até a emergência do respectivo adulto em mini-pupários, sendo alimentado com ração para peixe macerada. As exúvias das formas imaturas foram recolhidas e acondicionadas em álcool $70 \%$ onde permaneceram até a montagem. Essa foi feita entre lâmina etiquetada e lamínula, 
com bálsamo do Canadá. Os adultos obtidos foram mortos com clorofórmio, quando necessário, fixados em alfinetes entomológicos e etiquetados. Assim, foi estabelecida a correspondência entre a exúvia da forma imatura e o indivíduo adulto, procedimento que permite identificação mais segura.

\section{b. Identificação e catalogação}

A identificação das formas imaturas e adultos, tanto coletados no campo como os emergido em laboratório, até a categoria de espécie, foram realizadas no Laboratório de Culicídeos da Faculdade de Saúde Pública da USP com o auxilio de descrições e chaves taxonômicas conhecidas na literatura de Diptera: Culicidae (Forattini, 2002; Lane, 1953; Consoli e Lourenço-de-Oliveira, 1994). Após serem identificados, os exemplares foram catalogados para confecção de banco de dados.

\subsection{Forma de análise}

Com base nos dados levantados foram montados os bancos de dados utilizando o Excel o qual centralizou todas as informações sobre as identificações. A partir desses bancos, foram realizados os cruzamentos para a organização dos dados para as análises utilizando-se softwares como o Excel, o Software Ecosim 7.0 (Gotelli e Entsminger, 2009) e o Software R 2.5.0 (RDCT, 2007). Foram realizadas as análises faunísticas, baseadas em, Krebs (1978) e Magurran (2004). Estimaram-se os seguintes parâmetros:

Riqueza - (R) - trata-se de indicador qualitativo que revela o número de espécies encontradas. 
Diversidade $\alpha$ - (D) - (Índice de Margalef) foi definida como: D = (S-1)/log e $\mathrm{N}$ (Service, 1993), onde $\mathrm{S}=\mathrm{n}^{\circ}$ de espécies; $\mathrm{N}=\mathrm{n}^{\circ}$ de indivíduos.

Dominância - $(\lambda)$ - (Índice de Símpson) foi definida como: $\lambda=\Sigma\left(\mathrm{n}_{i} *\left(\mathrm{n}_{i^{-}}\right.\right.$ 1))/(N*(N-1)) (Magurran, 2004), onde $\mathrm{n}_{i}=$ número de indivíduos da espécie $i$, e $\mathrm{N}=$ $\mathrm{n}^{\mathrm{o}}$ de indivíduos da amostra.

Abundância - (IAE) - (Índice de Abundância de Espécie) e (IAEP) - (Índice de Abundância de Espécie Padronizado) definidos como: IAE = a+Rj/K; IAEP = cIAE/c-1 (Roberts e Hsi, 1979), onde c = maior ordenamento da tabela +1 (gera-se uma tabela de ocorrência numérica - $1^{\mathrm{a}}$ tab. -, a qual é transformada em outra correspondente - $2^{\mathrm{a}}$ tab. -, segundo a posição de cada espécie; sendo c definido como o maior valor dessa segunda tabela acrescido da unidade; $\mathrm{a}=\mathrm{n}^{0}$ de casas vazias por espécie $\mathrm{X} \mathrm{c}$; $\mathrm{Rj}$ = somatória das casas ou soma das posições (na $2^{\mathrm{a}}$ tab.) por espécie; $\mathrm{K}=\mathrm{n}^{\mathrm{o}}$ de sítios (em estudo espacial) ou de meses (em estudo temporal) de coleta.

Similaridade $-\left(\boldsymbol{I}_{\boldsymbol{J}}\right)$ - (Coeficiente de Similaridade de Jaccard) definida pela equação: $I_{J}=c / a+b-c$, onde $a, b$ e $c$ são número de espécies presentes na amostra A, B e em ambas, respectivamente.

Diversidade $\boldsymbol{\beta}$ - $(\boldsymbol{\beta})$ - (Índice de Magurran) foi definido como: $\beta=(a+b) *(1-$ $I_{J}$ ) (Magurran, 1988), sendo $I_{J}$ o índice de similaridade de Jaccard entre as amostras A e B. 


\section{Resultados}

\subsection{Lista de espécies}

O Quadro 1 mostra a fauna das três estações de coleta aglutinadas em uma única lista. A lista geral das espécies da região durante todo o período de estudo atingiu o valor de 90 táxons, distribuídas em 12 gêneros.

No Quadro 2, a fauna de mosquitos obtida em todas as campanhas de campo com as três técnicas de coleta, envolvendo o período pré-enchimento e o pósenchimento. Nesse quadro os táxons identificados estão dispostos segundo as estações de coleta. O número de espécies ou táxons identificados nas três estações de coleta, Canteiro de Obras (CO), Sítio Caiçara (SC) e Córrego Anhumas (CA), ao longo do estudo, foram respectivamente: 66; 58 e 68, pertencentes a 10; 8 e 11 gêneros. 
Quadro 1. Fauna de culicídeos em todas as estações investigadas, no período de setembro de 2002 a outubro de 2007, utilizando-se as técnicas de coleta de: armadilha de Shannon, aspirador e concha entomológica.

\begin{tabular}{|c|c|c|}
\hline \multicolumn{3}{|c|}{ Fauna Geral de Culicídeos } \\
\hline Ad. (Ady.) squamipennis & Cx. (Cux.) chidesteri & Cx. (Mel.) vaxus \\
\hline Ae. (Och.) crinifer & Cx. (Cux.) coronator & Cx. (Mel.) zeteki \\
\hline Ae. (Och.) fluviatilis & Cx. (Cux.) declarator & Hg. (Con.) leucocelaenus \\
\hline Ae. (Och.) nubilus & Cx. (Cux.) dolosus & Li. durhami \\
\hline Ae. (Och.) scapularis & Cx. (Cux.) dolosus/eduardoi & Ma. (Man.) amazonensis \\
\hline Ae. (Och.) serratus & Cx. (Cux.) eduardoi & Ma. (Man.) flaveola \\
\hline Ae. (Och.) serratus/nubilus & CX. (Cux.) grupo Coronator & Ma. (Man.) humeralis \\
\hline Ae. (Och.) terrens & Cx. (Cux.) lygrus & Ma. (Man.) indubitans \\
\hline Ae. (Stg.) albopictus & Cx. (Cux.) mollis & Ma. (Man.) titillans \\
\hline An. (Ano.) fluminensis & Cx. (Cux.) nigripalpus & Ma. (Man.) wilsoni \\
\hline An. (Ano.) maculipes & Cx. (Cux.) quinquefascistus & Ps. (Gra.) cingulata \\
\hline An. (Nys.) albitarsis s.l & Cx. (Cux.) saltanensis & Ps. (Gra.) confinnis \\
\hline An. (Nys.) argyritarsis & Cx. (Cux.) sp. & Ps. (Jan.) albigenu \\
\hline An. (Nys.) darlingi & $C x .($ Mcx.) imitator & Ps. (Jan.) albipes \\
\hline An. (Nys.) evansae & Cx. (Mel.) aureonotatus & Ps. (Jan.) discrucians \\
\hline An. (Nys.) galvaoi & Cx. (Mel.) bastagarius & Ps. (Jan.) ferox \\
\hline An. (Nys.) lutzii & Cx. (Mel.) contei & Ps. (Jan.) lutzii \\
\hline An. (Nys.) sp & Cx. (Mel.) dunni & Ps. (Pso.) ciliata \\
\hline An. (Nys.) strodei & Cx. (Mel.) dureti & Ps. sp. \\
\hline An. (Nys.) strodei/ rondoni & Cx. (Mel.) flabellifer & Sa. $s p$. \\
\hline An. (Nys.) triannulatus & Cx. (Mel.) grupo A tratus & Ur. (Ura.) geometrica \\
\hline Cq. (Rhy.) albicosta & Cx. (Mel.) idottus & Ur. (Ura.) lowii \\
\hline Cq. (Rhy.) chrysonotum & Cx. (Mel.) ocossa & Ur. (Ura.) mathesoni \\
\hline Cq. (Rhy.) chrysonotum/albifera & Cx. (Mel.) pilosus & Ur. (Ura.) nataliae \\
\hline Cq. (Rhy.) hermanoi & Cx. (Mel.) plectoporpe & Ur. (Ura.) pulcherrima \\
\hline Cq. (Rhy.) juxtamansonia & Cx. (Mel.) rabelloi & Ur. (Ura.) sp. \\
\hline Cq. (Rhy.) nigricans & Cx. (Mel.) ribeirensis & Wy. (Den.) rooti \\
\hline Cq. (Rhy.) shannoni & $C x$. (Mel.) seção Melanoconion & Wy. (Wye.) medioalbipes \\
\hline Cq. (Rhy.) venezuelensis & Cx. (Mel.) sp & Wy. sp. \\
\hline Cx. (Ads.) amazonenzis & Cx. (Mel.) sphatulatus & \\
\hline Cx.(Cux.) bidens & & \\
\hline
\end{tabular}


Quadro 2. Fauna de culicídeos segundo as estações investigadas, no período de setembro de 2002 a outubro de 2007, utilizando-se as técnicas de coleta de: armadilha de Shannon, aspirador e concha entomológica.

\begin{tabular}{|c|}
\hline Canteiro de Obras \\
\hline Ad. (Ady.) squamipennis \\
\hline Ae. (Och.) crinifer \\
\hline Ae. (Och.) fluviatilis \\
\hline Ae. (Och.) scapularis \\
\hline Ae. (Och.) serratus \\
\hline Ae. (Stg.) albopictus \\
\hline An. (Ano.) maculipes \\
\hline An. (Nys.) lutzii \\
\hline An. (Nys.) albitarsis s.l \\
\hline An. (Nys.) argyritarsis \\
\hline An. (Nys.) evansae \\
\hline An. (Nys.) galvaoi \\
\hline An. (Nys.) sp \\
\hline An. (Nys.) strodei \\
\hline An. (Nys.) strodei/ rondoni \\
\hline An. (Nys.) triannulatus \\
\hline Cq. (Rhy.) albicosta \\
\hline Cq. (Rhy.) chrysonotum \\
\hline Cq. (Rhy.) chrysonotum/albifera \\
\hline Cq. (Rhy.) hermanoi \\
\hline Cq. (Rhy.) juxtamansonia \\
\hline Cq. (Rhy.) nigricans \\
\hline Cq. (Rhy.) shannoni \\
\hline Cq. (Rhy.) venezuelensis \\
\hline Cx. (Ads.) amazonenzis \\
\hline Cx.(Cux.) bidens \\
\hline Cx. (Cux.) chidesteri \\
\hline Cx. (Cux.) coronator \\
\hline Cx.(Cux.) declarator \\
\hline Cx. (Cux.) dolosus \\
\hline CX. (Cux.) dolosus/eduardoi \\
\hline$C x .(\mathrm{Cux}$.$) grupo Coronator$ \\
\hline Cx.(Cux.) mollis \\
\hline Cx.(Cux.) nigripalpus \\
\hline Cx. (Cux.) quinquefascistus \\
\hline Cx. (Cux.) sp. \\
\hline CX. (McX.) imitator \\
\hline CX. (Mel.) bastagarius \\
\hline Cx. (Mel.) contei \\
\hline Cx. (Mel.) dunni \\
\hline Cx. (Mel.) dureti \\
\hline Cx. (Mel.) grupo A tratus \\
\hline Cx. (Mel.) ocossa \\
\hline Cx. (Mel.) plectoporpe \\
\hline CX. (Mel.) rabelloi \\
\hline$C x .($ Mel.) ribeirensis \\
\hline Cx. (Mel.) seção Melanoconion \\
\hline Cx. (Mel.) sphatulatus \\
\hline Ma. (Man.) amazonensis \\
\hline Ma. (Man.) humeralis \\
\hline Ma. (Man.) indubitans \\
\hline Ma. (Man.) titillans \\
\hline Ma. (Man.) wilsoni \\
\hline Ps. (Gra.) confinnis \\
\hline Ps. (Jan.) albigenu \\
\hline \begin{tabular}{|l|} 
Ps. (Jan.) ferox \\
\end{tabular} \\
\hline Ps. (Jan.) lutzii \\
\hline Ps. (Pso.) ciliata \\
\hline Sa. sp. \\
\hline Ur. (Ura.) geometrica \\
\hline Ur. (Ura.) lowii \\
\hline Ur. (Ura.) mathesoni \\
\hline \begin{tabular}{|l} 
Ur. (Ura.) nataliae \\
\end{tabular} \\
\hline Ur. (Ura.) pulcherrima \\
\hline Wy. (Den.) rooti \\
\hline Wy. sp. \\
\hline
\end{tabular}

Sítio Caiçara

Ad. (Ady.) squamipennis

Ae. (Och.) crinifer

Ae. (Och.) fluviatilis

Ae. (Och.) nubilus

Ae. (Och.) scapularis

Ae. (Och.) serratus

Ae. (Och.) serratus/nubilus

Ae. (Och.) terrens

Ae. (Stg.) albopictus

An. (Nys.) albitarsis s.l

An. (Nys.) darlingi

An. (Nys.) galvaoi

An. (Nys.) $s p$

An. (Nys.) strodei

An. (Nys.) strodei/ rondoni

An. (Nys.) triannulatus

An. (Nys.) argyritarsis

An. (Nys.) evansae

Cq. (Rhy.) albicosta

Cq. (Rhy.) chrysonotum/albifera

Cq. (Rhy.) hermanoi

Cq. (Rhy.) juxtamansonia

Cq. (Rhy.) nigricans

Cq. (Rhy.) shannoni

Cq. (Rhy.) venezuelensis

Cx. (Cux.) chidesteri

Cx. (Cux.) coronator

Cx. (Cux.) declarator

Cx. (Cux.) dolosus

CX. (Cux.) dolosus/eduardoi

Cx. (Cux.) eduardoi

Cx. (Cux.) grupo Coronator

Cx. (Cux.) lygrus

Cx. (Cux.) mollis

Cx. (Cux.) nigripalpus

Cx. (Cux.) quinquefascistus

Cx. (Cux.) sp.

Cx. (Mel.) aureonotatus

CX. (Mel.) dureti

Cx. (Mel.) flabellifer

Cx. (Mel.) grupo A tratus

Cx. (Mel.) ocossa

Cx. (Mel.) pilosus

CX. (Mel.) ribeirensis

Cx. (Mel.) seção Melanoconion

Cx. (Mel.) sp

CX. (Mel.) vaxus

Ma. (Man.) humeralis

Ma. (Man.) indubitans

Ma. (Man.) titillans

Ma. (Man.) wilsoni

Ps. (Pso.) ciliata

Ps. (Jan.) discrucians

Ur. (Ura.) lowii

Ur. (Ura.) pulcherrima

Ur. (Ura.) sp.

Ur. (Ura.) geometrica

Wy. sp.
Córrego Anhumas

Ad. (Ady.) squamipennis

Ae. (Och.) crinifer

Ae. (Och.) nubilus

Ae. (Och.) scapularis

Ae. (Och.) serratus

Ae. (Och.) terrens

An. (Ano.) fluminensis

An. (Nys.) albitarsis s.l

An. (Nys.) argyritarsis

An. (Nys.) darlingi

An. (Nys.) evansae

An. (Nys.) galvaoi

An. (Nys.) sp

An. (Nys.) strodei

An. (Nys.) strodei

An. (Nys.) triannulatus

Cq. (Rhy.) albicosta

Cq. (Rhy.) chrysonotum/albifera

Cq. (Rhy.) hermanoi

Cq. (Rhy.) juxtamansonia

Cq. (Rhy.) nigricans

Cq. (Rhy.) shannoni

Cq. (Rhy.) venezuelensis

CX. (Cux.) bidens

CX. (Cux.) chidesteri

Cx. (Cux.) coronator

Cx. (Cux.) declarator

Cx. (Cux.) dolosus

$C x$. (Cux.) grupo Coronator

Cx. (Cux.) lygrus

Cx. (Cux.) mollis

$C x$. (Cux.) nigripalpus

CX. (Cux.) quinquefascistus

Cx. (Cux.) saltanensis

Cx. (Cux.) sp.

CX. (McX.) imitator

CX. (Mel.) aureonotatus

$\mathrm{Cx}$. (Mel.) bastagarius

Cx. (Mel.) dureti

Cx. (Mel.) grupo A tratus

Cx. (Mel.) idottus

Cx. (Mel.) ocossa

Cx. (Mel.) pilosus

Cx. (Mel.) plectoporpe

$C X$. (Mel.) ribeirensis

$C x$. (Mel.) seção Melanoconion

Cx. (Mel.) sp.

Cx. (Mel.) vaxus

Cx. (Mel.) zeteki

Hg. (Con.) leucocelaenus

Li. durhami

Ma. (Man.) flaveola

Ma. (Man.) indubitans

Ma. (Man.) titillans

Ma. (Man.) wilsoni

Ps. (Gra.) cingulata

Ps. (Gra.) confinnis

Ps. (Jan.) albigenu

Ps. (Jan.) albipes

Ps. (Jan.) discrucians

Ps. (Jan.) ferox

Ps. (Pso.) ciliata

Ps. sp.

Ur. (Ura.) geometrica

Ur. (Ura.) lowii

Ur. (Ura.) pulcherrima

Ur. (Ura.) sp.

Wy. (Wye.) medioalbipes 


\subsection{Riqueza}

Observando-se a ilustração (Figura 1) que mostra a riqueza de formas imaturas observada em cada ponto de coleta, chama a atenção os valores com tendência de aumento de riqueza na região do Sítio Caiçara no período pós enchimento. No final da série, desse mesmo período, há ascensão brusca no valor de riqueza também do Córrego Anhumas. E outro ponto de destaque é a queda da riqueza em todos os pontos coletados na última coleta (6) do período pré enchimento.

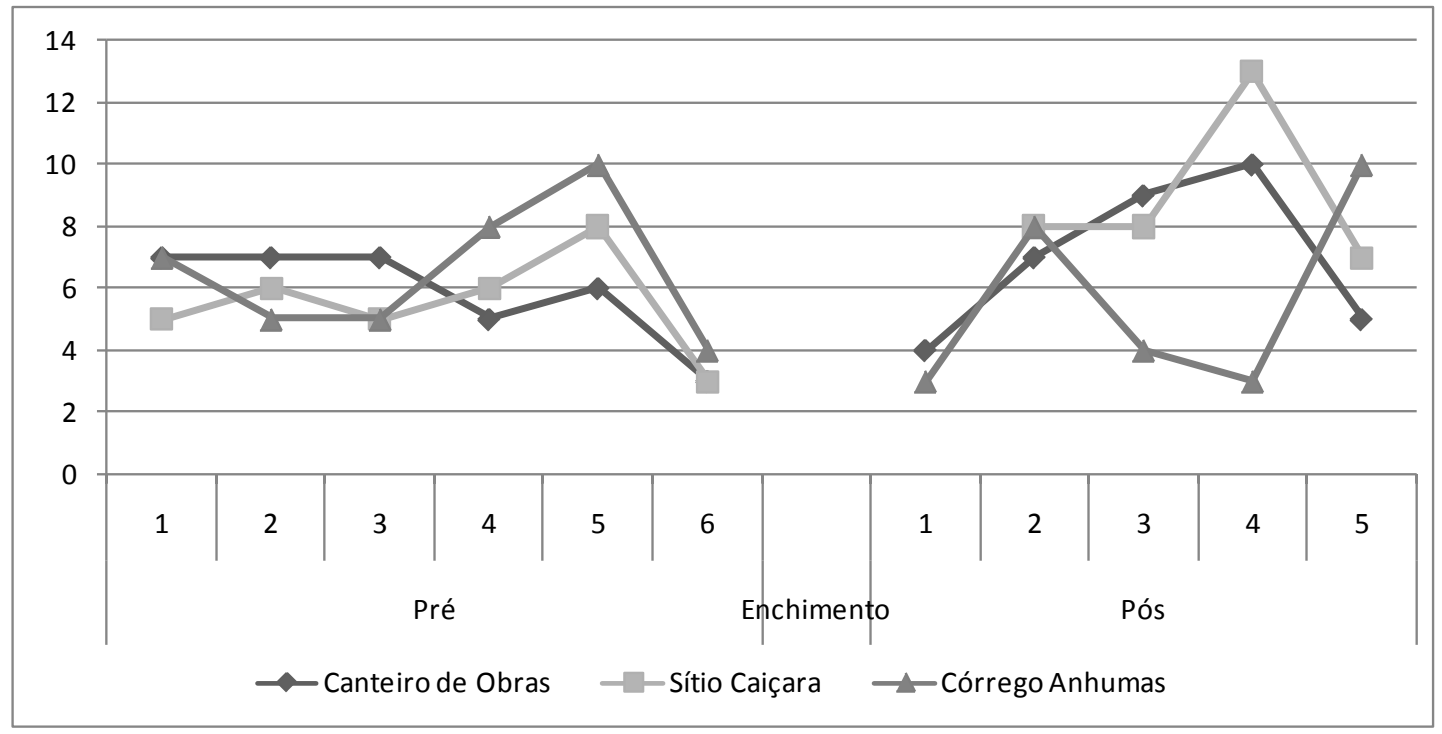

Figura 1. Riqueza segundo estações de coleta e campanhas obtidas nas coletas com concha entomológica para avaliação de formas imaturas, no monitoramento da Usina Hidrelétrica de Ourinhos.

Os dados de Riqueza obtidos na técnica de aspirador elétrico estão ilustrados na Figura 2, a seguir apresentadas. 


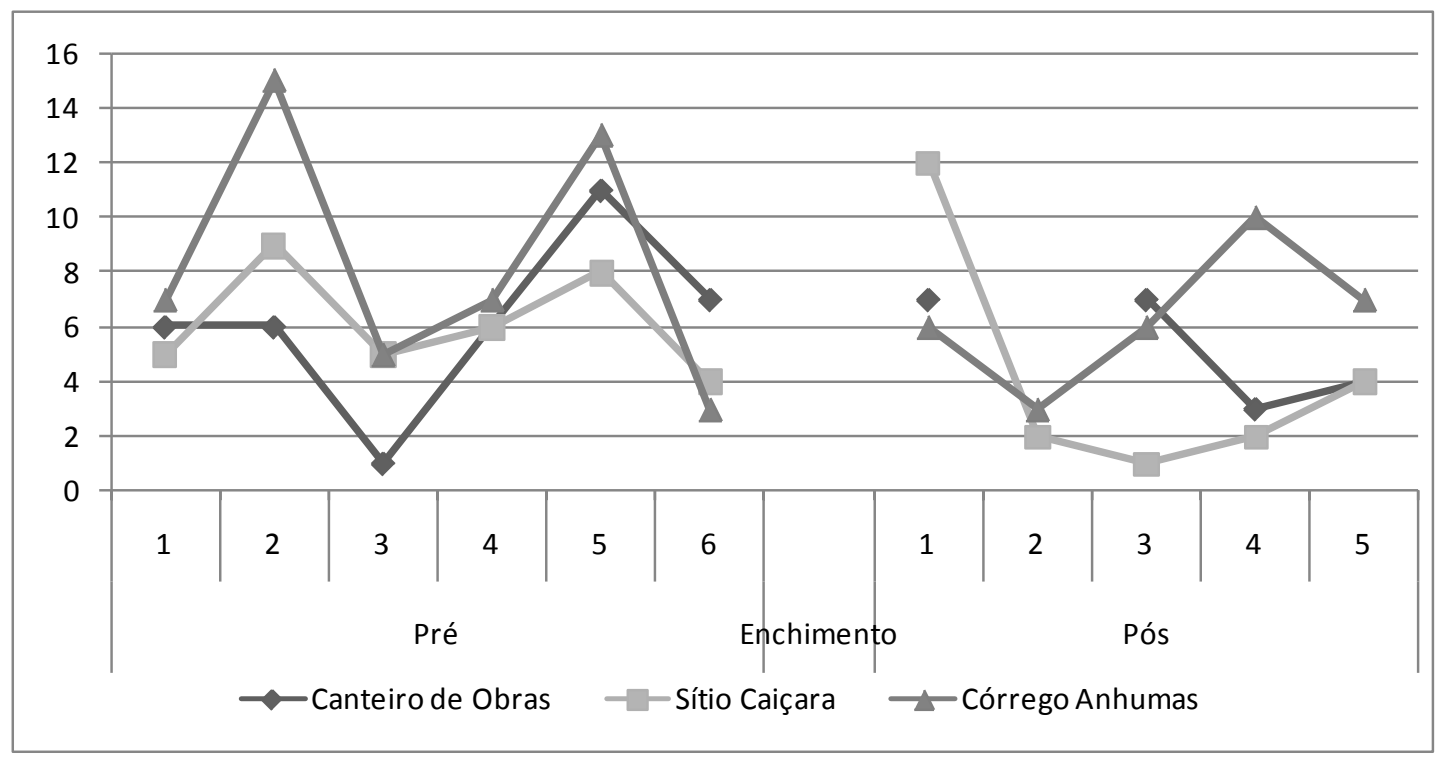

Figura 2. Riqueza segundo estações de coleta e campanhas obtidas nas coletas com aspirador elétrico, no monitoramento da Usina Hidrelétrica de Ourinhos.

Observa-se na ilustração da Figura 2 que a técnica de aspiração gerou, de modo geral, riquezas de valores mais baixos que a armadilha de Shannon, ilustrada na figura anterior. Verificou-se a tendência das riquezas de valores mais baixos concentrarem-se no período pós-inundação. 


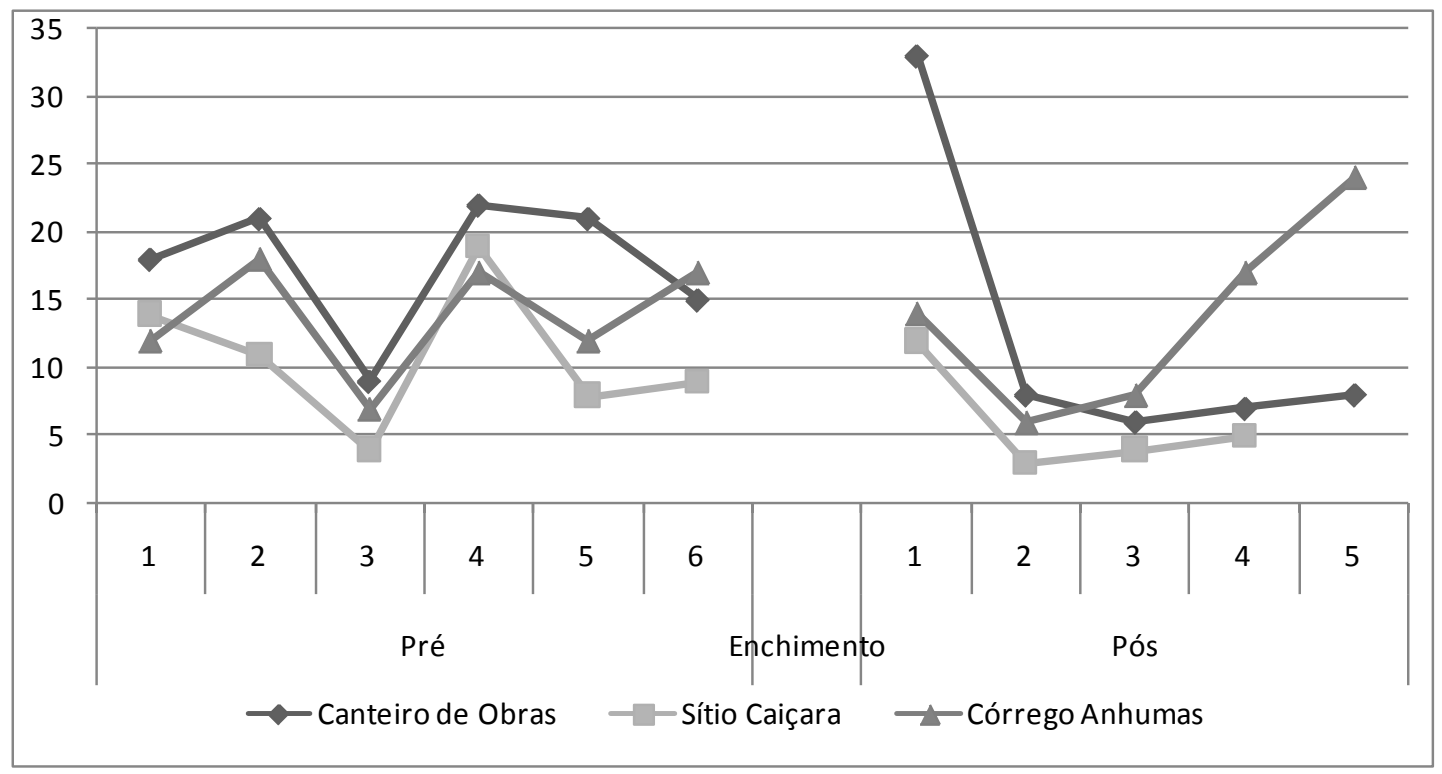

Figura 3. Riqueza segundo estações de coleta e campanhas obtidas nas coletas com armadilha de Shannon, no monitoramento da Usina Hidrelétrica de Ourinhos.

Os dados de Riqueza, obtidos na técnica de coleta por Armadilha de Shannon estão ilustrados na Figura 3, acima.

A observação do gráfico da Figura 3 revela tendência de menores valores de riqueza no Sítio Caiçara durante todo o período de estudo, ao passo que as coletas do Canteiro de Obras, fora da influência direta do lago, geraram dados que corresponderam às maiores riquezas no período pré enchimento, enquanto a riqueza do Córrego Anhumas tendeu a valores intermediários. No final da série do período pós enchimento, as estações Canteiro de Obras e Córrego Anhumas apresentaram diferentes inflexões, sendo que no primeiro desses locais houve acentuada redução do indicador e no segundo o aumento. 


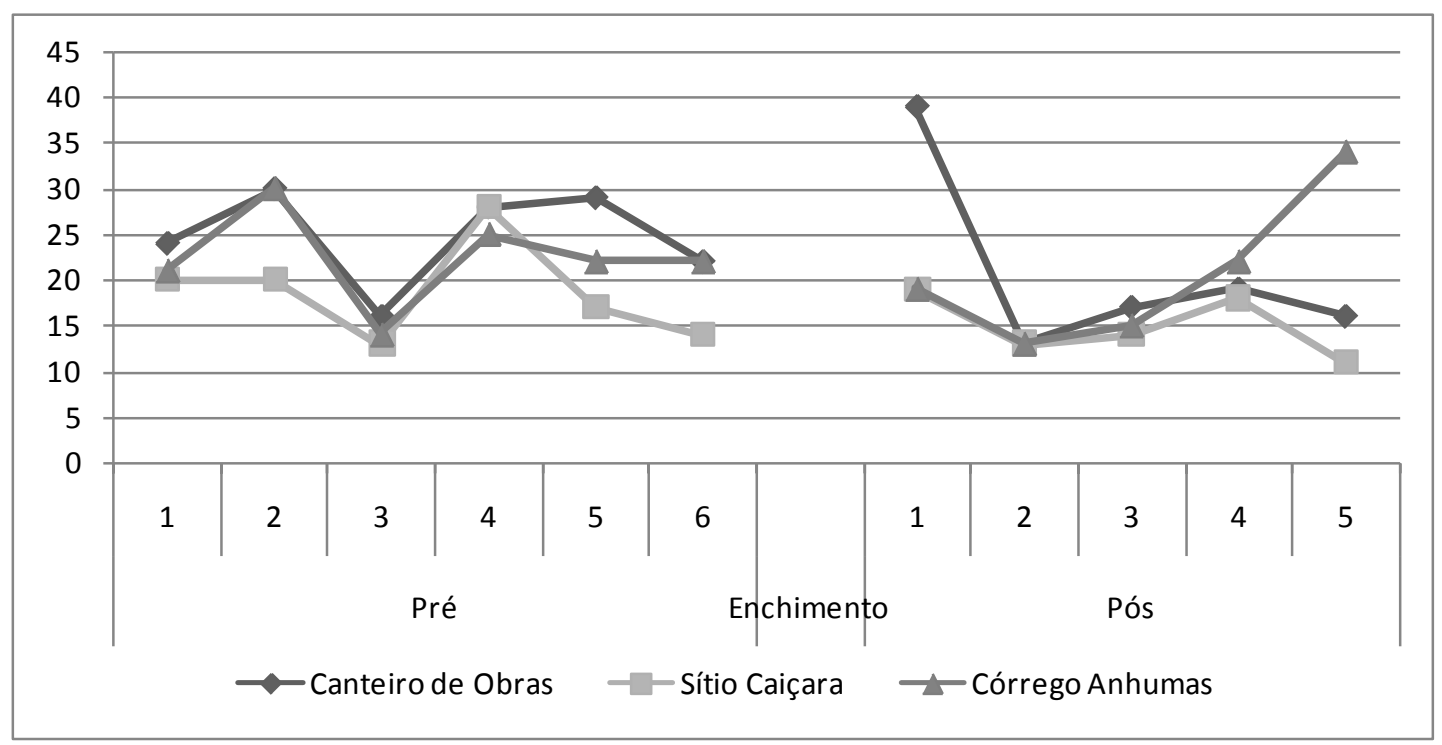

Figura 4. Riqueza segundo estações de coleta e campanhas obtidas no monitoramento da Usina Hidrelétrica de Ourinhos.

A Figura 4 ilustra os valores de Riqueza observados com a utilização conjunta das três técnicas. Verifica-se que o Canteiro de Obras apresentou uma maior riqueza durante as coletas do período pré enchimento quando comparado as outras estações de coleta. No período pós enchimento o comportamento foi similar ao observado nos dados somente da armadilha de Shannon.

Tabela 1. Riqueza de espécies segundo o período, a estação de coleta e a técnica de coleta.

\begin{tabular}{|l|r|r|r|r|r|r|r|r|}
\hline \multirow{2}{*}{ Ponto de Coleta } & \multicolumn{2}{|c|}{ Imaturos } & \multicolumn{2}{c|}{ Aspirador } & \multicolumn{2}{c|}{ Shannon } & \multicolumn{2}{c|}{ Geral } \\
\cline { 2 - 9 } & \multicolumn{1}{c|}{ Pré } & \multicolumn{1}{c|}{ Pós } & \multicolumn{1}{c|}{ Pré } & \multicolumn{1}{c|}{ Pós } & \multicolumn{1}{c|}{ Pré } & \multicolumn{1}{c|}{ Pós } & \multicolumn{1}{c|}{ Pré } & \multicolumn{1}{c|}{ Pós } \\
\hline Canteiro de Obras & 22 & 20 & 24 & 16 & 39 & 36 & 55 & 51 \\
\hline Sítio Caiçara & 15 & 20 & 17 & 16 & 27 & 19 & 42 & 42 \\
\hline Córrego Anhumas & 24 & 15 & 26 & 24 & 36 & 37 & 54 & 46 \\
\hline
\end{tabular}

Quando comparamos as riquezas gerais dos períodos (Tab. 1), observamos uma redução destas entre os períodos pré e pós enchimento para a estação Canteiro de Obras em todas a técnicas utilizadas. As estações Córrego Anhumas e Sítio Caiçara apresentaram aumento da riqueza no período pós em relação ao pré para as 
técnicas de aspirador elétrico (adultos) e concha entomológica (formas imaturas), respectivamente. Quando observamos os dados obtidos pelas três técnicas em conjunto, sobressai a redução nas estações Canteiro de Obras e Córrego Anhumas e a manutenção da riqueza no Sítio Caiçara.

As riquezas observadas nas curvas de rarefação indicam uma redução tênue no período pós em relação ao pré, quando temos o mesmo tamanho de amostra para o Canteiro de Obras e o Córrego Anhumas (Figs. 5 e 7). O Sítio Caiçara apresentou uma riqueza maior no período pós do que no pré enchimento, para uma amostra de mesmo tamanho (Fig. 6).

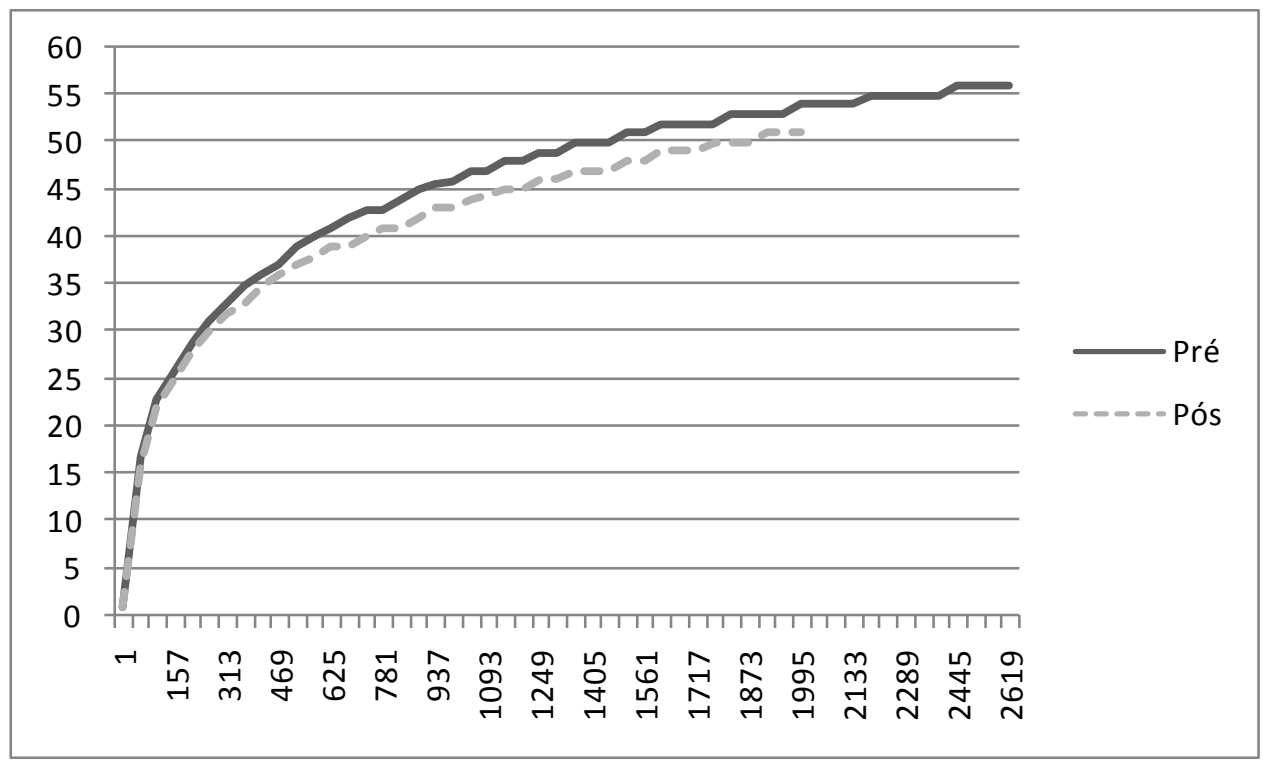

Figura 5. Curva de rarefação, segundo número de indivíduos coletados, para a estação de coleta Canteiro de Obras, no monitoramento da Usina Hidrelétrica de Ourinhos, para os períodos Pré e Pós enchimento. 


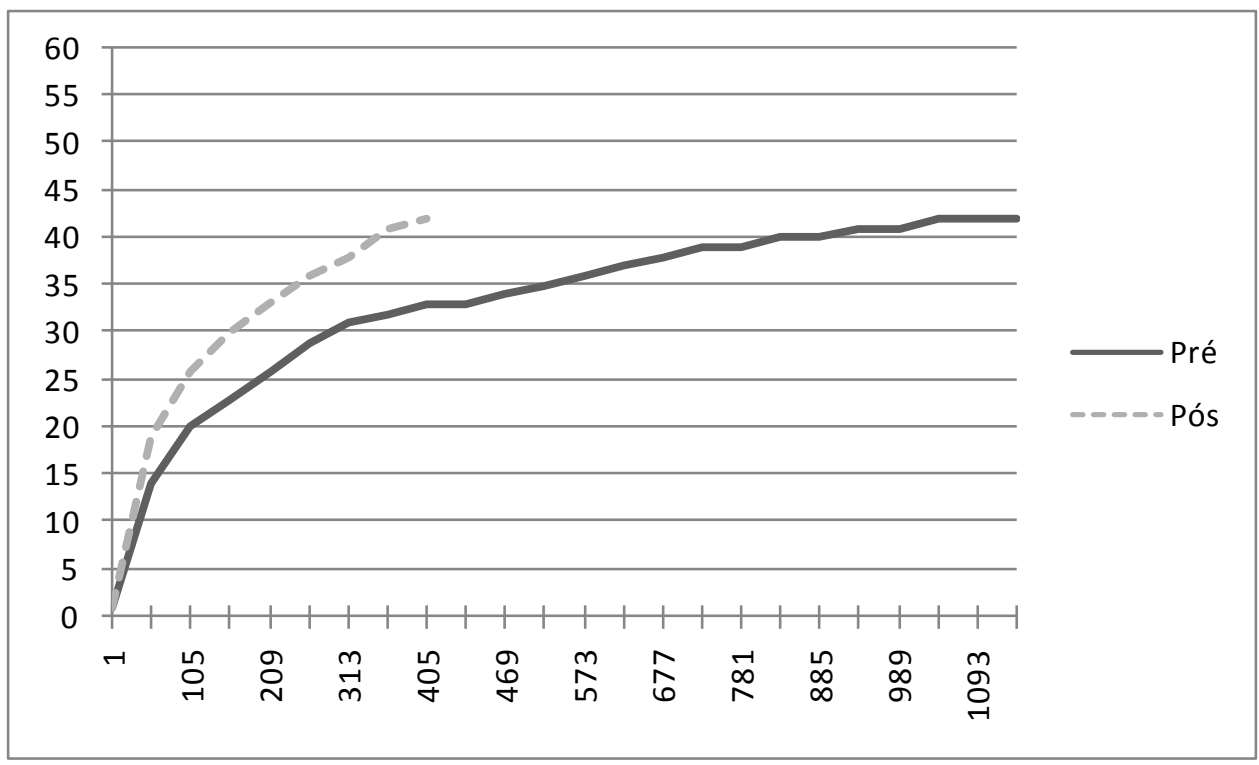

Figura 6. Curva de rarefação, segundo número de indivíduos coletados, para a estação de coleta Sítio Caiçara, no monitoramento da Usina Hidrelétrica de Ourinhos, para os períodos Pré e Pós enchimento.

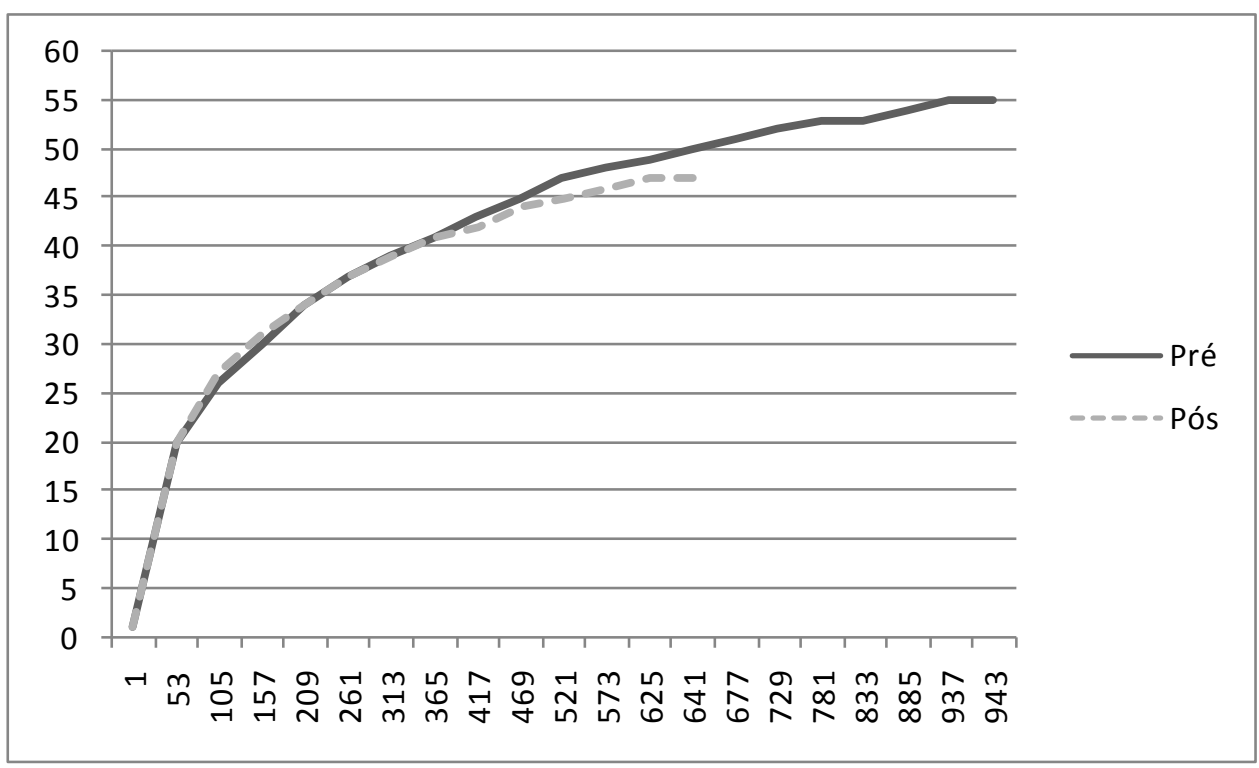

Figura 7. Curva de rarefação, segundo número de indivíduos coletados, para a estação de coleta Sítio Caiçara, no monitoramento da Usina Hidrelétrica de Ourinhos, para os períodos Pré e Pós enchimento. 


\subsection{Diversidade a}

Diversidade $\alpha$ é a diversidade local, correspondente ao número de espécies numa pequena área de habitat homogêneo. Esse tipo de diversidade é sensível a como se delimitam os habitats e quão intensamente se amostra uma comunidade (Whittaker,1972).

\subsection{1. Índice de Margalef}

A Tabela 2 e a Figura 8 mostram as diversidades calculadas pelo índice de Margalef para os períodos pré e pós enchimento das estações de coleta segundo o método de coleta, observa-se para o Canteiro de Obras uma redução da diversidade no período pós enchimento. Tanto Sítio Caiçara como Córrego Anhumas apresentaram aumento da diversidade no período pós para as coletas utilizando-se o aspirador elétrico. Porém quando analisamos o valor das três técnicas juntas (Geral) somente o Sítio Caiçara mostrou aumento.

Tabela 2. Índice de Margalef (D) dos períodos pré e pós enchimento, segundo método e estação de coleta, e geral.

\begin{tabular}{|l|r|r|r|r|r|r|r|r|}
\hline \multirow{2}{*}{ Ponto de Coleta } & \multicolumn{2}{|c|}{ Imaturos } & \multicolumn{1}{c|}{ Aspirador } & \multicolumn{2}{c|}{ Shannon } & \multicolumn{2}{c|}{ Geral } \\
\cline { 2 - 9 } & \multicolumn{1}{c|}{ Pré } & \multicolumn{1}{c|}{ Pós } & \multicolumn{1}{c|}{ Pré } & \multicolumn{1}{c|}{ Pós } & \multicolumn{1}{c|}{ Pré } & \multicolumn{1}{c|}{ Pós } & \multicolumn{1}{c|}{ Pré } & \multicolumn{1}{c|}{ Pós } \\
\hline Canteiro de Obras & 3,69 & 3,23 & 5,43 & 4,15 & 4,89 & 4,74 & 6,99 & 6,58 \\
\hline Sítio Caiçara & 2,47 & 3,52 & 3,34 & 3,61 & 3,97 & 3,75 & 5,85 & 6,83 \\
\hline Córrego Anhumas & 4,19 & 2,58 & 5,07 & 5,74 & 5,53 & 6,11 & 7,88 & 7,12 \\
\hline
\end{tabular}




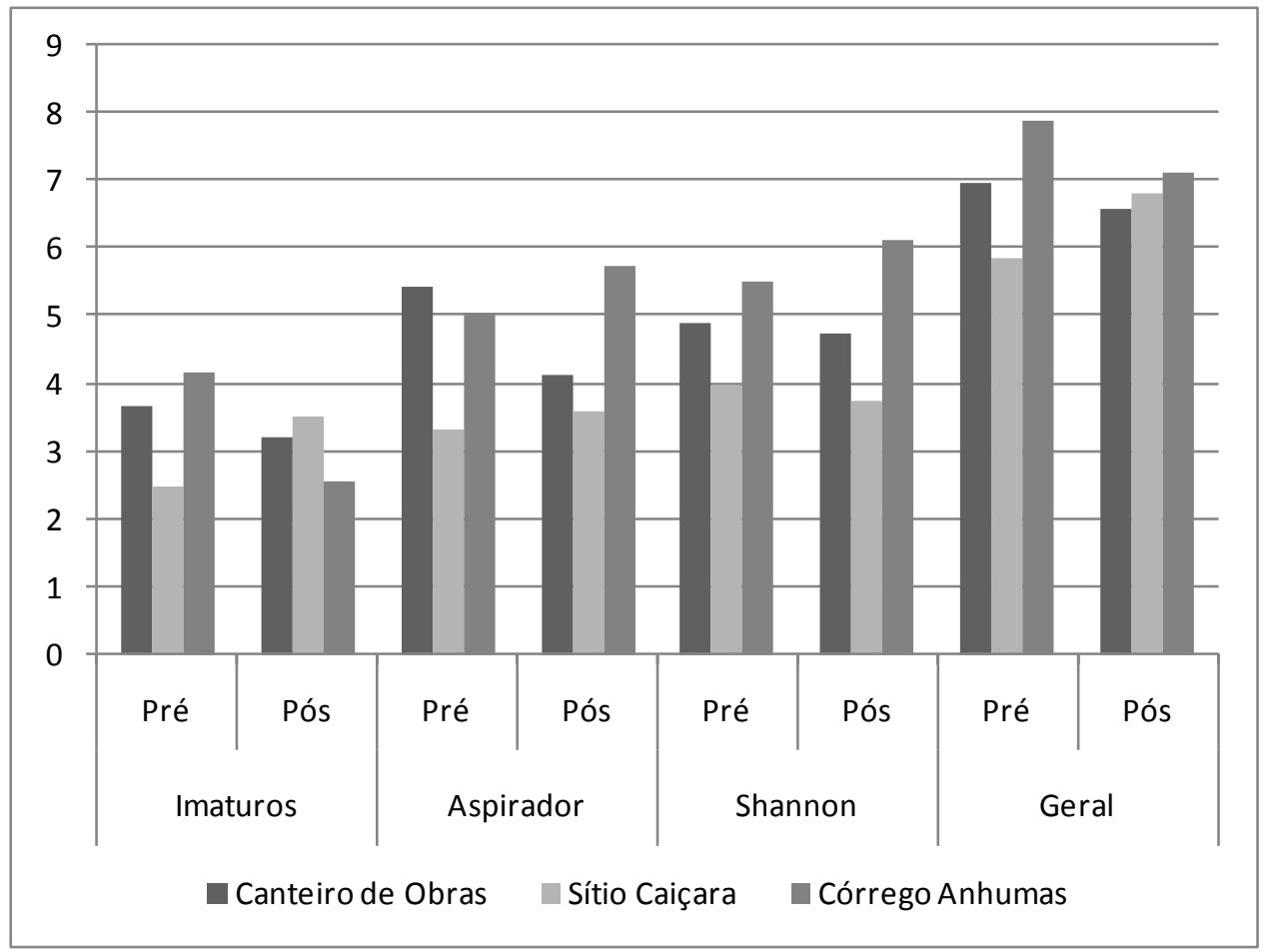

Figura 8. Índice de Magalef (D) dos períodos pré e pós enchimento, segundo método e estação de coleta, e geral.

\subsubsection{Dominância}

Os índices de dominância apresentados na Tabela 3 e ilustrados pela Figura 9 mostram-se maiores no período pós enchimento para o Canteiro de Obras nas três técnicas utilizadas, e para as coletas de formas imaturas do Córrego Anhumas. O Sítio Caiçara em contrapartida apresentou redução dos valores do índice de dominância. Quando utilizamos os dados obtidos pelas três técnicas para calcular a dominância, Canteiro de Obras e Córrego Anhumas apresentam alterações sutis, enquanto a da estação Sítio Caiçara sofreu forte redução. 
Tabela 3. Dominância (Índice de Simpson) dos períodos Pré e Pós enchimento, segundo método e estação de coleta, e geral.

\begin{tabular}{|l|c|c|r|r|r|r|r|r|}
\hline \multirow{2}{*}{ Ponto de Coleta } & \multicolumn{2}{|c|}{ Imaturos } & \multicolumn{2}{c|}{ Aspirador } & \multicolumn{2}{c|}{ Shannon } & \multicolumn{2}{c|}{ Geral } \\
\cline { 2 - 9 } & \multicolumn{1}{c|}{ Pré } & \multicolumn{1}{c|}{ Pós } & \multicolumn{1}{c|}{ Pré } & \multicolumn{1}{c|}{ Pós } & \multicolumn{1}{c|}{ Pré } & \multicolumn{1}{c|}{ Pós } & \multicolumn{1}{c|}{ Pré } & \multicolumn{1}{c|}{ Pós } \\
\hline Canteiro de Obras & 0,17 & 0,18 & 0,09 & 0,12 & 0,19 & 0,23 & 0,15 & 0,16 \\
\hline Sítio Caiçara & 0,16 & 0,14 & 0,14 & 0,13 & 0,35 & 0,28 & 0,18 & 0,08 \\
\hline Córrego Anhumas & 0,13 & 0,28 & 0,10 & 0,09 & 0,11 & 0,08 & 0,08 & 0,07 \\
\hline
\end{tabular}

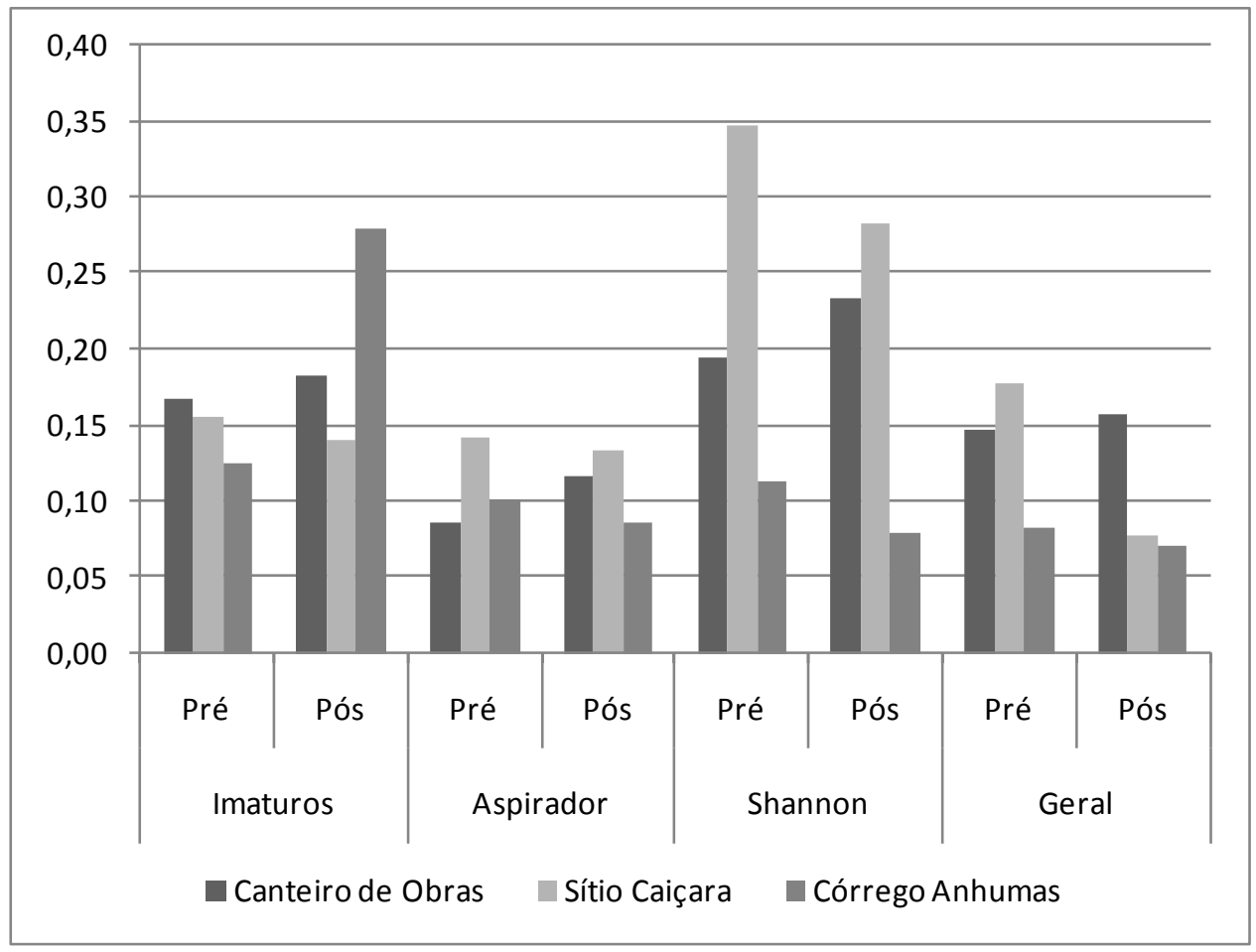

Figura 9. Dominância (Indíce de Simpson) dos períodos Pré e Pós enchimento, segundo método e estação de coleta, e geral.

\subsection{3. Índice de Abundância de Espécie Padronizado}

Os valores de Abundância, avaliados pelo Índice de Abundância de Espécie Padronizado (IAEP) obtidos na técnica de coleta com concha para pesquisa de formas imaturas, no Canteiro de Obras estão disponibilizados na Tabela 4 e ilustrados na Figura 10, a seguir apresentadas. 
Tabela 4. IAEP na estação de coleta Canteiro de Obras obtida nas coletas com concha entomológica para avaliação de formas imaturas, no monitoramento da Usina Hidrelétrica de Ourinhos, nos períodos pré e pós enchimento do reservatório.

\begin{tabular}{|l|l|r|}
\hline \multicolumn{1}{|c|}{ Pré } & Código & \multicolumn{1}{|c|}{ IAEP } \\
\hline Espécie & E39 & 0,45 \\
\hline Cx. (Cux.) grupo Coronator (Cux.) sp. & E44 & 0,36 \\
\hline Cx. (Cux.) coronator & E34 & 0,33 \\
\hline Ae. (Och.) fluviatilis & E4 & 0,26 \\
\hline An. (Nys.) albitarsis s.l & E11 & 0,17 \\
\hline Cx. (Cux.) quinquefascistus & E43 & 0,14 \\
\hline An. (Nys.) galvaoi & E14 & 0,13 \\
\hline Cx. (Cux.) declarator & E35 & 0,13 \\
\hline Cx. (Cux.) mollis & E41 & 0,13 \\
\hline Cx. (Mel.) ribeirensis & E58 & 0,13 \\
\hline Ur. (Ura.) geometrica & E86 & 0,13 \\
\hline Ae. (Och.) scapularis & E6 & 0,12 \\
\hline Ps. ciliata & E75 & 0,12 \\
\hline An. (Nys.) sp & E15 & 0,08 \\
\hline An. (Nys.) strodei/ rondoni & E17 & 0,08 \\
\hline Ur. (Ura.) nataliae & E88 & 0,08 \\
\hline Wy. sp. & E92 & 0,08 \\
\hline An. (Nys.) argyritarsis & E19 & 0,07 \\
\hline Ps. confinnis & E77 & 0,07 \\
\hline Ps. ferox & E79 & 0,05 \\
\hline Cx. (Cux.) chidesteri & E33 & 0,04 \\
\hline Ae. (Stg.) albopictus & E2 & 0,02 \\
\hline
\end{tabular}

Pós

\begin{tabular}{|l|l|r|}
\hline Espécie & Código & \multicolumn{1}{|c|}{ IAEP } \\
\hline Cx. (Cux.) grupo Coronator & E39 & 0,78 \\
\hline Cx. (Cux.) coronator & E34 & 0,56 \\
\hline An. (Nys.) argyritarsis & E19 & 0,26 \\
\hline An. (Nys.) albitarsis s.l & E11 & 0,24 \\
\hline Ur. (Ura.) geometrica & E86 & 0,21 \\
\hline Ae. (Och.) fluviatilis & E4 & 0,20 \\
\hline Cx. (Cux.) quinquefascistus & E43 & 0,18 \\
\hline Wy. rooti & E91 & 0,18 \\
\hline Wy. sp. & E92 & 0,18 \\
\hline Cx. (Cux.) sp. & E44 & 0,15 \\
\hline Cx. (Mcx.) imitator & E45 & 0,15 \\
\hline Ae. (Stg.) albopictus & E2 & 0,13 \\
\hline Ae. (Och.) scapularis & E6 & 0,13 \\
\hline Cx. (Cux.) dolosus & E36 & 0,13 \\
\hline Cx. (Cux.) declarator & E35 & 0,10 \\
\hline Ur. (Ura.) lowii & E83 & 0,10 \\
\hline Cx. (Mel.) seção Melanoconion & E59 & 0,08 \\
\hline Ur. (Ura.) pulcherrima & E84 & 0,05 \\
\hline An. (Nys.) sp & E15 & 0,02 \\
\hline
\end{tabular}



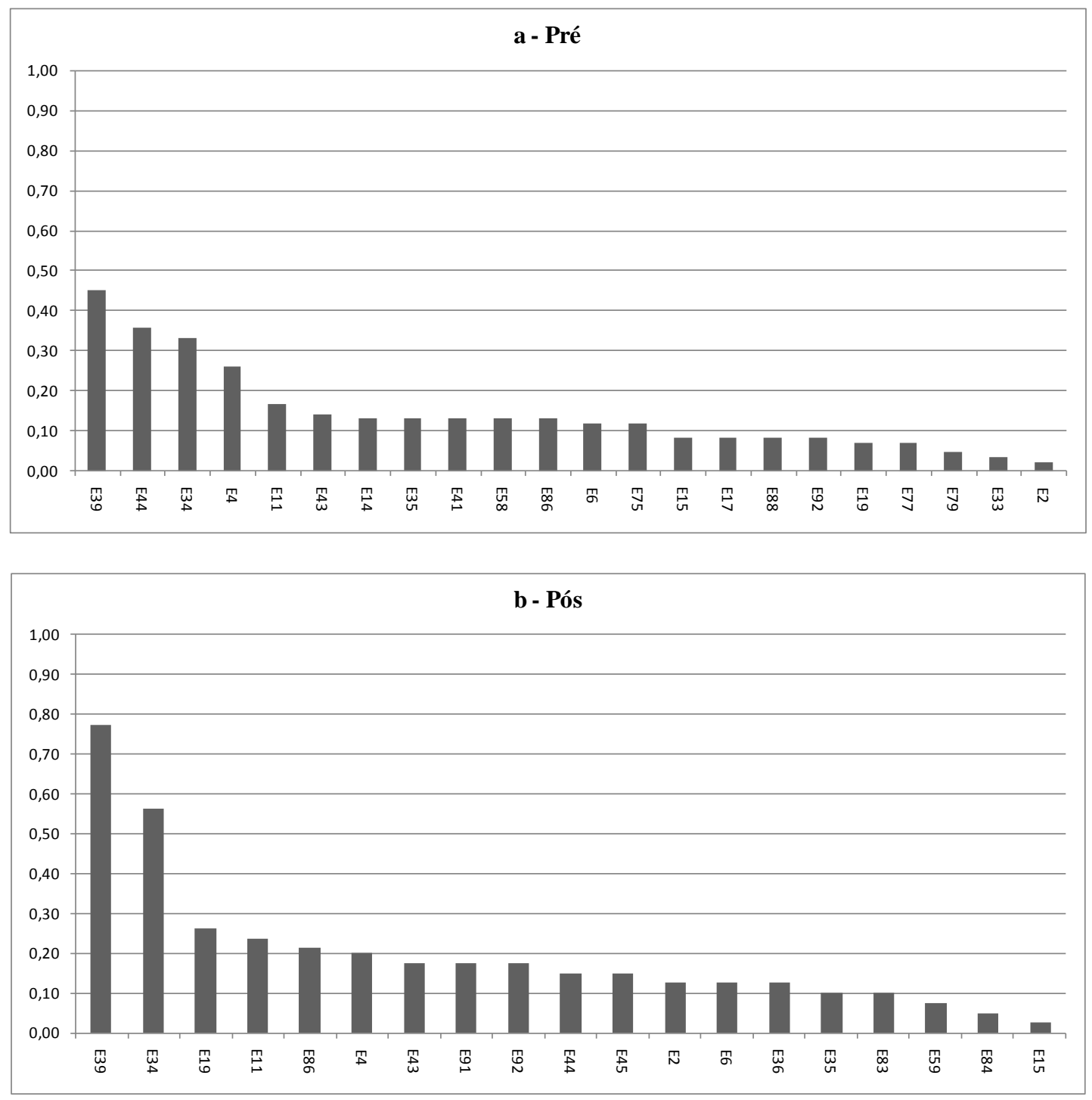

Figura 10. IAEP na estação de coleta Canteiro de Obras obtida nas coletas com concha entomológica para avaliação de formas imaturas, no monitoramento da Usina Hidrelétrica de Ourinhos, nos períodos pré (a) e pós (b) enchimento do reservatório.

Observou-se uma discreta redução do número de espécies no período pós enchimento do reservatório. Culex (Culex) grupo Coronator manteve-se como primeiro no "ranking” de abundância apresentando um aumento no valor de IAEP. O mosquito Culex (Culex) declarator caiu da $8^{\mathrm{a}}$ posição para a $15^{\mathrm{a}}$, enquanto o Aedes albopictus, mosquito raro no período pré enchimento, subiu da $22^{\mathrm{a}}$ para $12^{\mathrm{a}}$ posição. 
Tabela 5. IAEP na estação de coleta Sítio Caiçara obtida nas coletas com concha entomológica para avaliação de formas imaturas, no monitoramento da Usina Hidrelétrica de Ourinhos, nos períodos pré e pós enchimento do reservatório.

\begin{tabular}{|c|c|c|c|c|c|}
\hline \multicolumn{3}{|c|}{ Pré } & \multicolumn{3}{|l|}{ Pós } \\
\hline Espécie & Código & IAEP & Espécie & Código & IAEP \\
\hline Cx. (Cux.) grupo Coronator & E39 & 0,64 & An. (Nys.) albitarsis s.l & E11 & 0,67 \\
\hline Cx. (Cux.) coronator & E34 & 0,60 & Ad. (Ady.) squamipennis & E1 & 0,47 \\
\hline Cx. (Cux.) sp. & E44 & 0,38 & An. (Nys.) argyritarsis & E19 & 0,45 \\
\hline Ae. (Och.) scapularis & E6 & 0,32 & Cx. (Cux.) grupo Coronator & E39 & 0,44 \\
\hline CX. (Cux.) dolosus & E36 & 0,30 & Cx. (Cux.) coronator & E34 & 0,31 \\
\hline Ae.(Och.) terrens & E9 & 0,26 & Cx. (Cux.) mollis & E41 & 0,29 \\
\hline Ae. (Stg.) albopictus & E2 & 0,21 & An. (Nys.) sp & E15 & 0,27 \\
\hline An. (Nys.) strodei/ rondoni & E17 & 0,20 & An. (Nys.) strodei & E16 & 0,26 \\
\hline Cx. (Cux.) mollis & E41 & 0,20 & Ae. (Och.) fluviatilis & E3 & 0,20 \\
\hline Cx. (Cux.) declarator & E35 & 0,17 & Cx. (Cux.) chidesteri & E33 & 0,20 \\
\hline An. (Nys.) albitarsis s.l & E11 & 0,11 & Cx. (Mel.) sp & E60 & 0,18 \\
\hline Cx. (Cux.) chidesteri & E33 & 0,10 & An. (Nys.) triannulatus & E18 & 0,15 \\
\hline Cx. (Cux.) dolosus/eduardoi & E37 & 0,09 & Cx. (Cux.) sp. & E44 & 0,15 \\
\hline An. (Nys.) argyritarsis & E19 & 0,07 & Cx. (Mel.) aureonotatus & E46 & 0,15 \\
\hline \multirow[t]{6}{*}{ Cx. (Cux.) eduardoi } & E38 & 0,06 & Cx. (Mel.) vaxus & E62 & 0,14 \\
\hline & & & Ur. (Ura.) lowii & E83 & 0,14 \\
\hline & & & An. (Nys.) darlingi & E13 & 0,12 \\
\hline & & & Cx. (Mel.) seção Melanoconion & E59 & 0,09 \\
\hline & & & Cx. (Mel.) dureti & E50 & 0,06 \\
\hline & & & Ur. (Ura.) sp. & E85 & 0,02 \\
\hline
\end{tabular}

O número de espécies apresentou um aumento acentuado na segunda fase do estudo, como se pode observar na Tabela 5, que exibe o IAEP obtido na técnica de coleta com concha para a pesquisa de formas imaturas no Sítio Caiçara.

A Figura 11 ilustra as variações de posição das espécies entre os períodos pré e pós enchimento. 

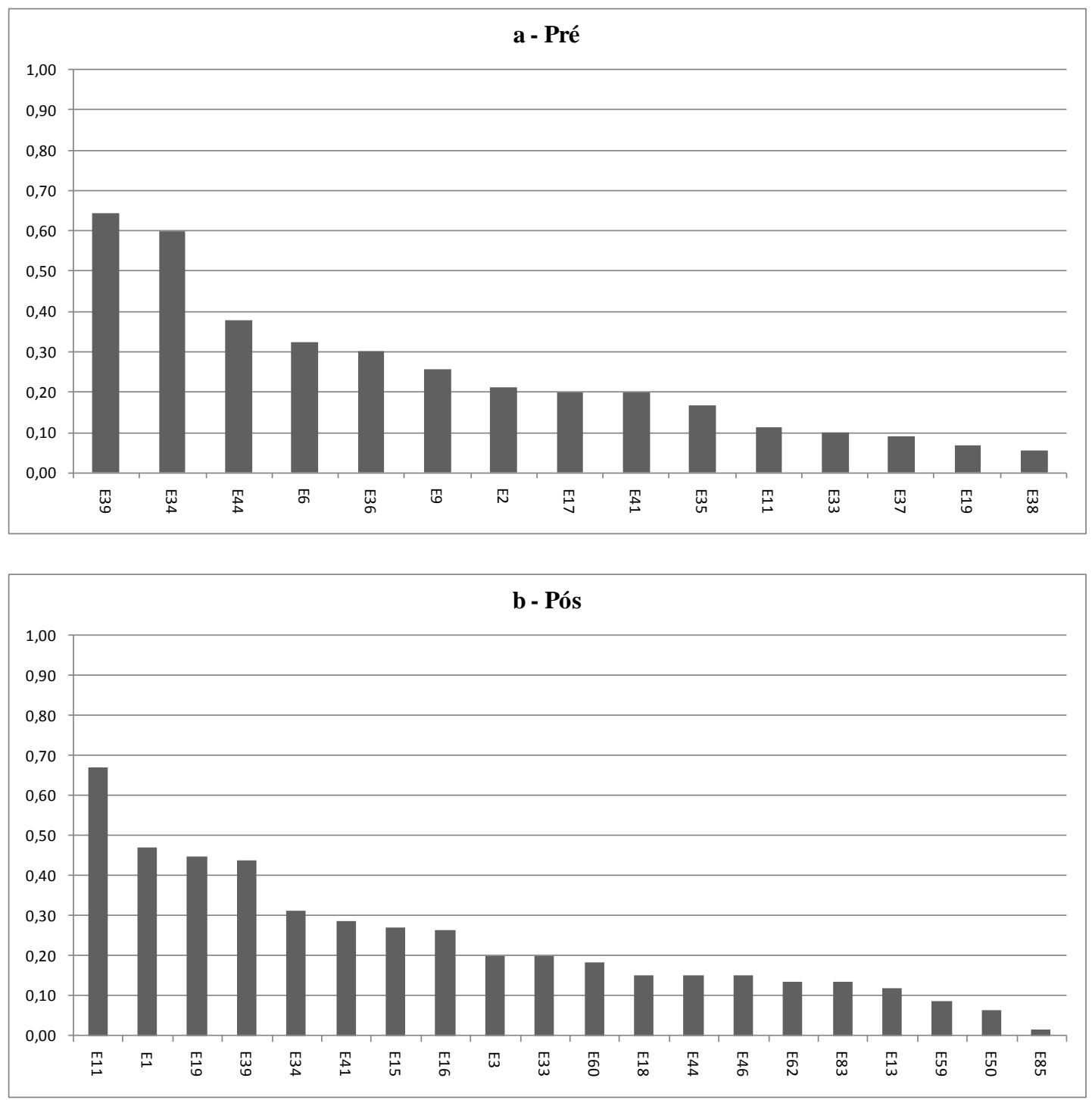

Figura 11. IAEP na estação de coleta Sítio Caiçara obtida nas coletas com concha entomológica para avaliação de formas imaturas, no monitoramento da Usina Hidrelétrica de Ourinhos, nos períodos pré (a) e pós (b) enchimento do reservatório

O Anopheles albitarsis s.l subiu da $11^{\mathrm{a}}$ para a $1^{\mathrm{a}}$ posição no período pós enchimento, enquanto Aedes scapularis que apareceu na $4^{\mathrm{a}}$ posição no período pré enchimento não foi coletado no período pós. Anopheles triannulatus e Anopheles (Nys.) darlingi foram capturados somente na segunda fase do estudo e ocupando a $12^{\mathrm{a}}$ e $18^{\mathrm{a}}$ posições de abundância, respectivamente. 
Observa-se que houve uma redução na fauna de culicídeos no segundo período do estudo (Tab. 6 e Fig. 12). Destaca-se a presença de Aedes scapularis ocupando a $3^{\mathrm{a}}$ posição na primeira fase e seu desaparecimento no período seguinte. $\mathrm{O}$ Anopheles argyritarsis caiu da $2^{\mathrm{a}}$ para a $12^{\mathrm{a}}$ posição. Enquanto Anopheles albitarsis s.l passou da $6^{\mathrm{a}}$ para a $2^{\mathrm{a}}$ posição. Outra espécie que merece destaque é Culex quinquefasciatus que só foi registrada no período pós enchimento.

Tabela 6. IAEP na estação de coleta Córrego Anhumas obtida nas coletas com concha entomológica para avaliação de formas imaturas, no monitoramento da Usina Hidrelétrica de Ourinhos, nos períodos pré e pós enchimento do reservatório.

\begin{tabular}{|c|c|c|c|c|c|}
\hline \multicolumn{3}{|l|}{ Pré } & \multicolumn{3}{|l|}{ Pós } \\
\hline Espécie & Código & IAEP & Espécie & Código & IAEP \\
\hline Ad. (Ady.) squamipennis & E1 & 0,48 & Ad. (Ady.) squamipennis & E1 & 0,91 \\
\hline An. (Nys.) argyritarsis & E19 & 0,46 & An. (Nys.) albitarsis s.l & E11 & 0,88 \\
\hline Ae. (Och.) scapularis & E6 & 0,36 & Cx. (Cux.) grupo Coronator & E39 & 0,35 \\
\hline Cx. (Cux.) coronator & E34 & 0,34 & Cx. (Mel.) seção Melanoconion & E59 & 0,26 \\
\hline Cx. (Cux.) grupo Coronator & E39 & 0,34 & Cx. (Cux.) sp. & E44 & 0,21 \\
\hline An. (Nys.) albitarsis s.l & E11 & 0,29 & Ur. pulcherrima & E83 & 0,18 \\
\hline Ur. pulcherrima & E83 & 0,18 & Cx. (Cux.) quinquefascistus & E43 & 0,15 \\
\hline Wy. (Wye.) medioalbipes & E90 & 0,17 & Cx. (Mel.) aureonotatus & E46 & 0,15 \\
\hline Cx. (Cux.) chidesteri & E33 & 0,15 & Cx. (Cux.) coronator & E34 & 0,13 \\
\hline Cx. (Mel.) vaxus & E62 & 0,15 & An. (Nys.) sp & E15 & 0,09 \\
\hline Ur. (Ura.) lowii & E82 & 0,15 & Cx. (Mel.) vaxus & E62 & 0,09 \\
\hline An. (Nys.) sp & E15 & 0,14 & An. (Nys.) argyritarsis & E19 & 0,08 \\
\hline Cx. (Mel.) bastagarius & E47 & 0,11 & An. (Nys.) strodei & E16 & 0,06 \\
\hline Cx. (Mel.) sp. & E60 & 0,11 & Cx.(Cux.) bidens & E32 & 0,02 \\
\hline Ur. (Ura.) geometrica & E86 & 0,09 & Ur. sp & E89 & 0,02 \\
\hline Cx. (Mcx.) imitator & E45 & 0,08 & & & \\
\hline Cx. (Mel.) seção Melanoconion & E59 & 0,08 & & & \\
\hline Ps. sp. & E80 & 0,08 & & & \\
\hline Cx. (Cux.) sp. & E44 & 0,06 & & & \\
\hline Ps. cingulata & E76 & 0,04 & & & \\
\hline Ae. (Och.) serratus & E7 & 0,03 & & & \\
\hline An. (Nys.) galvaoi & E14 & 0,03 & & & \\
\hline Cx. (Cux.) dolosus & E36 & 0,03 & & & \\
\hline Ps. confinnis & E77 & 0,03 & & & \\
\hline
\end{tabular}



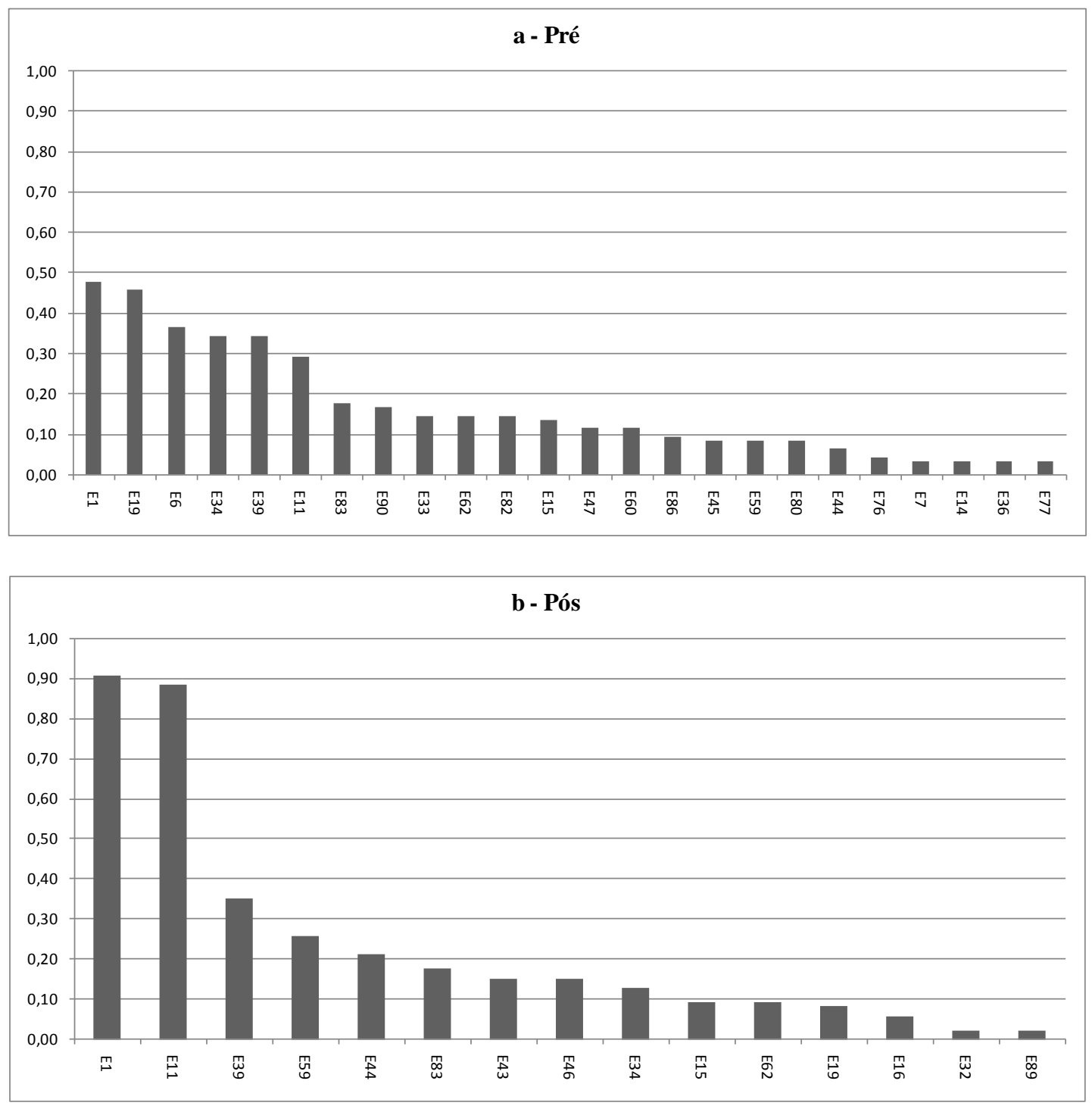

Figura 12. IAEP na estação de coleta Córrego Anhumas obtida nas coletas com concha entomológica para avaliação de formas imaturas, no monitoramento da Usina Hidrelétrica de Ourinhos, nos períodos pré (a) e pós (b) enchimento do reservatório.

A Tabela 7 e a Figura 13 apresentam os valores do IAEP obtidos na técnica de coleta com aspirador elétrico no Canteiro de Obras.

Observa-se na Figura 13, marcante redução no número de espécies na segunda fase relativa ao período pós impacto. Culex (Culex) sp. foi a “espécie” mais abundante nas duas fases de estudo, Culex (Culex) dolosus e Culex (Culex) 
declarator apresentaram movimentos opostos entre o período pré e pós enchimento, o primeiro subindo da $14^{\mathrm{a}}$ para a $2^{\mathrm{a}}$ posição e o segundo caindo da $3^{\mathrm{a}}$ para a $10^{\mathrm{a}}$. $\mathrm{O}$ mosquito Aedes scapularis permaneceu na sexta posição em ambas as fases. Duas espécies epidemiologicamente importantes foram capturadas apenas na segunda fase do estudo: Culex quinquefasciatus e Aedes albopictus, porém, foram raras.

Tabela 7. IAEP na estação de coleta Canteiro de Obras obtida nas coletas com aspirador elétrico, no monitoramento da Usina Hidrelétrica de Ourinhos, nos períodos pré e pós enchimento do reservatório.

\begin{tabular}{|l|l|c|}
\hline \multicolumn{1}{|c|}{ Pré } \\
\hline Espécie & Código & IAEP \\
\hline Cx. (Cux.) sp. & E44 & 0,42 \\
\hline Ur. (Ura.) mathesoni & E87 & 0,37 \\
\hline Cx. (Cux.) declarator & E35 & 0,24 \\
\hline Wy. sp. & E92 & 0,24 \\
\hline Ur. (Ura.) pulcherrima & E84 & 0,22 \\
\hline Ae. (Och.) scapularis & E6 & 0,17 \\
\hline Cq. (Rhy.) albicosta & E23 & 0,17 \\
\hline Cx. (Mel.) seção Melanoconion & E59 & 0,14 \\
\hline Ur. (Ura.) geometrica & E86 & 0,14 \\
\hline Cx. (Mel.) ribeirensis & E58 & 0,13 \\
\hline Cq. (Rhy.) chrysonotum & E24 & 0,12 \\
\hline Cx. (Cux.) dolosus/eduardoi & E37 & 0,12 \\
\hline Cx. (Cux.) nigripalpus & E42 & 0,10 \\
\hline Cx. (Cux.) dolosus & E36 & 0,09 \\
\hline Ps. (Jan.) albigenu & E73 & 0,09 \\
\hline Cx. (Mel.) plectoporpe & E56 & 0,06 \\
\hline Ur. (Ura.) nataliae & E88 & 0,06 \\
\hline Cq. (Rhy.) hermanoi & E26 & 0,03 \\
\hline Cq. (Rhy.) venezuelensis & E30 & 0,03 \\
\hline Cx. (Ads.) amazonenzis & E31 & 0,03 \\
\hline Cx. (Cux.) coronator & E34 & 0,03 \\
\hline Cx. (Cux.) grupo Coronator & E39 & 0,03 \\
\hline Cx. (Mel.) ocossa & E54 & 0,03 \\
\hline Ps. (Jan.) lutzii & E80 & 0,03 \\
\hline
\end{tabular}

\begin{tabular}{|l|l|r|}
\hline \multicolumn{1}{|c}{ Pós } & Código & \multicolumn{1}{|c|}{ IAEP } \\
\hline Espécie & $\mathrm{E} 44$ & 0,55 \\
\hline Cx. (Cux.) sp. & $\mathrm{E} 36$ & 0,43 \\
\hline Cx. (Cux.) dolosus & $\mathrm{E} 87$ & 0,32 \\
\hline Ur. (Ura.) mathesoni & $\mathrm{E} 30$ & 0,20 \\
\hline Cq. (Rhy.) venezuelensis & $\mathrm{E} 52$ & 0,13 \\
\hline Cx. (Mel.) grupo Atratus & $\mathrm{E} 6$ & 0,12 \\
\hline Ae. (Och.) scapularis & $\mathrm{E} 24$ & 0,12 \\
\hline Cq. (Rhy.) chrysonotum & $\mathrm{E} 88$ & 0,12 \\
\hline Ur. (Ura.) nataliae & $\mathrm{E} 2$ & 0,08 \\
\hline Ae. (Stg.) albopictus & $\mathrm{E} 35$ & 0,08 \\
\hline Cx. (Cux.) declarator & $\mathrm{E} 43$ & 0,08 \\
\hline Cx. (Cux.) quinquefascistus & 0,08 \\
\hline Cx. (Mel.) rabelloi & $\mathrm{E} 57$ & 0,08 \\
\hline Cx. (Mel.) sphatulatus & $\mathrm{E} 61$ & 0,03 \\
\hline Cx. (Cux.) bidens & $\mathrm{E} 32$ & 0,03 \\
\hline Ps. (Jan.) ferox & $\mathrm{E} 79$ & 0,03 \\
\hline Ur. (Ura.) pulcherrima & $\mathrm{E} 84$ & \\
\hline & & \\
\hline & & \\
\hline & & \\
\hline & & \\
\hline & & \\
\hline
\end{tabular}



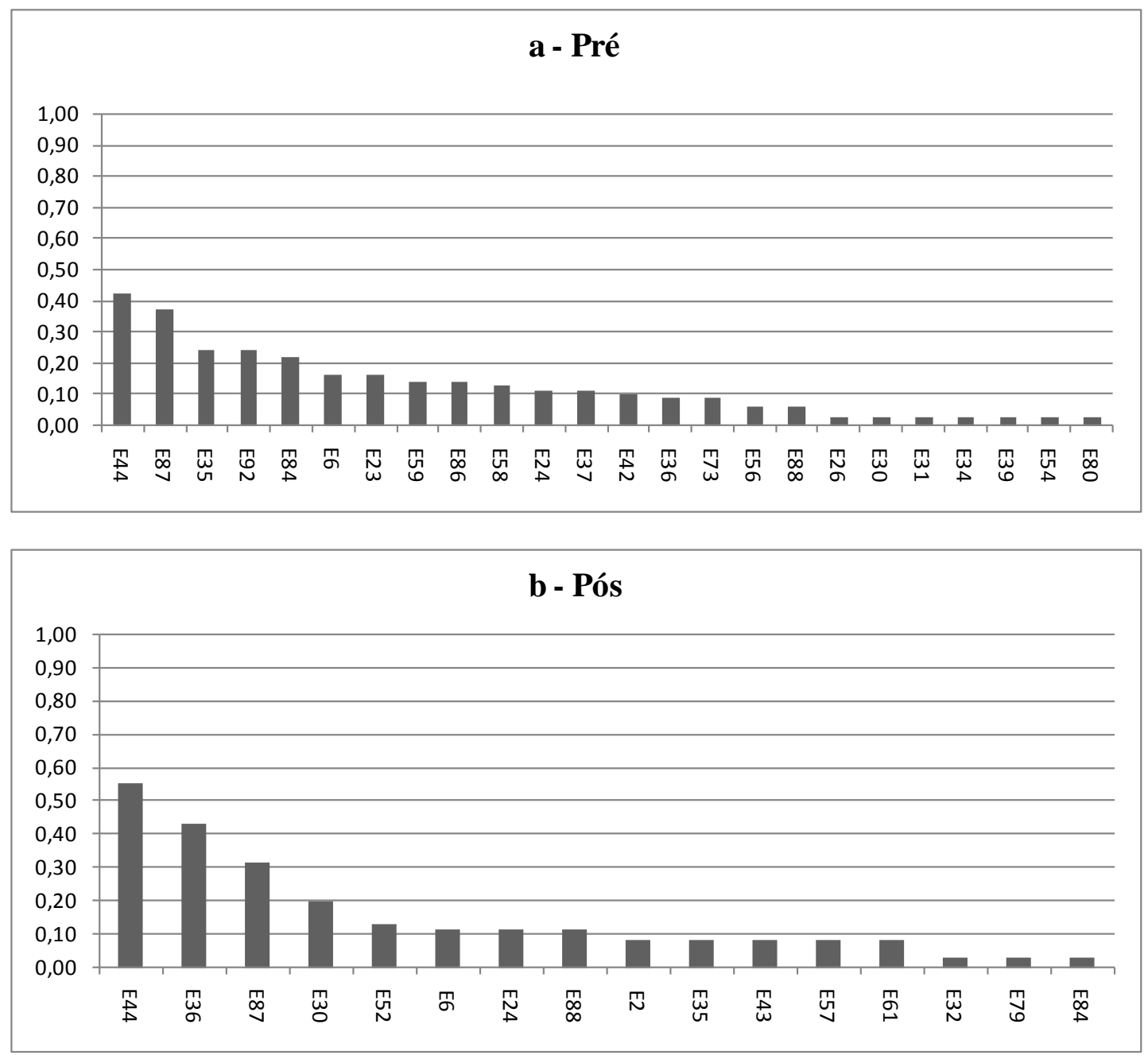

Figura 13. IAEP na estação de coleta Canteiro de Obras obtida nas coletas com aspirador elétrico, no monitoramento da Usina Hidrelétrica de Ourinhos, nos períodos pré (a) e pós (b) enchimento do reservatório.

Os valores avaliados pelo IAEP obtidos no Sítio Caiçara pela técnica de coleta com aspirador elétrico estão disponibilizados na Tabela 8 e ilustrados na Figura 14.

Não houve redução de espécies na segunda fase do estudo. Aedes scapularis ocupou a posição de maior abundância antes da inundação, passando para a $7^{\mathrm{a}}$. posição no período pós fechamento da barragem. O mosquito Aedes albopictus foi coletado apenas na primeira fase do estudo. Anopheles (Nys.) darlingi não foi 
coletado na primeira fase, porém, na fase pós-inundação ocupa posição de destaque, ou seja, a $5^{\mathrm{a}}$ posição.

Tabela 8. IAEP na estação de coleta Sítio Caiçara obtida nas coletas com aspirador elétrico, no monitoramento da Usina Hidrelétrica de Ourinhos, nos períodos pré e pós enchimento do reservatório

\begin{tabular}{|l|l|c|}
\hline \multicolumn{1}{|c|}{ Pré } \\
\hline Espécie & Código & IAEP \\
\hline Ae. (Och.) scapularis & E6 & 0,63 \\
\hline Cx. (Cux.) declarator & E35 & 0,63 \\
\hline Cx. (Cux.) dolosus & E36 & 0,60 \\
\hline Cx. (Cux.) sp. & E44 & 0,41 \\
\hline Ae. (Stg.) albopictus & E2 & 0,35 \\
\hline Cx. (Cux.) grupo Coronator & E39 & 0,27 \\
\hline Cx. (Cux.) dolosus/eduardoi & E37 & 0,16 \\
\hline Cx. (Cux.) lygrus & E40 & 0,16 \\
\hline Cx. (Cux.) quinquefascistus & E43 & 0,15 \\
\hline Cx. (Cux.) coronator & E34 & 0,11 \\
\hline Cx. (Cux.) mollis & E41 & 0,10 \\
\hline Wy. sp. & E92 & 0,10 \\
\hline Cx. (Cux.) chidesteri & E33 & 0,09 \\
\hline Cx. (Mel.) pilosus & E55 & 0,08 \\
\hline Cq. (Rhy.) venezuelensis & E30 & 0,07 \\
\hline Cx. (Mel.) ocossa & E54 & 0,04 \\
\hline Ur. (Ura.) pulcherrima & E84 & 0,02 \\
\hline
\end{tabular}

\begin{tabular}{|l|l|r|}
\hline Pós & Código & \multicolumn{1}{|c|}{ IAEP } \\
\hline Expécie $($ Cux.) sp. & $\mathrm{E} 44$ & 0,60 \\
\hline Cq. (Rhy.) venezuelensis & $\mathrm{E} 30$ & 0,57 \\
\hline Cx. (Cux.) dolosus/eduardoi & $\mathrm{E} 37$ & 0,19 \\
\hline Ae. (Och.) crinifer & $\mathrm{E} 3$ & 0,18 \\
\hline An. darlingi & $\mathrm{E} 13$ & 0,18 \\
\hline Cx. (Cux.) chidesteri & $\mathrm{E} 33$ & 0,18 \\
\hline Ae. (Och.) scapularis & $\mathrm{E} 6$ & 0,16 \\
\hline Ad. (Ady.) squamipennis & $\mathrm{E} 1$ & 0,14 \\
\hline Cx. (Cux.) dolosus & $\mathrm{E} 36$ & 0,14 \\
\hline Ae. (Och.) serratus/nubilus & $\mathrm{E} 8$ & 0,10 \\
\hline Cx. (Cux.) coronator & $\mathrm{E} 34$ & 0,10 \\
\hline Wy. sp. & $\mathrm{E} 92$ & 0,10 \\
\hline Ae. (Och.) nubilus & $\mathrm{E} 5$ & 0,02 \\
\hline Ae. (Och.) serratus & $\mathrm{E} 7$ & 0,02 \\
\hline Cq. (Rhy.) juxtamansonia & $\mathrm{E} 27$ & 0,02 \\
\hline Cx. (Cux.) lygrus & $\mathrm{E} 40$ & 0,02 \\
\hline & & \\
\hline
\end{tabular}



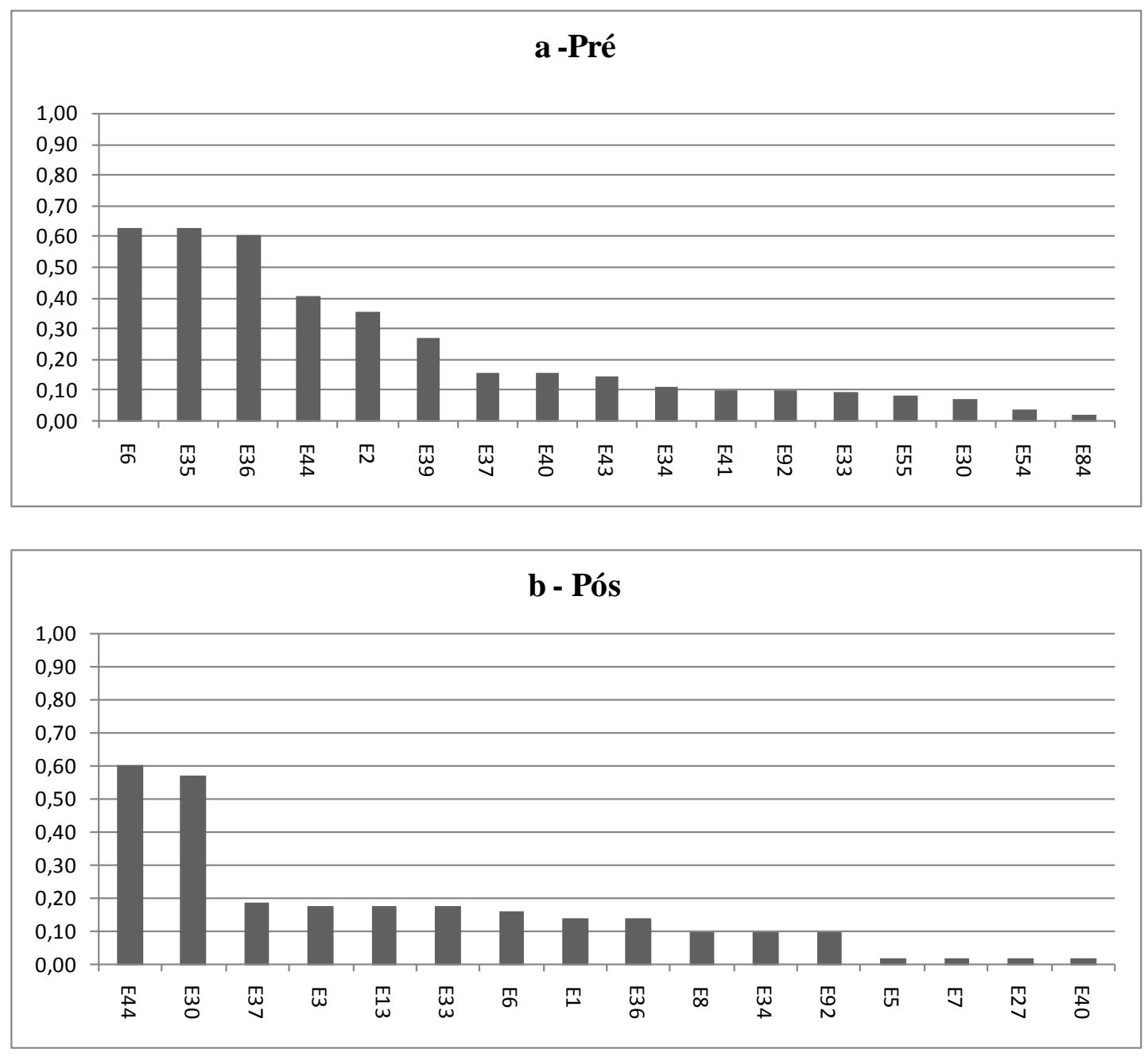

Figura 14. IAEP na estação de coleta Sítio Caiçara obtida nas coletas com aspirador elétrico, no monitoramento da Usina Hidrelétrica de Ourinhos, nos períodos pré (a) e pós (b) enchimento do reservatório.

A fauna de culicídeos pouco se modificou com a inundação, mantendo o número de espécie aproximado (Tab. 9 e Fig. 15). O mosquito Aedes scapularis caiu da $3^{\mathrm{a}}$. para a $4^{\mathrm{a}}$. posição na abundância após a inundação. O Anopheles albitarsis s.l, mais raro na primeira fase, subiu da $23^{\mathrm{a}}$. para a $15^{\mathrm{a}}$. posição. O principal vetor da malária, o Anopheles (Nys.) darlingi foi capturado somente na segunda fase do estudo e ocupando a 9a . posição do índice de abundância. 
Tabela 9. IAEP na estação de coleta Córrego Anhumas obtida nas coletas com aspirador elétrico, no monitoramento da Usina Hidrelétrica de Ourinhos, nos períodos pré e pós enchimento do reservatório.

\begin{tabular}{|l|l|c|}
\hline \multicolumn{1}{|l}{ Pré } & Código & IAEP \\
\hline Espécie & E5 & 0,83 \\
\hline Ae. (Och.) nubilus & E35 & 0,76 \\
\hline Cx. (Cux.) declarator & E6 & 0,49 \\
\hline Ae. (Och.) scapularis & E34 & 0,49 \\
\hline Cx. (Cux.) coronator & E7 & 0,33 \\
\hline Ae. (Och.) serratus & E44 & 0,31 \\
\hline Cx. (Cux.) sp. & E33 & 0,25 \\
\hline Cx. (Cux.) chidesteri & E39 & 0,15 \\
\hline Cx. (Cux.) grupo Coronator & E1 & 0,13 \\
\hline Ad. (Ady.) squamipennis & E3 & 0,13 \\
\hline Ae. (Och.) crinifer & E30 & 0,13 \\
\hline Cq. (Rhy.) venezuelensis & E36 & 0,13 \\
\hline Cx. (Cux.) dolosus & E75 & 0,13 \\
\hline Ps. (Pso.) ciliata & E58 & 0,12 \\
\hline Cx. (Mel.) ribeirensis & E27 & 0,11 \\
\hline Cq. (Rhy.) juxtamansonia & E41 & 0,11 \\
\hline Cx. (Cux.) mollis & E66 & 0,11 \\
\hline Li. durhami & E32 & 0,10 \\
\hline Cx. (Cux.) bidens & E14 & 0,06 \\
\hline An. (Nys.) galvaoi & E34 & 0,05 \\
\hline Cx. (Cux.) saltanensis & E40 & 0,03 \\
\hline Cx. (Cux.) lygrus & E79 & 0,03 \\
\hline Ps. (Jan.) ferox & E12 & 0,01 \\
\hline An. (Nys.) albitarsis s.l & E52 & 0,01 \\
\hline Cx. (Mel.) grupo Atratus & E70 & 0,01 \\
\hline Ma. (Man.) indubitans & E86 & 0,01 \\
\hline Ur. (Ura.) geometrica & \\
\hline
\end{tabular}

\begin{tabular}{|l|l|r|}
\hline Espécie & Código & \multicolumn{1}{|c|}{ IAEP } \\
\hline Cx. (Cux.) sp. & E44 & 0,80 \\
\hline Cx. (Cux.) declarator & E35 & 0,41 \\
\hline Cx. (Cux.) grupo Coronator & E39 & 0,36 \\
\hline Ae. (Och.) scapularis & E6 & 0,26 \\
\hline Cx. (Cux.) bidens & E32 & 0,20 \\
\hline Ma. (Man.) flaveola & E68 & 0,20 \\
\hline Ur. (Ura.) pulcherrima & E84 & 0,19 \\
\hline Ad. (Ady.) squamipennis & E1 & 0,16 \\
\hline An. darlingi & E13 & 0,16 \\
\hline Ae. (Och.) crinifer & E3 & 0,14 \\
\hline Cx. (Cux.) coronator & E34 & 0,10 \\
\hline Cx. (Cux.) dolosus & E36 & 0,10 \\
\hline Ma. (Man.) titillans & E70 & 0,10 \\
\hline Ma. (Man.) wilsoni & E72 & 0,10 \\
\hline An. (Nys.) albitarsis s.l & E11 & 0,09 \\
\hline Cx. (Cux.) chidesteri & E33 & 0,09 \\
\hline Ps. (Jan.) ferox & E79 & 0,06 \\
\hline An. (Nys.) evansae & E20 & 0,03 \\
\hline An. (Nys.) galvaoi & E14 & 0,03 \\
\hline An. strodei & E16 & 0,03 \\
\hline An. triannulatus & E18 & 0,03 \\
\hline Cq. (Rhy.) venezuelensis & E30 & 0,03 \\
\hline Cx. (Mel.) ocossa & E54 & 0,03 \\
\hline Cx. (Mel.) zeteki & E63 & 0,03 \\
\hline & & \\
\hline
\end{tabular}



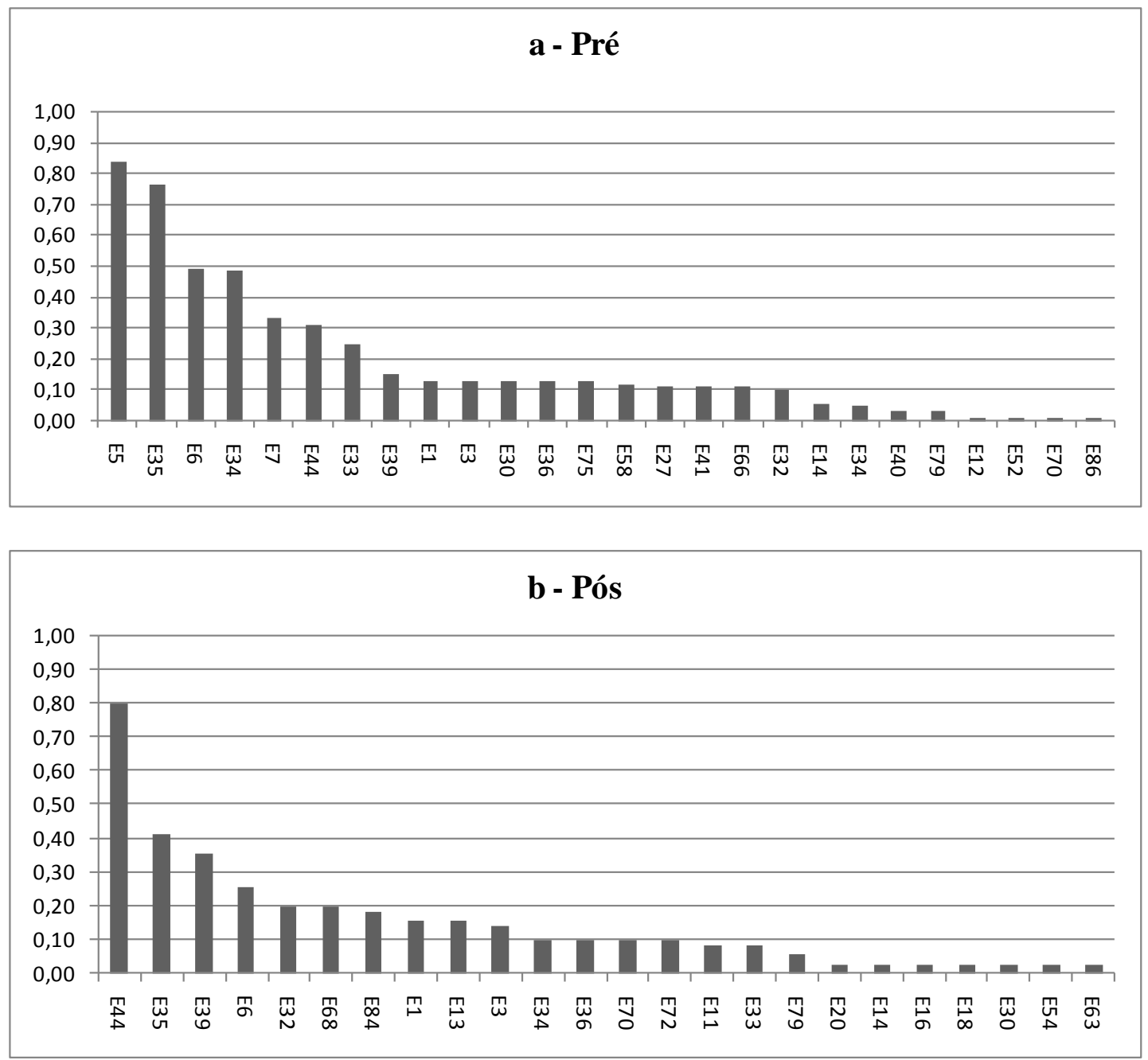

Figura 15. IAEP na estação de coleta Córrego Anhumas obtida nas coletas com aspirador elétrico, no monitoramento da Usina Hidrelétrica de Ourinhos, nos períodos pré (a) e pós (b) enchimento do reservatório.

Os valores do Índice de Abundância de Espécie Padronizado (IAEP) obtidos na técnica de coleta com armadilha de Shannon, disponibilizados nas Tabelas 10, 11 e 12 e ilustrados na Figura 16,17 e 18 a seguir apresentadas. 
Tabela 10. IAEP na estação de coleta Canteiro de Obras obtida nas coletas com armadilha de Shannon, no monitoramento da Usina Hidrelétrica de Ourinhos, nos períodos pré e pós enchimento do reservatório

\begin{tabular}{|c|c|c|}
\hline \multicolumn{3}{|l|}{ Pré } \\
\hline Espécie & Código & IAEP \\
\hline Cq. (Rhy.) chrysonotum/albifera & E25 & 0,73 \\
\hline Cq. (Rhy.) venezuelensis & E30 & 0,70 \\
\hline Ma. (Man.) titillans & E71 & 0,70 \\
\hline Cq. (Rhy.) juxtamansonia & E27 & 0,67 \\
\hline Ma. (Man.) wilsoni & E72 & 0,59 \\
\hline Cx. (Mel.) ribeirensis & E58 & 0,52 \\
\hline Ad. (Ady.) squamipennis & E1 & 0,47 \\
\hline Cq. (Rhy.) albicosta & E23 & 0,45 \\
\hline Cq. (Rhy.) hermanoi & E26 & 0,45 \\
\hline Cq. (Rhy.) chrysonotum & E24 & 0,44 \\
\hline Ae. (Och.) scapularis & E6 & 0,41 \\
\hline An. (Nys.) triannulatus & E18 & 0,40 \\
\hline An. (Nys.) galvaoi & E14 & 0,39 \\
\hline Cx. (Ads.) amazonenzis & E31 & 0,37 \\
\hline Cx. (Cux.) sp. & E44 & 0,33 \\
\hline Cq. (Rhy.) shannoni & E29 & 0,28 \\
\hline An. (Nys.) albitarsis s.l & E12 & 0,26 \\
\hline Cq. (Rhy.) nigricans & E28 & 0,23 \\
\hline Ps. (Jan.) ferox & E79 & 0,19 \\
\hline$C x .($ Cux.) grupo Coronator & E39 & 0,18 \\
\hline An. (Nys.) argyritarsis & E19 & 0,12 \\
\hline Cx. (Cux.) dolosus & E36 & 0,12 \\
\hline Cx. (Cux.) nigripalpus & E42 & 0,12 \\
\hline Ma. (Man.) amazonensis & E67 & 0,12 \\
\hline Cx. (Mel.) rabelloi & E57 & 0,12 \\
\hline Ps. (Jan.) albigenu & E73 & 0,12 \\
\hline Ae. (Och.) serratus & E7 & 0,07 \\
\hline An. (Nys.) evansae & E20 & 0,07 \\
\hline$C x$. (Mel.) seção Melanoconion & E59 & 0,05 \\
\hline Ur. (Ura.) pulcherrima & E84 & 0,05 \\
\hline Ae. (Och.) crinifer & E3 & 0,03 \\
\hline Ae.(Och.) fluviatilis & E4 & 0,03 \\
\hline Cx. (Cux.) quinquefascistus & E43 & 0,03 \\
\hline Cx. (Mel.) contei & E48 & 0,03 \\
\hline Cx. (Mel.) dureti & E50 & 0,03 \\
\hline Cx. (Mel.) plectoporpe & E56 & 0,03 \\
\hline Cx. (Mel.) dunni & E49 & 0,02 \\
\hline Ur. (Ura.) mathesoni & E87 & 0,02 \\
\hline Ma. (Man.) indubitans & E70 & 0,01 \\
\hline
\end{tabular}

\begin{tabular}{|c|c|c|}
\hline \multicolumn{3}{|l|}{$\begin{array}{r}\text { Pós } \\
\end{array}$} \\
\hline \multicolumn{3}{|l|}{ Espécie } \\
\hline Cq. (Rhy.) chrysonotum/albifera & E25 & 0,78 \\
\hline Ma. (Man.) wilsoni & E72 & 0,65 \\
\hline Cq. (Rhy.) juxtamansonia & E27 & 0,64 \\
\hline Cq. (Rhy.) hermanoi & E26 & 0,57 \\
\hline Cq. (Rhy.) venezuelensis & E30 & 0,51 \\
\hline Ma. (Man.) titillans & E71 & 0,49 \\
\hline Ae. (Och.) scapularis & E6 & 0,48 \\
\hline Cq. (Rhy.) chrysonotum & E24 & 0,45 \\
\hline Ad. (Ady.) squamipennis & E1 & 0,44 \\
\hline Cx. (Mel.) ribeirensis & E58 & 0,35 \\
\hline Cq. (Rhy.) nigricans & E28 & 0,34 \\
\hline Ps. (Jan.) ferox & E79 & 0,27 \\
\hline Ma. (Man.) amazonensis & E67 & 0,24 \\
\hline Cx. (Cux.) dolosus/eduardoi & E37 & 0,20 \\
\hline Cx. (Cux.) grupo Coronator & E39 & 0,18 \\
\hline Cq. (Rhy.) albicosta & E23 & 0,18 \\
\hline Cx. (Mel.) seção Melanoconion & E59 & 0,17 \\
\hline An. (Nys.) galvaoi & E14 & 0,16 \\
\hline Cx. (Cux.) nigripalpus & E42 & 0,15 \\
\hline Cx. (Cux.) sp. & E44 & 0,12 \\
\hline Cx. (Ads.) amazonenzis & E31 & 0,11 \\
\hline Cx. (Mel.) grupo A tratus & E52 & 0,11 \\
\hline An. strodei & E16 & 0,07 \\
\hline Cx. (Cux.) dolosus & E36 & 0,07 \\
\hline Cx. (Mel.) plectoporpe & E56 & 0,07 \\
\hline Ae. (Och.) serratus & E7 & 0,01 \\
\hline An. (Ano.) maculipes & E10 & 0,01 \\
\hline An. (Nys.) lutzii & E22 & 0,01 \\
\hline Cx. (Mel.) bastagarius & E47 & 0,01 \\
\hline Ma. (Man.) humeralis & E69 & 0,01 \\
\hline Ps. (Pso.) ciliata & E75 & 0,01 \\
\hline Ur. (Ura.) geometrica & E86 & 0,01 \\
\hline Ur. (Ura.) mathesoni & E87 & 0,01 \\
\hline Ur. (Ura.) nataliae & E88 & 0,01 \\
\hline Ur. pulcherrima & E84 & 0,01 \\
\hline Wy. sp. & E92 & 0,01 \\
\hline & & \\
\hline & & \\
\hline
\end{tabular}



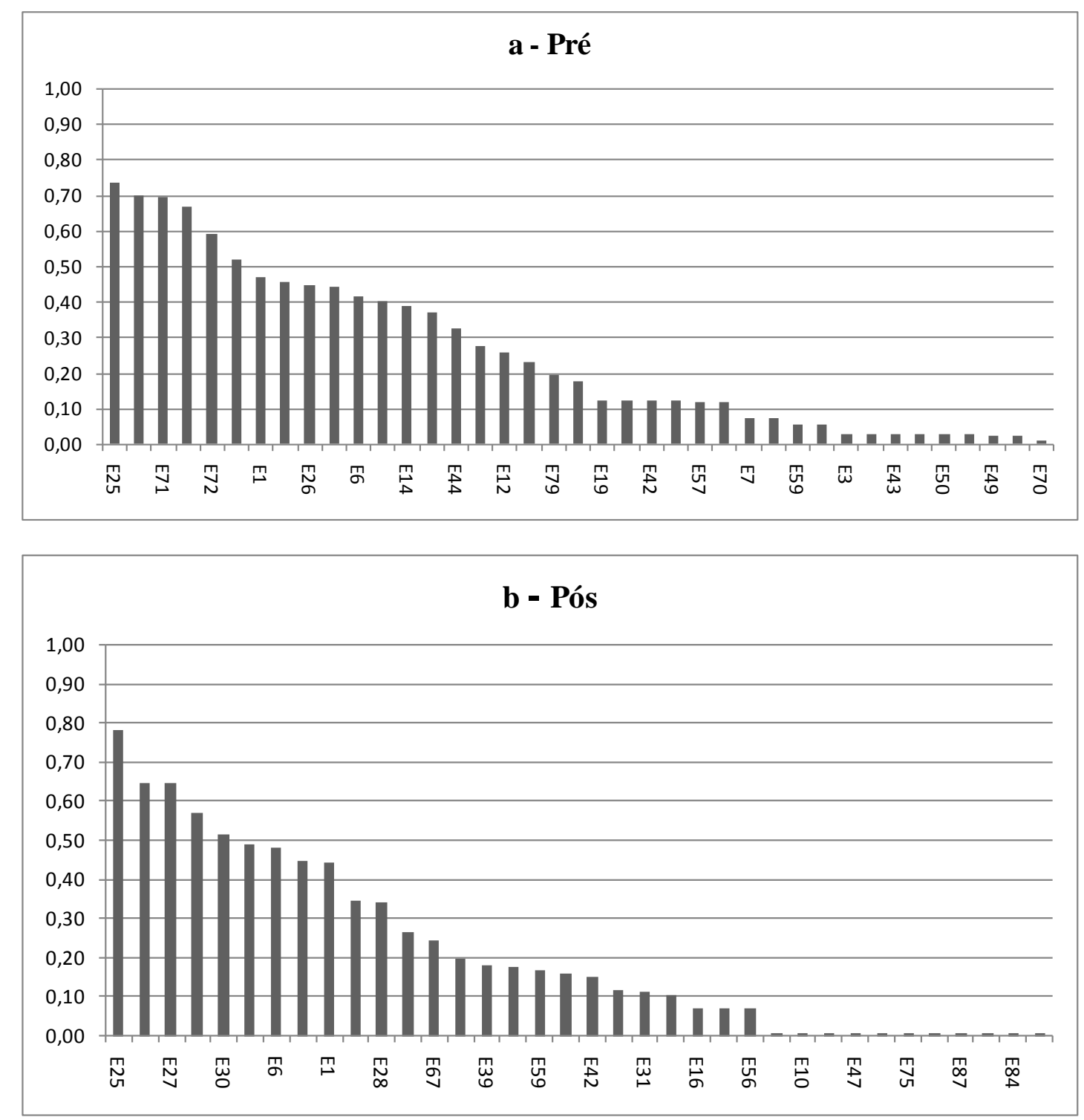

Figura 16. IAEP na estação de coleta Canteiro de Obras obtida nas coletas com armadilha de Shannon, no monitoramento da Usina Hidrelétrica de Ourinhos, nos períodos pré (a) e pós (b) enchimento do reservatório.

Observa-se na Tabela 10 uma leve redução no número de espécies, no período pós-inundação. Os gêneros Coquillettidia e Mansonia lideraram o “ranking” da abundância nas duas fases do estudo, sofrendo apenas alterações na ordem das espécies e um pequeno aumento nos valores de IAEP (Fig. 16). 
Tabela 11. IAEP na estação de coleta Sítio Caiçara obtida nas coletas com armadilha de Shannon, no monitoramento da Usina Hidrelétrica de Ourinhos, nos períodos pré e pós enchimento do reservatório.

\begin{tabular}{|c|c|c|}
\hline \multicolumn{3}{|l|}{ Pré } \\
\hline Espécie & Código & IAEP \\
\hline Cq. (Rhy.) juxtamansonia & E27 & 0,82 \\
\hline Ae. (Och.) scapularis & E6 & 0,72 \\
\hline Cq. (Rhy.) venezuelensis & E30 & 0,70 \\
\hline Cx. (Cux.) sp. & E44 & 0,54 \\
\hline$C x .(C u x$.$) grupo Coronator$ & E39 & 0,50 \\
\hline Cx. (Mel.) ribeirensis & E58 & 0,46 \\
\hline Ad. (Ady.) squamipennis & E1 & 0,38 \\
\hline Cq. (Rhy.) shannoni & E29 & 0,31 \\
\hline An. (Nys.) albitarsis s.l & E11 & 0,20 \\
\hline Ma. (Man.) titillans & E71 & 0,19 \\
\hline An. (Nys.) galvaoi & E14 & 0,18 \\
\hline Cq. (Rhy.) albicosta & E23 & 0,16 \\
\hline Cx. (Cux.) dolosus & E36 & 0,15 \\
\hline Ma. (Man.) wilsoni & E72 & 0,15 \\
\hline Cq. (Rhy.) hermanoi & E26 & 0,15 \\
\hline Ma. (Man.) indubitans & E70 & 0,13 \\
\hline Cx. (Mel.) grupo A tratus & E52 & 0,13 \\
\hline Ma. (Man.) humeralis & E69 & 0,12 \\
\hline Cx. (Cux.) nigripalpus & E42 & 0,11 \\
\hline Cq. (Rhy.) chrysonotum/albifera & E25 & 0,11 \\
\hline Ae. (Stg.) albopictus & E2 & 0,10 \\
\hline Cq. (Rhy.) nigricans & E28 & 0,08 \\
\hline Cx. (Cux.) declarator & E35 & 0,08 \\
\hline Ps. (Pso.) ciliata & E75 & 0,08 \\
\hline Ps. (Jan.) discrucians & E78 & 0,08 \\
\hline An. (Nys.) evansae & E20 & 0,05 \\
\hline Cx.(Mel.) flabellifer & E51 & 0,01 \\
\hline
\end{tabular}

\begin{tabular}{|l|l|l|}
\hline Espécie & Código & IAEP \\
\hline Cq. (Rhy.) juxtamansonia & E27 & 0,60 \\
\hline Cq. (Rhy.) venezuelensis & E30 & 0,54 \\
\hline Ae. (Och.) scapularis & $\mathrm{E} 6$ & 0,34 \\
\hline An. (Nys.) albitarsis s.l & $\mathrm{E} 11$ & 0,18 \\
\hline Cq. (Rhy.) albicosta & $\mathrm{E} 23$ & 0,16 \\
\hline Ma. (Man.) titillans & $\mathrm{E} 71$ & 0,16 \\
\hline Ma. (Man.) wilsoni & $\mathrm{E} 72$ & 0,16 \\
\hline Ad. (Ady.) squamipennis & $\mathrm{E} 1$ & 0,14 \\
\hline Cq. (Rhy.) shannoni & $\mathrm{E} 29$ & 0,14 \\
\hline Cx. (Cux.) sp. & $\mathrm{E} 44$ & 0,14 \\
\hline Ur. (Ura.) geometrica & $\mathrm{E} 86$ & 0,14 \\
\hline Ae. (Och.) crinifer & $\mathrm{E} 3$ & 0,11 \\
\hline Cq. (Rhy.) hermanoi & $\mathrm{E} 26$ & 0,11 \\
\hline Ae. (Stg.) albopictus & $\mathrm{E} 2$ & 0,02 \\
\hline Ae. (Och.) serratus & $\mathrm{E} 7$ & 0,02 \\
\hline An. (Nys.) galvaoi & $\mathrm{E} 14$ & 0,02 \\
\hline An. strodei & $\mathrm{E} 16$ & 0,02 \\
\hline Cq. (Rhy.) chrysonotum/albifera & $\mathrm{E} 25$ & 0,02 \\
\hline Cx. (Cux.) nigripalpus & $\mathrm{E} 42$ & 0,02 \\
\hline & & \\
\hline & & \\
\hline & & \\
\hline & & \\
\hline & & \\
\hline
\end{tabular}

Na Tabela 11, observa-se que houve drástica redução do número de espécies no período pós-enchimento do reservatório. Nota-se também na Figura17 marcante queda nos valores dos IAEPs após o fechamento das comportas da represa. Embora diante dessas alterações na fauna, as espécies Coquillettidia juxtamansonia, Coquillettidia venezuelensis e Aedes scapularis permaneceram nas primeiras posições nas duas situações. O mosquito Aedes albopictus esteve presente nas duas fases do estudo, porém como espécie rara. 

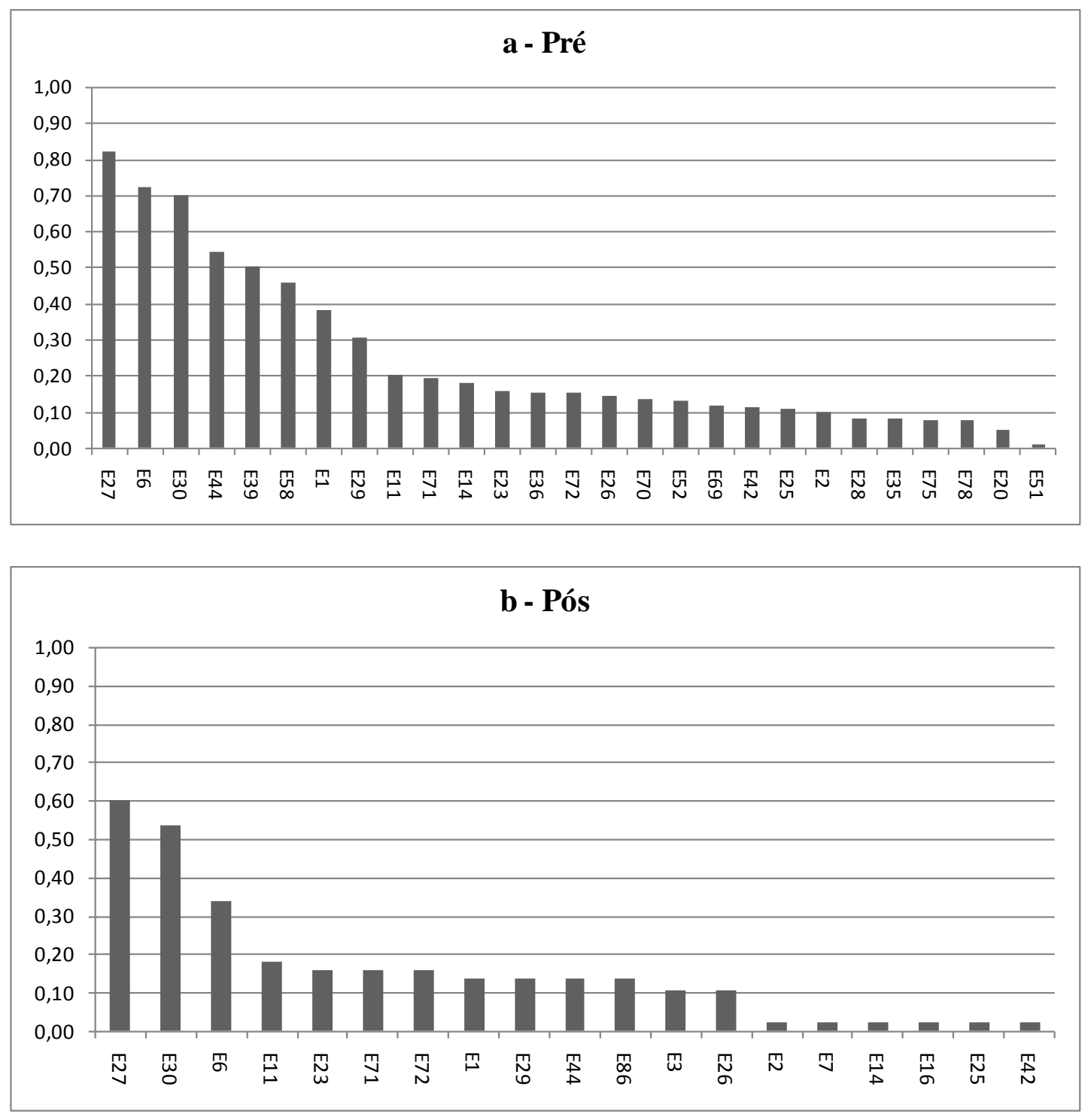

Figura 17. IAEP na estação de coleta Sítio Caiçara obtida nas coletas com armadilha de Shannon, no monitoramento da Usina Hidrelétrica de Ourinhos, nos períodos pré (a) e pós (b) enchimento do reservatório.

A Tabela 12 mostra um ligeiro aumento nos valores de IAEP da espécie mais abundante no período pós enchimento, e o aumento da riqueza nesse mesmo período. 
Tabela 12. IAEP na estação de coleta Córrego Anhumas obtida nas coletas com armadilha de Shannon, no monitoramento da Usina Hidrelétrica de Ourinhos, nos períodos pré e pós enchimento do reservatório

\begin{tabular}{|c|c|c|c|c|c|}
\hline \multicolumn{3}{|l|}{ Pré } & \multicolumn{3}{|l|}{ Pós } \\
\hline Espécie & Código & IAEP & Espécie & Código & IAEP \\
\hline Ae. (Och.) scapularis & E6 & 0,88 & Cq. (Rhy.) venezuelensis & E30 & 0,94 \\
\hline Cq. (Rhy.) venezuelensis & E30 & 0,67 & Ma. (Man.) wilsoni & E72 & 0,66 \\
\hline Ae. (Och.) serratus & E7 & 0,62 & An. (Nys.) albitarsis s.l & E11 & 0,65 \\
\hline Cq. (Rhy.) juxtamansonia & E27 & 0,61 & Ae. (Och.) scapularis & E6 & 0,51 \\
\hline Cx. (Cux.) sp. & E44 & 0,57 & Ad. (Ady.) squamipennis & E1 & 0,42 \\
\hline Cx. (Cux.) grupo Coronator & E39 & 0,48 & Cx. (Mel.) vaxus & E62 & 0,40 \\
\hline Ma. (Man.) wilsoni & E72 & 0,37 & An. strodei & E16 & 0,39 \\
\hline Ma. (Man.) titillans & E71 & 0,33 & Cx. (Mel.) aureonotatus & E46 & 0,36 \\
\hline Cq. (Rhy.) chrysonotum/albifera & E25 & 0,32 & Cq. (Rhy.) chrysonotum/albifera & E25 & 0,34 \\
\hline Ae. (Och.) crinifer & E3 & 0,28 & Cq. (Rhy.) juxtamansonia & E27 & 0,32 \\
\hline Ps. (Jan.) ferox & E79 & 0,26 & An. triannulatus & E18 & 0,31 \\
\hline CX. (Mel.) ribeirensis & E58 & 0,21 & $C x$. (Mel.) seção Melanoconion & E59 & 0,31 \\
\hline Cx. (Cux.) dolosus & E36 & 0,18 & Cq. (Rhy.) hermanoi & E26 & 0,30 \\
\hline An. (Nys.) evansae & E20 & 0,16 & Ma. (Man.) titillans & E71 & 0,28 \\
\hline Cq. (Rhy.) albicosta & E23 & 0,13 & Ae. (Och.) crinifer & E3 & 0,19 \\
\hline An. (Nys.) argyritarsis & E19 & 0,11 & An. (Nys.) evansae & E20 & 0,18 \\
\hline Cx. (Cux.) declarator & E35 & 0,11 & Cx. (Cux.) chidesteri & E33 & 0,18 \\
\hline Ae. (Och.) terrens & E9 & 0,11 & Cx. (Cux.) grupo Coronator & E39 & 0,17 \\
\hline Cx. (Cux.) chidesteri & E33 & 0,11 & An. (Nys.) galvaoi & E14 & 0,15 \\
\hline Cx. (Cux.) nigripalpus & E42 & 0,10 & Ps. (Jan.) discrucians & E78 & 0,15 \\
\hline Cx. (Cux.) coronator & E34 & 0,09 & \begin{tabular}{|l|} 
Ps. (Jan.) ferox \\
\end{tabular} & E79 & 0,15 \\
\hline Ps. (Jan.) albigenu & E73 & 0,08 & Ur. (Ura.) geometrica & E86 & 0,15 \\
\hline Ps. (Jan.) albipes & E74 & 0,08 & Cx. (Cux.) declarator & E35 & 0,14 \\
\hline An. strodei & E16 & 0,07 & Cx. (Cux.) sp. & E44 & 0,14 \\
\hline Cq. (Rhy.) shannoni & E29 & 0,07 & Cx. (Mel.) ocossa & E54 & 0,11 \\
\hline Cx. (Mel.) seção Melanoconion & E59 & 0,05 & Cx. (Mel.) bastagarius & E47 & 0,10 \\
\hline Ur. (Ura.) geometrica & E86 & 0,05 & Cx. (Mel.) plectoporpe & E56 & 0,10 \\
\hline An. (Nys.) albitarsis s.l & E11 & 0,04 & Cx. (Mel.) ribeirensis & E58 & 0,10 \\
\hline Cx. (Mel.) vaxus & E62 & 0,04 & Cx. (Mel.) dureti & E50 & 0,09 \\
\hline Hg. (Con.) leucocelaenus & E65 & 0,04 & Cq. (Rhy.) nigricans & E28 & 0,08 \\
\hline Ma. (Man.) indubitans & E70 & 0,02 & Cq. (Rhy.) shannoni & E29 & 0,05 \\
\hline Ps. (Pso.) ciliata & E75 & 0,02 & An. darlingi & E13 & 0,01 \\
\hline Ad. (Ady.) squamipennis & E1 & 0,01 & An. (Ano.) fluminensis & E21 & 0,01 \\
\hline An. (Nys.) galvaoi & E14 & 0,01 & Cx. (Cux.) dolosus & E36 & 0,01 \\
\hline Cq. (Rhy.) hermanoi & E26 & 0,01 & Cx. (Mel.) idottus & E53 & 0,01 \\
\hline Ps. (Jan.) discrucians & E78 & 0,01 & Cx. (Mel.) pilosus & E55 & 0,01 \\
\hline & & & Ma. (Man.) indubitans & E70 & 0,01 \\
\hline
\end{tabular}



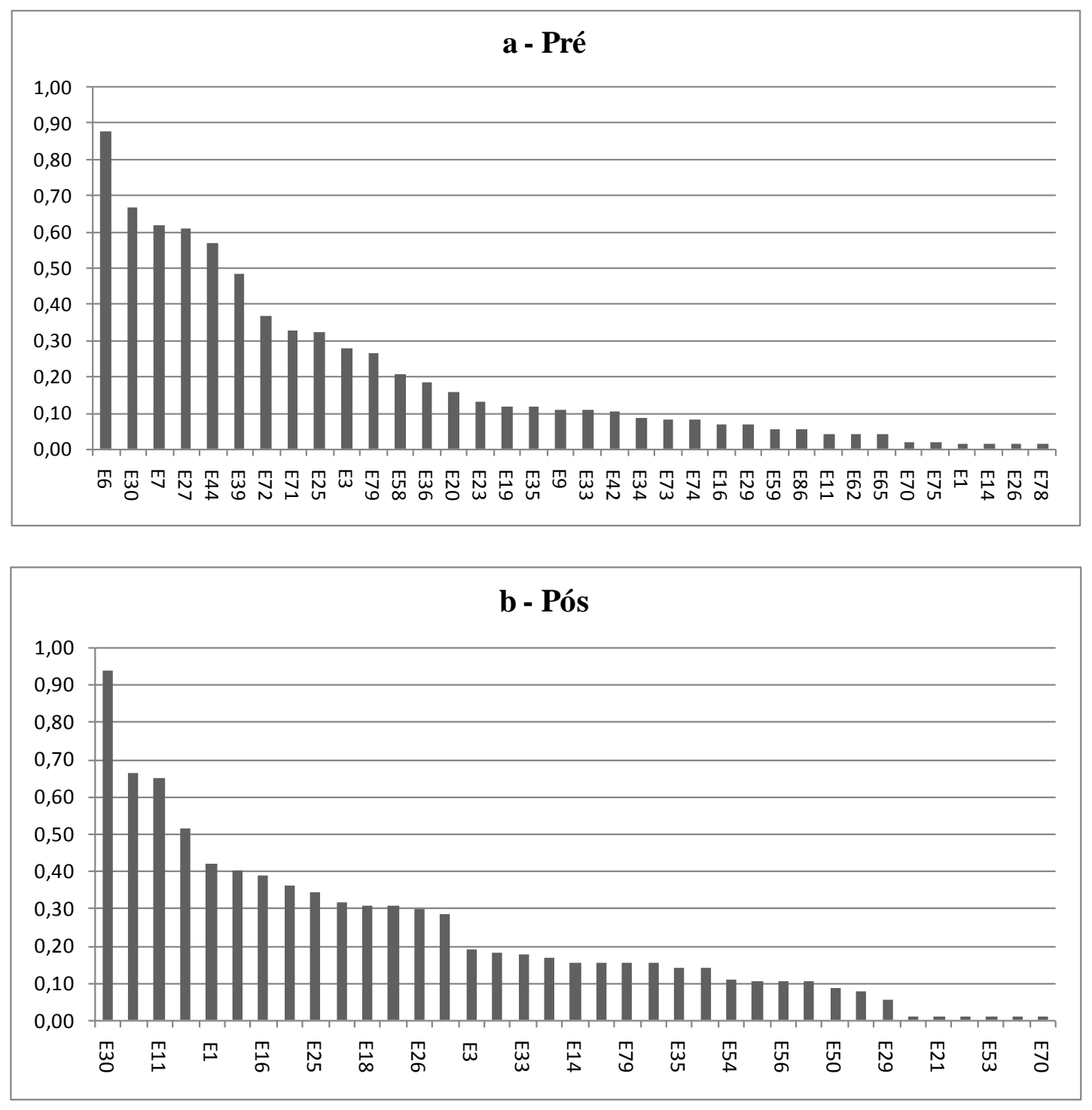

Figura 18. IAEP na estação de coleta Córrego Anhumas obtida nas coletas com armadilha de Shannon, no monitoramento da Usina Hidrelétrica de Ourinhos, nos períodos pré (a) e pós (b) enchimento do reservatório.

Na observação da Figura 18 fica evidente a semelhança, nas duas fases, do número de espécies. As espécies Aedes scapularis e Coquillettidia venezuelensis estiveram entre as mais abundantes nos dois períodos de estudo. Entre os anofelinos, destaca-se a alteração na abundância de Anopheles albitarsis s.l, que antes do período de inundação ocupou a posição 28, enquanto na fase posterior inundada foi para a posição 3. Destaca-se também, o surgimento de Anopheles darlingi, não 
capturado na primeira fase e ocupando a posição de abundância número 32 no período pós enchimento.

Os valores do Índice de Abundância de Espécie Padronizado obtidos pela somatória dos resultados das três técnicas para cada estação de coleta estão, disponibilizados nas Tabelas 13, 14 e 15 e ilustrados na Figura 19,20 e 21.

As Tabelas 13, 14 e 15, mostram que não houve alterações drásticas no número de espécies das estações de coleta com o fechamento da barragem e posterior enchimento do reservatório.

Na Figura 19 observa-se que a tendência de distribuição dos valores de IAEP das espécies nos períodos pré e pós não se alteraram, sendo em ambos os períodos encontradas oito espécies com IAEP maior que 0,50. Culex (Cux.) grupo Coronator subiu da $12^{\mathrm{a}}$ para a $1^{\mathrm{a}}$ posição no ranking, Coquillettidia (Rhy.) chrysonotum/albifera manteve o IAEP de 0,75 indo da $1^{\mathrm{a}}$ para a $2^{\mathrm{a}}$ posição e Anopheles (Nys.) galvaoi foi da $8^{\mathrm{a}}$ para a $22^{\mathrm{a}}$.

Sobressai na Figura 20 a redução nos valores de IAEP sendo os valores máximos 0,96 e 0,66, nos períodos pré e pós, respectivamente. Anopheles albitarsis s.l pulou da $12^{\mathrm{a}}$ posição para a $1^{\mathrm{a}}$, enquanto Culex (Cux.) grupo Coronator teve diminuição de 0,96 para 0,43 nos valor de IAEP e Anopheles (Nys.) darlingi aparece somente no período pós na $24^{\mathrm{a}}$ posição.

A análise conjunta da Tabela 15 e da Figura 21 mostra uma redução no número de espécies e um aumento nos valores do IAEP. Limatus durhami e Haemagogus leucocelaenus, encontrados no período pré, não aparecem no período pós. Em contrapartida Anopheles (Nys.) darlingi e Culex quinquefasciatus estão presentes apenas no pós enchimento, ocupado a $31^{\mathrm{a}}$ e $39^{\mathrm{a}}$ posições, respectivamente. 
Tabela 13. IAEP na estação de coleta Canteiro de Obras, obtida pelas três técnicas de coleta, no monitoramento da Usina Hidrelétrica de Ourinhos, nos períodos pré e pós enchimento do reservatório.

\begin{tabular}{|c|c|c|c|c|c|}
\hline \multicolumn{3}{|l|}{ Pré } & \multicolumn{3}{|l|}{ Pós } \\
\hline Espécie & Código & IAEP & Espécie & Código & IAEP \\
\hline Cq. (Rhy.) chrysonotum/albifera & E25 & 0,75 & Cx. (Cux.) grupo Coronator & E39 & 0,79 \\
\hline Cq. (Rhy.) venezuelensis & E30 & 0,71 & Cq. (Rhy.) chrysonotum/albifera & E25 & 0,75 \\
\hline Ma. (Man.) titillans & E71 & 0,68 & CX. (Cux.) coronator & E34 & 0,68 \\
\hline Cq. (Rhy.) juxtamansonia & E27 & 0,67 & Cx. (Cux.) sp. & E44 & 0,58 \\
\hline Cx. (Cux.) sp. & E44 & 0,63 & Ma. (Man.) wilsoni & E72 & 0,58 \\
\hline Ma. (Man.) wilsoni & E72 & 0,60 & Ae. (Och.) scapularis & E6 & 0,57 \\
\hline An. (Nys.) galvaoi & E14 & 0,58 & Cq. (Rhy.) hermanoi & E26 & 0,56 \\
\hline CX. (Mel.) ribeirensis & E58 & 0,57 & Cq. (Rhy.) juxtamansonia & E27 & 0,53 \\
\hline Cq. (Rhy.) albicosta & E23 & 0,47 & Cx. (Cux.) dolosus & E36 & 0,49 \\
\hline Ad. (Ady.) squamipennis & E1 & 0,47 & Ur. (Ura.) geometrica & E86 & 0,47 \\
\hline Cq. (Rhy.) hermanoi & E26 & 0,45 & Cq. (Rhy.) venezuelensis & E30 & 0,44 \\
\hline Cq. (Rhy.) chrysonotum & E24 & 0,45 & Ma. (Man.) titillans & E71 & 0,42 \\
\hline Cx. (Cux.) grupo Coronator & E39 & 0,45 & Cq. (Rhy.) chrysonotum & E24 & 0,41 \\
\hline Ae. (Och.) scapularis & E6 & 0,42 & Ad. (Ady.) squamipennis & E1 & 0,39 \\
\hline An. (Nys.) triannulatus & E18 & 0,38 & An. (Nys.) argyritarsis & E19 & 0,32 \\
\hline An. (Nys.) argyritarsis & E19 & 0,34 & $C x .($ Mel.) ribeirensis & E58 & 0,32 \\
\hline CX. (Ads.) amazonenzis & E31 & 0,31 & Cq. (Rhy.) nigricans & E28 & 0,32 \\
\hline Cx. (Cux.) coronator & E34 & 0,31 & An. (Nys.) albitarsis s.l & E11 & 0,31 \\
\hline Ae. (Och.) fluviatilis & E4 & 0,30 & Ur. (Ura.) mathesoni & E87 & 0,27 \\
\hline Cq. (Rhy.) shannoni & E29 & 0,28 & Cx. (Cux.) declarator & E35 & 0,25 \\
\hline An. (Nys.) albitarsis s.l & E11 & 0,26 & Ur. (Ura.) lowii & E83 & 0,25 \\
\hline Ur. (Ura.) mathesoni & E87 & 0,25 & \begin{tabular}{|l|} 
Ps. (Jan.) ferox \\
\end{tabular} & E79 & 0,24 \\
\hline Ps. (Jan.) ferox & E79 & 0,24 & Ur. (Ura.) pulcherrima & E84 & 0,24 \\
\hline Ur. (Ura.) geometrica & E86 & 0,22 & Ma. (Man.) amazonensis & E67 & 0,21 \\
\hline Cq. (Rhy.) nigricans & E28 & 0,21 & Cx. (Mel.) grupo A tratus & E52 & 0,21 \\
\hline Ps. (Jan.) albigenu & E73 & 0,18 & Ur. (Ura.) nataliae & E88 & 0,20 \\
\hline An. (Nys.) strodei/ rondoni & E17 & 0,18 & Wy. (Den.) rooti & E91 & 0,19 \\
\hline Cx. (Cux.) quinquefascistus & E43 & 0,18 & Wy. sp. & E92 & 0,19 \\
\hline Ur. (Ura.) nataliae & E88 & 0,17 & Cx. (Cux.) dolosus/eduardoi & E37 & 0,18 \\
\hline Cx. (Cux.) dolosus & E36 & 0,17 & Ae. fluviatilis & $\mathrm{E} 4$ & 0,18 \\
\hline Ur. (Ura.) pulcherrima & E84 & 0,15 & Cx. (Mcx.) imitator & E45 & 0,18 \\
\hline Cx. (Cux.) declarator & E35 & 0,14 & Cq. (Rhy.) albicosta & E23 & 0,18 \\
\hline Wy. sp. & E92 & 0,14 & Cx. (Mel.) seção Melanoconion & E59 & 0,17 \\
\hline Cx. (Mel.) seção Melanoconion & E59 & 0,14 & Cx. (Cux.) nigripalpus & E42 & 0,15 \\
\hline Cx.(Cux.) nigripalpus & E42 & 0,13 & Cx. (Cux.) quinquefascistus & E43 & 0,14 \\
\hline Ps. (Pso.) ciliata & E75 & 0,12 & An. (Nys.) galvaoi & E14 & 0,14 \\
\hline Cx. (Cux.) chidesteri & E33 & 0,11 & Cx. (Cux.) bidens & E32 & 0,11 \\
\hline Ma. (Man.) amazonensis & E67 & 0,11 & Sa.sp. & E82 & 0,11 \\
\hline Ps. (Gra.) confinnis & E77 & 0,10 & An. (Nys.) sp & E15 & 0,11 \\
\hline An. (Nys.) sp & E15 & 0,10 & Cx. (Ads.) amazonenzis & E31 & 0,11 \\
\hline Cx. (Mel.) rabelloi & E57 & 0,10 & Ae. (Stg.) albopictus & E2 & 0,09 \\
\hline Cx. (Cux.) dolosus/eduardoi & E37 & 0,08 & An. strodei & E16 & 0,06 \\
\hline Ae. (Och.) serratus & E7 & 0,08 & Cx. (Mel.) plectoporpe & E56 & 0,06 \\
\hline An. (Nys.) evansae & E20 & 0,08 & Ae. (Och.) serratus & E7 & 0,01 \\
\hline Cx. (Cux.) mollis & E41 & 0,07 & An. (Ano.) maculipes & E10 & 0,01 \\
\hline Ps. (Jan.) lutzii & E80 & 0,05 & An. (Nys.) lutzii & E22 & 0,01 \\
\hline Ae. (Stg.) albopictus & E2 & 0,04 & Cx. (Mel.) bastagarius & E47 & 0,01 \\
\hline Cx. (Mel.) plectoporpe & E56 & 0,04 & Cx. (Mel.) rabelloi & E57 & 0,01 \\
\hline Ae. (Och.) crinifer & E3 & 0,03 & Cx. (Mel.) sphatulatus & E61 & 0,01 \\
\hline Cx. (Mel.) contei & E48 & 0,03 & Ma. (Man.) humeralis & E69 & 0,01 \\
\hline Cx. (Mel.) dureti & E50 & 0,03 & Ps. (Pso.) ciliata & E75 & 0,01 \\
\hline Cx. (Mel.) ocossa & E54 & 0,03 & & & \\
\hline Ma. (Man.) indubitans & E70 & 0,03 & & & \\
\hline CX. (Mel.) dunni & E49 & 0,01 & & & \\
\hline
\end{tabular}



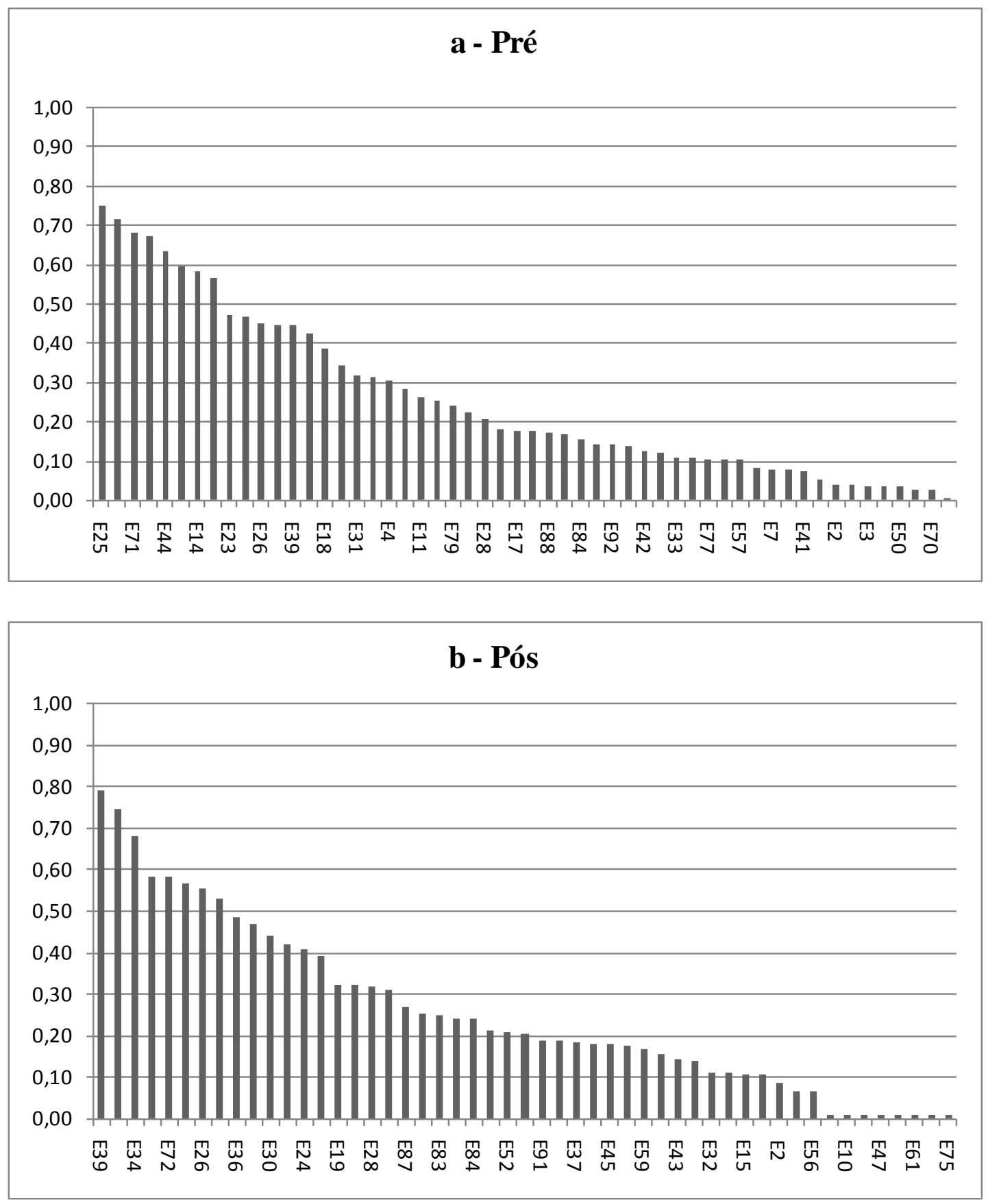

Figura 19. IAEP na estação de coleta Canteiro de Obras obtida pelas três técnicas de coleta, no monitoramento da Usina Hidrelétrica de Ourinhos, nos períodos pré (a) e pós (b) enchimento do reservatório. 
Tabela 14. IAEP na estação de coleta Sítio Caiçara obtida pelas três técnicas de coleta, no monitoramento da Usina Hidrelétrica de Ourinhos, nos períodos pré e pós enchimento do reservatório.

\begin{tabular}{|c|c|c|c|c|c|}
\hline \multicolumn{3}{|l|}{ Pré } & \multicolumn{3}{|l|}{ Pós } \\
\hline Espécie & Código & IAEP & Espécie & Código & IAEP \\
\hline Cx. (Cux.) grupo Coronator & E39 & 0,96 & An. (Nys.) albitarsis s.l & E11 & 0,66 \\
\hline Ae. (Och.) scapularis & E6 & 0,81 & Cq. (Rhy.) venezuelensis & E30 & 0,57 \\
\hline Cq. (Rhy.) juxtamansonia & E27 & 0,76 & Ad. (Ady.) squamipennis & E1 & 0,55 \\
\hline Cx. (Cux.) sp. & E44 & 0,67 & Cq. (Rhy.) juxtamansonia & E27 & 0,54 \\
\hline Cq. (Rhy.) venezuelensis & E30 & 0,61 & Cx. (Cux.) sp. & E44 & 0,44 \\
\hline Cx. (Cux.) coronator & E34 & 0,57 & $C x .($ Cux.) grupo Coronator & E39 & 0,43 \\
\hline Cx. (Cux.) declarator & E35 & 0,57 & An. (Nys.) argyritarsis & E19 & 0,43 \\
\hline Cx. (Cux.) dolosus & E36 & 0,51 & Cx. (Cux.) coronator & E34 & 0,37 \\
\hline Ae. (Stg.) albopictus & E2 & 0,49 & Cx. (Cux.) chidesteri & E33 & 0,33 \\
\hline CX. (Mel.) ribeirensis & E58 & 0,37 & Ae. (Och.) scapularis & E6 & 0,28 \\
\hline Ad. (Ady.) squamipennis & E1 & 0,28 & An. (Nys.) strodei & E16 & 0,27 \\
\hline An. (Nys.) albitarsis s.l & E11 & 0,27 & Cx. (Cux.) mollis & E41 & 0,27 \\
\hline Cq. (Rhy.) shannoni & E29 & 0,25 & An. (Nys.) sp & E15 & 0,24 \\
\hline Cx. (Cux.) dolosus/eduardoi & E37 & 0,23 & Ae. (Och.) fluviatilis & E4 & 0,20 \\
\hline Ae. (Och.) terrens & E9 & 0,21 & Cx. (Mel.) sp & E60 & 0,19 \\
\hline An. (Nys.) galvaoi & E14 & 0,19 & An. (Nys.) triannulatus & E18 & 0,16 \\
\hline Cx. (Cux.) mollis & E41 & 0,19 & Ae. (Och.) crinifer & E3 & 0,15 \\
\hline An. (Nys.) strodei/ rondoni & E17 & 0,19 & Cx. (Cux.) dolosus & E36 & 0,13 \\
\hline Ma. (Man.) titillans & E71 & 0,15 & Cx. (Mel.) aureonotatus & E46 & 0,13 \\
\hline Ma. (Man.) wilsoni & E72 & 0,13 & Ur. (Ura.) lowii & E82 & 0,13 \\
\hline Cq. (Rhy.) hermanoi & E26 & 0,13 & Cx. (Mel.) vaxus & E62 & 0,11 \\
\hline Cq. (Rhy.) albicosta & E23 & 0,13 & Ae. (Och.) serratus/nubilus & E8 & 0,11 \\
\hline Cx. (Cux.) lygrus & E40 & 0,12 & Wy. sp. & E92 & 0,11 \\
\hline Cq. (Rhy.) chrysonotum/albifera & E25 & 0,12 & An. (Nys.) darlingi & E13 & 0,09 \\
\hline Cx. (Cux.) nigripalpus & E42 & 0,11 & Cx. (Cux.) dolosus/eduardoi & E37 & 0,09 \\
\hline Cx. (Cux.) eduardoi & E38 & 0,11 & Ae. (Och.) serratus & E7 & 0,07 \\
\hline Ma. (Man.) indubitans & E70 & 0,11 & Cq. (Rhy.) albicosta & E23 & 0,07 \\
\hline Cx. (Cux.) quinquefascistus & E43 & 0,10 & Cq. (Rhy.) hermanoi & E26 & 0,07 \\
\hline Cx. (Cux.) chidesteri & E33 & 0,09 & Ma. (Man.) titillans & E71 & 0,07 \\
\hline Wy. sp. & E92 & 0,09 & Ma. (Man.) wilsoni & E72 & 0,07 \\
\hline Ps. (Jan.) discrucians & E78 & 0,08 & Cx. (Mel.) seção Melanoconion & E59 & 0,07 \\
\hline Ps. (Pso.) ciliata & E75 & 0,08 & Cq. (Rhy.) shannoni & E29 & 0,02 \\
\hline CX. (Mel.) grupo A tratus & E52 & 0,08 & Cx. (Mel.) dureti & E50 & 0,02 \\
\hline Cx. (Mel.) ocossa & E54 & 0,07 & Ur. (Ura.) geometrica & E86 & 0,02 \\
\hline Ma. (Man.) humeralis & E69 & 0,07 & Ur. (Ura.) sp. & E85 & 0,02 \\
\hline An. (Nys.) evansae & E20 & 0,05 & Ae. (Och.) nubilus & E5 & 0,01 \\
\hline An. (Nys.) argyritarsis & E19 & 0,04 & An. (Nys.) galvaoi & E14 & 0,01 \\
\hline Cq. (Rhy.) nigricans & E28 & 0,04 & Cq. (Rhy.) chrysonotum/albifera & E25 & 0,01 \\
\hline Ur. (Ura.) pulcherrima & E84 & 0,04 & Cx. (Cux.) lygrus & E40 & 0,01 \\
\hline Cx. (Mel.) pilosus & E55 & 0,03 & Cx. (Cux.) nigripalpus & E42 & 0,01 \\
\hline Cx. (Mel.) flabellifer & E51 & 0,01 & Ae. (Stg.) albopictus & E2 & 0,01 \\
\hline
\end{tabular}



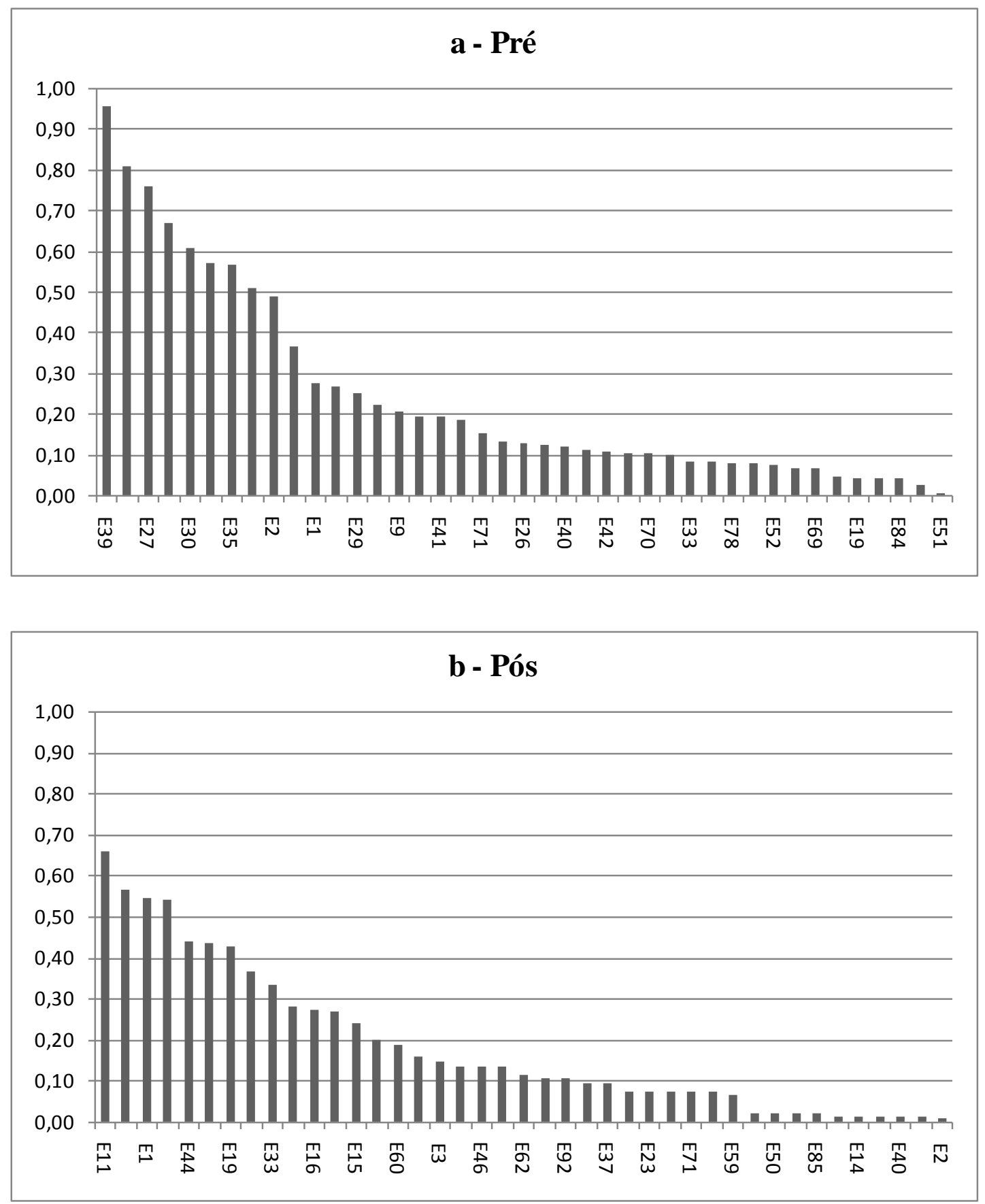

Figura 20. IAEP na estação de coleta Sítio Caiçara obtida pelas três técnicas de coleta, no monitoramento da Usina Hidrelétrica de Ourinhos, nos períodos pré (a) e pós (b) enchimento do reservatório. 
Tabela 15. IAEP na estação de coleta Córrego Anhumas obtida pelas três técnicas de coleta, no monitoramento da Usina Hidrelétrica de Ourinhos, nos períodos pré e pós enchimento do reservatório.

\begin{tabular}{|c|c|c|c|c|c|}
\hline \multicolumn{3}{|l|}{ Pré } & \multicolumn{3}{|l|}{ Pós } \\
\hline Espécie & Código & IAEP & Espécie & Código & IAEP \\
\hline Ae. (Och.) scapularis & E6 & 0,91 & Ad. (Ady.) squamipennis & E1 & 0,95 \\
\hline$C x .($ Cux.) grupo Coronator & E39 & 0,74 & An. (Nys.) albitarsis s.l & E11 & 0,90 \\
\hline Cx. (Cux.) sp. & E44 & 0,68 & Cq. (Rhy.) venezuelensis & E30 & 0,86 \\
\hline Cq. (Rhy.) venezuelensis & E30 & 0,65 & Cx. (Cux.) sp. & E44 & 0,80 \\
\hline Ae. (Och.) serratus & E7 & 0,63 & Ae. (Och.) scapularis & E6 & 0,64 \\
\hline Cq. (Rhy.) juxtamansonia & E27 & 0,59 & Ma. (Man.) wilsoni & E72 & 0,62 \\
\hline Cx. (Cux.) coronator & E34 & 0,58 & Cx. (Mel.) seção Melanoconion & E59 & 0,61 \\
\hline Cx. (Cux.) declarator & E35 & 0,56 & $C x .($ Cux.) grupo Coronator & E39 & 0,50 \\
\hline An. (Nys.) argyritarsis & E19 & 0,52 & An. (Nys.) strodei & E16 & 0,43 \\
\hline Cx.(Cux.) chidesteri & E33 & 0,51 & Cx. (Mel.) vaxus & E62 & 0,40 \\
\hline Cx. (Cux.) dolosus & E36 & 0,49 & Cx. (Mel.) aureonotatus & E46 & 0,38 \\
\hline Ad. (Ady.) squamipennis & E1 & 0,41 & Cq. (Rhy.) chrysonotum/albifera & E25 & 0,34 \\
\hline Ma. (Man.) wilsoni & E72 & 0,40 & An. (Nys.) triannulatus & E18 & 0,31 \\
\hline Ma. (Man.) titillans & E71 & 0,36 & Cq. (Rhy.) juxtamansonia & E27 & 0,30 \\
\hline Ae. (Och.) crinifer & E3 & 0,35 & Ma. (Man.) titillans & E71 & 0,30 \\
\hline An. (Nys.) albitarsis s.l & E11 & 0,34 & Cq. (Rhy.) hermanoi & E26 & 0,29 \\
\hline Cq. (Rhy.) chrysonotum/albifera & E25 & 0,33 & Cx. (Cux.) declarator & E35 & 0,28 \\
\hline Ps. (Jan.) ferox & E79 & 0,33 & Cx. (Cux.) chidesteri & E33 & 0,25 \\
\hline CX. (Mel.) ribeirensis & E58 & 0,32 & Cx. (Cux.) coronator & E34 & 0,23 \\
\hline Ur. (Ura.) geometrica & E86 & 0,23 & An. (Nys.) evansae & E20 & 0,22 \\
\hline Ur. (Ura.) pulcherrima & E84 & 0,18 & Ae. (Och.) crinifer & E3 & 0,19 \\
\hline Wy. (Wye.) medioalbipes & E90 & 0,16 & Cx. (Mel.) ocossa & E54 & 0,19 \\
\hline Cq. (Rhy.) albicosta & E23 & 0,16 & Ur. (Ura.) pulcherrima & E84 & 0,18 \\
\hline An. (Nys.) evansae & E20 & 0,15 & Cx. (Cux.) bidens & E32 & 0,16 \\
\hline An. (Nys.) galvaoi & E14 & 0,15 & An. (Nys.) galvaoi & E14 & 0,16 \\
\hline Cx. (Mel.) vaxus & E62 & 0,14 & Ps. (Jan.) ferox & E79 & 0,15 \\
\hline Ps. (Pso.) ciliata & E75 & 0,14 & An. (Nys.) argyritarsis & E19 & 0,14 \\
\hline Ae. (Och.) terrens & E9 & 0,14 & Ps. (Jan.) discrucians & E78 & 0,13 \\
\hline Ur. (Ura.) lowii & E83 & 0,14 & Ur. (Ura.) geometrica & E86 & 0,13 \\
\hline Cx. (Mcx.) imitator & E45 & 0,13 & An. (Nys.) darlingi & E13 & 0,11 \\
\hline CX. (Mel.) seção Melanoconion & E59 & 0,12 & Cq. (Rhy.) nigricans & E28 & 0,10 \\
\hline Ae. (Och.) nubilus & E5 & 0,12 & CX. (Mel.) bastagarius & E47 & 0,10 \\
\hline CX. (Cux.) nigripalpus & E42 & 0,11 & CX. (Mel.) plectoporpe & E56 & 0,10 \\
\hline An. (Nys.) sp & E15 & 0,09 & Cx. (Mel.) ribeirensis & E58 & 0,10 \\
\hline Ps. (Jan.) albigenu & E73 & 0,08 & CX. (Mel.) dureti & E50 & 0,09 \\
\hline Ps. (Jan.) albipes & E74 & 0,08 & Ma. (Man.) flaveola & E68 & 0,09 \\
\hline Ps. (Gra.) cingulata & E76 & 0,08 & Cq. (Rhy.) shannoni & E29 & 0,07 \\
\hline Ps. sp. & E81 & 0,08 & Cx. (Cux.) quinquefascistus & E43 & 0,07 \\
\hline Cx. (Mel.) bastagarius & E47 & 0,07 & Cx. (Mel.) zeteki & E63 & 0,07 \\
\hline Cx. (Cux.) saltanensis & E64 & 0,07 & An. (Nys.) $s p$ & E15 & 0,06 \\
\hline Hg. (Con.) leucocelaenus & E65 & 0,07 & Cx.(Cux.) dolosus & E36 & 0,06 \\
\hline An. (Nys.) strodei & E16 & 0,06 & An. (Ano.) fluminensis & E21 & 0,01 \\
\hline Cx.(Cux.) lygrus & E40 & 0,06 & Cx. (Mel.) idottus & E53 & 0,01 \\
\hline Ps. (Gra.) confinnis & E77 & 0,06 & Cx. (Mel.) pilosus & E55 & 0,01 \\
\hline Cq. (Rhy.) shannoni & E29 & 0,05 & Ma. (Man.) indubitans & E70 & 0,01 \\
\hline Cx. (Mel.) sp. & E60 & 0,05 & Ur. sp & E89 & 0,01 \\
\hline Cx. (Cux.) bidens & E32 & 0,05 & & & \\
\hline Ma. (Man.) indubitans & E70 & 0,04 & & & \\
\hline CX. (Cux.) mollis & E41 & 0,04 & & & \\
\hline Li. durhami & E66 & 0,04 & & & \\
\hline Cq. (Rhy.) hermanoi & E26 & 0,01 & & & \\
\hline$C x .($ Mel.) grupo A tratus & E52 & 0,01 & & & \\
\hline Ps. (Jan.) discrucians & E78 & 0,01 & & & \\
\hline
\end{tabular}



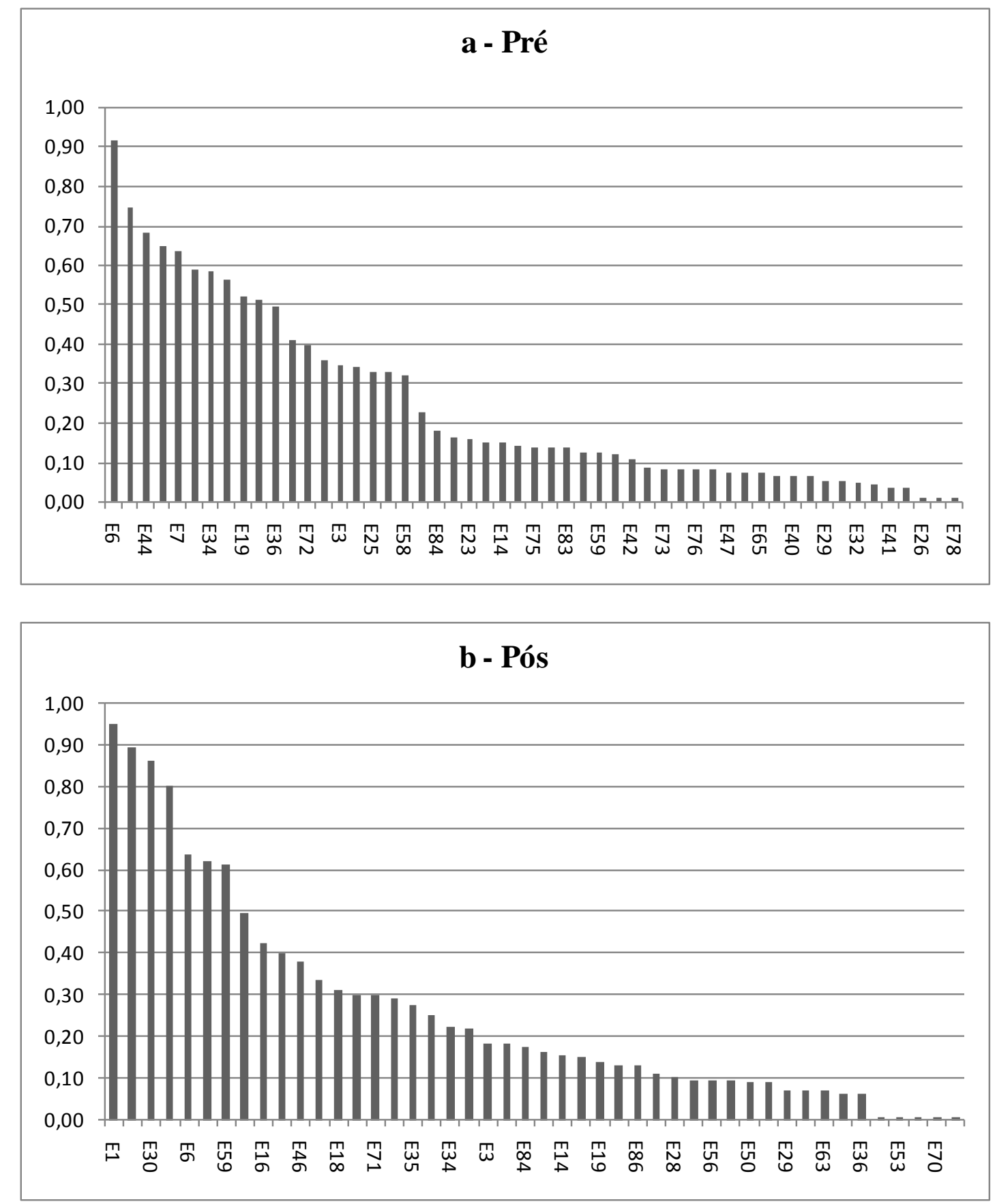

Figura 21. IAEP na estação de coleta Córrego Anhumas obtida pelas três técnicas de coleta, no monitoramento da Usina Hidrelétrica de Ourinhos, nos períodos pré (a) e pós (b) enchimento do reservatório. 


\subsection{Similaridade}

O índice de Similaridade de Jaccard varia entre 0 e 1, sendo 0 a total ausência de espécies em comum e 1 que as amostras apresentam a mesma composição quanto às espécies.

\subsubsection{Espacial}

No período pré enchimento Canteiro de Obras/Sítio Caiçara (CO/SC) apresentava maior índice de similaridade $(\mathrm{Ij}=0,42)$. As estações que sofreram influência direta da formação do reservatório, Sítio Caiçara/Córrego Anhumas (SC/CA), tiveram aumento da similaridade apresentando assim um $\mathrm{Ij}=0,52$.

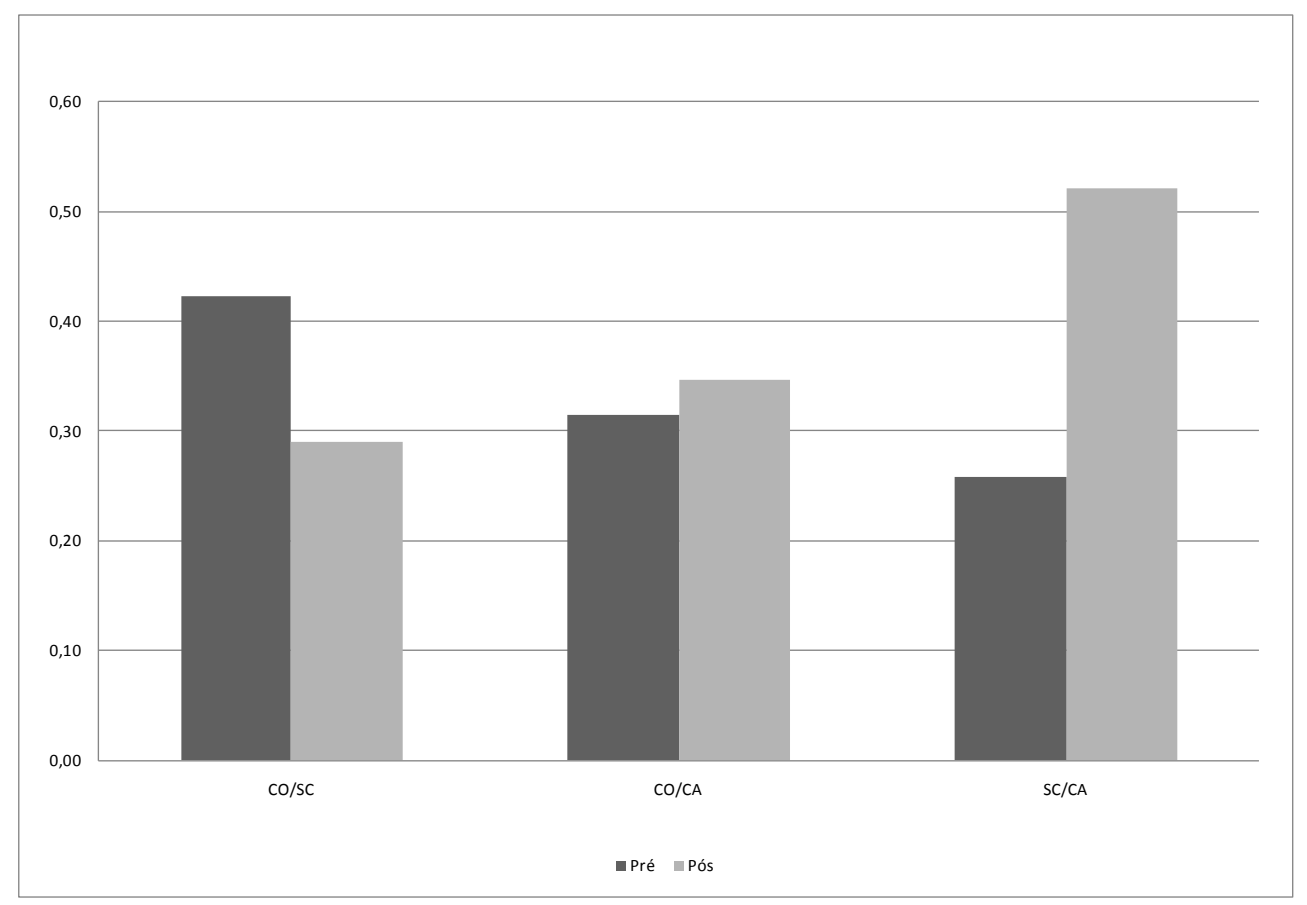

Figura 22. Coeficiente de Similaridade de Jaccard entre estações de coleta, obtida nas coletas com concha entomológica para avaliação de formas imaturas, no monitoramento da Usina Hidrelétrica de Ourinhos, nos períodos pré e pós enchimento do reservatório. 


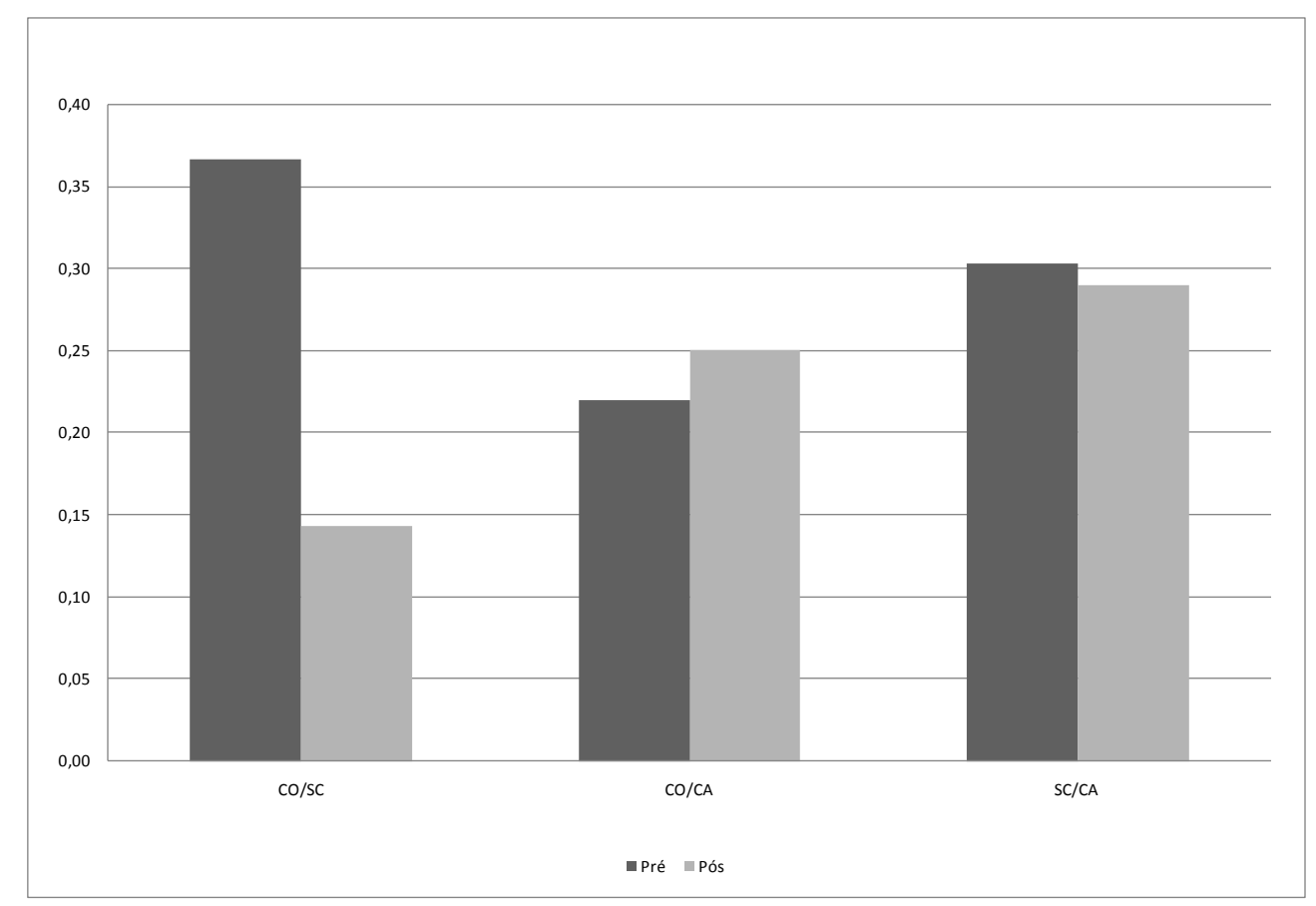

Figura 23. Coeficiente de Similaridade de Jaccard entre estações de coleta, obtida nas coletas com aspirador elétrico, no monitoramento da Usina Hidrelétrica de Ourinhos, nos períodos pré e pós enchimento do reservatório.

A Figura 23, que mostra a similaridade entre as estações de coleta, utilizando-se dados coletados com aspirador elétrico. Novamente as estações Canteiro de Obras e Sítio Caiçara apresentam maior índice de similaridade $(\mathrm{I} j=0,37)$ entre si no período pré, porém é inferior ao obtido com os dados de formas imaturas e sofre redução acentuada no período pós enchimento. O índice entre Canteiro de Obras e Córrego Anhumas é o menor antes da formação do reservatório e aumenta ligeiramente após o enchimento. Já as estações diretamente afetadas não apresentaram alteração expressiva em seu índice.

Nas coletas utilizando-se a armadilha de Shannon as estações Sítio Caiçara e Córrego Anhumas, no período pré enchimento, apresentaram maior similaridade (Fig. 24). Outra observação a ser destacada é que após o enchimento todos os índices 
de similaridade entre as estações de coleta diminuíram, chegando quase a se igualarem.

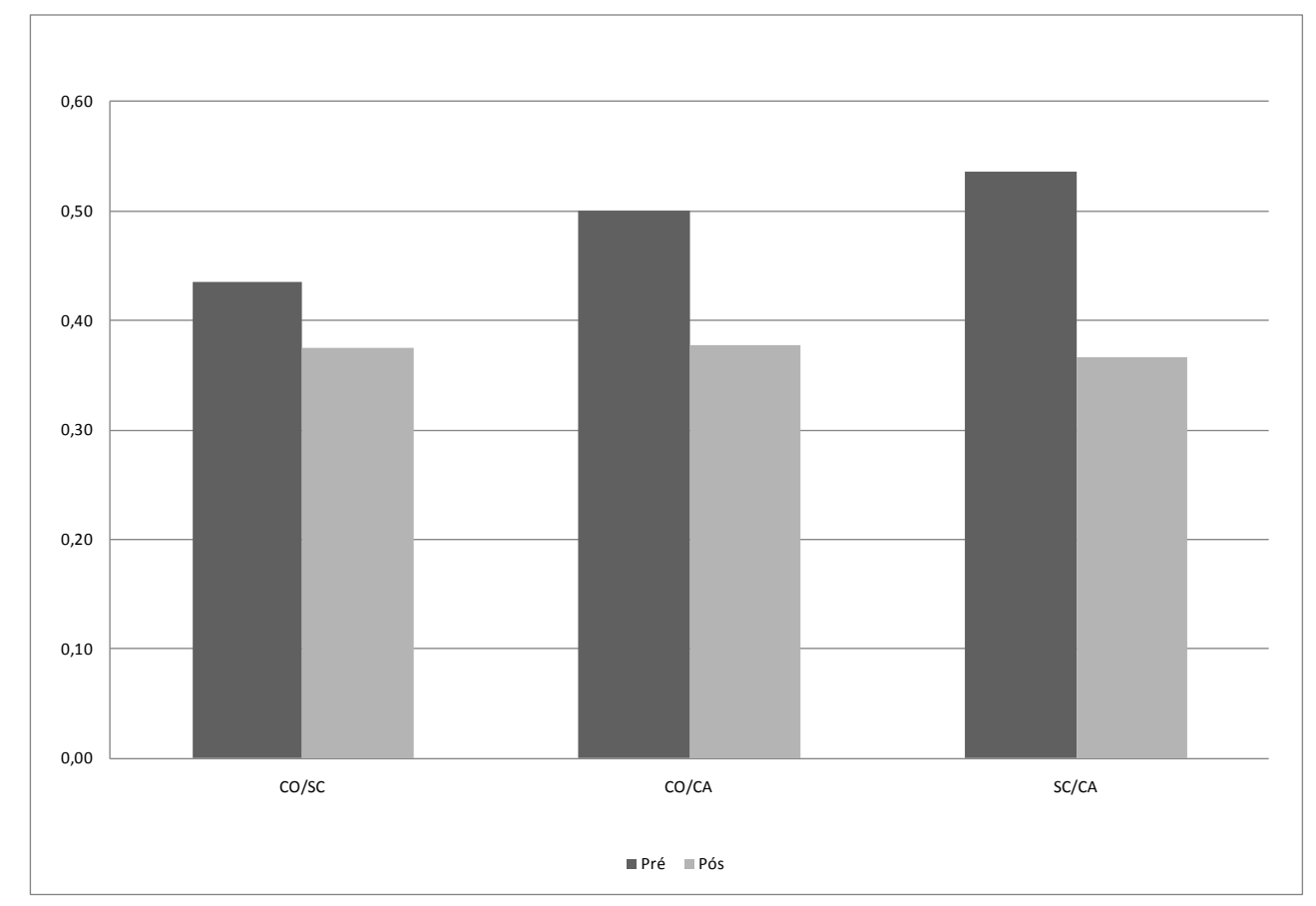

Figura 24. Coeficiente de Similaridade de Jaccard entre estações de coleta, obtida nas coletas com armadilha de Shannon, no monitoramento da Usina Hidrelétrica de Ourinhos, nos períodos pré e pós enchimento do reservatório.

Quando observamos os dados gerais compostos pelos dados obtidos pelas três técnicas de coleta, destaca-se uma maior similaridade entre os pontos de coleta antes do fechamento da barragem e posterior enchimento do reservatório. Sendo que as estações Canteiro de Obras e Sítio Caiçara as que apresentavam maior similaridade. Após o enchimento os índices de similaridade entre o Canteiro de Obras, que não foi afetado diretamente com a formação do reservatório, e as outras estações reduziram, enquanto o índice entre estas não se alterou. 


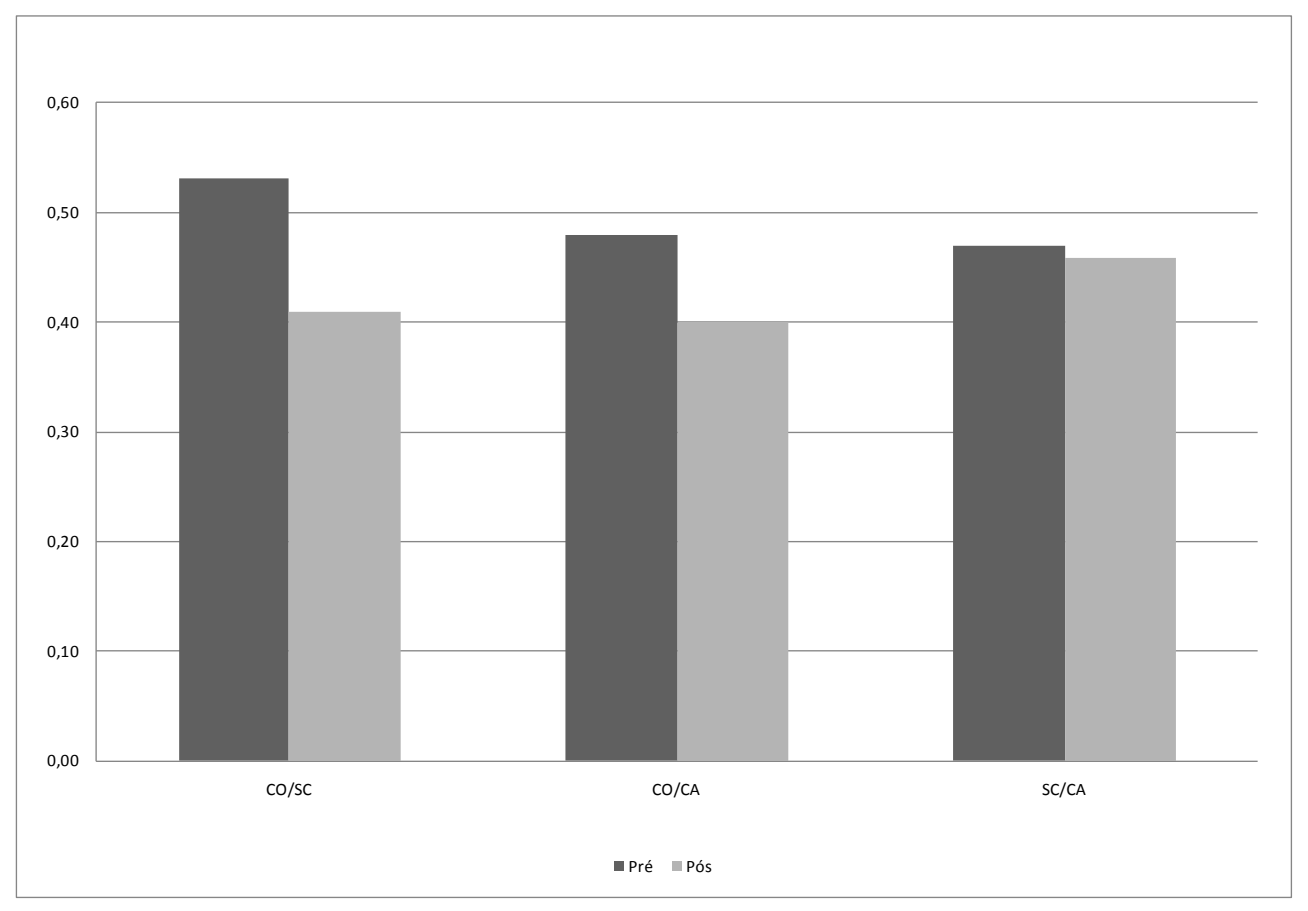

Figura 25. Coeficiente de Similaridade de Jaccard entre estações de coleta, no monitoramento da Usina Hidrelétrica de Ourinhos, nos períodos pré e pós enchimento do reservatório.

\subsubsection{Temporal}

A Figura 26 mostra os coeficientes de similaridade temporal para cada estação de coleta segundo a técnica de coleta empregada. Para as formas imaturas o Canteiro de Obras apresentou maior similaridade entre os períodos pré e pós enchimento e o Sítio Caiçara o menor. Os dados gerados a partir das coletas com aspirador elétrico foram os que apresentaram os menores valores de similaridade, enquanto nas coletas com armadilha de Shannon observam-se os valores mais elevados dentre as três técnicas. Na análise das três técnicas em conjunto (Geral), Canteiro de Obras e Sítio Caiçara são respectivamente as estação que apresentam a maior e a menor similaridade entre os períodos anterior e posterior a formação do reservatório. 


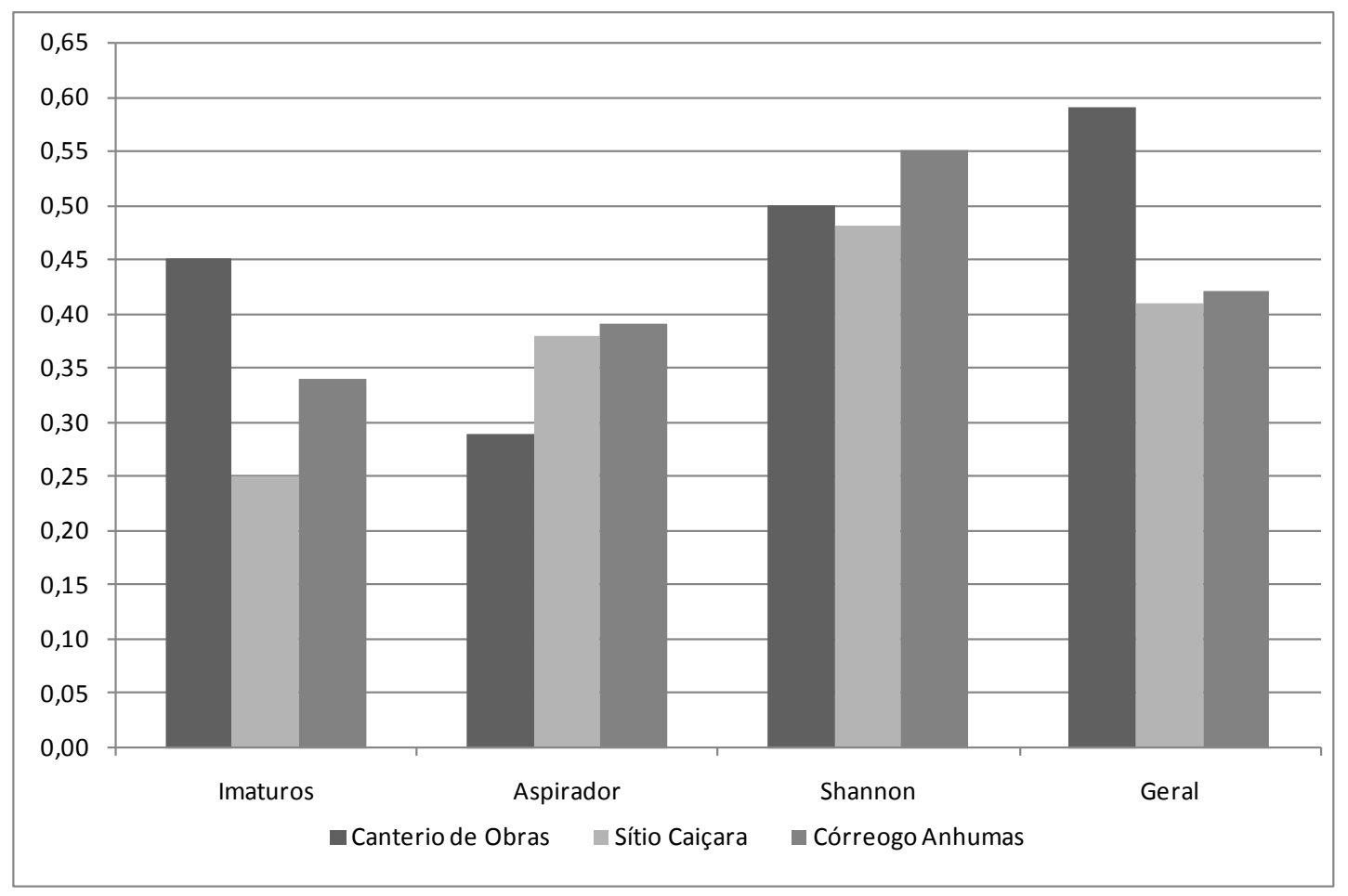

Figura 26. Coeficiente de Similaridade Temporal entre os períodos pré e pós enchimento do reservatório segundo as estações de coleta e técnica de coleta, do monitoramento da Usina Hidrelétrica de Ourinhos, SP. Brasil.

\subsection{Diversidade $\beta$}

Segundo Whittaker (1978), Diversidade $\beta$ a diversidade entre diferentes habitats, que se revela pela heterogeneidade da estrutura da comunidade. Tal heterogeneidade pode refletir-se em dois aspectos principais, a composição das associações de espécies e a proporção entre as abundâncias das diferentes espécies. A Diversidade $\beta$ pode ser calculada com ręđæð ao espaço, ou temporal quando a comunidade é instável apresentando grande fluxo de migração 


\subsubsection{Espacial}

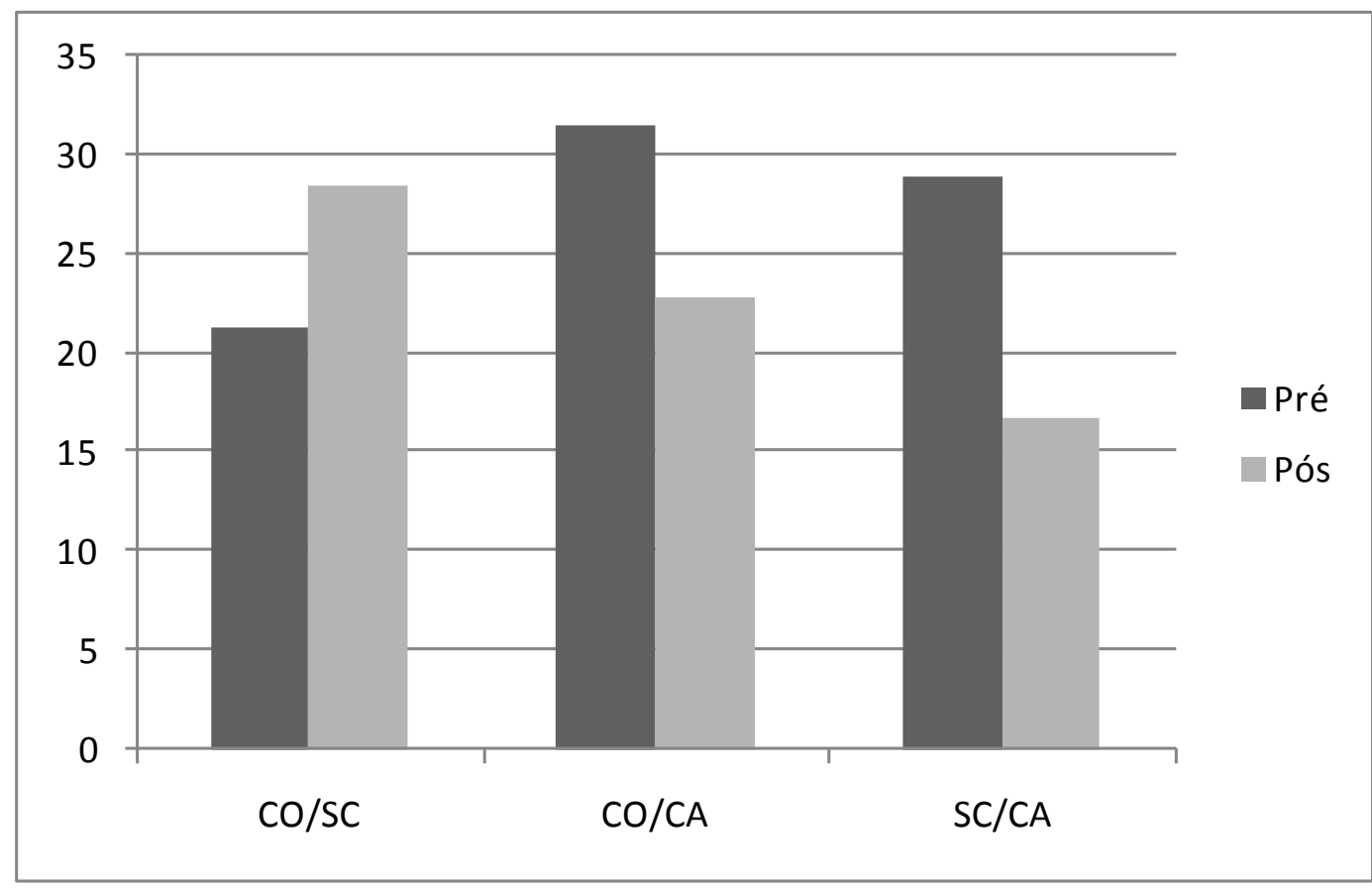

Figura 27. Diversidade $\beta$ entre estações de coleta, obtida nas coletas com concha entomológica para avaliação de formas imaturas, nos períodos pré e pós enchimento do reservatório da Usina Hidrelétrica de Ourinhos.

A diversidade $\beta$ encontrada entre o Canteiro de Obras e o Sítio Caiçara é a menor do período pré enchimento, já no período pós é o que apresenta maior valor. A diversidade $\beta$ diminuiu no período pós para as demais composições (Fig. 27).

A Figura 28 apresenta os dados provenientes das coletas com aspirador elétrico e observam-se tendências similares a da encontradas para formas imaturas, só que os valores de diversidade encontrados foram maiores. O par Canteiro de Obras/Córrego Anhumas apresentou o maior valor nos dois períodos. 


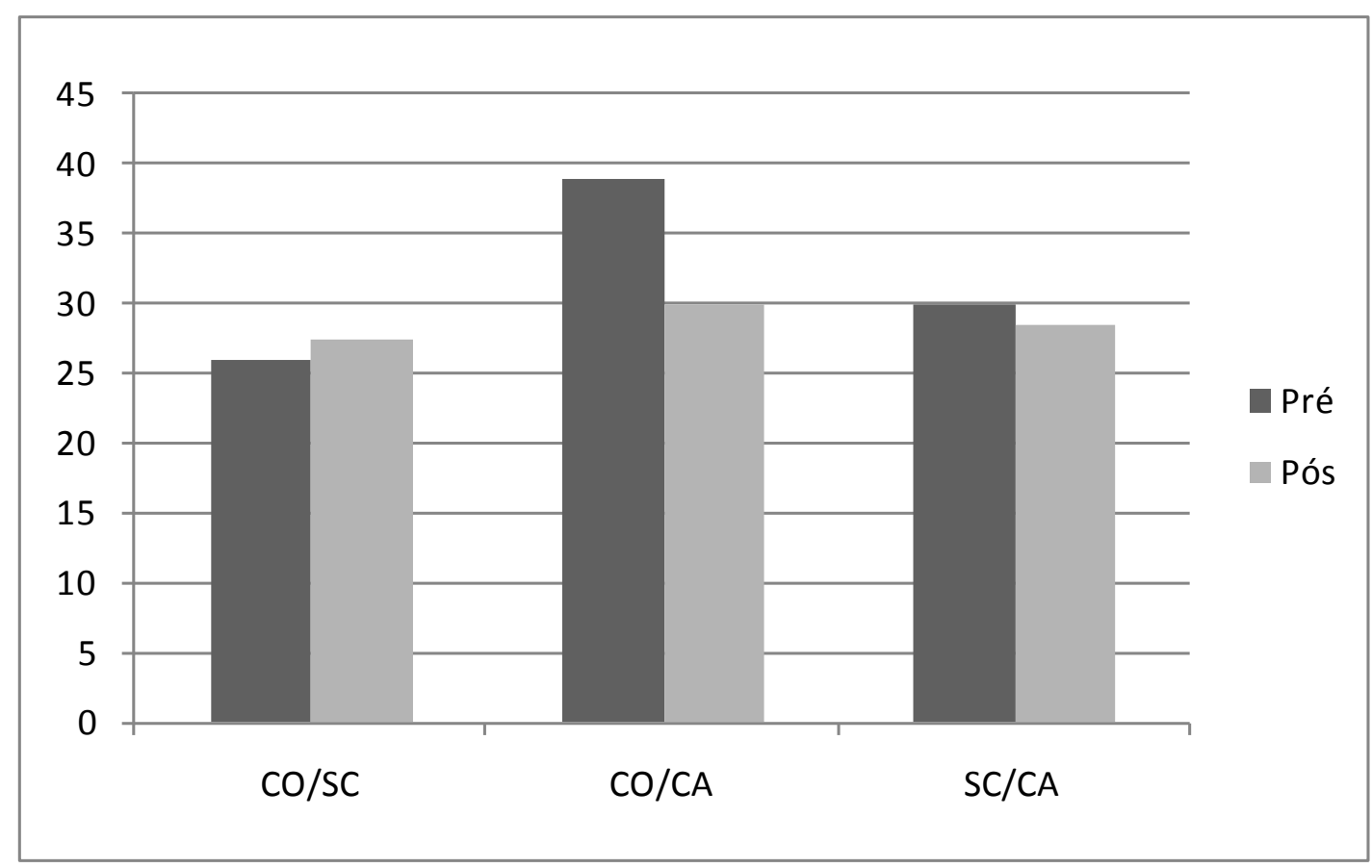

Figura 28. Diversidade $\beta$ entre estçães de coleta, obtida nas coletas com aspirador elétrico, na Usina Hidrelétrica de Ourinhos, nos períodos pré e pós enchimento do reservatório.

A Figura 29 se destaca por apresentar valores maiores no período pós enchimento. E nesse mesmo período observa-se uma maior diversidade no par Canteiro de Obras/Córrego Anhumas. 


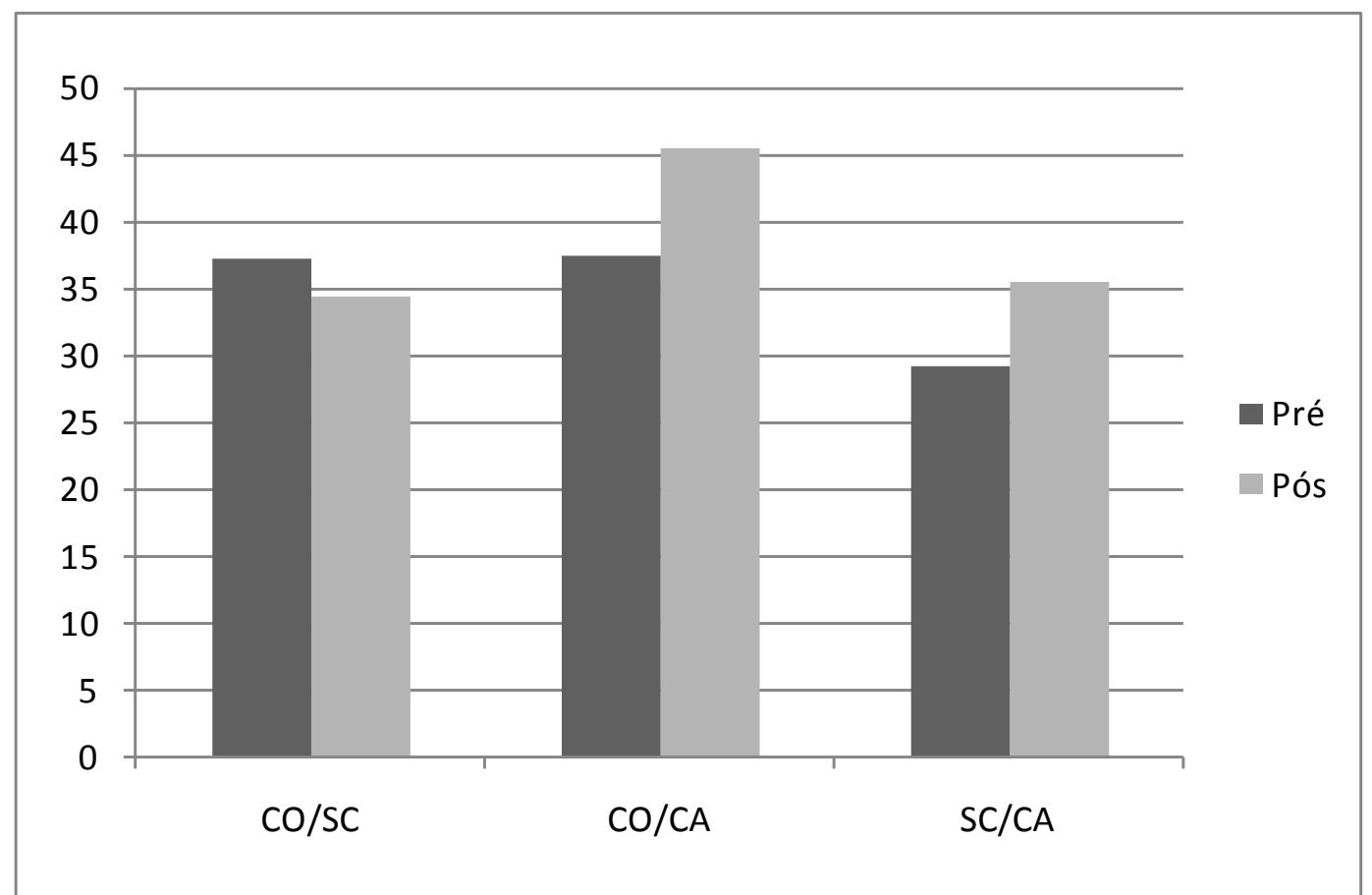

Figura 29. Diversidade $\beta$ entre eșães de coleta , obtida nas coletas com armadilha de Shannon, na Usina Hidrelétrica de Ourinhos, nos períodos pré e pós enchimento do reservatório.

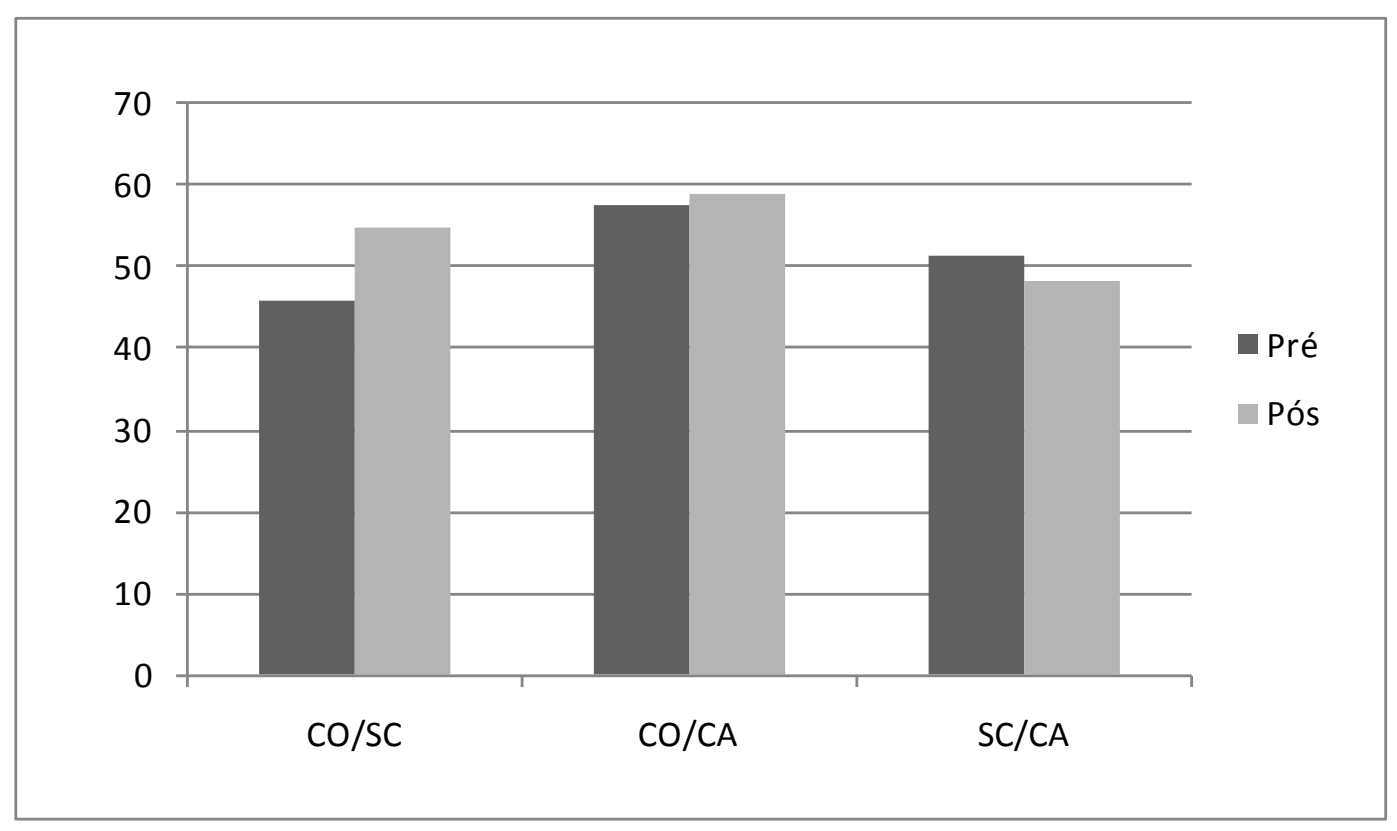

Figura 30. Diversidade $\beta$ entre estações de coleta, obtida utilizando-se as três técnicas de coleta, na Usina Hidrelétrica de Ourinhos, nos períodos pré e pós enchimento do reservatório. 
Quando se observa as diversidades provenientes das três técnicas em conjunto, Canteiro de Obras/Córrego Anhumas mantém a posição de maior valor nos dois períodos, enquanto Canteiro de Obras/Sítio Caiçara e Sítio Caiçara/Córrego Anhumas tem aumento e diminuição da diversidadф̧, respectivamente, no peŕodo pós em relação ao pré (Fig. 30).

\subsubsection{Temporal}

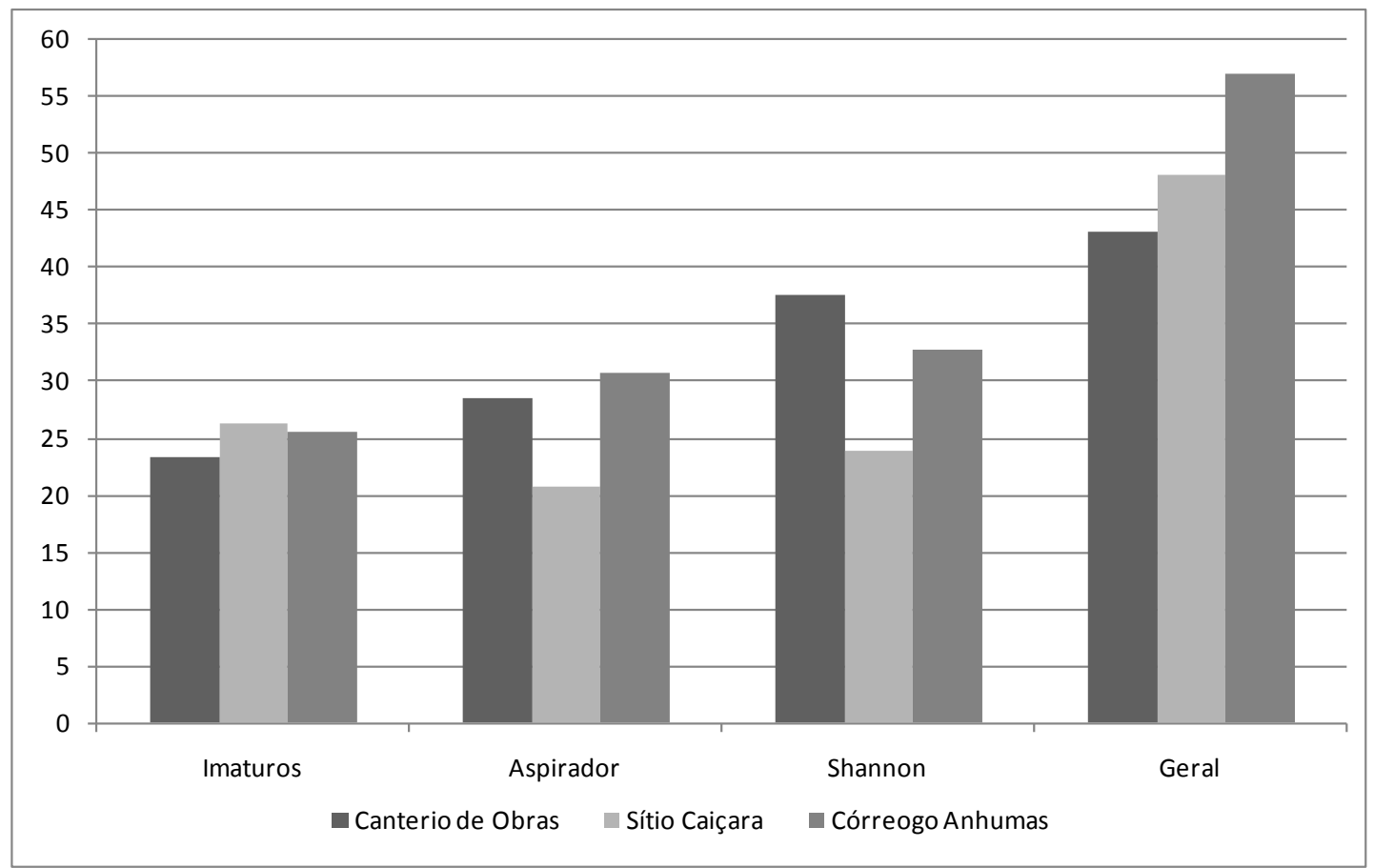

Figura 31. Diversidade $\beta$ Temporal entre os peíodos pré e pós enchimento do reservatório segundo as estações de coleta e técnica de coleta, do monitoramento da Usina Hidrelétrica de Ourinhos.

A Figura 31 mostra a diversidade $\beta$ temporal fór e pós enchimento pra cada estação de coleta segundo a técnica empregada. Sítio Caiçara e Córrego Anhumas apresentam diversidades similares para formas imaturas. Nos dados gerados a partir das coletas com aspirador elétrico, observa-se menor diversidade $\beta$ temporal no Sítio 
Caiçara, seguido pelo Canteiro de Obras, enquanto nas coletas com armadilha de Shannon observa-se que o Canteiro de Obras apresenta o valor mais elevado dentre as três técnicas. Na análise das três técnicas em conjunto (Geral), Córrego Anhumas e Canteiro de Obras são respectivamente as estações que apresentam a maior e a menor diversidade $\beta$ temporal entre os períodos anterior e posterior a formação do reservatório.

\section{Discussão}

Sobre as espécies de importância epidemiológica identificadas em todo o estudo, envolvendo o período pré inundação e o pós inundação destacam-se: Aedes albopictus, Aedes scapularis, Anopheles albitarsis s.l, Anopheles darlingi, gênero Coquillettidia, Culex quinquefasciatus, Culex nigripalpus, subgênero Melanoconion do gênero Culex, Haemagogus leucocelaenus, gênero Mansonia e Psorophora ferox. Dessas, algumas são importantes na veiculação de patógenos como os parasitos da malária e os arbovírus. Entretanto, para que esse risco ocorra, há necessidade desses mosquitos assumirem elevadas abundâncias, fato que apenas ocorreu em certas situações para Aedes scapularis, que possui potencial vetor para arbovírus e Anopheles albitarsis s.l, considerado vetor secundário de plasmódios. Ressalta-se na região a marcante presença dos gêneros Mansonia e Coquillettidia, mosquitos provocadores de incômodo pelas picadas.

No estudo de adultos, em relação ao enfoque da Riqueza nas três estações, mediante as duas técnicas empregadas, nos períodos antes e após a inundação, 
ficaram evidentes que na fase pré-inundação, os valores tenderam a ser ligeiramente mais elevados. Houve queda desse indicador na fase pós-inundação, com recuperação mais acentuada no Córrego Anhumas. Nessa estação esse fenômeno pode ser interpretado, devido às características da paisagem local, antes uma várzea extensa com charcos e vegetação de brejo. Desse local extraiu-se argila na fase anterior à inundação, formando vários pequenos lagos. A seguir foi inundado pelo reservatório de Ourinhos aproximando a margem alagada do sítio de coleta, o que em um primeiro momento causaria a redução do número de espécies. E posteriormente a intensa colonização de macrófitas flutuantes que se proliferou nesse ambiente compondo assim um novo hábitat favorável aos mosquitos. No estudo de formas imaturas, houve nítido aumento da Riqueza na fase pós inundação, porém, esse fato pode ser explicado pelo viés do esforço amostral, ou seja, nessa fase as coletas foram mais produtivas, principalmente nas estações Sítio Caiçaras e Córrego Anhumas, devido ao acesso mais fácil aos criadouros pelo uso de embarcação, além do aumento de macrófitas aquáticas no lago formado.

A análise conjunta dos dados obtidos pelas três técnicas mostrou uma maior riqueza no período pré enchimento para todas as estações de coleta. Já no final do período pós enchimento, do estudo, as estações Canteiro de Obras e Córrego Anhumas sofreram diferentes inflexões, sendo que no primeiro desses locais houve drástica redução do indicador e no segundo aumento, fato que pode ser explicado pelo fato de que mesmo não sofrendo de forma direta os efeitos da formação do lago, o Canteiro de Obras está sob influência do barramento que controla de forma artificial o volume de água do rio, podendo assim impedir que áreas antes inundáveis não sejam mais. Já no caso do Córrego Anhumas, assim como do Sítio Caiçara a 
formação do lago destrói determinados tipos de criadouros, mas com o processo de sucessão ecológica, novos nichos são criados, sendo propícios ao desenvolvimento de mosquitos.

As curvas de rarefação calculadas pelo software Ecosim 7.0 (Gotelli e Entsminger, 2009) mostram que quando igualamos o tamanho das amostras, as riquezas dos períodos pré e pós do Canteiro de Obras e Córrego Anhumas não sofrem modificação significativa $(\mathrm{p}<95 \%)$, pois em ambos os casos a diferença entre as riquezas encontradas em cada período seriam resultado do tamanho maior da amostra no período pré.

O Sítio Caiçara apresentou em sua curva de rarefação uma riqueza no período pós superior ao do período pré, podendo ser resultado das alterações causadas pelo empreendimento, como a aproximação dos criadouros à fonte de repasto e ao ponto de coleta.

Em relação ao estudo da Diversidade $\alpha$, nas três técnicas houve redução da diversidade calculada pelo índice de Margalef no Canteiro de Obras o que pode ser resultado de alterações no micro clima ou devido a própria atividade humana desenvolvida no local.

Os dados gerais mostram apenas aumento da diversidade no Sítio Caiçara o que, como já foi comentado antes, pode ser efeito da utilização da embarcação para as coletas de formas imaturas no período pós, sendo também associado a diversificação dos criadouros, presença de macrófitas, e proximidade destes com locais de repouso e repasto para os culicídeos.

A dominância, outro componente utilizado no estudo de diversidade $\alpha$, a dominância tem como principio mostrar qual a probabilidade de que o próximo 
indivíduo coletado na amostra seja da mesma espécie que o anterior. Os valores de dominância no Canteiro de Obras, como esperado, não sofreram variações drásticas, mas isso não descarta alterações na composição de sua fauna. As reduções das dominâncias no Sítio Caiçara e no Córrego Anhumas são explicadas pelos fatores já citados anteriormente.

No estudo do Índice de Abundância de Espécies Padronizadas (IAEP) houve tendência à redução do número de espécie com o impacto da inundação, com exceção nas coletas de formas imaturas na fase pós-enchimento, que foram facilitadas mediante o uso da embarcação, no Sítio Caiçara. Em várias situações, as espécies mais abundantes, isto é, aquelas com IAEPs mais elevados, permaneceram como as primeiras nos “rankings” após a inundação, porém, nessa fase os valores dos IAEPs foram menores. Destaca-se o surgimento de uma importante espécie, o Anopheles darlingi, sempre ausente no período pré-enchimento, porém, presente na fase inundada. Essa espécie apareceu em campanhas realizadas na fase do lago preenchido nas estações do Sítio Caiçara e Córrego Anhumas, associadas diretamente à represa, em algumas coletas com aspirador, armadilha de Shannon e com concha entomológica, porém os IAEPs chegaram ao máximo a 0,18, significando baixa abundância. Outra espécie que merece destaque foi Anopheles albitarsis s.l, que nas estações próximas do lago ganhou posições mais elevadas no “ranking”, chegando a $1^{\mathrm{a}}$ posição na estação Sítio Caiçara e a $2^{\mathrm{a}}$ posição na estação Córrego Anhumas, aumentou o valor do IAEP, tendo alcançado valor consideravelmente elevado (0,66 no Sítio Caiçara e 0,90 no Córrego Anhumas), retratando elevada abundância. Esses resultados denotam certa tendência de adaptação de anofelinos Nyssorhynchus de forma a aferirem vantagens com a 
formação do reservatório. As espécies de anofelinos comentadas são consideradas respectivamente como vetor primário e secundário da malária no Brasil.

Dentre as espécies que apresentaram maiores índices de abundância durante o período de estudo as que se destacam por sua importância epidemiológica:

Anopheles albitarsis s.l é considerado um complexo cujos membros ainda não estão devidamente separados morfologicamente (Wilkerson e col. 2005). Por isso, nesse estudo, tratamos An. albitarsis s.l como uma única espécie. Ele é considerado vetor secundário ou, no máximo, local de $P$. vivax e $P$. falciparum (Consoli e Lourenço-de-Oliveira, 1994).

O Ae. (Och.) scapularis que foi considerado como suspeito de transmitir o vírus Rocio da encefalite, que causou epidemia na Baixada Santista e Vale do Ribeira, no período de 1975 a 1978 (Iversson, 1988). E também é citado como vetor de outros arbovírus (Consoli e Lourenço-de-Oliveira, 1994).

$C x$. (Cux.) coronator tem sido encontrado infectado por arbovírus causadores de encefalites ou de doenças febris (Forattini, 2002).

As espécies dos gêneros Mansonia e Coquillettidea não são vetoras de doenças endêmicas no Brasil. Porém esses gêneros representam problema para os moradores das áreas próximas aos seus criadouros, pois são geralmente numerosas e antropofílicas.

Ao compararmos os índices de similaridade entre os pontos de coleta para cada período percebemos que as diferentes técnicas apresentaram padrões que são explicados pelos vieses amostrais presente nelas. As formas imaturas no período pré Canteiro de Obras e Sítio Caiçara apresentaram maior similaridade entre esses, devido a distância do leito do rio para o ponto de coleta, já no período pós esse 
quadro se altera e Sítio Caiçara e Córrego Anhumas apresentam similaridade superior a 50\% justificável pela formação do reservatório e as coletas realizadas nele. A armadilha de Shannon apresentou no período pós, similaridades próximas a $40 \%$ entre os três pontos de coleta, explicado pelo fato de se tratar de uma técnica atrativa passiva e de longo alcance. Ao analisarmos os dados gerais minimizamos o viés presente em cada técnica de amostragem, observamos que as estações mais próximas apresentam maior similaridade e o Canteiro de Obras tem redução de sua similaridade com as outras estações, onde houve a formação do reservatório e entre os pontos dessa área a similaridade aumenta.

Na literatura de culicídeos são raros os trabalhos de monitoramento que calculam o índice de similaridade temporal que pode servir como um parâmetro para o grau de modificação que a fauna de culicídeos sofreu. Sendo assim, observa-se que as estações em que o reservatório foi formado apresentam menor similaridade entre os períodos pré e pós, sendo o fruto da redução de espécies causado pela formação do lago e/ou pela substituição de espécies provocada pela alteração do ambiente.

A distância entre as estações amostrais pode explicar os valores de diversidade $\beta$ encontrados, pois se observa uma diversidade maior estimada para o par Canteiro de Obras/Córrego Anhumas em todas as técnicas de coleta, no período pré, e no período pós somente a coleta de formas imaturas é que apresentou maior diversidade $\beta$ para Canteiro de Obras/\$tio Caiçara (28,39 espécies), resultado esse causado pela alteração nos corpos d’água.

Quando agrupamos os dados das diferentes técnicas, reduzindo assim o efeito do viés de cada uma, então se observa que Canteiro de Obras/Córrego Anhumas é a combinação que apresenta a maior diversidade $\beta$, após o enchimento do reservatório, 
Canteiro de Obras/Sítio Caiçara teve um aumento em sua diversidade $\beta$ resultado da alteração na composição da fauna do Sítio Caiçara, e quando vemos a diversidade de Sítio Caiçara/Córrego Anhumas tem-se uma redução devido a maior homogeneização do meio.

Observa-se que o enchimento do reservatório modificou a composição faunística dos pontos de coleta e isso é evidenciado principalmente pelos altos valores de diversidade $\beta$ temporal nas estações, Sítio Caiçara (48,07 espécies) e Córrego Anhumas $(56,81)$, diretamente por ele afetada

\section{Conclusão}

Como conclusão geral, depreende-se que o lago formado pela represa de Ourinhos alterou a fauna de culícideos do local. Observam-se variações em todas as estações de coleta com diferentes gradações relacionadas com o impacto da inundação. Houve alterações principalmente na composição da fauna, ou seja, na diversidade $\alpha$ estrutural, causada por variações na riqueza e “realocação” de espécies. Outra alteração que devemos destacar é com relação à abundância das espécies, que com a intervenção antrópica, alterou os hábitats já existentes, fazendo com que espécies raras ou ausentes viessem a ocupar locais de destaque na composição da fauna de culicídeos.

Os índices de Similaridade e a Diversidade $\beta$ comprovam os efeitos da alteração, pois mostram que a composição da fauna de culicídeos modificou-se com o impacto, tornando o antes e o depois distintos, e sugerindo um grau de 
homogeneização das estações de coleta diretamente circunvizinhas à área do reservatório.

Admite-se que o lago ainda esteja em processo de sucessão ecológica e que a fauna de mosquitos no período de operação da Usina tenderá a se estabilizar nos próximos anos, o que implicaria em estudos de monitoramento de longo prazo.

Dentre os vetores identificados o Anopheles (Nys.) darlingi e o Anopheles albitarsis s.l, pelo papel que desempenham na epidemiologia da malária, merecem atenção. Porém, o risco de transmissão de plasmódio no entorno do lago parece remoto devido à baixa densidade populacional humana, nesse espaço geográfico. Convém ressaltar que o An. darlingi, considerado o principal vetor de plasmódio no Brasil, foi detectado apenas no período pós impacto e em baixíssima densidade, incompatível com a transmissão.

Outras espécies de importância epidemiológica que apresentaram destaque devido à sua abundância e que se beneficiaram com as alterações geradas pelo empreendimento foram $C x$. (Cux.) coronator, vetor de arbovírus, e os gêneros Mansonia e Coquillettidea, causadores de incomodo por sua voracidade.

O Ae. (Och.) scapularis, vetor de arbovírus, muito abundante no período pré e consequentemente apresentando importância epidemiológica, com as alterações sofreu declínio, reduzindo assim o risco de contato com a população humana e transmissão de patógenos. 


\section{Referências}

Bermann C. Impasse e controvérsias da hidreletricidade. Estudos Avançados 2007; 21 (59): 139-53.

Consoli RAGB, Lorenço de Oliveira R. Principais mosquitos de importância sanitária no Brasil. Rio de Janeiro: FIOCRUZ, 1994.

Consolim J, Luz E, Pellegrini NJM, Torres PB. O Anopheles (Nyssorhynchus) darlingi ROOT, 1926 e a malária no lago de Itaipu, Estado do Paraná, Brasil: Uma revisão de dados (Díptera, Culicidae). Arq. Biol. Tecnol. 1991, 34 (2): 263 - 86.

Correa RR, Ramos AS, Rachou RG. Anofelismo e malária em algumas represas do Estado de São Paulo. Rev bras Malar, 1966; 18:455-463.

Dorvillé L. F. M. Mosquitoes as bioindicators of forest degradation in southeastern Brazil, a statistical evaluation of published data in the literature. Stud Neotrop Environ 1996; 31: 68-78.

Falavigna-Guilherme AL, Silva AM, Guilherme EV, Morais DL. Retrospective study of malaria prevalence and Anopheles genus in the area of influence of the Binational Itaipu Reservoir. Rev Inst Med Trop, 2005; (42):81-86

Ferrete JA, Lemos JC, Lima SC. Lagos artificiais e os fatores condicionantes e determinantes no processo saúde - doença. Caminhos de Geografia, 2004; 5(13): $187-200$

Forattini OP, Gomes AC, Natal D, Santos JL. The activity of Culicidae mosquitoes in plain forest and an epidemiological profile of several environments in the Ribeira Valley, São Paulo, Brazil. Rev Saúde Pública 1986, 20(3): 178203.

Forattini OP, Massad E. Culicidae vectors and antropic changes in Southern Brazil natural ecosystem. Ecosystem Health. 1998; 4: 9-19.

Forattini OP. Culicidologia Médica. São Paulo: Edusp; 2002. v.2.

Goldemberg J, Lucon O. Energia e meio ambiente. Estudos Avançados 2007; 21 (59): 7-20.

Gomes AC, Natal D, Paula MB, Urbinatti PR, Mucci LF, Bitencourt MD. Riqueza e abundância de Culicidae (Diptera) em área impactada, Mato Grosso do Sul, Brasil. Rev Saúde Pública 2007, 41(4): 661-4.

Gotelli, N.J. and G.L. Entsminger. 2009. EcoSim: Null models software for ecology. Version 7. Acquired Intelligence Inc. \& Kesey-Bear. Jericho, VT 05465. http://garyentsminger.com/ecosim.htm

Guimarães AE, Gentile C, Alencar J, Lopes CM, Mello RP. Ecology of Anopheline (Diptera, Culicidae), malaria vectors around the Serra da Mesa Reservoir, State of Goiás, Brazil. I - Frequency and climatic factors. Cad Saúde Pública 2004, 20(1): 291-302. 
Guimarães AE, Mello RP, Lopes CM, Alencar J, Gentile C. Prevalência de Anofelinos (Diptera: Culicidae) no crepúsculo vespertino em áreas da Usina Hidrelétrica de Itaipu, no Município de Guaíra, Estado do Paraná, Brasil. Mem Inst Oswaldo Cruz. 1997; 92:745-54.

Iversson LB. Aspectos da epidemia de encefalite por arbovírus na região do Vale do Ribeira, S. Paulo, Brasil, no período de 1975 a 1978. Rev. Saúde Pública, 1980, 14: 9-35.

Karabastos N. Ed. International catalogue of arboviruses including certain other viruses of vertebrates. $3^{\text {nd }}$ ed. San Antonio, Texas, Am Soc Trop Med Hyg, 1985 (Suppl. 1991).

Krebs C.J. Ecology: The experimental analysis of distribution and abundance. 2.ed. New York, Harper \& Row, 678p. 1978

Lane J. Neotropical culicidae. São Paulo: Ed. Universidade de São Paulo: 1953. v.2.

Luna EJA, Pereira LE, Souza RP de. Encefalite do Nilo Ocidental, nossa próxima epidemia? Epidemiol Serv Saúde 2003; 12(1): 7-19.

Luz SL, Lourenço-de-Oliveira R. Forest Culicinae mosquitoes in the environs of Samuel Hydroeletric plant, State of Rondonia, Brazil. Mem Inst Oswaldo Cruz 1996, 91(4): 427-32.

Magurran AE. Ecological diversity and its measurement. Princeton NJ: Princeton University Press. 1988

Magurran AE. Measuring Biological Diversity. Blackwell Publishing. 2004

Marcondes C.B; Fernandes A. and Muller GA Mosquitos (Diptera:Culicidae) nos arredores de uma represa no oeste do estado brasileiro sulino de Santa Catarina, Brasil. Biota Neotrop. 2006. 6(3):

Martins FR, Pereira EB, Echer MPS. Levantamento dos recursos de energia solar no Brasil com o emprego de satélite geoestacionário: o Projeto Swera. Rev. Bras. Ens. Fis., 2004, 26(2):145-59.

Monosowski E. O sertão vai virar mar... Avaliação e gestão ambiental na barragem de Tucuruí, Amazônia. In: Ab’Saber AN \& Müller-Platenberg C. Previsão de impactos: $O$ estudo de impacto ambiental no Leste, Oeste e Sul. Experiências no Brasil, na Rússia e na Alemanha. Ed. Universidade de São Paulo, 2006 (2ª . edição, $2^{\mathrm{a}}$. reimpressão: 2006). 573p.

Morales MA, Barrandeguy M, Fabbri C, Garcia JB, Vissani A, Trono K, Gutierrez G, Pigretti S, Menchaca H, Garrido N, Taylor N, Fernandez F, Levis S, Enría D. West Nile Virus isolation from equines in Argentina, 2006. Emerging Infectious Diseases 2006, 12(10): 1559-1561.

Müller AC. Hidrelétricas, meio ambiente e desenvolvimento. São Paulo: Makron Books do Brasil; 1995.

Nasci RS. A light weight batterypowered aspirator for collecting resting mosquitoes in the field. Mosq News 1981, 41: 808-11. 
Natal D. Efeitos da inundação sobre culicídeos, com ênfase na população de Aedes scapularis Rondani, 1848 da área de influência da Hidrelétrica de Porto Primavera. [tese de Livre-Docência, Departamento de Epidemiologia, FSP/USP] São Paulo, 2001.

Natal D, Araújo FAA, Vianna RST, Pereira LE, Ueno HM. O mosquito das águas poluídas. Saneas 2004, 2(19): 26-31.

Natal D, Barata EAMF, Urbinatti P, Barata JMS. Contribuição ao conhecimento da fauna de imaturos de mosquitos (Diptera, Culicidae) em área de implantação de hidrelétrica na bacia do Rio Paraná, Brasil. Rev Bras Entomol, 1995; 39:897-9.

Natal D, Barata EAMF, Urbinatti P, Barata JMS, Paula MB. Sobre a fauna de mosquitos adultos (Diptera, Culicidae) em área de implantação de hidrelétrica na bacia do Rio Paraná, Brasil. Rev Bras Entomol. 1998; 41:213-6

Natal D, Paganelli CH, Santos JLF. Composição da população adulta de Culex (Culex) quinquefasciatus Say, 1823 em ecótopos próximos à represa Edgard de Souza, no município de Santana do Parnaíba, estado de São Paulo, Brasil. Rev Bras Entomol 1991, 35(3): 539-543.

Natal D, Ueno HM. Vírus do Nilo Ocidental: características da transmissão e implicações vetoras. Entomol. Vect. 2004, 11(3): 417-433, 2004.

Odum E. Ecologia.Rio de Janeiro: Ed. Guanabara; 1988.

Paula MB, Gomes AC. Culicidae (Diptera) em área sob influência de construção de represa no Estado de São Paulo. Rev Saúde Pública 2007, 41(2): 284-9.

Quintero LO, Thatcher BD, Tadei WP. Biologia de anofelinos amazônicos. XXI. Ocorrência de espécies de Anopheles e outros culícideos na área de influência da hidrelétrica de Balbina - cinco anos após o enchimento do reservatório. Acta Amazonica. 1996, 26 (4): 281-296.

R Development Core Team (2007). R: A language and environment for statistical computing. R Foundation for Statistical Computing, Vienna, Austria. ISBN 3-900051-07-0, URL http://www.R-project.org

Roberts DR, Hsi BP. An index of species abundance for use with mosquito surveillance data. Environ Entomol. 1979; 8: 1007-13.

Schmidt CAJ, Lima MAM. A demanda por energia elétrica no Brasil. Rev. Bras. Econ. 2004, 58(1) : 68-98.

Service MW. Mosquito ecology: field sampling method. $2^{\text {nd }}$. ed. London: Applied Science Publ; 1993.

Shannon R. Methods for collecting and feeding mosquitoes in jungle yellow fever studies. Am J Trop Med Hyg 1939, 19: 131-40.

Southwood TRE. Ecological methods. $2^{\text {nd }}$ ed. London: Chapman and Hall, 1978. 
Tadei WP, Thatcher BD, Santos JM, Scarpassa VM, Rodrigues IB, Rafael MS. Ecologic observations on anopheline vectors of malaria in the Brazilian Amazon. Am J Trop Med Hyg 1998, 52(9): 325- 35.

Thiengo SC, Santos SB, Fernandez MA. Malacofauna límnica da área de influência do lago da usina hidrelétrica de Serra da Mesa, Goiás, Brasil.: I. Estudo qualitativo. Rev. Bras. Zool. 2005, 22(4): 867-74.

Tundisi JG. Exploração do potencial hidrelétrico da Amazônia. Estudos Avançados 2007; 21 (59): 109-17.

Turrel MJ, Sardelis MR, Dohm DJ, O’Guinn ML. Potencial North American vectors of West Nile Virus. Ann N Y Acad Sci 2001; 951: 317-24.

Vainer C B. Recursos hidráulicos: questões sociais e ambientais. Estudos Avançados 2007; 21 (59): 119-37.

Vittor AY, Gilman RH, Tielsch J, Glass G, Shieds T, Lozano WS, Cancino PV, Patz JA. The effect of deforestation on the human-bitting rate of Anopheles darlingi, the primary vector of falciparum malaria in the Peruvian Amazon. Am J Trop Med Hyg 2006, 74(1): 3-11.

Whittaker, RH. Evolution and measurement of species diversity. Taxon, v. 21, p. 213-251, 1972.

Wilkerson RC, Foster PG, Li C, Sallum MA. Molecular Phylogeny of Neotropical Anopheles (Nyssorhynchus) Albitarsis Species Complex (Diptera: Culicidae). Ann Entomol Soc Am. 2005 Nov;98(6):918-925 
Anexos 
Anexo I - Área de estudo e estações de coleta de mosquitos com indicações das áreas amostradas

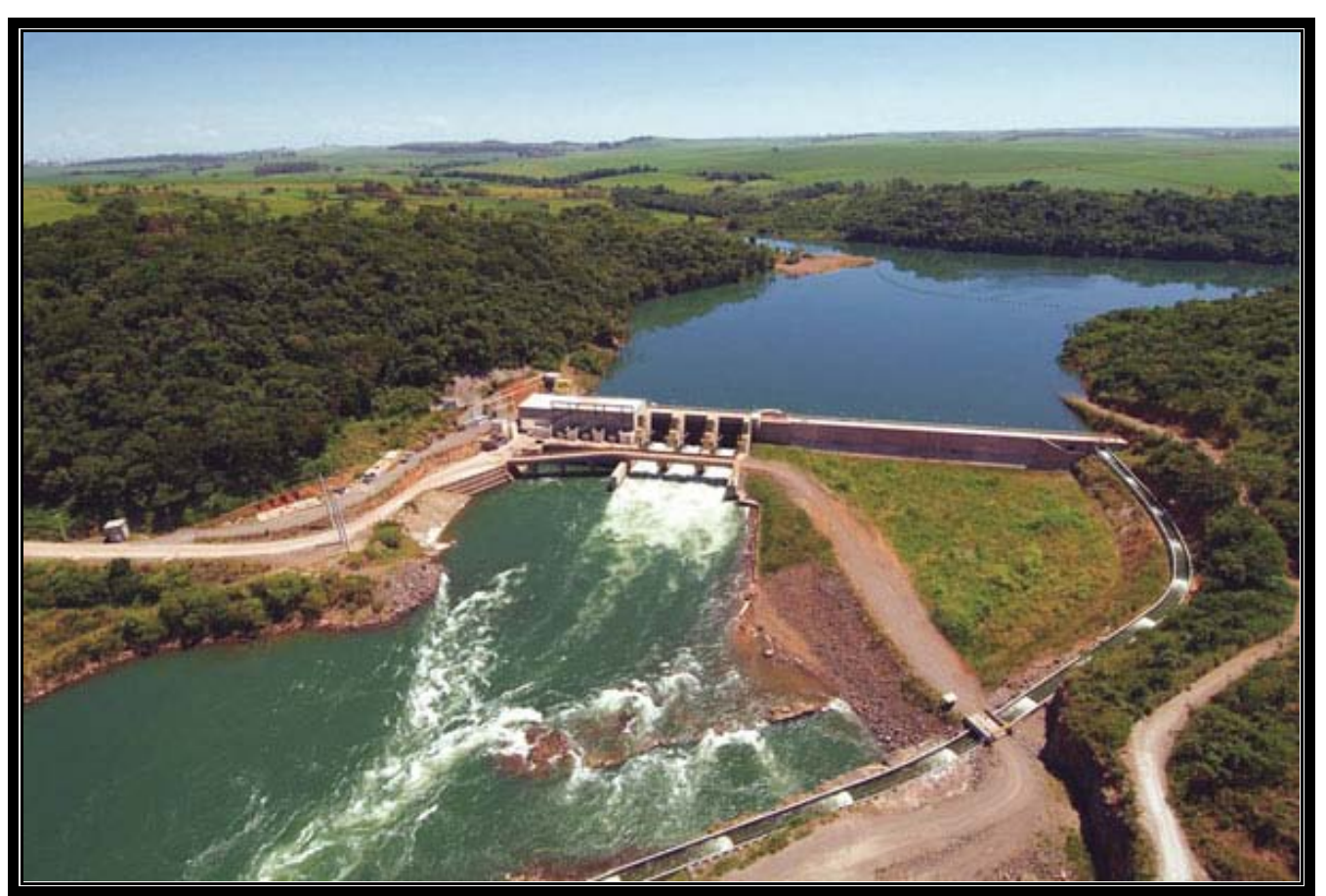

Fonte: CBA

Figura 32. Hidrelétrica Ourinhos - Vista Aérea

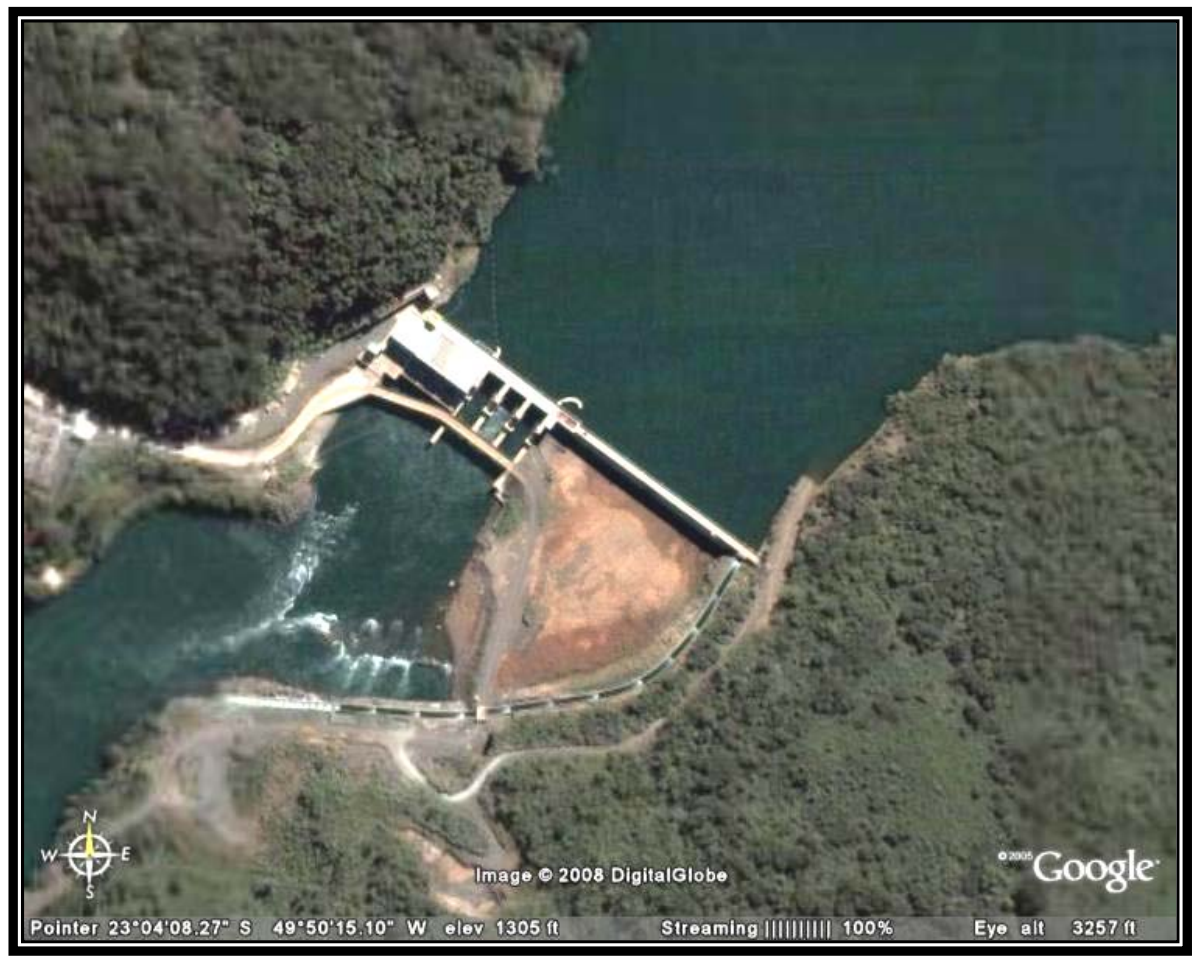

Figura 33. Hidrelétrica Ourinhos - Vista por satélite 


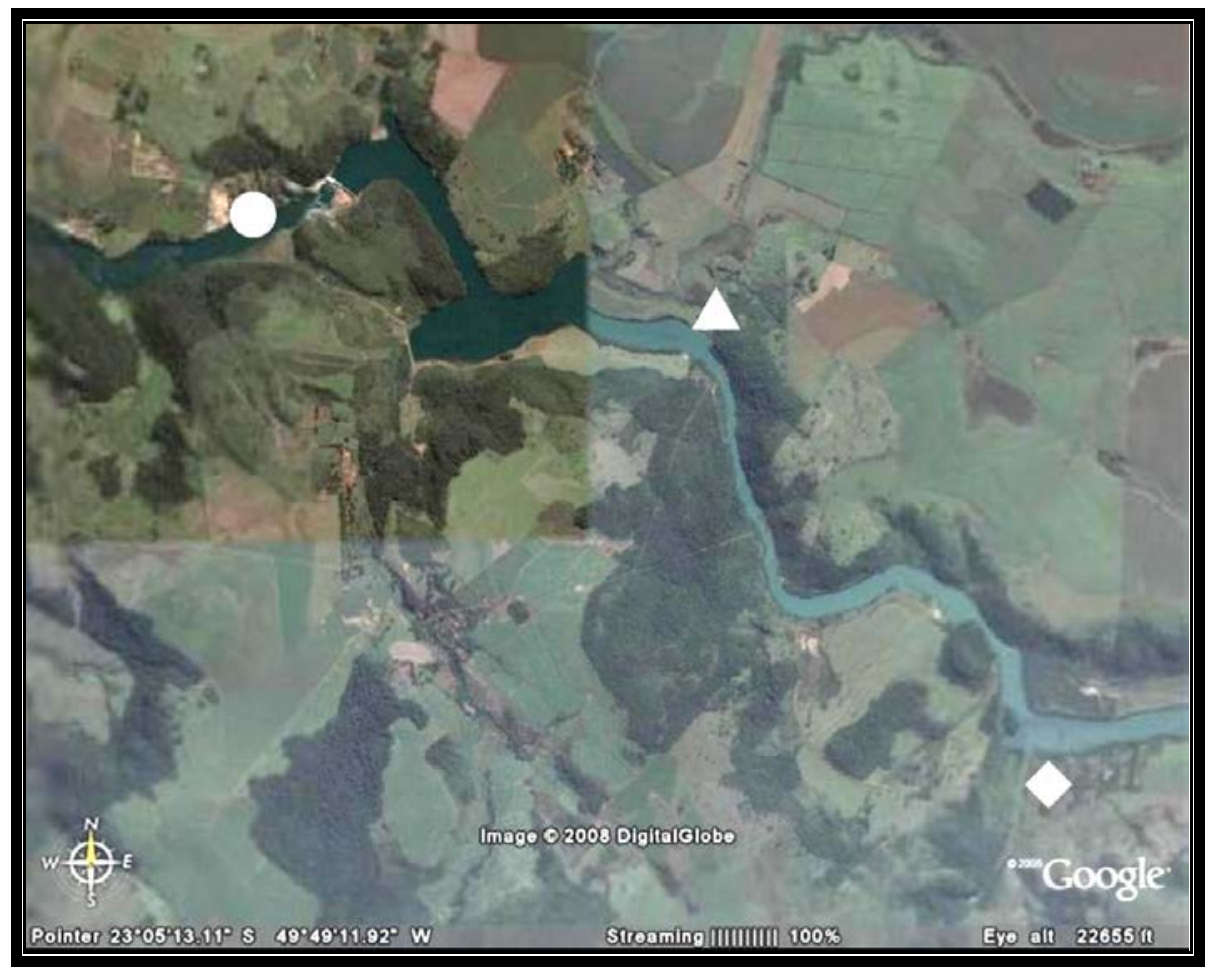

Figura 34. Localização das estações de coleta - Vista por satélite. Círculo - Canteiro de obras; Triângulo - Sítio Caiçara; Losângulo - Córrego Anhumas

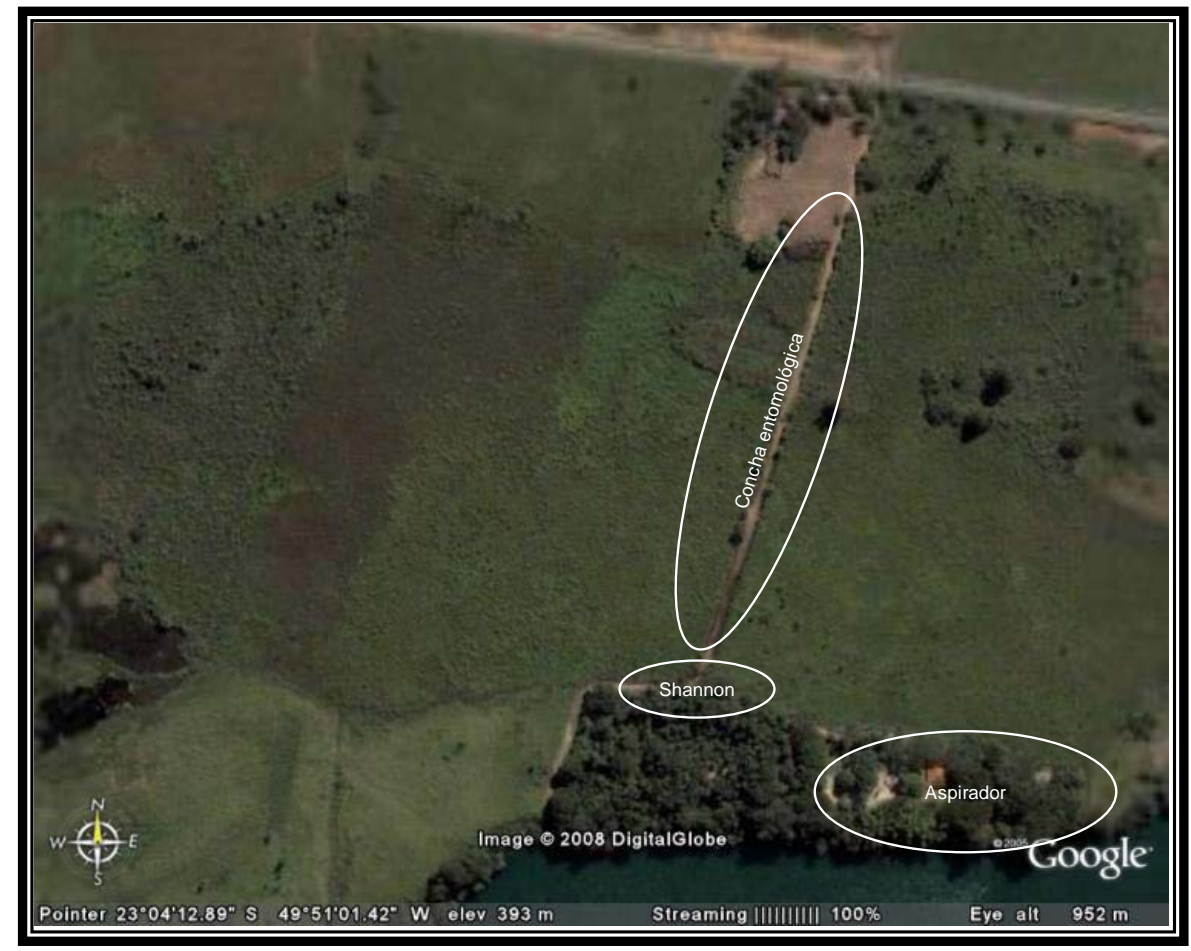

Figura 35. Estação de coleta de culicídeos Canteiro de Obras - localizada na margem direita do Rio Paranapanema, no município de Ourinhos, nas proximidades do eixo da barragem, à jusante. São mostradas as áreas onde foram feitas as coletas de mosquitos adultos com armadilha de Shannon, com aspirador elétrico e coletas de formas imaturas com concha entomológica. 


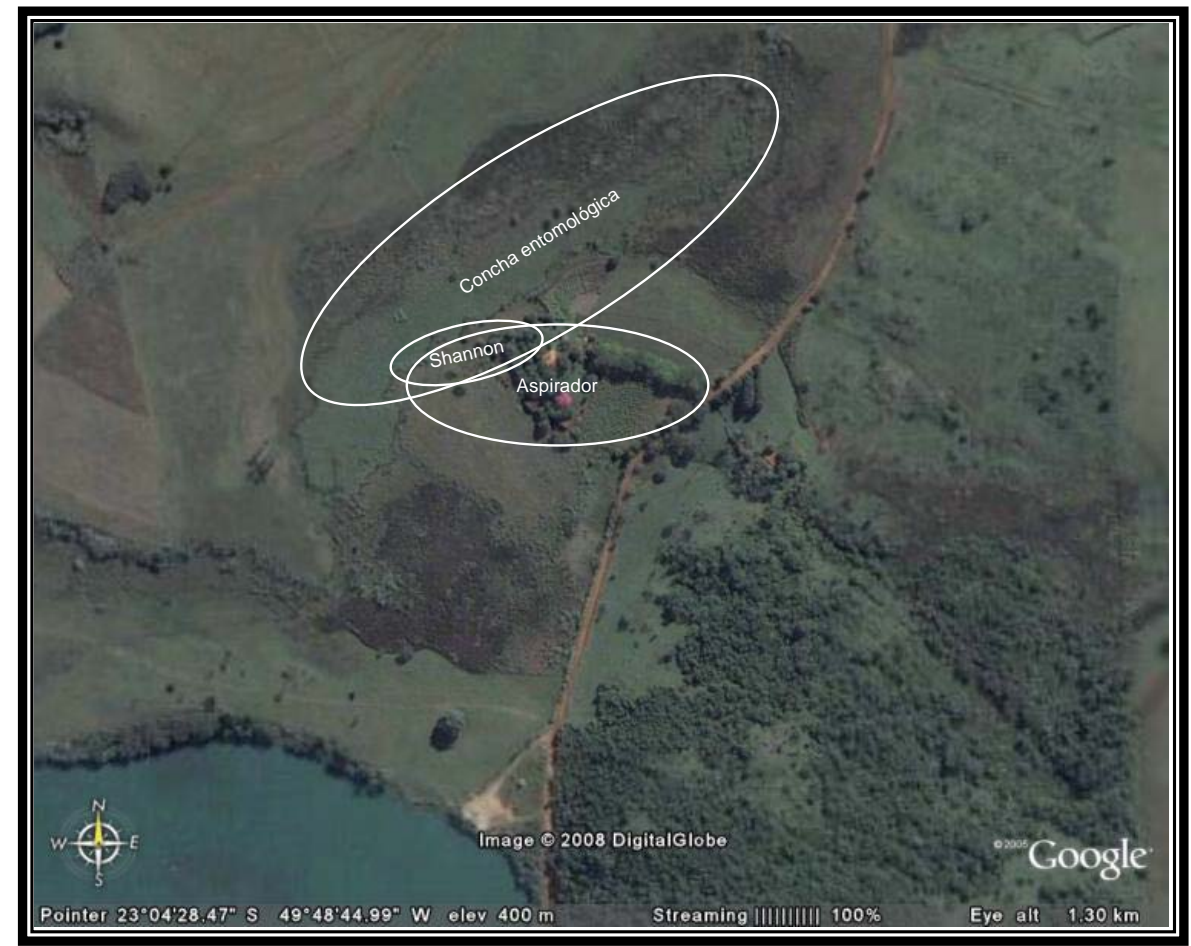

Figura 36. Estação de coleta de culicídeos Sítio Caiçara - localizada na margem direita do Rio Paranapanema, no município de Chavantes, SP. São mostradas as áreas onde foram feitas as coletas de mosquitos com armadilha de Shannon, com aspirador elétrico e com concha entomológica.

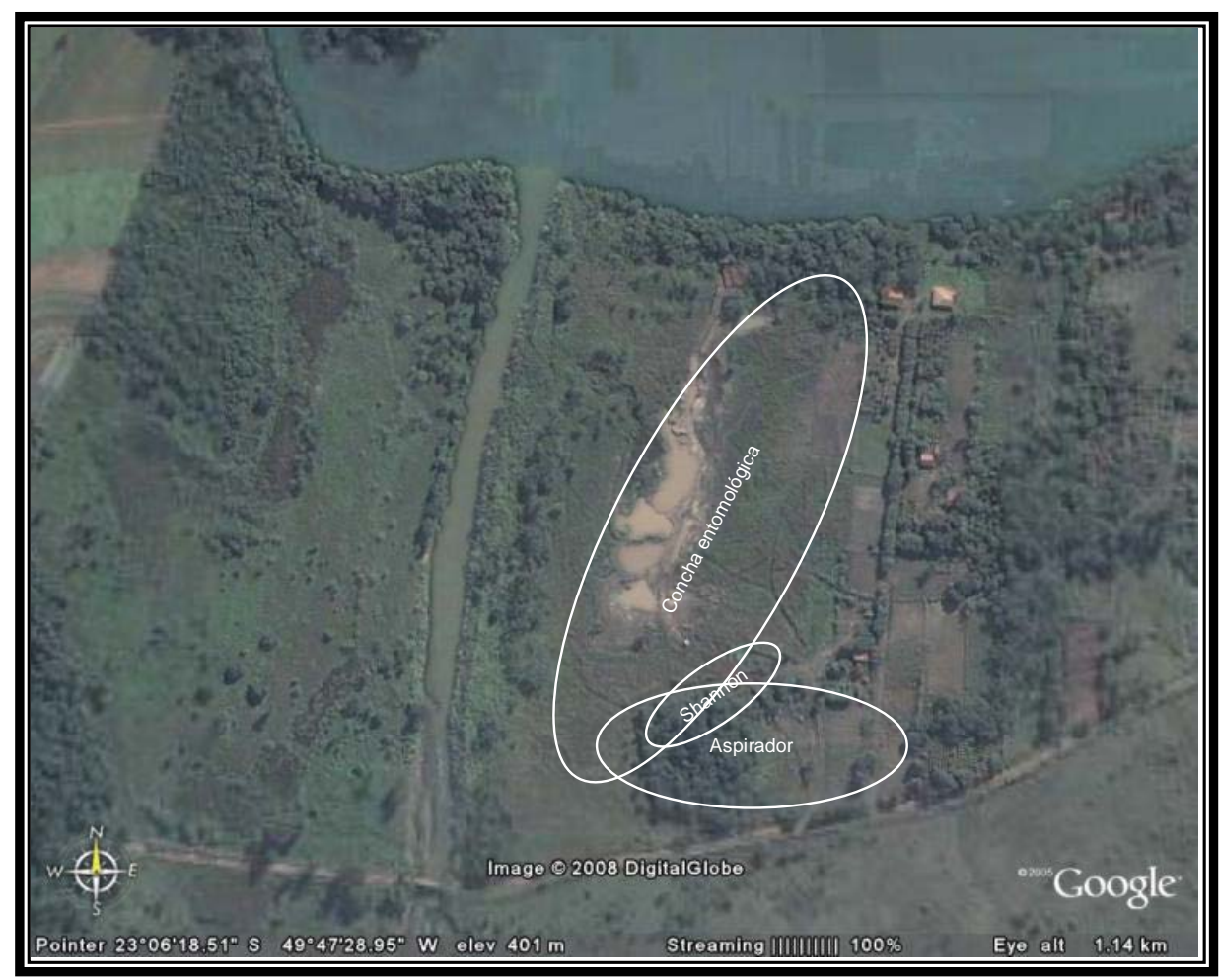

Figura 37. Estação de coleta de culicídeos Córrego Anhumas - localizada na margem esquerda do Rio Paranapanema, no município de Ribeirão Claro, PR. São mostradas as áreas onde foram feitas as coletas com armadilha de Shannon, com aspirador elétrico e concha entomológica. 
Anexo II - Paisagens das estações de coleta de mosquitos

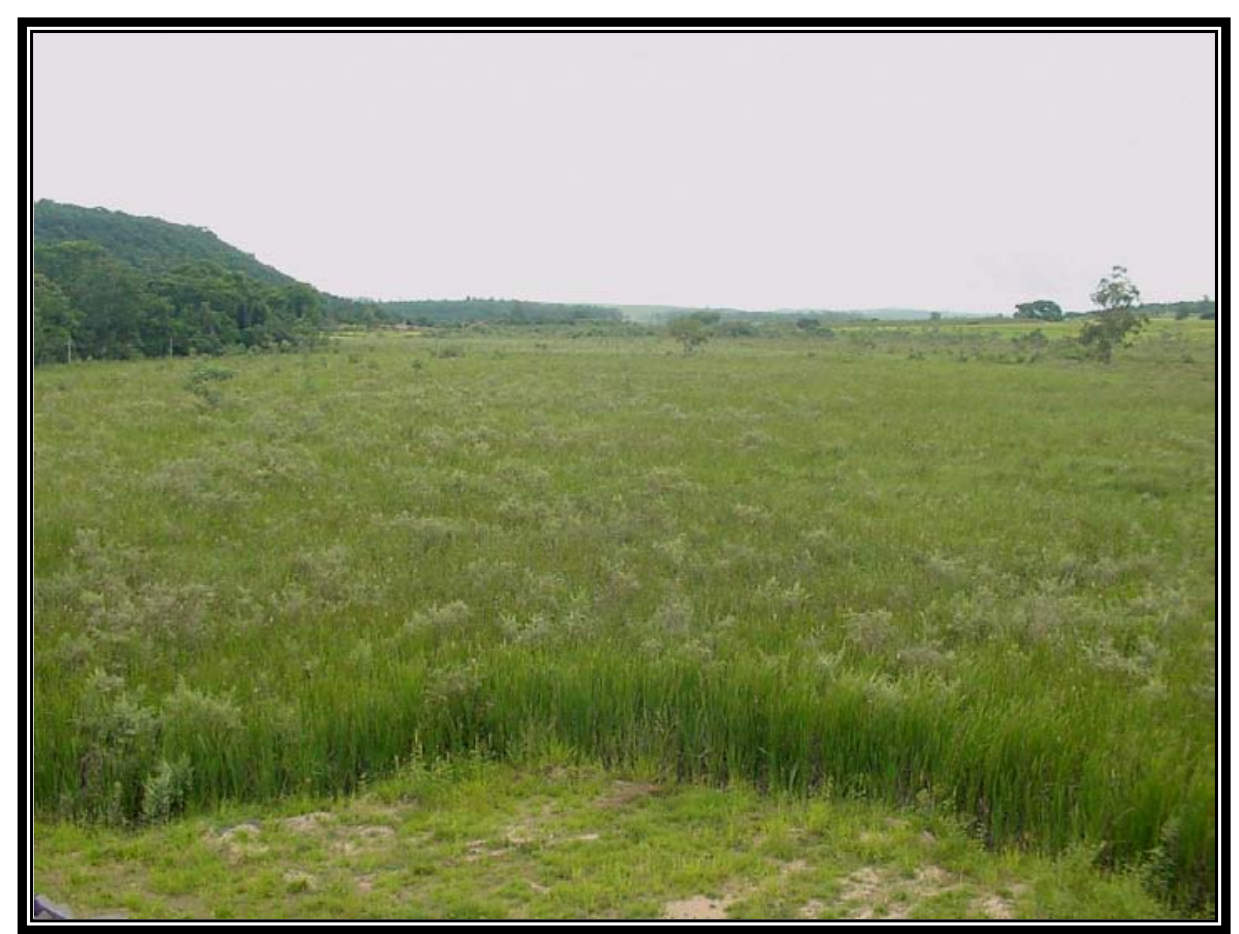

Figura 38. Estação de coleta de culicídeos Canteiro de Obras - vista a partir das instalações do canteiro. Em primeiro plano observa-se o domínio da taboa (Typha sp.). Ao fundo, à esquerda destaca-se a mata onde foi instalada a armadilha de Shannon em sua borda.

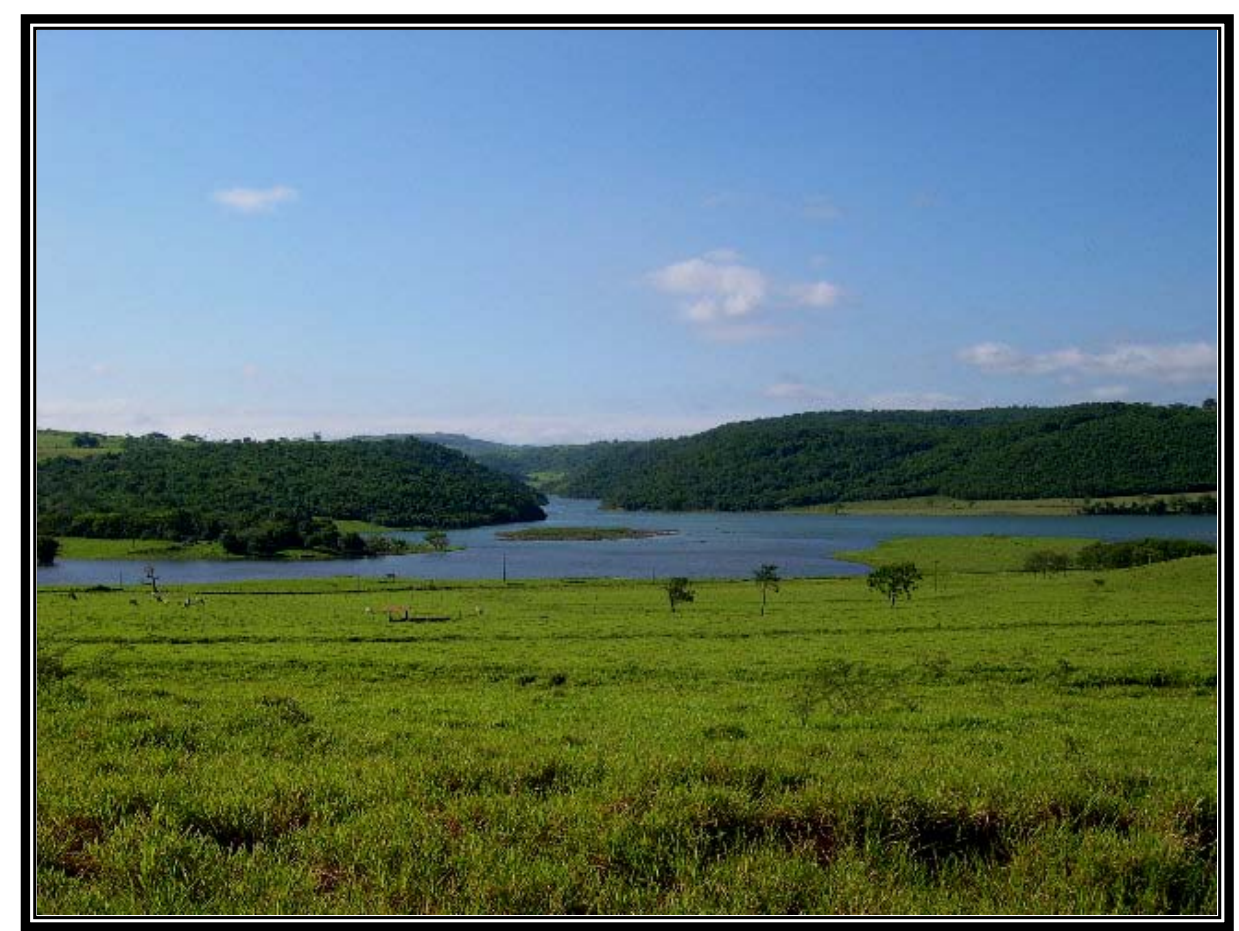

Figura 39. Aspecto do lago recém-formado na região da estação de coleta Sítio Caiçara. Em primeiro plano observa-se área de pastagem, no segundo a área inundada.. No canto esquerdo superior observa-se, em ponto mais elevado, a sede do sítio utilizado para coleta 


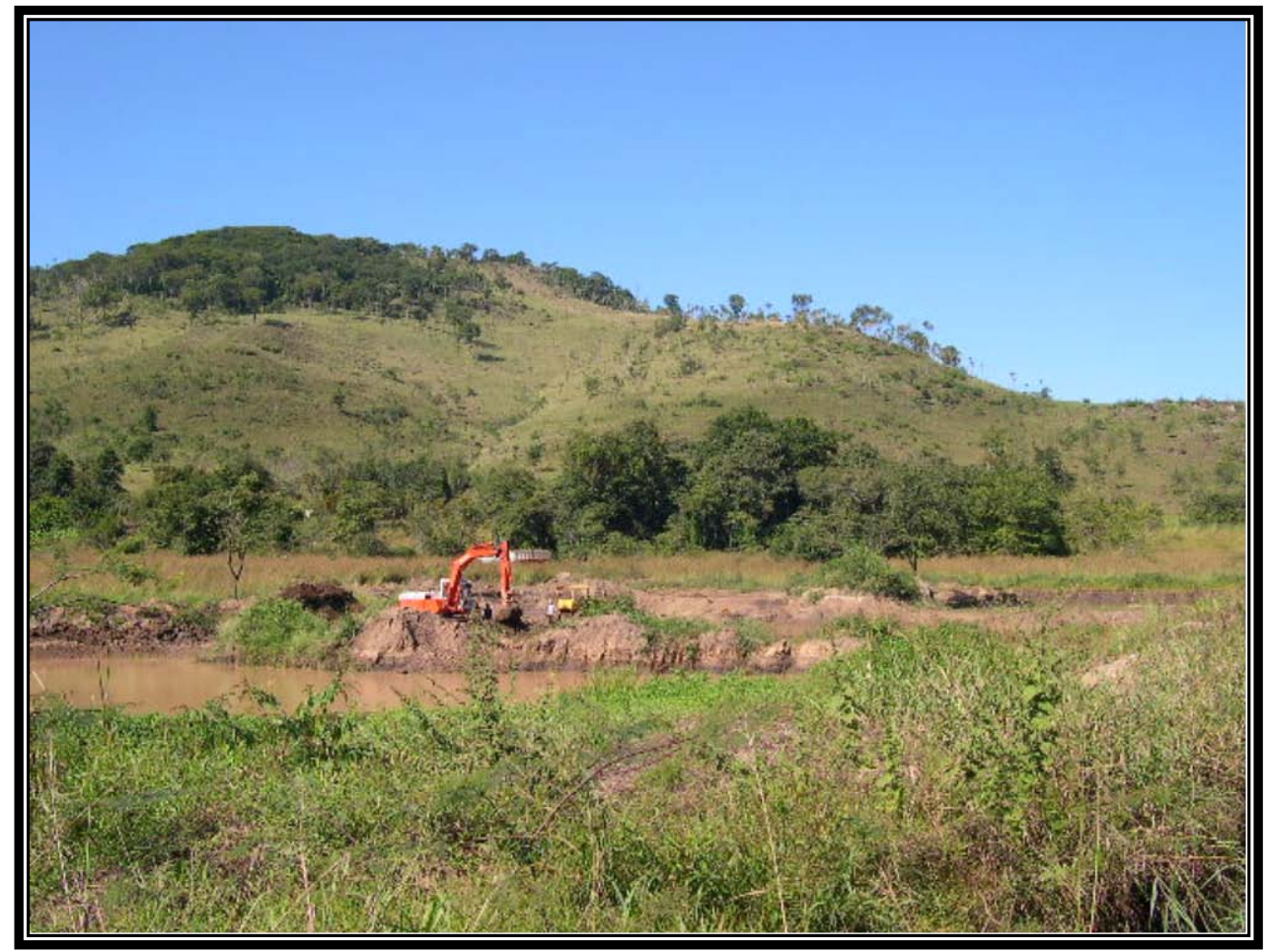

Figura 40. Estação de coleta de culicídeos Córrego Anhumas - vista a partir da margem esquerda do rio Paranapanema. Em primeiro plano observa-se área de extração de argila. Em segundo plano é mostrada a mata residual utilizada nas aspirações e na coleta com armadilha de Shannon. Ao fundo observa-se terreno montanhoso ocupado por pastagem. As coletas de formas imaturas foram feitas no brejo visto no primeiro plano. 


\section{Anexo III - Técnicas de coleta de mosquitos}

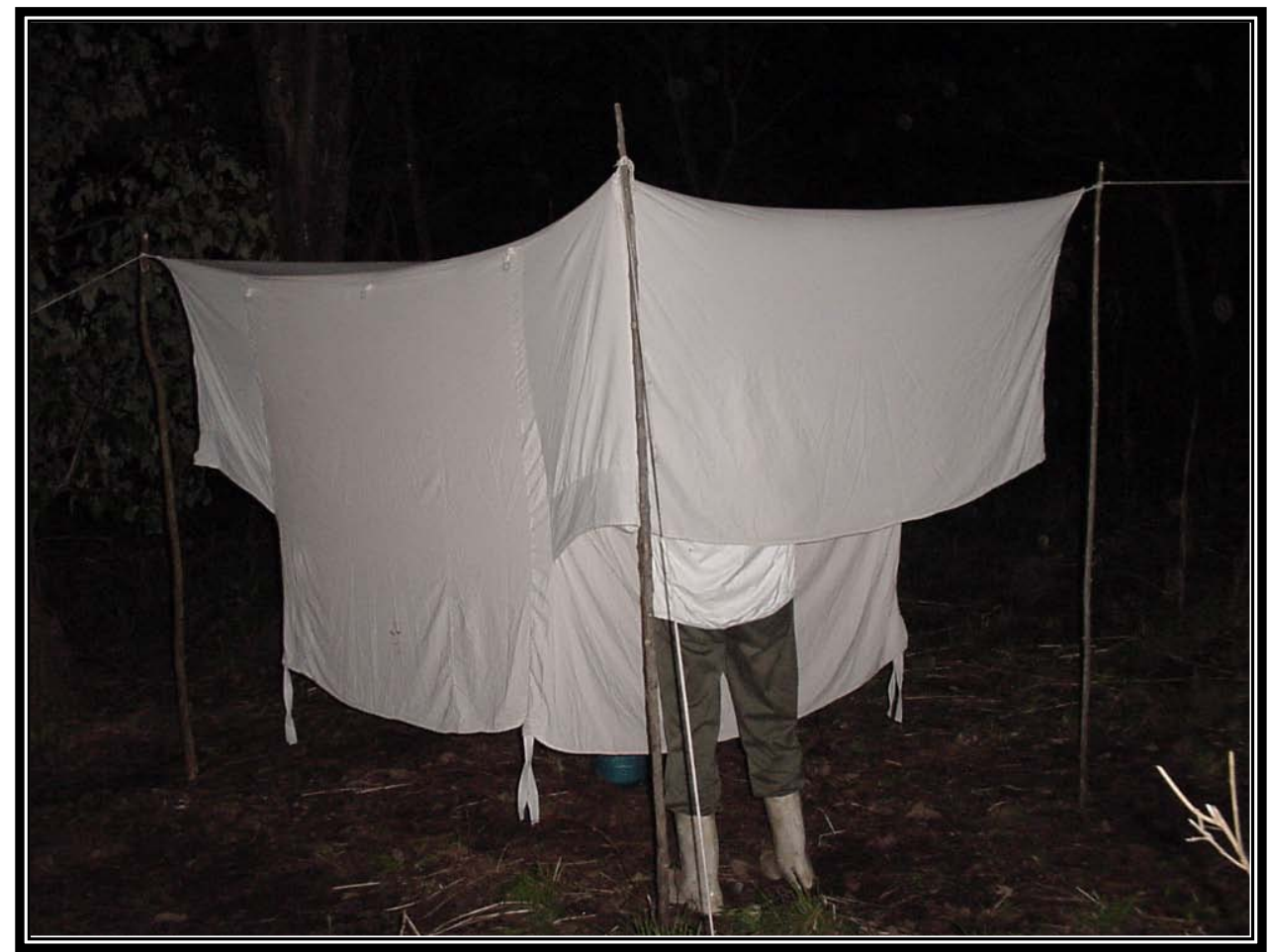

Figura 41. Armadilha de Shannon instalada na borda da mata residual localizada na estação de coleta do Canteiro de Obras, no período pré-inundação

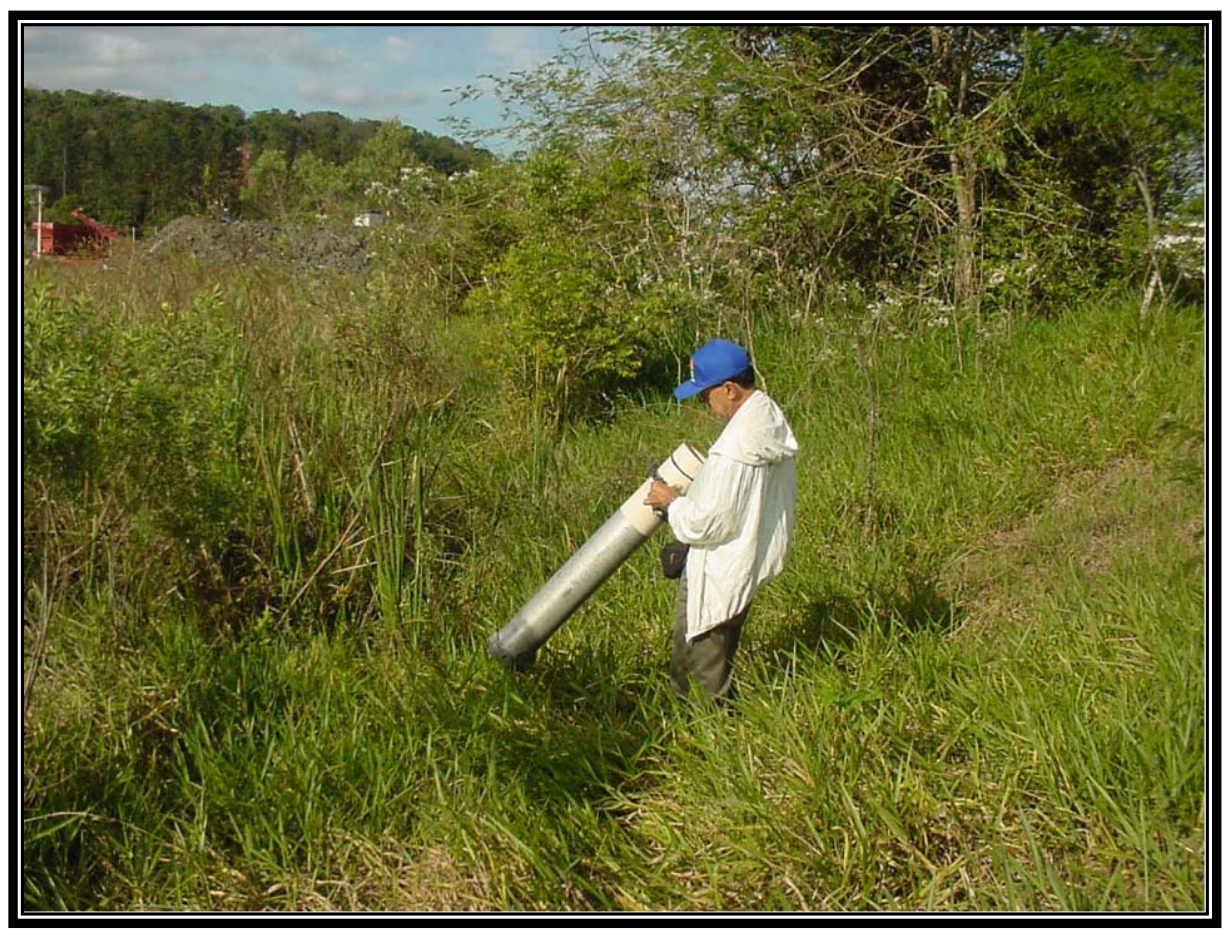

Figura 42. Aspirador elétrico em operação na margem do brejo da estação de coleta do Canteiro de Obras, no período pré-inundação. 


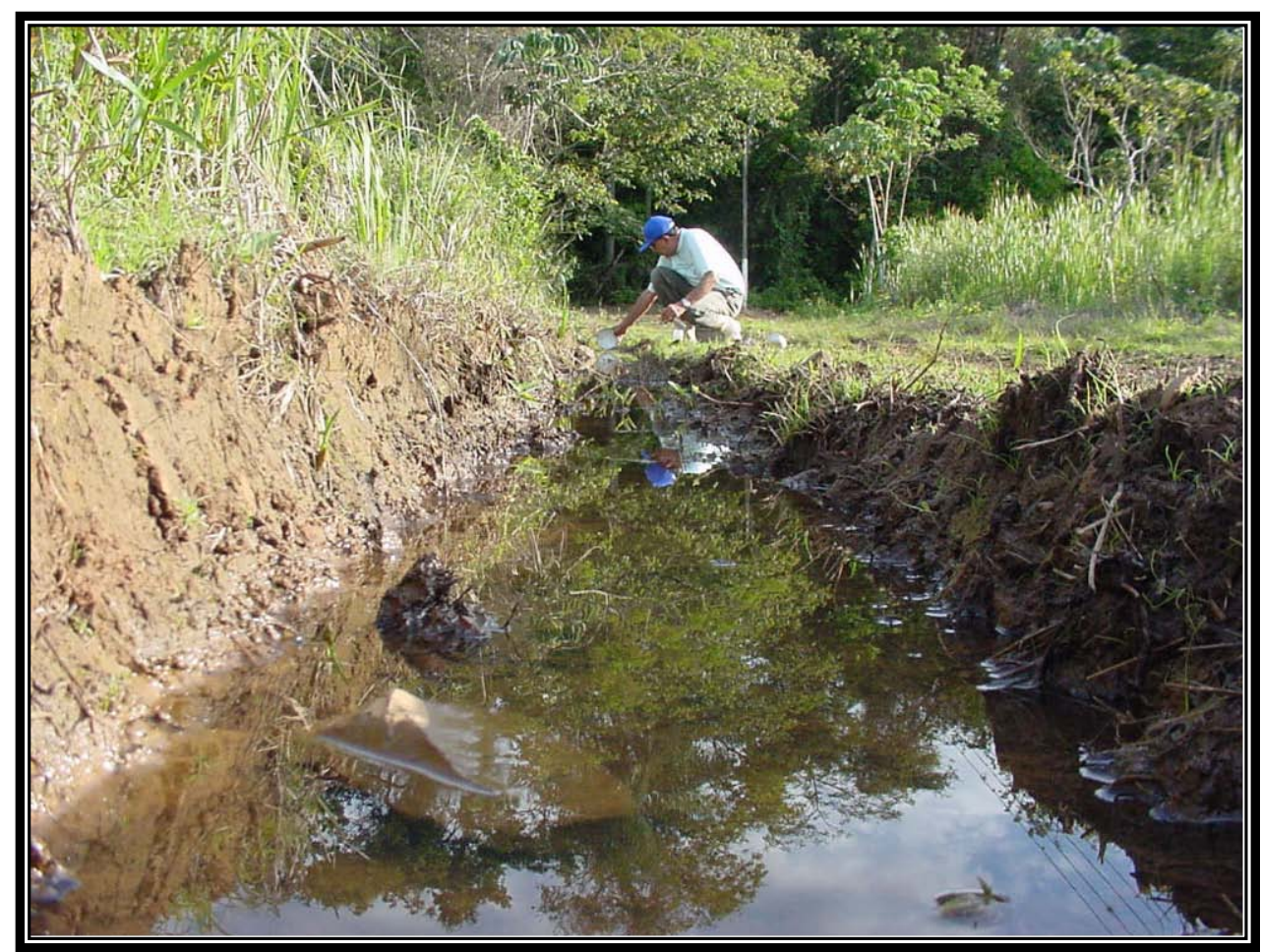

Figura 43. Coleta de mosquitos imaturos com concha entomológica em vala na margem do caminho na estação do Canteiro de Obras, no período pré-inundação.

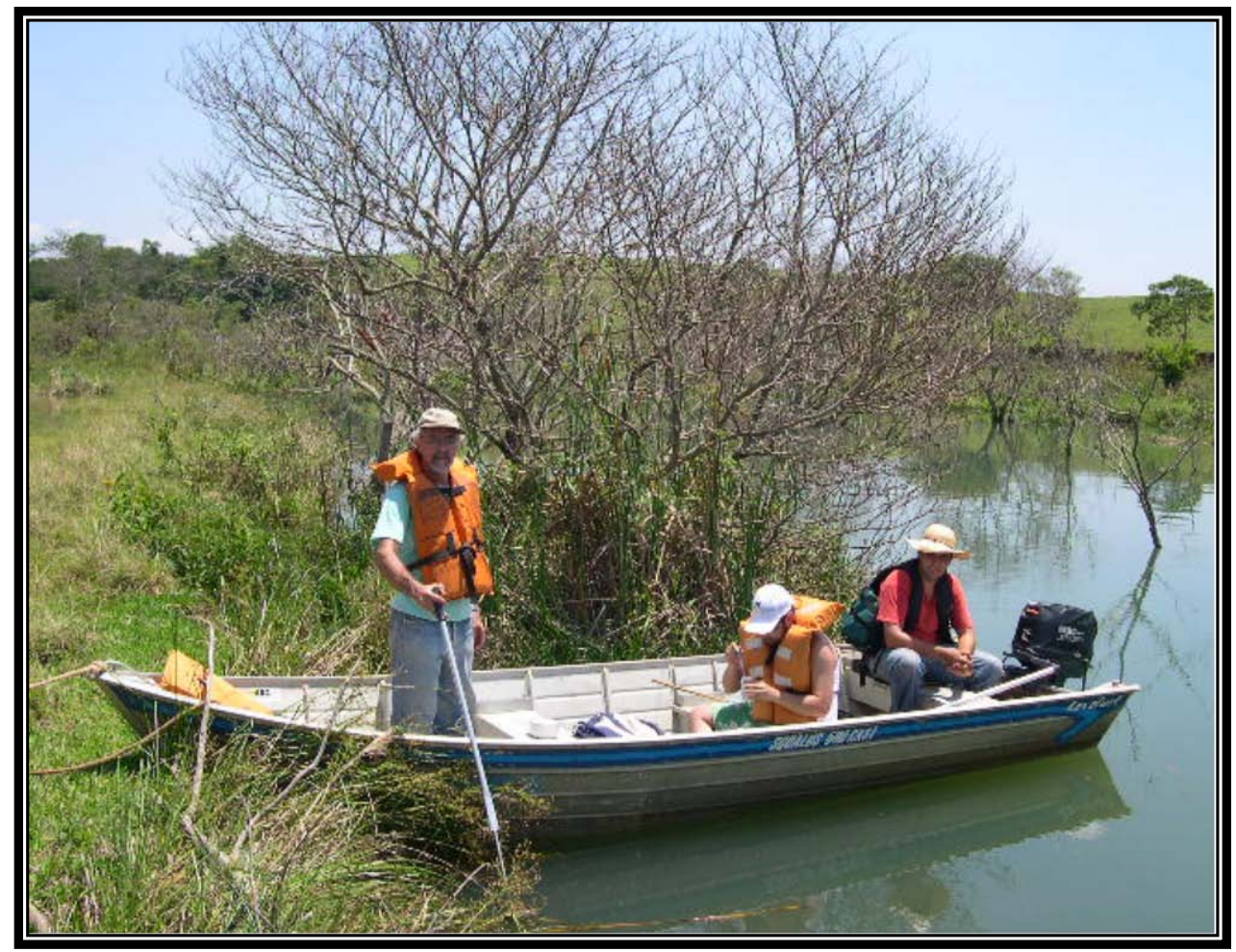

Figura 44. Equipe em operação de coleta de mosquitos imaturos, mediante o uso de um barco, na margem direita do lago recém-formado, em posição oposta à estação Córrego Anhumas. 


\section{Anexo VI-Banco de dados brutos}

Tabela 16. Número de mosquitos segundo as espécies coletados com concha entomológica na fase imatura, no Canteiro de Obras, durante o monitoramento da hidrelétrica de Ourinhos, no período de setembro de 2002 a outubro de 2007.

\begin{tabular}{|c|c|c|c|c|c|c|c|c|c|c|c|c|c|}
\hline \multirow{2}{*}{ Espécie } & \multicolumn{7}{|c|}{ Pré } & \multicolumn{6}{|c|}{ Pós } \\
\hline & $23 / 9 / 2002$ & $21 / 1 / 2003$ & $5 / 8 / 2003$ & 1/1/2/2003 & $29 / 5 / 2004$ & $1 / 12 / 2004$ & Total & 13/11/2005 & $3 / 6 / 2006$ & $17 / 11 / 2006$ & $18 / 5 / 2007$ & $28 / 10 / 2007$ & Total \\
\hline Ae. albopictus & & 2 & & & & & \begin{tabular}{|l|l|}
2 \\
\end{tabular} & 2 & & & & & 2 \\
\hline Ae. fluviatilis & & & & & 8 & 14 & 22 & 75 & & & & & 75 \\
\hline \begin{tabular}{|l|} 
Ae. scapularis \\
\end{tabular} & 3 & & & 1 & 86 & & 90 & & & & & 3 & 3 \\
\hline An. (Nys.) albitarsis & & & 4 & & & & 4 & & 3 & & 2 & & 5 \\
\hline An. (Nys.) galvaoi & & & 3 & & & & 3 & & & & & & \\
\hline An. (Nys.) sp & & 5 & & & & & 5 & & & & 1 & & 1 \\
\hline An. (Nys.) strodei/ rondoni & & 3 & 1 & & & & 4 & & & & & & \\
\hline \begin{tabular}{|l|} 
An. argyritarsis \\
\end{tabular} & 13 & 6 & & & 1 & & 20 & & 4 & & 2 & & 6 \\
\hline Cx. (Cux.) chidesteri & & & 1 & & & & 1 & & & & & & \\
\hline Cx. (Cux.) coronator & 3 & 5 & & & 11 & & 19 & & 22 & 3 & 7 & 4 & 36 \\
\hline CX. (Cux.) declarator & & & & 2 & & & 2 & & 1 & & 1 & & 2 \\
\hline CX. (Cux.) dolosus & & & & & & & & & & & 1 & 1 & 2 \\
\hline CX. (Cux.) grupo Coronator & 30 & 28 & & & 10 & & 68 & & 60 & 36 & 16 & 9 & 121 \\
\hline CX. (Cux.) mollis & & & & 2 & & & 2 & & & & & & \\
\hline Cx. (Cux.) quinquefascistus & & & & & & 10 & 10 & 38 & & & & & 38 \\
\hline Cx. (Cux.) sp. & 1 & 11 & & 10 & & & 22 & & & 4 & 1 & & 5 \\
\hline CX. (Mcx.) imitator & & & & & & & & & & 10 & & & 10 \\
\hline \begin{tabular}{|l|} 
CX. (Mel.) ribeirensis \\
\end{tabular} & 1 & & 2 & & & & 3 & & & & & & \\
\hline $\begin{array}{l}\text { CX. (Mel.) seção Melanoconion } \\
\end{array}$ & & & & & & & & & 1 & & & & 1 \\
\hline Ps. ciliata & & & & & & 5 & 5 & & & & & & \\
\hline \begin{tabular}{|l} 
Ps. confinnis \\
\end{tabular} & & & & & 5 & & 5 & & & & & & \\
\hline Ps. ferox & 1 & & & & & & 1 & & & & & & \\
\hline Sa.sp. & & & & & & & & & & 1 & & & 1 \\
\hline Ur. (Ura.) lowii & & & & & & & & & 1 & 1 & & & 2 \\
\hline Ur. (Ura.) pulcherrima & & & & & & & & & & 1 & 1 & & 2 \\
\hline Ur. geometrica & & & 3 & & & & 3 & 9 & & 3 & & 11 & 23 \\
\hline \begin{tabular}{|l|l} 
Ur. nataliae \\
\end{tabular} & & & 2 & & & & 2 & & & & & & \\
\hline Wy. rooti & & & & & & & & & & 16 & & & 16 \\
\hline Wy.sp. & & & & 1 & & & 1 & & & & 9 & & 9 \\
\hline total & 52 & 60 & 16 & 16 & 121 & 29 & 294 & 124 & 92 & 75 & 41 & 28 & 360 \\
\hline
\end{tabular}

Tabela 17. Número de mosquitos segundo as espécies coletados com concha entomológica na fase imatura, no Sítio Caiçara, durante o monitoramento da hidrelétrica de Ourinhos, no período de setembro de 2002 a outubro de 2007.

\begin{tabular}{|c|c|c|c|c|c|c|c|c|c|c|c|c|c|}
\hline \multirow{2}{*}{ Espécie } & \multicolumn{7}{|c|}{ Pré } & \multicolumn{6}{|c|}{ Pós } \\
\hline & 23/9/2002 & $21 / 1 / 2003$ & 5/8/2003 & 1/1/2/2003 & $29 / 5 / 2004$ & \begin{tabular}{|l|}
$\mathbf{1} / \mathbf{1 2} / \mathbf{2 0 0 4}$ \\
\end{tabular} & \begin{tabular}{|c|} 
Total \\
\end{tabular} & \begin{tabular}{|l|}
$13 / 11 / 2005$ \\
\end{tabular} & $3 / 6 / 2006$ & 17/11/2006 & 18/5/2007 & \begin{tabular}{|l|}
$28 / 10 / 2007$ \\
\end{tabular} & \begin{tabular}{|l|} 
Total \\
\end{tabular} \\
\hline \begin{tabular}{|l} 
Ad. squamipennis \\
\end{tabular} & & & & & & & & & 1 & 4 & 62 & & \begin{tabular}{|l|l|}
67 \\
\end{tabular} \\
\hline \begin{tabular}{|l} 
Ae. albopictus \\
\end{tabular} & & 1 & & & 1 & 10 & 12 & & & & & & \\
\hline \begin{tabular}{|l|} 
Ae. fluviatilis \\
\end{tabular} & & & & & & & & & & & & 22 & 22 \\
\hline Ae. scapularis & 26 & & & 1 & 5 & & 32 & & & & & & \\
\hline \begin{tabular}{|l|} 
Ae. terrens \\
\end{tabular} & 15 & 2 & & & & & 17 & & & & & & \\
\hline An. (Nys.) albitarsis & & & & 2 & & & 2 & & 9 & 3 & 18 & 4 & 34 \\
\hline \begin{tabular}{|l} 
An. (Nys.) darlingi \\
\end{tabular} & & & & & & & & & & & 3 & & 3 \\
\hline \begin{tabular}{|l|} 
An. (Nys.) sp \\
\end{tabular} & & & & & & & & & & 1 & & 5 & 6 \\
\hline \begin{tabular}{|l|} 
An. (Nys.) strodei \\
\end{tabular} & & & & & & & & & 3 & & 4 & & 7 \\
\hline An. (Nys.) strodei/ rondoni & 2 & & 5 & & & & 7 & & & & & & \\
\hline \begin{tabular}{|l|} 
An. (Nys.) triannulatus \\
\end{tabular} & & & & & & & & & & & 11 & & 11 \\
\hline \begin{tabular}{|l|} 
An. argyritarsis \\
\end{tabular} & & 1 & & & & & 1 & & 2 & & 5 & 5 & 12 \\
\hline Cx. (Cux.) chidesteri & 3 & & & & & & 3 & & & 11 & & & 11 \\
\hline \begin{tabular}{|l} 
Cx. (Cux.) coronator \\
\end{tabular} & & 7 & 4 & 1 & 15 & 17 & 44 & & & 1 & 4 & 1 & 6 \\
\hline \begin{tabular}{|l} 
Cx. $($ Cux. $)$ declarator \\
\end{tabular} & & & 6 & & 1 & & 7 & & & & & & \\
\hline \begin{tabular}{|l|} 
Cx. (Cux.) dolosus \\
\end{tabular} & & & 30 & & 15 & & 45 & & & & & & \\
\hline \begin{tabular}{|l} 
Cx. (Cux.) dolosus/eduardoi \\
\end{tabular} & & & & & 5 & & 5 & & & & & & \\
\hline Cx. (Cux.) eduardoi & & & & & 2 & & 2 & & & & & & \\
\hline CX. (Cux.) grupo Coronator & & 5 & & 21 & 25 & 34 & 85 & & 3 & & 12 & 1 & 16 \\
\hline \begin{tabular}{|l|} 
CX. (Cux.) mollis \\
\end{tabular} & & & 4 & 2 & & & 6 & & 1 & 5 & & & 6 \\
\hline \begin{tabular}{|l|} 
Cx. (Cux.) sp. \\
\end{tabular} & 6 & 2 & & 13 & & & 21 & & & & 1 & 2 & 3 \\
\hline CX. (Mel.) aureonotatus & & & & & & & & & & 3 & & & 3 \\
\hline CX. (Mel.) dureti & & & & & & & & & & & 1 & & 1 \\
\hline \begin{tabular}{|l} 
Cx. (Mel.) seção Melanoconion \\
\end{tabular} & & & & & & & & & & & 2 & & 2 \\
\hline \begin{tabular}{|l|}
$C X .(M e l)$. \\
Sp
\end{tabular} & & & & & & & & & 4 & & & & 4 \\
\hline Cx. (Mel.) vaxus & & & & & & & & & 1 & & 1 & & 2 \\
\hline Ur. (Ura.) lowii & & & & & & & & & & 3 & & & 3 \\
\hline Ur. (Ura.) sp. & & & & & & & & & & & 1 & & 1 \\
\hline Total & 52 & 18 & 49 & 40 & 69 & 61 & 289 & & 24 & 31 & 125 & 40 & 220 \\
\hline
\end{tabular}


Tabela 18. Número de mosquitos segundo as espécies coletados com concha entomológica na fase imatura, no Córrego Anhumas, durante o monitoramento da hidrelétrica de Ourinhos, no período de setembro de 2002 a outubro de 2007.

\begin{tabular}{|c|c|c|c|c|c|c|c|c|c|c|c|c|c|}
\hline \multirow{2}{*}{ Espécie } & \multicolumn{7}{|c|}{ Pré } & \multicolumn{6}{|c|}{ Pós } \\
\hline & $23 / 9 / 2002$ & $21 / 1 / 2003$ & $5 / 8 / 2003$ & $1 / 12 / 2003$ & 29/5/2004 & 1/1/2/2004 & Total & 13/11/2005 & 3/6/2006 & 17/11/2006 & $18 / 5 / 2007$ & $28 / 10 / 2007$ & Total \\
\hline \begin{tabular}{|l} 
Ad. squamipennis \\
\end{tabular} & 12 & 3 & 23 & & & & 38 & 32 & 26 & 11 & 15 & 9 & 93 \\
\hline \begin{tabular}{|l|} 
Ae. scapularis \\
\end{tabular} & 1 & & & 13 & 36 & & 50 & & & & & & \\
\hline Ae. serratus & & & & & 1 & & 1 & & & & & & \\
\hline An. (Nys.) albitarsis & & & 1 & & 1 & 8 & 10 & 1 & 16 & 14 & 7 & 26 & 64 \\
\hline \begin{tabular}{|l|} 
An. (Nys.) galvaoi \\
\end{tabular} & & & & & 1 & & 1 & & & & & & \\
\hline \begin{tabular}{|l|}
$A n$. (Nys.) sp \\
\end{tabular} & & 2 & & 1 & & & 3 & & & & & 2 & 2 \\
\hline An. (Nys.) strodei & & & & & & & & & 1 & & & & 1 \\
\hline \begin{tabular}{|l|} 
An. argyritarsis \\
\end{tabular} & 9 & 19 & & & 15 & & 43 & & 1 & & & 1 & 2 \\
\hline \begin{tabular}{|l|} 
CX. (Cux.) bidens \\
\end{tabular} & & & & & & & & & & & & 1 & 1 \\
\hline \begin{tabular}{|l} 
CX. (Cux.) chidesteri \\
\end{tabular} & & & 7 & & & & 7 & & & & & & \\
\hline \begin{tabular}{|l|} 
Cx. (Cux.) coronator \\
\end{tabular} & 5 & & & 7 & 4 & & 16 & & 2 & & & 1 & 3 \\
\hline \begin{tabular}{|l} 
CX. $($ Cux. $)$ dolosus \\
\end{tabular} & & & & & 1 & & 1 & & & & & & \\
\hline \begin{tabular}{|l|} 
Cx. (Cux.) grupo Coronator \\
\end{tabular} & 5 & & & 6 & 12 & & 23 & & 28 & & & 9 & 37 \\
\hline \begin{tabular}{|l} 
Cx. (Cux.) quinquefascistus \\
\end{tabular} & & & & & & & & & & & 1 & & 1 \\
\hline $\begin{array}{l}\text { Cx. (Cux.) sp. } \\
\end{array}$ & & & & 3 & & & 3 & & 1 & & & 9 & 10 \\
\hline \begin{tabular}{|l|}
$C X .(M C X$.$) imitator$ \\
\end{tabular} & & & & 5 & & & 5 & & & & & & \\
\hline CX. (Mel.) aureonotatus & & & & & & & & & & 3 & & & 3 \\
\hline Cx. (Mel.) bastagarius & & & & & & 1 & 1 & & & & & & \\
\hline \begin{tabular}{|l} 
Cx. (Mel.) seção Melanoconion \\
\end{tabular} & & 1 & & & & & 1 & & 3 & 1 & & & 4 \\
\hline \begin{tabular}{|l|} 
Cx. (Mel.) sp. \\
\end{tabular} & & 2 & & & & & 2 & & & & & & \\
\hline Cx. (Mel.) vaxus & & & & & & 2 & 2 & & & & & 2 & 2 \\
\hline Ps. cingulata & & & & 2 & & & 2 & & & & & & \\
\hline \begin{tabular}{|l|} 
PS. confinnis \\
\end{tabular} & & & & & 1 & & 1 & & & & & & \\
\hline \begin{tabular}{|l|} 
Ps. sp. \\
\end{tabular} & 2 & & & & & & 2 & & & & & & \\
\hline \begin{tabular}{|l} 
Ur. (Ura.) lowii \\
\end{tabular} & & & & & 1 & 1 & 2 & & & & & & \\
\hline \begin{tabular}{|l|} 
Ur. geometrica \\
\end{tabular} & & & 1 & & & & 1 & & & & & & \\
\hline Ur. (Ura.) pulcherrima & 1 & & 2 & & & & 3 & 2 & & & & & 2 \\
\hline Ur. sp & & & & & & & & & & & & 1 & 1 \\
\hline Wy. medioalbipes & & & & 24 & & & 24 & & & & & & \\
\hline Total & 35 & 27 & 34 & 61 & 73 & 12 & 242 & 35 & 78 & 29 & 23 & 61 & 226 \\
\hline
\end{tabular}

Tabela 19. Número de mosquitos segundo as espécies coletados com aspirador no Canteiro de Obras, durante o monitoramento da hidrelétrica de Ourinhos, no período de setembro de 2002 a outubro de 2007.

\begin{tabular}{|c|c|c|c|c|c|c|c|c|c|c|c|c|c|}
\hline \multirow{2}{*}{ Espécie } & \multicolumn{7}{|c|}{ Pré } & \multicolumn{6}{|c|}{ Pós } \\
\hline & $23 / 9 / 2002$ & $21 / 1 / 2003$ & $5 / 8 / 2003$ & $1 / 12 / 2003$ & $29 / 5 / 2004$ & 1/12/2004 & Total & 13/11/2005 & $3 / 6 / 2006$ & 17/11/2006 & $18 / 5 / 2007$ & $28 / 10 / 2007$ & Total \\
\hline Ae. albopictus & & & & & & & & 1 & & & & & 1 \\
\hline Ae. scapularis & & & & & 9 & & 9 & & & 2 & & & 2 \\
\hline Cq. albicosta & & 14 & & & & & 14 & & & & & & \\
\hline Cq. chrysonotum & & & & & & 2 & 2 & & & 2 & & & 2 \\
\hline Cq. hermanoi & & & & & & 1 & 1 & & & & & & \\
\hline Cq. venezuelensis & & & & & 1 & & 1 & 5 & & & & & 5 \\
\hline CX. (Ads.) amazonenzis & & & & & 1 & & 1 & & & & & & \\
\hline CX. (Cux.) coronator & & & & & 1 & & 1 & & & & & & \\
\hline CX. (Cux.) declarator & 1 & & & 2 & & & 3 & 1 & & & & & 1 \\
\hline \begin{tabular}{|l|l} 
Cx. (Cux.) dolosus \\
\end{tabular} & & & & 1 & 1 & & 2 & 1 & & 3 & & 2 & 6 \\
\hline CX. (Cux.) dolosus/eduardoi & & & & & & 2 & 2 & & & & & & \\
\hline CX. (Cux.) grupo Coronator & & & & & 1 & & 1 & & & & & & \\
\hline \begin{tabular}{|l|} 
CX. (Cux.) nigripalpus \\
\end{tabular} & 1 & & & & & & 1 & & & & & & \\
\hline Cx. (Cux.) quinquefascistus & & & & & & & & 1 & & & & & 1 \\
\hline Cx. (Cux.) sp. & 1 & 1 & & 2 & & 2 & 6 & & & 3 & 2 & 3 & 8 \\
\hline $\begin{array}{l}\text { Cx. (Mel.) grupo Atratus } \\
\end{array}$ & & & & & & & & & & & 1 & & 1 \\
\hline CX. (Mel.) ocoss & & & & & 1 & & 1 & & & & & & \\
\hline CX. (Mel.) plectoporpe & & 1 & & & & & 1 & & & & & & \\
\hline Cx. (Mel.) rabelloi & & & & & & & & 1 & & & & & 1 \\
\hline CX. (Mel.) ribeirensis & 1 & & & & 1 & & 2 & & & & & & \\
\hline \begin{tabular}{|l} 
Cx. (Mel.) seção Melanoconion \\
\end{tabular} & & 2 & & & 1 & & 3 & & & & & & \\
\hline CX. (Mel.) sphatulatus & & & & & & & & 1 & & & & & 1 \\
\hline Cx. bidens & & & & & & & & & & 1 & & & 1 \\
\hline \begin{tabular}{|l|} 
Ps. albigenu \\
\end{tabular} & & & & 1 & 1 & & 2 & & & & & & \\
\hline Ps. ferox & & & & & & & & & & 1 & & & 1 \\
\hline Ps. lutzii & & & & & & 1 & 1 & & & & & & \\
\hline Ur. geometrica & & & & 2 & & & 2 & & & & & & \\
\hline Ur. mathesoni & & 1 & 1 & & 1 & 2 & 5 & & & & 3 & 1 & 4 \\
\hline Ur. nataliae & & & & 1 & & & 1 & & & & & $\frac{1}{1}$ & 1 \\
\hline Ur. (Ura.) pulcherrima & 1 & & & & & 2 & 3 & & & 1 & & & 1 \\
\hline Wy. sp. & 1 & 3 & & & & & 4 & & & & & & \\
\hline Total & 6 & 22 & 1 & 9 & 19 & 12 & 69 & 11 & & 13 & 6 & 7 & 37 \\
\hline
\end{tabular}


Tabela 20. Número de mosquitos segundo as espécies coletados com aspirador no Sítio Caiçara, durante o monitoramento da hidrelétrica de Ourinhos, no período de setembro de 2002 a outubro de 2007.

\begin{tabular}{|c|c|c|c|c|c|c|c|c|c|c|c|c|c|}
\hline \multirow{2}{*}{ Espécie } & \multicolumn{7}{|c|}{ Pré } & \multicolumn{6}{|c|}{ Pós } \\
\hline & $23 / 9 / 2002$ & $21 / 1 / 2003$ & $5 / 8 / 2003$ & 1/12/2003 & \begin{tabular}{|l|}
$29 / 5 / 2004$ \\
\end{tabular} & $1 / 12 / 2004$ & Total & 13/11/2005 & 3/6/2006 & $17 / 11 / 2006$ & $18 / 5 / 2007$ & \begin{tabular}{|l|}
$28 / 10 / 2007$ \\
\end{tabular} & Total \\
\hline \begin{tabular}{|l} 
Ad. squamipennis \\
\end{tabular} & & & & & & & & & & & & \begin{tabular}{|l|}
1 \\
\end{tabular} & 1 \\
\hline Ae. albopictus & & 1 & 3 & 3 & & & 7 & & & & & & \\
\hline \begin{tabular}{|l} 
Ae. crinifer \\
\end{tabular} & & & & & & & & 8 & & & & & 8 \\
\hline Ae. nubilus & & & & & & & & 1 & & & & & 1 \\
\hline Ae. scapularis & 2 & 8 & & 2 & 5 & 1 & 18 & 7 & & & & & 7 \\
\hline \begin{tabular}{|l|} 
Ae. serratus \\
\end{tabular} & & & & & & & & 1 & & & & & 1 \\
\hline Ae. serratus/nubilus & & & & & & & & 3 & & & & & 3 \\
\hline An. (Nys.) darlingi & & & & & & & & & & & 1 & & 1 \\
\hline \begin{tabular}{|l|} 
Cq. juxtamansonia \\
\end{tabular} & & & & & & & & 1 & & & & & 1 \\
\hline \begin{tabular}{|l|} 
Cq. venezuelensis \\
\end{tabular} & & 2 & & & & & 2 & 1 & 1 & & 2 & 2 & 6 \\
\hline Cx. (Cux.) chidesteri & & & & 1 & & & 1 & & & & & 4 & 4 \\
\hline CX. (Cux.) coronator & & 2 & & & 1 & & 3 & 3 & & & & & 3 \\
\hline CX. (Cux.) declarator & 2 & 9 & & 1 & 6 & 1 & 19 & & & & & & \\
\hline CX. (Cux.) dolosus & 2 & 3 & 1 & 1 & 19 & & 26 & 5 & & & & & 5 \\
\hline \begin{tabular}{|l|l|} 
Cx. (Cux.) dolosus/eduardoi \\
\end{tabular} & & & & & & 2 & 2 & & 1 & & & & 1 \\
\hline CX. (Cux.) grupo Coronator & & 7 & & & 16 & & 23 & & & & & & \\
\hline CX. (Cux.) lygrus & & 1 & & 1 & 1 & & 3 & 1 & & & & & 1 \\
\hline \begin{tabular}{|l|} 
CX. (Cux.) mollis \\
\end{tabular} & & & 1 & & & & 1 & & & & & & \\
\hline CX. (Cux.) quinquefascistus & & & 2 & & & & 2 & & & & & & \\
\hline \begin{tabular}{|l|} 
Cx. (Cux.) sp. \\
\end{tabular} & 2 & & & & 6 & 2 & 10 & 10 & & 1 & & 7 & 18 \\
\hline \begin{tabular}{|l|} 
Cx. (Mel.) ocossa \\
\end{tabular} & & & & & 1 & & 1 & & & & & & \\
\hline \begin{tabular}{|l} 
CX. (Mel.) pilosus \\
\end{tabular} & 1 & & & & & & 1 & & & & & & \\
\hline Ur. (Ura.) pulcherrima & & 1 & & & & & 1 & & & & & & \\
\hline Wy. sp. & & & 1 & & & & 1 & 3 & & & & & 3 \\
\hline Total & 9 & 34 & 8 & 9 & 55 & 6 & 121 & 44 & 2 & 1 & 3 & 14 & 64 \\
\hline
\end{tabular}

Tabela 21. Número de mosquitos segundo as espécies coletados com aspirador no Córrego Anhumas, durante o monitoramento da hidrelétrica de Ourinhos, no período de setembro de 2002 a outubro de 2007.

\begin{tabular}{|c|c|c|c|c|c|c|c|c|c|c|c|c|c|}
\hline \multirow{2}{*}{ Espécie } & \multicolumn{7}{|c|}{ Pré } & \multicolumn{6}{|c|}{ Pós } \\
\hline & 23/9/2002 & $21 / 1 / 2003$ & 5/8/2003 & $1 / 12 / 2003$ & 29/5/2004 & $1 / 12 / 2004$ & Total & 13/11/2005 & 3/6/2006 & 17/11/2006 & 18/5/2007 & $28 / 10 / 2007$ & Total \\
\hline Ad. squamipennis & & & 1 & & & & \begin{tabular}{|l|l|}
1 & \\
\end{tabular} & & & & 2 & & 2 \\
\hline Ae. crinifer & & & $\frac{1}{1}$ & & & & 1 & 3 & & & & & 3 \\
\hline Ae. nubilus & & & & & 5 & & 5 & & & & & & \\
\hline Ae. scapularis & 1 & 7 & 2 & 7 & 4 & 1 & 22 & 2 & 1 & & & & 3 \\
\hline Ae. serratus & & 1 & & & 4 & & 5 & & & & & & \\
\hline An. albitarsis s.l & & 1 & & & & & 1 & & & 1 & & & 1 \\
\hline An. (Nys.) darlingi & & & & & & & & & & & 2 & & 2 \\
\hline An. evansae & & & & & & & & & & & 1 & & 1 \\
\hline An. galvaoi & & 2 & & & & & 2 & & & & 1 & & 1 \\
\hline An. strodei & & & & & & & & & & & 1 & & 1 \\
\hline An. triannulatus & & & & & & & & & & & 1 & & 1 \\
\hline Cq. juxtamansonia & & & & 1 & & & 1 & & & & & & \\
\hline Cq. venezuelensis & & 7 & & & & & 7 & & & & 1 & & 1 \\
\hline Cx. (Cux.) chidesteri & 2 & 3 & & & 4 & & 9 & & & 1 & & & 1 \\
\hline CX. (Cux.) coronator & 1 & 5 & & 1 & 1 & 1 & 9 & & & & & 1 & 1 \\
\hline CX. (Cux.) declarator & 2 & 7 & & 2 & 14 & 3 & 28 & 2 & & 1 & & 1 & 4 \\
\hline CX. (Cux.) dolosus & 1 & 11 & 1 & & $\frac{17}{2}$ & & 15 & & & & & $\frac{1}{1}$ & 1 \\
\hline CX. (Cux.) grupo Coronator & & 7 & & & 4 & & 11 & & 3 & 2 & & & 5 \\
\hline CX. (Cux.) lygrus & & & & & 1 & & 1 & & & & & & \\
\hline CX. (Cux.) mollis & & & & 1 & & & 1 & & & & & & \\
\hline CX. (Cux.) sp. & 4 & & & 1 & 1 & & 6 & 4 & 1 & 2 & 4 & 1 & 12 \\
\hline CX. (Mel.) grupo Atratus & & 1 & & & & & 1 & & & & & & \\
\hline Cx. (Mel.) ocossa & & & & & & & & & & & 1 & & 1 \\
\hline CX. (Mel.) ribeirensis & & 4 & & & 1 & & 5 & & & & & & \\
\hline CX. (Mel.) zeteki & & & & & & & & & & & 1 & & 1 \\
\hline CX. bidens & 1 & & & & & & 1 & & & 3 & & & 3 \\
\hline CX. saltanensis & & 1 & & & 1 & & $\frac{1}{2}$ & & & & & & \\
\hline \begin{tabular}{|l|} 
Li. durhami \\
\end{tabular} & & & & 1 & & & 1 & & & & & & \\
\hline Ma. flaveola & & & & & & & & & & & & 3 & 3 \\
\hline Ma. indubitans & & 1 & & & & & 1 & & & & & & \\
\hline Ma. titillans & & & & & & & & & & & & 1 & 1 \\
\hline Ma. wilsoni & & & & & & & & & & & & 1 & 1 \\
\hline $\begin{array}{l}\text { Ps. ciliata } \\
\end{array}$ & & & 1 & & & & 1 & & & & & & \\
\hline \begin{tabular}{|l|} 
Ps. ferox \\
\end{tabular} & & & & & 1 & & 1 & 1 & & & & & 1 \\
\hline Ur. geometrica & & 1 & & & & & 1 & & & & & & \\
\hline Ur. (Ura.) pulcherrima & & & & & & & & 4 & & & & & 4 \\
\hline Total & 12 & 59 & 6 & 14 & 43 & 5 & 139 & 16 & 5 & 10 & 15 & 9 & 55 \\
\hline
\end{tabular}


Tabela 22. Número de mosquitos segundo as espécies coletados com armadilha de Shannon no Canteiro de Obras, durante o monitoramento da hidrelétrica de Ourinhos, no período de setembro de 2002 a outubro de 2007.

\begin{tabular}{|c|c|c|c|c|c|c|c|c|c|c|c|c|c|}
\hline \multirow{2}{*}{ Espécie } & \multicolumn{7}{|c|}{ Pré } & \multicolumn{6}{|c|}{ Pós } \\
\hline & $23 / 9 / 2002$ & $21 / 1 / 2003$ & $5 / 8 / 2003$ & $1 / 12 / 2003$ & \begin{tabular}{l|l|}
$29 / 5 / 2004$ \\
\end{tabular} & $1 / 12 / 2004$ & \begin{tabular}{|l|} 
Total \\
\end{tabular} & $13 / 11 / 2005$ & $3 / 6 / 2006$ & $17 / 11 / 2006$ & \begin{tabular}{|l|}
$18 / 5 / 2007$ \\
\end{tabular} & $\begin{array}{l}28 / 10 / 2007 \\
\end{array}$ & Total \\
\hline \begin{tabular}{|l|} 
Ad. squamippenis \\
\end{tabular} & 6 & 4 & & 10 & 4 & 2 & \begin{tabular}{|c|}
26 \\
\end{tabular} & 4 & 3 & & & $\frac{2}{2}$ & 9 \\
\hline \begin{tabular}{|l} 
Ae. crinifer \\
\end{tabular} & & & & 1 & & & 1 & & & & & & \\
\hline Ae. fluviatilis & & & & 1 & & & 1 & & & & & & \\
\hline Ae. scapularis & 18 & 2 & & 5 & 41 & & 66 & 23 & & 1 & 2 & & 26 \\
\hline \begin{tabular}{|l|} 
Ae. serratus \\
\end{tabular} & & & & & 1 & 1 & 2 & 1 & & & & & 1 \\
\hline An. (Ano.) maculipes & & & & & & & & 1 & & & & & 1 \\
\hline An. albitarsis s.l & 6 & 1 & 8 & & & & 15 & & & & & & \\
\hline An. argyritarsis & & & 1 & & & & 1 & & & & & & \\
\hline An. evansae & & & & & 1 & 1 & 2 & & & & & & \\
\hline \begin{tabular}{|l|} 
An. galvaoi \\
\end{tabular} & 26 & 10 & & . & 3 & 2 & 41 & & 1 & & & & 1 \\
\hline An. lutzi & & & & & & & & 1 & & & & & $\frac{1}{1}$ \\
\hline An. strodei & & & & & & & & 2 & & & & & 2 \\
\hline An. triannulatus & 28 & 1 & & 6 & 14 & & 49 & & & & & & \\
\hline Cq. albicosta & & 598 & & 8 & 3 & 4 & 613 & 64 & & & & & 64 \\
\hline \begin{tabular}{|l|} 
Cq. chrysonotum \\
\end{tabular} & 39 & 24 & & & & 55 & 118 & 2 & & 4 & & 5 & 11 \\
\hline $\begin{array}{l}\text { Cq. chrysonotum/albifera } \\
\end{array}$ & 52 & 69 & & 42 & 6 & 628 & 797 & 611 & & 30 & 6 & 6 & 653 \\
\hline Cq. hermanoi & 1 & 1 & & 35 & 8 & 5 & 50 & 148 & & & 10 & 5 & 163 \\
\hline \begin{tabular}{|l|} 
Cq. juxtamansonia \\
\end{tabular} & 55 & 3 & 1 & 11 & 2 & 32 & 104 & 40 & & 3 & 1 & 2 & 46 \\
\hline \begin{tabular}{|l|} 
Cq. nigricans \\
\end{tabular} & & 2 & & 52 & & & 54 & 63 & & & & 3 & 66 \\
\hline \begin{tabular}{|l|} 
Cq. shannoni \\
\end{tabular} & 2 & 4 & & & 2 & 2 & 10 & & & & & & \\
\hline Cq. venezuelensis & 71 & 21 & & 11 & 11 & 9 & 123 & 354 & 1 & & 1 & & 356 \\
\hline Cx. (Ads.) amazonenzis & & 17 & & 3 & 3 & & 23 & 7 & & & & & 7 \\
\hline \begin{tabular}{|l|} 
Cx. (Cux.) dolosus \\
\end{tabular} & & & 1 & & & & 1 & 2 & & & & & 2 \\
\hline CX. (Cux.) dolosus/eduardoi & & & & & & & & & 4 & & & & 4 \\
\hline \begin{tabular}{|l} 
Cx. (Cux.) grupo Coronator \\
\end{tabular} & 1 & & & & 13 & & 14 & & 2 & & & & 2 \\
\hline \begin{tabular}{|l|} 
CX. (Cux.) nigripalpus \\
\end{tabular} & 28 & & & & & & 28 & 40 & & & & & 40 \\
\hline \begin{tabular}{|l|} 
Cx. (Cux.) quinquefascistus \\
\end{tabular} & & & & 1 & & & 1 & & & & & & \\
\hline Cx. (Cux.) sp. & 13 & & 1 & 1 & 2 & 1 & 18 & 8 & & & & & 8 \\
\hline Cx. (Mel.) bastagarius & & & & & & & & 1 & & & & & 1 \\
\hline CX. (Mel.) contei & & & & 1 & & & 1 & & & & & & \\
\hline CX. (Mel.) dunni & & 1 & & & & & 1 & & & & & & \\
\hline CX. (Mel.) dureti & & & & 1 & & & 1 & & & & & & \\
\hline \begin{tabular}{|l|} 
Cx. (Mel.) grupo Atratus \\
\end{tabular} & & & & & & & & 5 & & & & & 5 \\
\hline \begin{tabular}{|l} 
CX. (Mel.) plectoporpe \\
\end{tabular} & & & & 1 & & & 1 & 2 & & & & & 2 \\
\hline \begin{tabular}{|l|l} 
Cx. (Mel.) rabelloi \\
\end{tabular} & & 5 & & & & & 5 & & & & & & \\
\hline \begin{tabular}{|l|} 
Cx. (Mel.) ribeirensis \\
\end{tabular} & & 2 & 3 & 15 & 21 & & 41 & 52 & 2 & & & & 54 \\
\hline Cx. (Mel.) seção Melanoconion & 1 & 1 & & & & & 2 & 1 & 1 & & & & 2 \\
\hline \begin{tabular}{|l|} 
Ma. amazonensis \\
\end{tabular} & & & 1 & & & & 1 & 2 & & & & 3 & 5 \\
\hline Ma. humeralis & & & & & & & & 1 & & & & & 1 \\
\hline Ma. indubitans & & & & & 1 & & 1 & & & & & & \\
\hline Ma. titillans & 60 & 2 & 3 & 3 & 12 & 3 & 83 & 9 & & 1 & 10 & & 20 \\
\hline Ma. wilsoni & 25 & 7 & 1 & 1 & 4 & 9 & 47 & 20 & 1 & & 6 & 3 & 30 \\
\hline PS. albigenu & & & & 1 & 4 & & 5 & & & & & & \\
\hline \begin{tabular}{|l|l} 
Ps. ciliata \\
\end{tabular} & & & & & & & & 1 & & & & & 1 \\
\hline \begin{tabular}{|l|l} 
Ps. ferox \\
\end{tabular} & & & & 2 & 3 & 1 & 6 & 2 & & 7 & & & 9 \\
\hline Ur. geometrica & & & & & & & & 1 & & & & & 1 \\
\hline \begin{tabular}{|l|} 
Ur. mathesoni \\
\end{tabular} & & 1 & & & & & 1 & $\frac{1}{1}$ & & & & & 1 \\
\hline \begin{tabular}{|l|} 
Ur. nataliae \\
\end{tabular} & & & & & & & & 1 & & & & & 1 \\
\hline Ur. (Ura.) pulcherrima & 2 & & & & & & 2 & 1 & & & & & 1 \\
\hline Wy.sp. & & & & & & & & 1 & & & & & 1 \\
\hline Total & 434 & 776 & 20 & 212 & 159 & 755 & 2356 & 1472 & 15 & 46 & 36 & 29 & 1598 \\
\hline
\end{tabular}


Tabela 23. Número de mosquitos segundo as espécies coletados com armadilha de Shannon no Sítio Caiçara, durante o monitoramento da hidrelétrica de Ourinhos, no período de setembro de 2002 a outubro de 2007.

\begin{tabular}{|c|c|c|c|c|c|c|c|c|c|c|c|c|c|}
\hline \multirow{2}{*}{ Espécie } & \multicolumn{7}{|c|}{ Pré } & \multicolumn{6}{|c|}{ Pós } \\
\hline & $23 / 9 / 2002$ & $21 / 1 / 2003$ & 5/8/2003 & $1 / 12 / 2003$ & \begin{tabular}{|l|}
$29 / 5 / 2004$ \\
\end{tabular} & \begin{tabular}{|l|}
$1 / 12 / 2004$ \\
\end{tabular} & \begin{tabular}{|l|} 
Total \\
\end{tabular} & 13/11/2005 & $3 / 6 / 2006$ & 17/11/2006 & 18/5/2007 & \begin{tabular}{|l|}
$28 / 10 / 2007$ \\
\end{tabular} & Total \\
\hline Ad. squamipennis & 1 & 2 & 1 & & & 1 & 5 & & & & 1 & & \begin{tabular}{|l|l}
1 & \\
\end{tabular} \\
\hline Ae. albopictus & & & & & & 1 & 1 & 1 & & & & & 1 \\
\hline Ae. crinifer & & & & & & & & 2 & & & & & 2 \\
\hline Ae. scapularis & 8 & 1 & 4 & 8 & 1 & 2 & 24 & 16 & 1 & & & & 17 \\
\hline Ae. serratus & & & & & & & & 1 & & & & & 1 \\
\hline An. albitarsis s.l & 6 & & & 1 & & 1 & 8 & & 1 & & & & 1 \\
\hline An. evansae & 2 & & & & & & 2 & & & & & & \\
\hline An. galvaoi & 3 & & & 4 & & & 7 & 1 & & & & & 1 \\
\hline An. strodei & & & & & & & & 1 & & & & & 1 \\
\hline Cq. albicosta & & 1 & & 3 & & & 4 & & & 1 & & & 1 \\
\hline Cq. chrysonotum/albifera & & & & 4 & & & 4 & 1 & & & & & 1 \\
\hline Cq. hermanoi & & & & 11 & & & 11 & 2 & & & & & 2 \\
\hline Cq. juxtamansonia & 123 & 50 & & 203 & 2 & 16 & 394 & 34 & & 11 & 10 & & 55 \\
\hline Cq. nigricans & & 1 & & & & & 1 & & & & & & \\
\hline \begin{tabular}{|l|} 
Cq. shannoni \\
\end{tabular} & 3 & 1 & & 1 & & 3 & 8 & & & & 1 & & 1 \\
\hline \begin{tabular}{|l|} 
Cq. venezuelensis \\
\end{tabular} & 15 & 10 & & 19 & 1 & 2 & 47 & 21 & 1 & & $\frac{1}{6}$ & & 28 \\
\hline Cx. (Cux.) declarator & & 1 & & & & & 1 & & & & & & \\
\hline CX. (Cux.) dolosus & 1 & & & & 1 & & 2 & & & & & & \\
\hline CX. (Cux.) grupo Coronator & 23 & & 23 & 1 & 50 & & 97 & & & & & & \\
\hline CX. (Cux.) nigripalpus & 9 & & & & & & 9 & 1 & & & & & 1 \\
\hline CX. (Cux.) sp. & 14 & 4 & & 6 & & 5 & 29 & $\frac{1}{4}$ & & & & & 4 \\
\hline CX. (Mel.) flabellifer & & & & 1 & & & 1 & & & & & & \\
\hline$C x .($ Mel.) grupo Atratus & & & & 1 & 1 & & 2 & & & & & & \\
\hline CX. (Mel.) ribeirensis & 3 & 4 & 1 & 1 & & 1 & 10 & & & & & & \\
\hline Ma. humeralis & & & & & 1 & & 1 & & & & & & \\
\hline Ma. indubitans & & 3 & & 1 & & & 4 & & & & & & \\
\hline Ma. titillans & & & & 3 & 1 & & 4 & & & 1 & & & 1 \\
\hline Ma. wilsoni & 15 & & & 1 & & & 16 & & & 1 & & & 1 \\
\hline \begin{tabular}{|l|l} 
Ps. ciliata \\
\end{tabular} & & & & 3 & & & 3 & & & & & & \\
\hline Ps. discrucians & & & & 3 & & & 3 & & & & & & \\
\hline Ur. geometrica & & & & & & & & & & & 1 & & 1 \\
\hline Total & 226 & 78 & 29 & 275 & 58 & 32 & 698 & 85 & 3 & 14 & 19 & - & 121 \\
\hline
\end{tabular}


Tabela 24. . Número de mosquitos segundo as espécies coletados com armadilha de Shannon no Córrego Anhumas, durante o monitoramento da hidrelétrica de Ourinhos, no período de setembro de 2002 a outubro de 2007.

\begin{tabular}{|c|c|c|c|c|c|c|c|c|c|c|c|c|c|}
\hline \multirow{2}{*}{ Espécie } & \multicolumn{7}{|c|}{ Pré } & \multicolumn{6}{|c|}{ Pós } \\
\hline & $23 / 9 / 2002$ & $21 / 1 / 2003$ & 5/8/2003 & $1 / 12 / 2003$ & \begin{tabular}{|l|}
$29 / 5 / 2004$ \\
\end{tabular} & $1 / 12 / 2004$ & \begin{tabular}{|l|} 
Total \\
\end{tabular} & \begin{tabular}{|l|}
$13 / 11 / 2005$ \\
\end{tabular} & $3 / 6 / 2006$ & $17 / 11 / 2006$ & $18 / 5 / 2007$ & $\begin{array}{l}28 / 10 / 2007 \\
\end{array}$ & Total \\
\hline \begin{tabular}{|l|} 
Ad. squamippenis \\
\end{tabular} & & 1 & & & & & \begin{tabular}{|l|l|}
1 & \\
\end{tabular} & & & 1 & 7 & 5 & 13 \\
\hline \begin{tabular}{|l|} 
Ae. crinifer \\
\end{tabular} & 3 & & 1 & 1 & & 1 & 6 & 8 & & & & & 8 \\
\hline Ae. scapularis & 4 & 13 & 21 & 45 & 27 & 3 & 113 & 36 & & 1 & 4 & 2 & 43 \\
\hline Ae. serratus & 2 & 1 & 1 & 3 & 32 & 3 & 42 & & & & & & \\
\hline Ae. terrens & & & & & 1 & 1 & 2 & & & & & & \\
\hline An. albitarsis s.l. & & & & & & 1 & 1 & 1 & 6 & & 12 & 9 & 28 \\
\hline \begin{tabular}{|l|} 
An. argyritarsis \\
\end{tabular} & & & 1 & & & & 1 & & & & & & \\
\hline \begin{tabular}{|l} 
An. (Nys.) darlingi \\
\end{tabular} & & & & & & & & & & & & 1 & 1 \\
\hline \begin{tabular}{|l|} 
An. evansae \\
\end{tabular} & & 2 & & 1 & 1 & & 4 & & & & 3 & 3 & 6 \\
\hline \begin{tabular}{|l} 
An. fluminensis \\
\end{tabular} & & & & & & & & & & & & 1 & 1 \\
\hline An. galvaoi & & 1 & & & & & 1 & & & & 7 & 1 & 8 \\
\hline An. strodei & & & & & 1 & & 1 & 1 & 1 & & 1 & 2 & 5 \\
\hline An. triannulatus & & & & & & & & & & & 39 & 4 & 43 \\
\hline \begin{tabular}{|l|} 
Cq. albicosta \\
\end{tabular} & & 10 & & 1 & & & 11 & & & & & & \\
\hline $\begin{array}{l}\text { Cq. chrysonotum/albifera } \\
\end{array}$ & 2 & 45 & & $\frac{1}{2}$ & & & 49 & 1 & & 2 & 2 & & 5 \\
\hline \begin{tabular}{|l|} 
Cq. hermanoi \\
\end{tabular} & & 1 & & & & & 1 & & & & 9 & 5 & 14 \\
\hline \begin{tabular}{|l|} 
Cq. juxtamansonia \\
\end{tabular} & 5 & 32 & & 11 & & 7 & 55 & & & 5 & & 5 & 10 \\
\hline \begin{tabular}{|l|} 
Cq. nigricans \\
\end{tabular} & & & & & & & & & & & 2 & & 2 \\
\hline \begin{tabular}{|l|} 
Cq. shannoni \\
\end{tabular} & & 2 & & & & & 2 & & & & 1 & & 1 \\
\hline Cq. venezuelensis & 10 & 30 & & 5 & 3 & 2 & 50 & 4 & 2 & 9 & 31 & 9 & 55 \\
\hline $\begin{array}{l}\text {. } \\
\text { CX. (Cux.) chidesteri } \\
\end{array}$ & & & & & 1 & 1 & 2 & & 1 & & & 1 & 2 \\
\hline CX. (Cux.) coronator & & & & 1 & 1 & & 2 & & & & & & \\
\hline \begin{tabular}{|l|} 
Cx. (Cux.) declarator \\
\end{tabular} & & & & & 3 & & 3 & & & 1 & & & 1 \\
\hline \begin{tabular}{|l|} 
CX. (Cux.) dolosus \\
\end{tabular} & & & & 3 & 1 & & 4 & & & & & 1 & 1 \\
\hline \begin{tabular}{|l} 
Cx. (Cux.) grupo Coronator \\
\end{tabular} & 22 & & 5 & & 80 & & 107 & & 1 & & & & 1 \\
\hline \begin{tabular}{|l|} 
Cx. (Cux.) nigripalpus \\
\end{tabular} & 3 & & & & & & 3 & & & & & & \\
\hline Cx. (Cux.) sp. & 9 & 1 & 4 & 1 & 12 & 2 & 29 & & & & 7 & & 7 \\
\hline \begin{tabular}{|l} 
CX. (Mel.) aureonotatus \\
\end{tabular} & & & & & & & & & & 3 & 1 & 5 & 9 \\
\hline \begin{tabular}{|l|} 
CX. (Mel.) bastagarius \\
\end{tabular} & & & & & & & & 1 & & & & & 1 \\
\hline Cx. (Mel.) dureti & & & & & & & & & & & & 3 & 3 \\
\hline \begin{tabular}{|l|} 
Cx. (Mel.) idottus \\
\end{tabular} & & & & & & & & & & & & 1 & 1 \\
\hline \begin{tabular}{|l|} 
Cx. (Mel.) ocossa \\
\end{tabular} & & & & & & & & & & & & 4 & 4 \\
\hline \begin{tabular}{|l|} 
Cx. (Mel.) pilosus \\
\end{tabular} & & & & & & & & & & & & 1 & 1 \\
\hline CX. (Mel.) plectoporpe & & & & & & & & 1 & & & & & 1 \\
\hline Cx. (Mel.) ribeirensis & 1 & 10 & & & & 1 & 12 & 1 & & & & & 1 \\
\hline \begin{tabular}{|l} 
CX. (Mel.) seção Melanoconion \\
\end{tabular} & & 1 & & & & 1 & 2 & & & & 4 & 19 & 23 \\
\hline CX. (Mel.) vaxus & & & & & & 1 & 1 & 3 & 1 & & & 2 & 6 \\
\hline Hg. leucocelaenus & & & & & & 1 & 1 & & & & & & \\
\hline \begin{tabular}{|l|} 
Ma. indubitans \\
\end{tabular} & & & & 1 & & & 1 & & & & & 1 & 1 \\
\hline Ma. titillans & 2 & 5 & 1 & & & 1 & 9 & & & & 5 & 8 & 13 \\
\hline Ma. wilsoni & 22 & 3 & & 2 & & 1 & 28 & 1 & & 6 & 17 & 12 & 36 \\
\hline \begin{tabular}{|l} 
Ps. albigenu \\
\end{tabular} & & & & 2 & & & 2 & & & & & & \\
\hline \begin{tabular}{|l|} 
Ps. albipes \\
\end{tabular} & & & & 2 & & & 2 & & & & & & \\
\hline \begin{tabular}{|l|} 
Ps. ciliata \\
\end{tabular} & & & & 1 & & & 1 & & & & & & \\
\hline \begin{tabular}{|l|} 
Ps. discrucians \\
\end{tabular} & & 1 & & & & & 1 & 2 & & & & & 2 \\
\hline \begin{tabular}{|l} 
Ps. ferox \\
\end{tabular} & & & & 8 & & 2 & 10 & 2 & & & & & 2 \\
\hline Ur. geometrica & & 1 & & & & 1 & 2 & 2 & & & & & 2 \\
\hline Total & 85 & 160 & 34 & 90 & 163 & 30 & 562 & 64 & 12 & 28 & 152 & 105 & 361 \\
\hline
\end{tabular}


Tabela 25. Número de mosquitos segundo as espécies coletados no Canteiro de Obras, durante o monitoramento da hidrelétrica de Ourinhos, no período de setembro de 2002 a outubro de 2007

\begin{tabular}{|c|c|c|c|c|c|c|c|c|c|c|c|c|c|}
\hline \multirow{2}{*}{ Espécie } & \multicolumn{7}{|c|}{ Pré } & \multicolumn{6}{|c|}{ Pós } \\
\hline & $23 / 9 / 2002$ & 21/1/2003 & $5 / 8 / 2003$ & \begin{tabular}{|l|}
$1 / 12 / 2003$ \\
\end{tabular} & \begin{tabular}{|l|}
$29 / 5 / 2004$ \\
\end{tabular} & 1/12/2004 & Total & 13/11/2005 & \begin{tabular}{|l|}
$3 / 6 / 2006$ \\
\end{tabular} & \begin{tabular}{|l|}
$17 / 11 / 2006$ \\
\end{tabular} & \begin{tabular}{|l|l|}
$18 / 5 / 2007$ \\
\end{tabular} & $28 / 10 / 2007$ & Total \\
\hline \begin{tabular}{|l} 
Ad. squamippenis \\
\end{tabular} & 6 & 4 & & 10 & 4 & 2 & 26 & 4 & 3 & & & 2 & 9 \\
\hline \begin{tabular}{|l|} 
Ae. albopictus \\
\end{tabular} & & 2 & & & & & 2 & 3 & & & & & 3 \\
\hline Ae. crinifer & & & & 1 & & & 1 & & & & & & \\
\hline Ae. fluviatilis & & & & 1 & 8 & 14 & 23 & 75 & & & & & 75 \\
\hline Ae. scapularis & 21 & 2 & & 6 & 136 & & 165 & 23 & & 3 & 2 & 3 & 31 \\
\hline \begin{tabular}{|l|} 
Ae. serratus \\
\end{tabular} & & & & & 1 & 1 & 2 & 1 & & & & & 1 \\
\hline An. (Ano.) maculipes & & & & & & & & $\frac{1}{1}$ & & & & & 1 \\
\hline An. (Nys.) albitarsis & & & 4 & & & & 4 & & 3 & & 2 & & 5 \\
\hline An. (Nys.) albitarsis s.l & 6 & 1 & 8 & & & & 15 & & & & & & \\
\hline An. (Nys.) galvaoi & 52 & 20 & 3 & & 3 & 2 & 80 & & 1 & & & & 1 \\
\hline An. (Nys.) sp & & 5 & & & & & 5 & & & & 1 & & 1 \\
\hline An. (Nys.) strodei/ rondoni & & 3 & 1 & & & & 4 & & & & & & \\
\hline \begin{tabular}{|l|} 
An. argyritarsis \\
\end{tabular} & 13 & 6 & 1 & & 1 & & 21 & & 4 & & 2 & & 6 \\
\hline \begin{tabular}{|l|} 
An. evansae \\
\end{tabular} & & & & & 1 & 1 & 2 & & & & & & \\
\hline An. lutzi & & & & & & & & 1 & & & & & 1 \\
\hline An. strodei & & & & & & & & 2 & & & & & 2 \\
\hline An. triannulatus & 28 & 1 & & 6 & 14 & & 49 & & & & & & \\
\hline Cq. albicosta & & 512 & & 8 & 3 & 4 & 527 & 64 & & & & & 64 \\
\hline Cq. chrysonotum & 39 & 24 & & & & 57 & 120 & 2 & & 6 & & 5 & 13 \\
\hline Cq. chrysonotum/albifera & 52 & 69 & & 42 & 6 & 628 & 797 & 611 & & 30 & 6 & 6 & 653 \\
\hline Cq. hermanoi & 1 & 1 & & 35 & 8 & 6 & 51 & 148 & & & 10 & 5 & 163 \\
\hline \begin{tabular}{|l|} 
Cq. juxtamansonia \\
\end{tabular} & 55 & 3 & 1 & 11 & 2 & 32 & 104 & 40 & & 3 & 1 & 2 & 46 \\
\hline \begin{tabular}{|l|} 
Cq. nigricans \\
\end{tabular} & & $\frac{3}{2}$ & & 52 & & & 54 & 63 & & & & 3 & 66 \\
\hline Cq. shannoni & 2 & 4 & & & 2 & 2 & 10 & & & & & & \\
\hline Cq. venezuelensis & 71 & 21 & & 11 & 12 & 9 & 124 & 359 & 1 & & 1 & & 361 \\
\hline CX. (Ads.) amazonenzis & & 17 & & 3 & 4 & & 24 & 7 & & & & & 7 \\
\hline CX. (Cux.) bidens & & & & & & & & & & 1 & & & 1 \\
\hline CX. (Cux.) chidesteri & & & 1 & & & & 1 & & & & & & \\
\hline CX. (Cux.) coronator & 3 & 5 & & & 12 & & 20 & & 22 & 3 & 7 & 4 & 36 \\
\hline CX. (Cux.) declarator & 1 & & & 4 & & & 5 & 1 & 1 & & 1 & & 3 \\
\hline Cx. (Cux.) dolosus & & & 1 & 1 & 1 & & 3 & $\frac{1}{3}$ & & 3 & $\frac{1}{1}$ & 3 & 10 \\
\hline CX. (Cux.) dolosus/eduardoi & & & & & & 2 & 2 & & 4 & & & & 4 \\
\hline \begin{tabular}{|l} 
CX. (Cux.) grupo Coronator \\
\end{tabular} & 31 & 28 & & & 24 & & 83 & & 62 & 36 & 16 & 9 & 123 \\
\hline Cx. (Cux.) mollis & & & & 2 & & & 2 & & & & & & \\
\hline CX. (Cux.) nigripalpus & 29 & & & & & & 29 & 40 & & & & & 40 \\
\hline Cx. (Cux.) quinquefascistus & & & & 1 & & 10 & 11 & 39 & & & & & 39 \\
\hline Cx. (Cux.) sp. & 15 & 12 & 1 & 13 & 2 & 3 & 46 & 8 & & 7 & 3 & 3 & 21 \\
\hline$C X .(M C X$.$) imitator$ & & & & & & & & & & 10 & & & 10 \\
\hline CX. (Mel.) bastagarius & & & & & & & & 1 & & & & & 1 \\
\hline CX. (Mel.) contei & & & & 1 & & & 1 & & & & & & \\
\hline CX. (Mel.) dunni & & 1 & & & & & 1 & & & & & & \\
\hline CX. (Mel.) dureti & & & & 1 & & & 1 & & & & & & \\
\hline CX. (Mel.) grupo Atratus & & & & & & & & 5 & & & 1 & & 6 \\
\hline Cx. (Mel.) ocossa & & & & & 1 & & 1 & & & & & & \\
\hline CX. (Mel.) plectoporpe & & 1 & & 1 & & & 2 & 2 & & & & & 2 \\
\hline CX.(Mel.) rabelloi & & 5 & & & & & 5 & 1 & & & & & 1 \\
\hline Cx. (Mel.) ribeirensis & 2 & $\frac{5}{2}$ & 5 & 15 & 22 & & 46 & 52 & 2 & & & & 54 \\
\hline Cx. (Mel.) seção Melanoconion & 1 & 3 & & & 1 & & 5 & 1 & 2 & & & & 3 \\
\hline CX. (Mel.) sphatulatus & & & & & & & & 1 & & & & & 1 \\
\hline Ma. amazonensis & & & 1 & & & & 1 & 2 & & & & 3 & 5 \\
\hline Ma. humeralis & & & & & & & & 1 & & & & & $\frac{5}{1}$ \\
\hline Ma. indubitans & & & & & 1 & & 1 & & & & & & \\
\hline Ma. titillans & 60 & 2 & 3 & 3 & 12 & 3 & 83 & 9 & & 1 & 10 & & 20 \\
\hline Ma. wilsoni & 25 & 7 & 1 & 1 & 4 & 9 & 47 & 20 & 1 & & 6 & 3 & 30 \\
\hline Ps. albigenu & & & & $\frac{1}{2}$ & $\frac{4}{5}$ & & $\frac{41}{7}$ & & & & & & \\
\hline Ps. ciliata & & & & & & 5 & 5 & 1 & & & & & 1 \\
\hline Ps. confinnis & & & & & 5 & & 5 & & & & & & \\
\hline Ps. ferox & 1 & & & 2 & 3 & 1 & 7 & 2 & & 8 & & & 10 \\
\hline Ps. lutzii & & & & & & 1 & 1 & & & & & & \\
\hline Sa.sp. & & & & & & & & & & 1 & & & 1 \\
\hline Ur. (Ura.) lowii & & & & & & & & & 1 & 1 & & & 2 \\
\hline Ur. (Ura.) pulcherrima & 3 & & & & & 2 & 5 & 1 & & 2 & 1 & & 4 \\
\hline Ur. geometrica & & & 3 & 2 & & & 5 & $\frac{1}{10}$ & & 3 & & 11 & $\frac{4}{24}$ \\
\hline Ur. mathesoni & & 2 & 1 & & 1 & 2 & 6 & 1 & & & 3 & 1 & 5 \\
\hline Ur. nataliae & & & 2 & 1 & & & 3 & 1 & & & & 1 & 2 \\
\hline Wy. rooti & & & & & & & & & & 16 & & & 16 \\
\hline Wy. sp. & 1 & 3 & & 1 & & & 5 & 1 & & & 9 & & 10 \\
\hline Total & 518 & 768 & 37 & 237 & 299 & 796 & 2655 & 1607 & 107 & 134 & 83 & 64 & 1995 \\
\hline
\end{tabular}


Tabela 26. . Número de mosquitos segundo as espécies coletados no Sítio Caiçara, durante o monitoramento da hidrelétrica de Ourinhos, no período de setembro de 2002 a outubro de 2007

\begin{tabular}{|c|c|c|c|c|c|c|c|c|c|c|c|c|c|}
\hline \multirow{2}{*}{ Espécie } & \multicolumn{7}{|c|}{ Pré } & \multicolumn{6}{|c|}{$\begin{array}{l}\text { Pós } \\
\end{array}$} \\
\hline & $23 / 9 / 2002$ & 21/1/2003 & $5 / 8 / 2003$ & $1 / 12 / 2003$ & $29 / 5 / 2004$ & 1/12/2004 & Total & 13/11/2005 & 3/6/2006 & \begin{tabular}{|l|}
$17 / 11 / 2006$ \\
\end{tabular} & \begin{tabular}{|l|}
$18 / 5 / 2007$ \\
\end{tabular} & $28 / 10 / 2007$ & Total \\
\hline \begin{tabular}{|l} 
Ad. squamipennis \\
\end{tabular} & 1 & 2 & 1 & & & 1 & 5 & & 1 & \begin{tabular}{|l|}
4 \\
\end{tabular} & \begin{tabular}{|l|}
63 \\
\end{tabular} & 1 & 69 \\
\hline \begin{tabular}{|l|} 
Ae. albopictus \\
\end{tabular} & & 2 & 3 & 3 & 1 & 11 & 20 & 1 & & & & & 1 \\
\hline \begin{tabular}{|l|} 
Ae. crinifer \\
\end{tabular} & & & & & & & & 10 & & & & & 10 \\
\hline Ae. fluviatilis & & & & & & & & & & & & 22 & 22 \\
\hline \begin{tabular}{|l|} 
Ae. nubilus \\
\end{tabular} & & & & & & & & 1 & & & & & 1 \\
\hline \begin{tabular}{|l|} 
Ae. scapularis \\
\end{tabular} & 36 & 9 & 4 & 11 & 11 & 3 & 74 & 23 & 1 & & & & 24 \\
\hline Ae. serratus & & & & & & & & 2 & & & & & $\frac{2}{2}$ \\
\hline Ae. serratus/nubilus & & & & & & & & 3 & & & & & 3 \\
\hline \begin{tabular}{|l|} 
Ae. terrens \\
\end{tabular} & 15 & 2 & & & & & 17 & & & & & & \\
\hline \begin{tabular}{|l|} 
An. (Nys.) albitarsis \\
\end{tabular} & & & & 2 & & & 2 & & 9 & 3 & 18 & 4 & 34 \\
\hline An. (Nys.) darlingi & & & & & & & & & & & 4 & & 4 \\
\hline An. (Nys.) sp & & & & & & & & & & 1 & & 5 & 6 \\
\hline An. (Nys.) strodei & & & & & & & & 1 & 3 & & 4 & & 8 \\
\hline \begin{tabular}{|l} 
An. (Nys.) strodei/ rondoni \\
\end{tabular} & 2 & & 5 & & & & 7 & & & & & & \\
\hline \begin{tabular}{|l|} 
An. (Nys.) triannulatus \\
\end{tabular} & & & & & & & & & & & 11 & & 11 \\
\hline \begin{tabular}{|l|l} 
An. albitarsis s.l \\
\end{tabular} & 6 & & & 1 & & 1 & 8 & & 1 & & & & 1 \\
\hline An. argyritarsis & & 1 & & & & & 1 & & 2 & & 5 & 5 & 12 \\
\hline \begin{tabular}{|l|} 
An. evansae \\
\end{tabular} & 2 & & & & & & 2 & & & & & & \\
\hline \begin{tabular}{|l|} 
An. galvaoi \\
\end{tabular} & 3 & & & 4 & & & 7 & 1 & & & & & 1 \\
\hline Cq. albicosta & & 1 & & 3 & & & 4 & & & 1 & & & 1 \\
\hline \begin{tabular}{|l|} 
Cq. chrysonotum/albifera \\
\end{tabular} & & & & 4 & & & 4 & 1 & & & & & 1 \\
\hline \begin{tabular}{|l|} 
Cq. hermanoi \\
\end{tabular} & & & & 11 & & & 11 & 2 & & & & & 2 \\
\hline Cq. juxtamansonia & 123 & 50 & & 203 & 2 & 16 & 394 & 35 & & 11 & 10 & & 56 \\
\hline \begin{tabular}{|l|} 
Cq. nigricans \\
\end{tabular} & & 1 & & & & & 1 & & & & & & \\
\hline Cq. shannoni & 3 & 1 & & 1 & & 3 & 8 & & & & 1 & & 1 \\
\hline \begin{tabular}{|l|} 
Cq. venezuelensis \\
\end{tabular} & 15 & 12 & & 19 & 1 & 2 & 49 & 22 & 2 & & 8 & 2 & 34 \\
\hline \begin{tabular}{|l|} 
Cx. $($ Cux. $)$ chidesteri \\
\end{tabular} & 3 & & & 1 & & & 4 & & & 11 & & 4 & 15 \\
\hline CX. (Cux.) coronator & & 9 & 4 & 1 & 16 & 17 & 47 & 3 & & 1 & 4 & 1 & 9 \\
\hline \begin{tabular}{|l|} 
Cx. $($ Cux. $)$ declarator \\
\end{tabular} & 2 & 10 & 6 & 1 & 7 & 1 & 27 & & & & & & \\
\hline \begin{tabular}{|l|l} 
Cx. (Cux.) dolosus \\
\end{tabular} & 3 & 3 & 31 & 1 & 35 & & 73 & 5 & & & & & 5 \\
\hline Cx. (Cux.) dolosus/eduardoi & & & & & 5 & 2 & 7 & & 1 & & & & 1 \\
\hline \begin{tabular}{|l|} 
Cx. (Cux.) eduardoi \\
\end{tabular} & & & & & 2 & & 2 & & & & & & \\
\hline \begin{tabular}{|l} 
Cx. (Cux.) grupo Coronator \\
\end{tabular} & 23 & 12 & 23 & 22 & 91 & 34 & 205 & & 3 & & 12 & 1 & 16 \\
\hline \begin{tabular}{|l|} 
CX. (Cux.) lygrus \\
\end{tabular} & & 1 & & 1 & 1 & & 3 & 1 & & & & & 1 \\
\hline \begin{tabular}{|l|} 
CX. (Cux.) mollis \\
\end{tabular} & & & 5 & 2 & & & 7 & & 1 & 5 & & & 6 \\
\hline \begin{tabular}{|l|} 
Cx. (Cux.) nigripalpus \\
\end{tabular} & 9 & & & & & & 9 & 1 & & & & & 1 \\
\hline CX. (Cux.) quinquefascistus & & & 2 & & & & 2 & & & & & & \\
\hline $\begin{array}{ll}\text { CX. (Cux.) sp. } \\
\end{array}$ & 22 & 6 & & 19 & 6 & 7 & 60 & 14 & & 1 & 1 & 9 & 25 \\
\hline \begin{tabular}{|l|} 
CX. (Mel.) aureonotatus \\
\end{tabular} & & & & & & & & & & 3 & & & 3 \\
\hline \begin{tabular}{|l|} 
CX. (Mel.) dureti \\
\end{tabular} & & & & & & & & & & & 1 & & 1 \\
\hline CX. (Mel.) flabellifer & & & & 1 & & & 1 & & & & & & \\
\hline \begin{tabular}{|l} 
CX. (Mel.) grupo Atratus \\
\end{tabular} & & & & 1 & 1 & & 2 & & & & & & \\
\hline \begin{tabular}{|l|} 
Cx. (Mel.) ocossa \\
\end{tabular} & & & & & 1 & & 1 & & & & & & \\
\hline \begin{tabular}{|l|} 
CX. (Mel.) pilosus \\
\end{tabular} & 1 & & & & & & 1 & & & & & & \\
\hline \begin{tabular}{|l|} 
Cx. (Mel.) ribeirensis \\
\end{tabular} & 3 & 4 & 1 & 1 & & 1 & 10 & & & & & & \\
\hline CX. (Mel.) seção Melanoconion & & & & & & & & & & & 2 & & 2 \\
\hline CX. (Mel.) sp & & & & & & & & & 4 & & & & 4 \\
\hline CX. (Mel.) vaxus & & & & & & & & & 1 & & 1 & & 2 \\
\hline Ma. humeralis & & & & & 1 & & 1 & & & & & & \\
\hline \begin{tabular}{|l|} 
Ma. indubitans \\
\end{tabular} & & 3 & & 1 & & & 4 & & & & & & \\
\hline Ma. titillans & & & & 3 & 1 & & 4 & & & 1 & & & 1 \\
\hline Ma. wilsoni & 15 & & & 1 & & & 16 & & & 1 & & & 1 \\
\hline \begin{tabular}{|l|l} 
Ps. ciliata \\
\end{tabular} & & & & 3 & & & 3 & & & & & & \\
\hline \begin{tabular}{|l|} 
Ps. discrucians \\
\end{tabular} & & & & 3 & & & 3 & & & & & & \\
\hline Ur. (Ura.) lowii & & & & & & & & & & 3 & & & 3 \\
\hline Ur. (Ura.) pulcherrima & & 1 & & & & & 1 & & & & & & \\
\hline \begin{tabular}{|l|} 
Ur. (Ura.) sp. \\
\end{tabular} & & & & & & & & & & & 1 & & 1 \\
\hline \begin{tabular}{|l|} 
Ur. geometrica \\
\end{tabular} & & & & & & & & & & & 1 & & 1 \\
\hline \begin{tabular}{|l|} 
Wy. sp. \\
\end{tabular} & & & 1 & & & & 1 & 3 & & & & & 3 \\
\hline Total & 287 & 130 & 86 & 324 & 182 & 99 & 1108 & 129 & 29 & 46 & 147 & 54 & 405 \\
\hline
\end{tabular}


Tabela 27. Número de mosquitos segundo as espécies coletados no Córrego Anhumas, durante o monitoramento da hidrelétrica de Ourinhos, no período de setembro de 2002 a outubro de 2007.

\begin{tabular}{|c|c|c|c|c|c|c|c|c|c|c|c|c|c|}
\hline \multirow{2}{*}{ Espécie } & \multicolumn{7}{|c|}{ Pré } & \multicolumn{6}{|c|}{ Pós } \\
\hline & $23 / 9 / 2002$ & $\begin{array}{l}21 / 1 / 2003 \\
\end{array}$ & $5 / 8 / 2003$ & 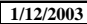 & 29/5/2004 & 1/12/2004 & Total & 13/11/2005 & \begin{tabular}{|l|}
$3 / 6 / 2006$ \\
\end{tabular} & 17/11/2006 & 18/5/2007 & $28 / 10 / 2007$ & Total \\
\hline \begin{tabular}{|l|} 
Ad. squamipennis \\
\end{tabular} & 12 & 4 & 24 & & & & 40 & 32 & 26 & 12 & 24 & 14 & 108 \\
\hline Ae. crinifer & $\frac{12}{3}$ & & $\frac{24}{2}$ & 1 & & 1 & $\frac{40}{7}$ & 11 & & & & & 11 \\
\hline Ae. nubilus & & & & & 5 & & 5 & & & & & & \\
\hline Ae. scapularis & 6 & 20 & 23 & 65 & 67 & 4 & 185 & 38 & 1 & 1 & 4 & 2 & 46 \\
\hline \begin{tabular}{|l|} 
Ae. serratus \\
\end{tabular} & 2 & 2 & 1 & 3 & 37 & 3 & 48 & & & & & & \\
\hline \begin{tabular}{|l|} 
Ae. terrens \\
\end{tabular} & & & & & 1 & 1 & 2 & & & & & & \\
\hline An. (Nys.) albitarsis & & & 1 & & $\frac{1}{1}$ & 8 & 10 & 1 & 16 & 14 & 7 & 26 & 64 \\
\hline \begin{tabular}{|l} 
An. (Nys.) darlingi \\
\end{tabular} & & & & & & & & & & & 2 & 1 & 3 \\
\hline An. (Nys.) galvaoi & & 3 & & & 1 & & 4 & & & & 7 & 1 & \\
\hline \begin{tabular}{|l|} 
An. (Nys.) sp \\
\end{tabular} & & 2 & & 1 & & & 3 & & & & & 2 & 2 \\
\hline An. (Nys.) strodei & & & & & 1 & & 1 & 1 & 2 & & 2 & 2 & 7 \\
\hline \begin{tabular}{|l|} 
An. albitarsis s.l \\
\end{tabular} & & 1 & & & & 1 & 2 & 1 & 6 & 1 & 12 & 9 & 29 \\
\hline An. argyritarsis & 9 & 19 & 1 & & 15 & & 44 & & 1 & & & 1 & 2 \\
\hline \begin{tabular}{|l|} 
An. evansae \\
\end{tabular} & & 2 & & 1 & 1 & & 4 & & & & 4 & 3 & 7 \\
\hline An. fluminensis & & & & & & & & & & & & 1 & 1 \\
\hline An. triannulatus & & & & & & & & & & & 40 & 4 & 44 \\
\hline Cq. albicosta & & 10 & & 1 & & & 11 & & & & & & \\
\hline \begin{tabular}{|l} 
Cq. chrysonotum/albifera \\
\end{tabular} & 2 & 45 & & 2 & & & 49 & 1 & & 2 & 2 & & 5 \\
\hline Cq. hermanoi & & 1 & & & & & 1 & & & & 9 & 5 & 14 \\
\hline Cq. juxtamansonia & 5 & 32 & & 12 & & 7 & 56 & & & 5 & & 5 & 10 \\
\hline \begin{tabular}{|l|} 
Cq. nigricans \\
\end{tabular} & & & & & & & & & & & 2 & & 2 \\
\hline \begin{tabular}{|l|} 
Cq. shannoni \\
\end{tabular} & & 2 & & & & & 2 & & & & 1 & & 1 \\
\hline \begin{tabular}{|l|} 
Cq. venezuelensis \\
\end{tabular} & 10 & 37 & & 5 & 3 & 2 & 57 & 4 & 2 & 9 & 32 & 9 & 56 \\
\hline CX. (Cux.) bidens & 1 & & & & & & 1 & & & 3 & & 1 & 4 \\
\hline $\begin{array}{l}\text { Cx. }(\text { Cux.) chidesteri } \\
\end{array}$ & 2 & 3 & 7 & & 5 & 1 & 18 & & 1 & 1 & & 1 & 3 \\
\hline \begin{tabular}{|l|} 
Cx. (Cux.) coronator \\
\end{tabular} & 6 & 5 & & 9 & 6 & 1 & 27 & & 2 & & & 2 & 4 \\
\hline \begin{tabular}{|l} 
Cx. $($ Cux. $)$ declarator \\
\end{tabular} & 2 & 7 & & 2 & 17 & 3 & 31 & 2 & & 2 & & 1 & 5 \\
\hline \begin{tabular}{|l} 
CX. (Cux.) dolosus \\
\end{tabular} & 1 & 11 & 1 & 3 & 4 & & 20 & & & & & 2 & 2 \\
\hline CX. (Cux.) grupo Coronator & 27 & 7 & 5 & 6 & 96 & & 141 & & 32 & 2 & & 9 & 43 \\
\hline \begin{tabular}{|l|} 
Cx. (Cux.) lygrus \\
\end{tabular} & & & & & 1 & & 1 & & & & & & \\
\hline \begin{tabular}{|l|} 
Cx. (Cux.) mollis \\
\end{tabular} & & & & 1 & & & 1 & & & & & & \\
\hline \begin{tabular}{|l|} 
Cx. (Cux.) nigripalpus \\
\end{tabular} & 3 & 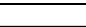 & & & & & 3 & & & & & & \\
\hline \begin{tabular}{|l} 
Cx. (Cux.) quinquefascistus \\
\end{tabular} & & & & & & & & & & & 1 & & 1 \\
\hline \begin{tabular}{|l|} 
Cx. (Cux.) sp. \\
\end{tabular} & 13 & 1 & 4 & 5 & 13 & 2 & 38 & 4 & 2 & 2 & 11 & 10 & 29 \\
\hline \begin{tabular}{|l|}
$C x .(M c x$.$) imitator$ \\
\end{tabular} & & & & 5 & & & 5 & & & & & & \\
\hline$C x$.(Mel.) aureonotatus & & & & & & & & & & 6 & 1 & 5 & 12 \\
\hline \begin{tabular}{|l|} 
Cx. (Mel.) bastagarius \\
\end{tabular} & & & & & & 1 & 1 & 1 & & & & & 1 \\
\hline \begin{tabular}{|l|} 
CX. (Mel.) dureti \\
\end{tabular} & & & & & & & & & & & & 3 & 3 \\
\hline \begin{tabular}{|l}
$C x$. (Mel.) grupo Atratus \\
\end{tabular} & & 1 & & & & & 1 & & & & & & \\
\hline \begin{tabular}{|l|} 
Cx. (Mel.) idottus \\
\end{tabular} & & & & & & & & & & & & 1 & 1 \\
\hline CX. (Mel.) ocossa & & & & & & & & & & & 1 & 4 & 5 \\
\hline \begin{tabular}{|l|} 
Cx. (Mel.) pilosus \\
\end{tabular} & & & & & & & & & & & & 1 & 1 \\
\hline Cx. (Mel.) plectoporpe & & & & & & & & 1 & & & & & 1 \\
\hline Cx. (Mel.) ribeirensis & 1 & 14 & & & 1 & 1 & 17 & 1 & & & & & 1 \\
\hline CX. (Mel.) seção Melanoconion & & 2 & & & & 1 & 3 & & 3 & 1 & 4 & 19 & 27 \\
\hline \begin{tabular}{|l|} 
Cx. (Mel.) sp. \\
\end{tabular} & & 2 & & & & & 2 & & & & & & \\
\hline Cx. (Mel.) vaxus & & & & & & 3 & 3 & 3 & 1 & & & 4 & 8 \\
\hline Cx. (Mel.) zeteki & & & & & & & & & & & 1 & & 1 \\
\hline CX. saltanensis & & 1 & & & 1 & & 2 & & & & & & \\
\hline Hg. leucocelaenus & & & & & & 1 & 1 & & & & & & \\
\hline \begin{tabular}{|l|} 
Li. durhami \\
\end{tabular} & & & & 1 & & & 1 & & & & & & \\
\hline \begin{tabular}{|l|l|l} 
Ma. flaveola \\
\end{tabular} & & & & & & & & & & & & 3 & 3 \\
\hline Ma. indubitans & & 1 & & 1 & & & 2 & & & & & 1 & 1 \\
\hline Ma. titillans & 2 & 5 & 1 & & & 1 & 9 & & & & 5 & 9 & 14 \\
\hline Ma. wilsoni & 22 & 3 & & 2 & & 1 & 28 & 1 & & 6 & 17 & 13 & 37 \\
\hline Ps. albigenu & & & & 2 & & & 2 & & & & & & \\
\hline \begin{tabular}{|l|} 
Ps. albipes \\
\end{tabular} & & & & 2 & & & 2 & & & & & & \\
\hline \begin{tabular}{|l|} 
Ps. ciliata \\
\end{tabular} & & & 1 & 1 & & & 2 & & & & & & \\
\hline \begin{tabular}{|l} 
Ps. cingulata \\
\end{tabular} & & & & 2 & & & 2 & & & & & & \\
\hline \begin{tabular}{|l} 
Ps. confinnis \\
\end{tabular} & & & & & 1 & & 1 & & & & & & \\
\hline \begin{tabular}{|l} 
Ps. discrucians \\
\end{tabular} & & 1 & & & & & 1 & 2 & & & & & 2 \\
\hline \begin{tabular}{|l|} 
Ps. ferox \\
\end{tabular} & & & & 8 & 1 & 2 & 11 & 3 & & & & & 3 \\
\hline \begin{tabular}{|l|} 
Ps. sp. \\
\end{tabular} & 2 & & & & & & 2 & & & & & & \\
\hline Ur. (Ura.) lowii & & & & & 1 & 1 & 2 & & & & & & \\
\hline Ur. (Ura.) pulcherrima & 1 & & 2 & & & & 3 & 6 & & & & & 6 \\
\hline Ur. geometrica & & 2 & 1 & & & 1 & 4 & 2 & & & & & 2 \\
\hline Ur. sp & & & & & & & & & & & & 1 & 1 \\
\hline \begin{tabular}{|l|} 
Wy. medioalbipes \\
\end{tabular} & & & & 24 & & & 24 & & & & & & \\
\hline Total & 132 & 246 & 74 & 165 & 279 & 47 & 943 & 115 & 95 & 67 & 189 & 175 & 633 \\
\hline
\end{tabular}


Anexo V-Lattes

\section{Renato Sinnhofer Sugimoto}

Bolsista de Mestrado do CNPq

possui graduação em Ciências Biológicas - Bacharelado e Licenciatura pela Universidade de São Paulo Tem experiência na área de Saúde Coletiva, com ênfase em Entomologia Médica, e na área de Ecologia, com ênfase em Ecologia Comportamental e Dinâmica Populacional.

(Texto informado pelo autor)

Última atualização do currículo em 30/04/2008

Endereço para acessar este CV:

Certificado

http://lattes.cnpq.br/8868970424844776

\section{pelo autor em}

30/04/08

\section{Delsio Natal}

Possui graduação em Ciências Biológicas pela Universidade de São Paulo (1977), mestrado em Saúde Pública pela Universidade de São Paulo (1982) e doutorado em Saúde Pública pela Universidade de São Paulo (1986). Atualmente é professor associado da Universidade de São Paulo. Tem experiência na área de Saúde Coletiva, com ênfase em Epidemiologia, atuando principalmente nos seguintes temas: culicidae, aedes aegypti, dengue, culex quinquefasciatus e controle.

(Texto informado pelo autor)

Última atualização do currículo em 21/05/2009

Endereço para acessar este CV:

http://lattes.cnpq.br/2025787312261734
Certificado

pelo autor em

21/05/09 